\title{
Konzeption und Entwicklung eines Probandenmanagementsystems am Beispiel der Universitätsmedizin Göttingen
}

\author{
Dissertation \\ zur Erlangung des mathematisch-naturwissenschaftlichen Doktorgrades \\ „Doctor rerum naturalium“ \\ der Georg-August-Universität Göttingen \\ im Promotionsprogramm in Computer Science (PCS) \\ der Georg-August University School of Science (GAUSS)
}

vorgelegt von

Jens Schwanke

aus Heidelberg

Göttingen, 2015 


\section{Betreuungsausschuss:}

Prof. Dr. Otto Rienhoff, Institut für Medizinische Informatik, Universitätsmedizin Göttingen Prof. Dr. Ramin Yahyapour, Institut für Informatik, Universität Göttingen

Prof. Dr. Dieter Hogrefe, Institut für Informatik, Universität Göttingen

Prof. Dr. Ulrich Sax, Institut für Medizinische Informatik, Universitätsmedizin Göttingen

\section{Mitglieder der Prüfungskommission:}

Referent:

Prof. Dr. Otto Rienhoff, Institut für Medizinische Informatik, Universitätsmedizin Göttingen

Korreferent:

Prof. Dr. Ramin Yahyapour, Institut für Informatik, Universität Göttingen

Weitere Mitglieder der Prüfungskommission:

Prof. Dr. Rolf Bendl, Fakultät für Informatik, Hochschule Heilbronn

Prof. Dr. Tim Friede, Institut für Medizinische Statistik, Universitätsmedizin Göttingen

Prof. Dr. Dieter Hogrefe, Institut für Informatik, Universität Göttingen

Prof. Dr. Ulrich Sax, Institut für Medizinische Informatik, Universitätsmedizin Göttingen

Tag der mündlichen Prüfung: 01.12.2015 


\section{Zusammenfassung}

In medizinischen Forschungsprojekten besteht ein großer Bedarf an effizienter und einheitlicher Verwaltung sowie langfristiger Nachverfolgung von Probanden. Dieser Bedarf entsteht in Deutschland unter anderem aus der enger werdenden Verbindung von Versorgung und Forschung, der zunehmenden Vernetzung der medizinischen Forschung, sowie der Etablierung neuer Einrichtungen, wie beispielsweise Biobanken an Universitätskliniken.

Im Rahmen dieser Dissertation wurde am Beispiel der Universitätsmedizin Göttingen untersucht, ob die Einführung eines Probandenmanagementsystems den Bedarf von Forschern an Effizienz, Einheitlichkeit und Langfristigkeit erfüllt. Im ersten Schritt wurde eine Anforderungsanalyse durchgeführt. Grundlage dieser Analyse bildeten 17 Interviews mit Stakeholdern der Universitätsmedizin Göttingen. Darunter befanden sich Ärzte, wissenschaftliche Mitarbeiter, Studienassistenten sowie IT-Verantwortliche. Anhand der Interviews wurden 16 Ziele an ein Probandenmanagementsystem formuliert. Auf Basis dieser Ziele wurden neun Szenarien modelliert, welche Interaktionen mit dem Probandenmanagementsystem beschreiben. Ziele und Szenarien sowie eine Literaturrecherche dienten anschließend zur Identifizierung der lösungsorientierten Anforderungen. Insgesamt wurden 30 funktionale Anforderungen, sieben Qualitätsanforderungen und neun Randbedingungen identifiziert.

Die identifizierten Anforderungen bildeten den Ausgangspunkt zur Entwicklung des prototypischen Probandenmanagementsystems. Durch den entwickelten Prototyp werden 23 funktionale Anforderungen, zwei Qualitätsanforderungen sowie vier Randbedingungen direkt erfüllt. Die übrigen Anforderungen konnten aufgrund ihrer Komplexität oder wegen fehlender organisatorischer Randbedingungen nicht erfüllt werden.

In der nachfolgenden Validierung wurde geprüft, inwieweit das Probandenmanagementsystem die Erwartungen der Stakeholder erfüllt. Um dies zu untersuchen wurde ein Usability-Test mit zehn Stakeholdern durchgeführt. Insgesamt bewerteten die Stakeholder das entwickelte System sehr positiv. Alle Stakeholder brachten im Anschluss an den Usability-Tests den Wunsch zum Ausdruck, das Probandenmanagementsystem für ihre Forschungsprojekte zeitnah nutzen zu können. Aktuell wird das System in drei Forschungsprojekten an der Universitätsmedizin Göttingen eingesetzt.

In dieser Dissertation konnte so gezeigt werden, dass mit der Einführung eines Probandenmanagementsystems der Bedarf an effizienter und einheitlicher Verwaltung sowie langfristiger Nachverfolgung von Probanden erfüllt wird. 


\begin{abstract}
There is a demand for efficient, uniform, and long-term management of participants in medical research projects. In Germany, this demand originates from the "bench-to-bedside"-approach, the increase in funding of collaborative research projects, and the establishing of new scientific institutes; e.g. biobanks in university hospitals.

This thesis analyzes whether the implementation of a participant management system in University Medical Center Goettingen can meet the demand for an efficient, uniform, and longterm management of participants. First, a requirement analysis was performed, based on 17 interviews with stakeholders at University Medical Center Goettingen. The stakeholder group consisted of physicians, research associates, study nurses, and IT-professionals. The interviews yielded the identification of 16 goals to be fulfilled by the participant management system. Based on these goals, nine scenarios, which describe interactions with the participant management system, were modelled. The goals and scenarios, as well as a literature analysis, were used to elicit solution-oriented requirements for the participant management system. In total, 30 functional requirements, seven qualitative requirements, and nine constraints were formulated.
\end{abstract}

The requirements and constraints were the starting point for the development of a software prototype. The developed prototype directly fulfilled 23 functional requirements, two qualitative requirements, and four constraints. The remaining requirements and constraints could not be fulfilled due to their complexity or as a result of organizational limitations.

A consecutive evaluation process determined whether the software prototype for a participant management system met the stakeholders' expectations. Therefore, a usability test with ten stakeholders was performed. The stakeholders gave the prototype an overall very positive rating. All stakeholders expressed their wish to implement the prototype in their own research projects as soon as possible. Currently, the developed participant management system is used in three research projects in University Medical Center Goettingen.

This thesis shows that the demand for an efficient, uniform, and long-term management of participants in medical research projects can be met by the implementation of participant management system. 


\section{Danksagung}

An dieser Stelle möchte ich mich bei den beiden Betreuern meiner Dissertation Prof. Dr. Otto Rienhoff und Prof. Dr. Ramin Yahyapour bedanken. Beide standen mir mit Rat und Tat zur Seite, ebenso wie die weiteren Mitglieder meines Thesis Komitees, bestehenden auf Prof. Dr. Dieter Hogrefe und Prof. Dr. Ulrich Sax. In diesem Zusammenhang möchte ich mich auch bei Prof. Dr. Rolf Bendl und Prof. Dr. Tim Friede bedanken, die sich bereiterklärt haben, als Mitglieder der Prüfungskommission meiner Dissertation zu fungieren.

Meine Dankbarkeit gilt auch den Mitarbeitern der Universitätsmedizin Göttingen, die ich im Rahmen meiner Dissertation interviewen durfte und die am Usability-Test teilgenommen haben.

Besonderer Dank gilt Thomas Franke, Dieter Grimmig, Christoph Jensen und Dr. Bernd Schütze für die ehrlichen und offenen Anmerkungen. Außerdem danke ich auch Christian Bauer, Philipp Kachel sowie Benjamin Löhnhardt für die vielen aufmunternden Gespräche und auch Wolfgang Frase für die letzten Anregungen kurz vor Abgabe.

Ich danke meinen Eltern für die fortwährende Unterstützung. Danke auch an meine Schweigereltern, welche mir ermöglichten in der Abgeschiedenheit ihres Hauses den größten Teil meiner Dissertation zu verfassen. Abschließend möchte ich meiner Frau danken, ohne Sie wäre diese Dissertation nicht möglich gewesen. 


\section{Inhaltsverzeichnis}

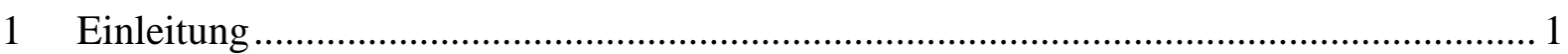

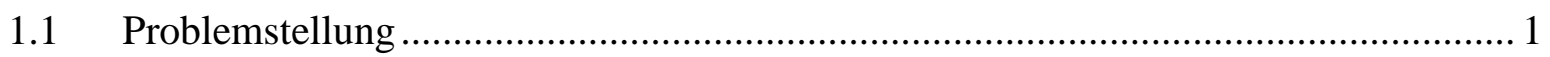

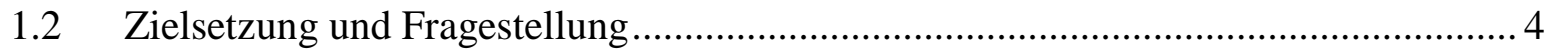

1.3 Aktueller Stand der Forschung und Technik ....................................................... 6

1.4 Aufbau und Struktur der Arbeit ........................................................................ 7

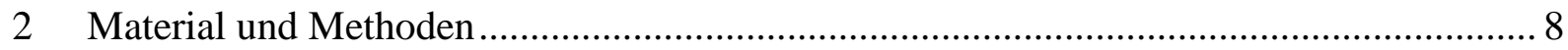

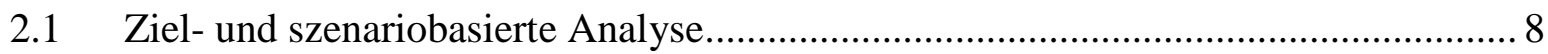

2.2 Ermittlung der lösungsorientierten Anforderungen................................................. 12

2.3 Entwicklung des Architekturkonzepts ............................................................. 14

2.4 Prototypische Implementierung des Architekturkonzepts...................................... 18

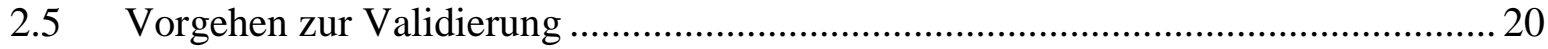

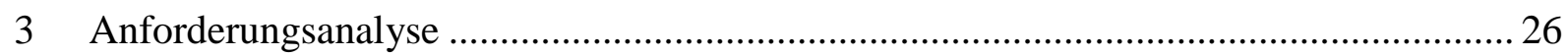

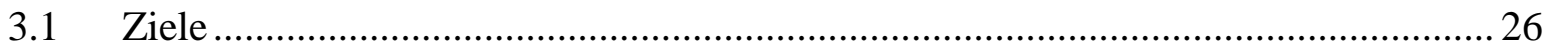

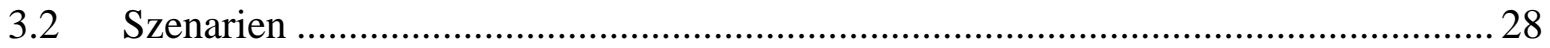

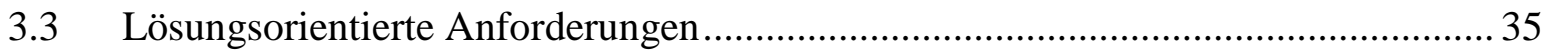

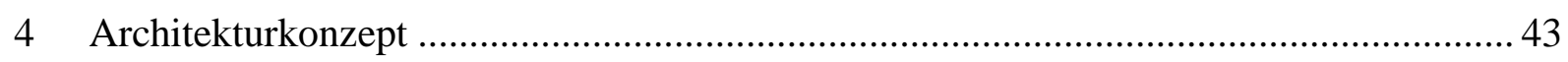

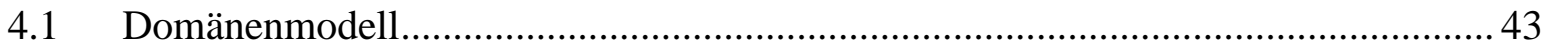

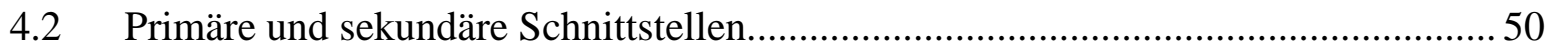

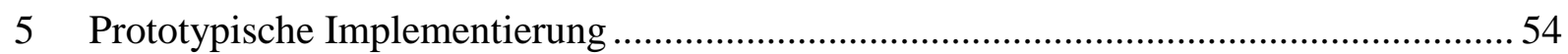

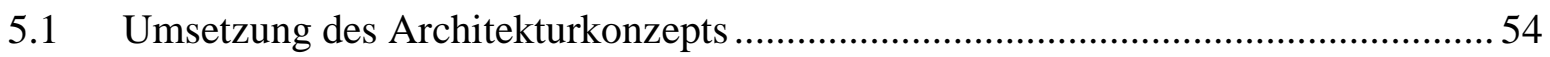

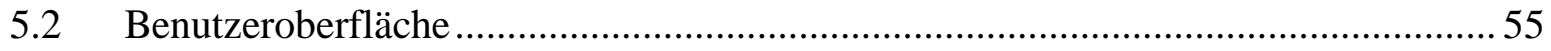

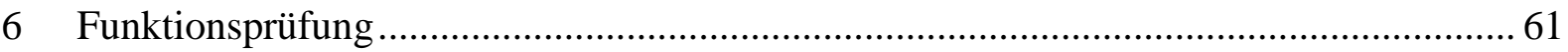

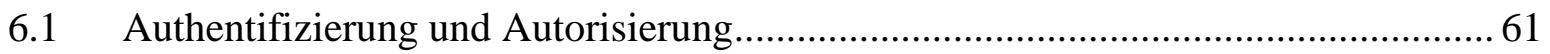

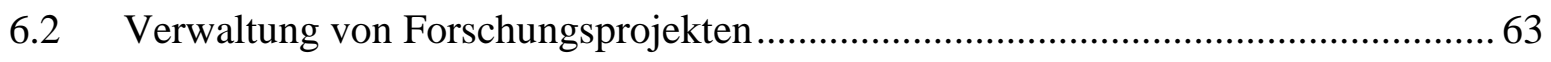

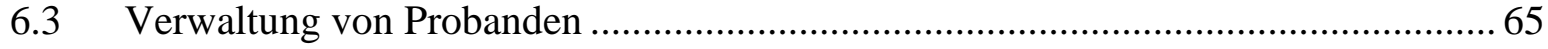

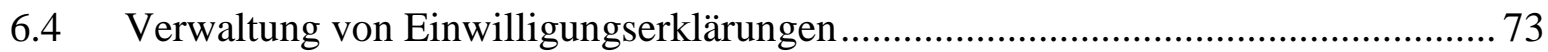

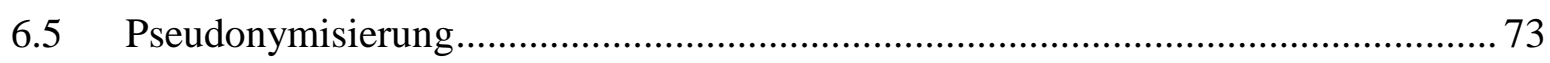

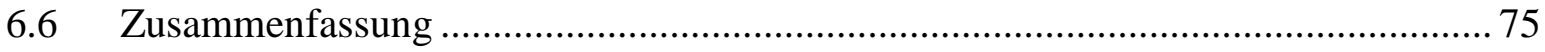

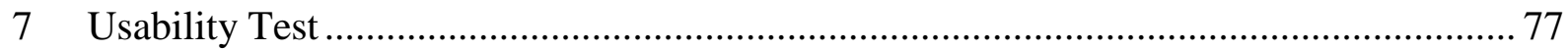

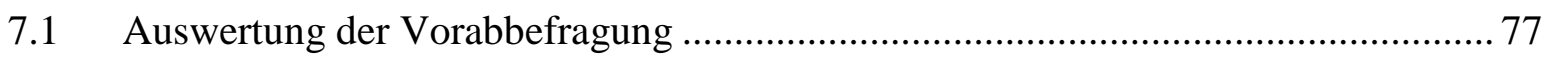

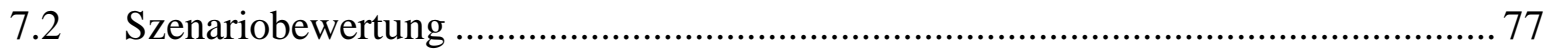

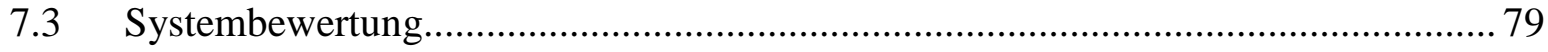




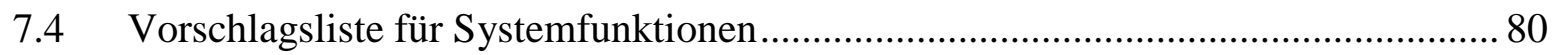

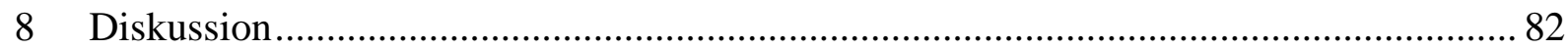

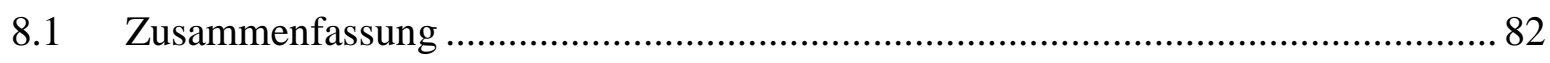

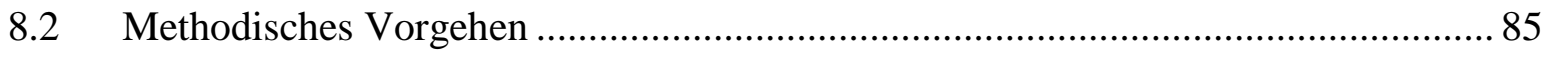

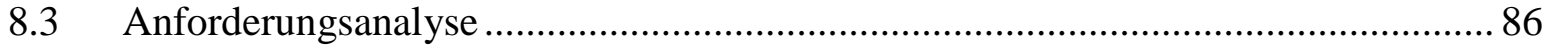

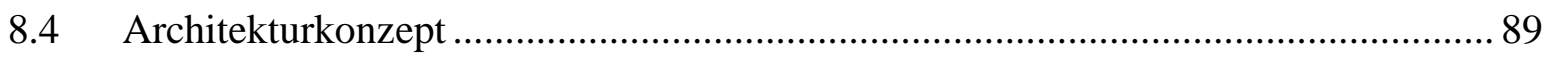

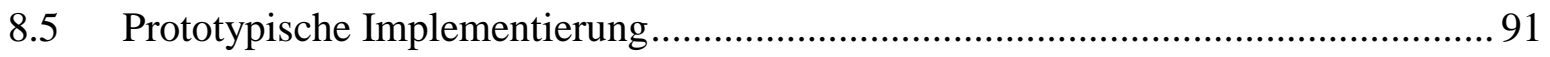

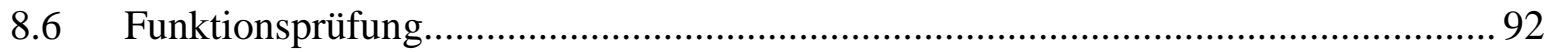

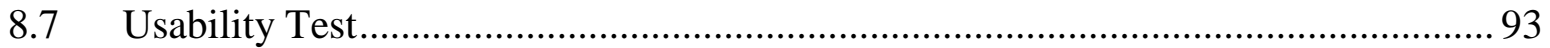

8.8 Einordnung des Probandenmanagements im Forschungsumfeld ............................ 95

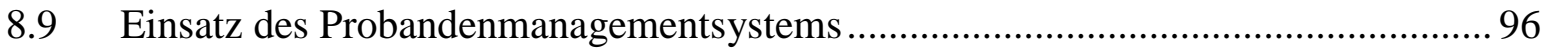

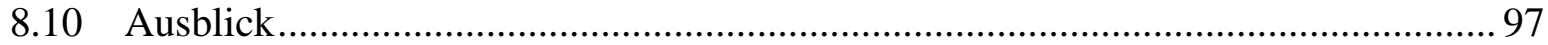

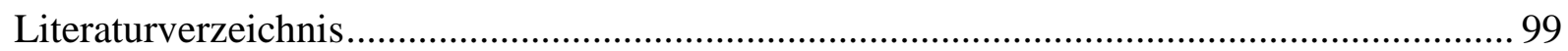

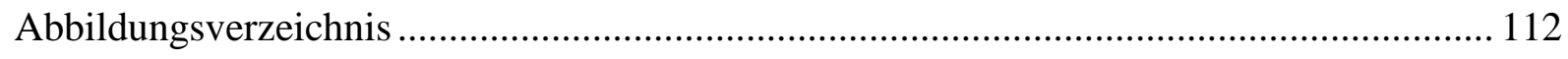

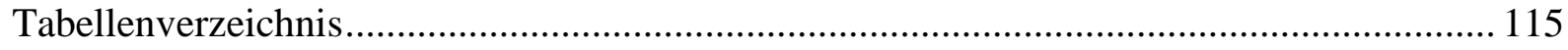

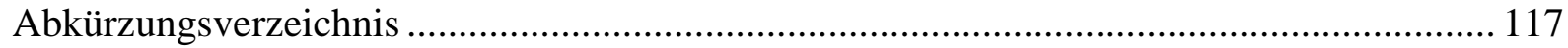

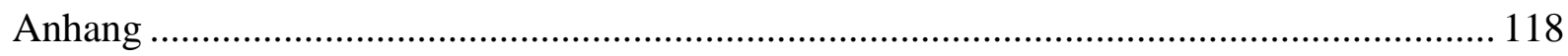

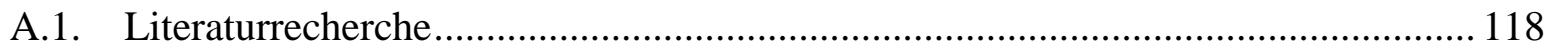

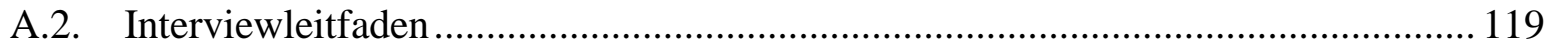

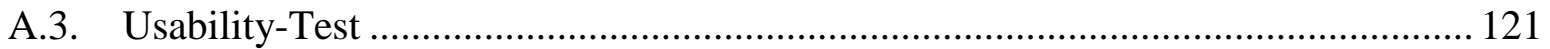

A.4. Berechnung des Scores für den System Usability Scale ..................................... 127

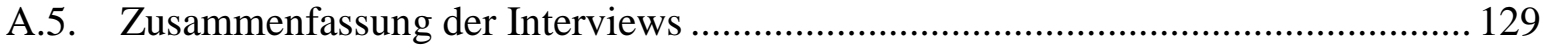

A.6. Ergänzungen zum Architekturkonzept ............................................................... 137

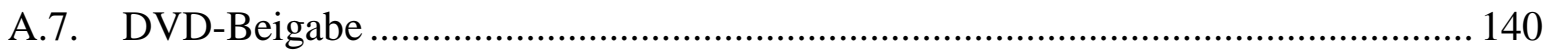

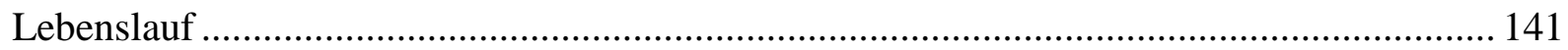




\section{Einleitung}

In medizinischen Forschungsprojekten besteht großer Bedarf an einem Probandenmanagement. Dies umfasst die effiziente und einheitliche Verwaltung sowie die langfristige Nachverfolgung von Probanden in Forschungsprojekten [1, 2]. Dieser Bedarf wird im Abschnitt 1.1 auf Grundlage von Beispielen hergeleitet und im darauffolgenden Abschnitt 1.2 zur Entwicklung der Forschungsfragestellungen dieser Dissertation verwendet. Abschnitt 1.3 beschreibt den aktuellen Stand der Forschung und Technik im Bereich des Probandenmanagements. Innerhalb des letzten Abschnitts 1.4 wird der Aufbau und die Struktur der vorliegenden Dissertation erläutert.

\subsection{Problemstellung}

Die Anforderungen an ein Probandenmanagement ergeben sich aus den drei Kernpunkten Effizienz, Einheitlichkeit und Langfristigkeit. Diese werden nachfolgend anhand von Beispielen aus der medizinischen Forschung in Deutschland dargestellt.

\subsubsection{Effizienz}

Der Bedarf an einer effizienten Verwaltung von Probanden wird am Beispiel von genomweiten Assoziationsstudien, Registern für seltene Erkrankungen und an klinischen Studien erläutert.

Das primäre Ziel genomweiter Assoziationsstudien ist es, Variationen im Genom des Menschen zu identifizieren und Assoziationen zu bestimmten Phänotypen herzustellen, bspw. zu einer bipolaren Störung [3]. Abhängig von der spezifischen Fragestellung sind bisweilen große Kollektive mit bis zu 200.000 Probanden notwendig, um signifikante Forschungsergebnisse zu erzielen [4]. Die Rekrutierung solch großer Probandenkollektive ist nur durch Forschungskooperationen auf internationaler Ebene möglich [5]. Beispielhaft zeigt dies ein internationales Forschungsprojekt mit über 180.000 Probanden zur Analyse von Genomvarianten im Hinblick auf die Körpergröße [6]. Die (internationale) Koordination, die Rekrutierung sowie die Durchführung der Untersuchungen an den Probanden erfordert ein effizientes Management [7].

Ein weiteres Beispiel für den Bedarf an einer effizienten Verwaltung von Probanden sind medizinische Register für seltene Erkrankungen. Gemäß Verordnung (EG) Nr. 141/2000 der Europäischen Union gilt eine Erkrankung als selten „,...], wenn nicht mehr als 5 von 10.000 Menschen an ihr leiden [...]“ [8, S. 1]. Dies ist für die medizinische Forschung eine große Herausforderung, da durch die geringen Patientenzahlen kaum statistisch signifikante Aussagen über die Behandlung möglich sind [9]. Ein wichtiges Ziel der Register für seltene Erkrankungen ist daher eine möglichst vollständige Erfassung der Betroffenen, welche an einer seltenen Erkrankung leiden [10-12]. Eine langjährige Kontaktpflege zu den betroffenen Personen ist daher für die Forschung essentiell [13].

Das letzte Beispiel umfasst den Bedarf an einer effizienten Verwaltung von Probanden aus Sicht klinischer Studien [14]. So erreicht beispielsweise nur jede zweite klinische Studie die geplante Fallzahl von Probanden in der vorgegebenen Zeit [15]. In diesem Zusammenhang ist 
auch die immer stärker werdende Vernetzung der klinischen Forschung mit der Patientenversorgung zu betrachten. Ziel dieser Vernetzung ist zum einen, mehr Patienten zu rekrutieren, und zum anderen, die (sekundäre) Nutzung von Versorgungsdaten in der Forschung voranzubringen [16-18]. Ein Beispiel für diese sekundäre Nutzung von Daten stammt aus dem Bereich der Onkologie. Neben klinischen Parametern werden auch Daten über Lebensqualität und eventuelle Spätfolgen einer Erkrankung erfasst [19]. Diese können für die Forschung genutzt werden, um beispielsweise eine Aussage über die Lebensqualität nach einer spezifischen onkologischen Behandlung zu treffen. Klinische Studien benötigen daher eine effiziente Verwaltung von Probanden bzw. Patienten im Hinblick auf eine kontinuierliche Kontaktpflege auch über mehrere Jahre [20] sowie eine Verbindung zwischen der Identität des Patienten im Krankenhaus und der des Probanden in Forschungsprojekten zur Sicherstellung der Vernetzung zwischen Forschung und Versorgung [16].

Zusammenfassend zeigen die drei Beispiele, dass der Bedarf nach einer effizienten Verwaltung von Probanden für verschiedene Typen von Forschungsprojekten unterschiedliche Ausprägungen aufweist. So müssen in genomweiten Assoziationsstudien eine Vielzahl von Probanden verwaltet werden. In medizinischen Registern für seltene Krankheiten ist zwar die Zahl der Probanden geringer, aber eine wesentlich intensivere Kontaktpflege notwendig. Klinische Studien benötigen hingegen eine enge Verbindung zwischen der Identität des Patienten im Krankenhaus und der des Probanden in Forschungsprojekten.

\subsubsection{Einheitlichkeit}

Die immer stärkere Vernetzung der medizinischen Forschung - vor allem in Deutschland bildet die Grundlage für den Bedarf nach einer einheitlichen Verwaltung von Probanden. Dieser Bedarf wird am Beispiel der vernetzen deutschen Gesundheitsforschung und an spezialisierten Softwaresystemen zur Verwaltung von Probanden in der vernetzten Gesundheitsforschung erläutert.

Ausgangspunkt sind die Kompetenznetze in der Medizin, deren Ziel die vertikale und horizontale Vernetzung der deutschen Gesundheitsforschung ist [21]. Die vertikale Vernetzung beschreibt den Transfer von Ergebnissen aus der Forschung in die Patientenversorgung [22]. In einem anderen Zusammenhang wird dies auch als translationale Forschung bezeichnet [23-28]. Im Vergleich zur vertikalen Vernetzung bündelt die horizontale Vernetzung vorhandene Kompetenzen einzelner Forschungseinrichtungen, wie z. B. Universitätskliniken, in einem Forschungsverbund mit dem Ziel gemeinsame Lösungen für wissenschaftliche Fragestellungen zu erarbeiten [29-35]. Die erfolgreiche Evaluation der medizinischen Kompetenznetze und der Rahmenplan für die Gesundheitsforschung des Bundesministeriums für Bildung und Forschung zeigen, dass die Zukunft der deutschen Gesundheitsforschung in der Vernetzung von Forschungseinrichtungen liegt [36]. Forscher werden daher zukünftig in immer mehr Forschungsverbünden involviert sein [37-40]. 
Ein wichtiges Beispiel für spezialisierte Softwaresysteme für die Vernetzung der medizinischen Forschung ist die zentrale Erfassung und Pseudonymisierung von Probandendaten [41]. Pseudonymisierung beschreibt einen Prozess, in welchem die personenbezogenen Daten einer Person (Name, Geburtsdatum, Geschlecht etc.) durch ein Pseudonym ersetzt werden. Im Vergleich zur Anonymisierung bleibt ein Rückschluss auf die personenbezogenen Daten möglich [42].

In einer Kooperation zwischen dem Kompetenznetz Pädiatrische Onkologie und Hämatologie, dem Institut für Medizinische Biometrie, Epidemiologie und Informatik an der Universitätsmedizin Mainz und der Technologie- und Methodenplattform für die vernetzte medizinische Forschung e. V. (TMF) wurde im Jahr 2000 das erste Softwaresystem zur zentralen Erfassung und Pseudonymisierung von Probandendaten in Deutschland entwickelt [43]. Dieses Softwaresystem (PIDGenerator) ermöglicht die zentrale Erfassung von personenbezogenen Probandendaten sowie deren Pseudonymisierung über eine Weboberfläche. Das zugrundeliegende Konzept wurde in verschiedenen Versionen von weiteren Forschungsprojekten aufgegriffen und an deren spezifische Anforderungen angepasst [2, 44-47].

Ein Workshop zum Thema Verwaltung von Probanden am 12. Juni 2013 in Göttingen zeigte, dass in Deutschland mittlerweile verschiedene Softwarelösungen mit unterschiedlichem Funktionsumfang zur Erfassung und Pseudonymisierung von Probanden in medizinischen Forschungsprojekten existieren [48]. So nutzen beispielsweise vier der sechs deutschen Zentren der Gesundheitsforschung [49] unterschiedliche Softwareanwendungen zur Pseudonymisierung von Probanden [50-53]. Auch international gibt es in diesem Bereich Softwarelösungen, welche spezifisch für einzelne Forschungsprojekte entwickelt wurden [41, 44, 54-58].

Die immer stärkere Vernetzung der medizinischen Forschung führt dazu, dass Forscher in immer mehr Verbundforschungsprojekten involviert werden. Innerhalb eines Forschungsverbundes wiederum müssen die Probanden über mehrere Forschungseinrichtungen hinweg durch den Einsatz eines Softwaresystems zentral erfasst und pseudonymisiert werden. Die organisatorischen Rahmenbedingungen der einzelnen Forschungsprojekte und deren unterschiedliche Softwaresysteme führen dazu, dass eine projektübergreifende Sicht eines Forschers auf seine Probanden und deren einheitliche Verwaltung nicht mehr möglich ist. Daher besteht bei Forschern einer Forschungseinrichtung ein großer Bedarf ihre Probanden einheitlich über verschiedene Forschungsprojekte und -verbünde zu verwalten.

\subsubsection{Langfristigkeit}

Der letzte Kernpunkt umfasst die langfristige Nachverfolgung von Probanden. Dieser Bedarf an Langfristigkeit wird am Beispiel von Biobanken und Registern für chronische Erkrankungen dargestellt.

An verschiedenen Forschungseinrichtungen werden zentrale Biobanken aufgebaut [2, 59-61]. Diese sammeln und verarbeiten qualitativ hochwertige Biomaterialproben im Rahmen der Pa- 
tientenbehandlung. Dies ist notwendig, da Biomaterialien zum unverzichtbaren Teil der medizinischen Forschung geworden sind [62]; so erfordert die Analyse des menschlichen Genoms die Entnahme von Biomaterialien, wie beispielsweise Blut, Urin oder Gewebe. Ohne eine ausreichende Anzahl an qualitativ hochwertigen Biomaterialien ist die Durchführung von Forschungsprojekten nur schwer möglich [63, 64]. Die Biobanken erfassen zur besseren Qualifizierung des Biomaterials zusätzliche medizinische Daten über die jeweiligen Patienten, z. B. über seine Behandlung [65]. Diese Kombination aus Biomaterial und medizinischen Daten wird dann einzelnen Forschungsprojekten zur Verfügung gestellt [66], da Biomaterialien aus dem Fundus einzelner Forscher heutzutage im Hinblick auf Menge und Qualität nicht mehr ausreichend sind [3, 67]. Generell erfordert die Entnahme und Einlagerung von Biomaterialien eine Einwilligung des Patienten bzw. Probanden. Die Weitergabe dieser Biomaterialien an Forschungsprojekte erfordert dann meist eine erneute Einwilligung, da eine Zweckänderung vorliegt. Dies erfordert eine langfristige Nachverfolgung von Patienten bzw. Probanden, wozu eine erneute Kontaktierung möglich sein muss. Im Sinne des „Biobanking 3.0“ besteht ebenso ein Bedarf, Probanden aufzuzeigen, in welchen Forschungsprojekten ihre Biomaterialien und medizinischen Daten verwendet werden [68].

Ein weiteres Beispiel für den Bedarf an einer langfristigen Nachverfolgung von Probanden bilden Forschungsprojekte im Bereich der chronischen Erkrankungen wie z. B. Multiple Sklerose. Multiple Sklerose führt zur allmählichen Demyelinisation des Nervensystems und wird meist im Lebensalter von 20 bis 40 Jahren diagnostiziert [69]. Bei Anwendung der aktuellen Behandlungsmethoden verkürzt sich die durchschnittliche Lebenszeit bei Männern um 11,6 Jahre und bei Frauen um 16,4 Jahre [69]. Zur Behandlung von Multipler Sklerose sind regelmäßige Facharztbesuche mit diagnostischen Untersuchungen notwendig. Innerhalb von Forschungsprojekten werden die Betroffenen über einen sehr langen Zeitraum, teilweise bis zu ihrem Lebensende, beobachtet. Dies erfordert eine langfristige Nachverfolgung der Betroffenen über mehrere Jahrzehnte [70].

Die beiden Beispiele zeigen, dass die langfristige Nachverfolgung von Probanden eine Notwendigkeit in Forschungsprojekten darstellt. Biobanken müssen die erneute Kontaktierung von Probanden sicherstellen und den Probanden aufzeigen, in welchen Forschungsprojekten ihre Daten und Biomaterialien genutzt werden. Im Bereich der Multiple Sklerose-Forschung ist es wichtig, über mehrere Jahrzehnte den Kontakt zu den Probanden aufrecht zu erhalten. Innerhalb einer solchen Zeitspanne sind der Wechsel und das Ausscheiden von Forschern eines Forschungsprojektes durchaus realistisch, sodass die Nachverfolgbarkeit auch über mehrere Forschergenerationen hinweg sichergestellt sein muss.

\subsection{Zielsetzung und Fragestellung}

In der Problemstellung wurde gezeigt, dass ein großer Bedarf an einem Probandenmanagement in medizinischen Forschungsprojekten besteht. Das Probandenmanagement muss eine effiziente und einheitliche Verwaltung sowie eine langfristige Nachverfolgung von Probanden über 
mehrere medizinische Forschungsprojekte an einer Forschungseinrichtung sicherstellen. Folglich ist die Kernaufgabe des Probandenmanagements die Bereitstellung der richtigen Informationen zum richtigen Zeitpunkt, am richtigen Ort, in der richtigen Form und für die richtigen Personen [1]. Das Probandenmanagement soll also die Informations- und Wissenslogistik über Probanden einer Forschungseinrichtung, äquivalent zum Krankenhausinformationssystem über Patienten, gewährleisten [71]. Aus dieser Schlussfolgerung leitet sich die zentrale Forschungshypothese dieser Dissertation ab:

Forschungshypothese: Die Etablierung eines Informationssystems zum Probandenmanagement (Probandenmanagementsystem) an einer Forschungseinrichtung vermag die effiziente und einheitliche Verwaltung sowie die langfristige Nachverfolgung von Probanden über mehrere medizinische Forschungsprojekte sicherzustellen.

Vor der eigentlichen Etablierung des Systems steht dessen Konzeption und Umsetzung. Die Grundlage zur Konzeption bilden Szenarien, welche die Interaktionen mit dem Probandenmanagementsystem basierend auf den drei Kernpunkten Effizienz, Einheitlichkeit und Langfristigkeit beschreiben. Die Ermittlung der Szenarien erfolgt am Beispiel der Universitätsmedizin Göttingen, da diese Dissertation im Rahmen einer wissenschaftlichen Anstellung am Institut für Medizinische Informatik der Universitätsmedizin Göttingen verfasst wurde. Daraus ergibt sich die erste Forschungsfragestellung:

Forschungsfrage 1: Welche Szenarien existieren für den Einsatz des zu konzeptionierenden Probandenmanagementsystems an der Universitätsmedizin Göttingen?

Basierend auf den Szenarien erfolgt im nächsten Schritt die Konzeption des Probandenmanagementsystems. Dabei sollen nicht nur die Szenarien berücksichtigt werden, sondern auch die existierenden Softwaresysteme zur zentralen Erfassung und Pseudonymisierung von Probanden in Verbundforschungsprojekten. Zur Sicherstellung der Einheitlichkeit sollen geeignete Schnittstellen im Rahmen der Konzeption modelliert werden, um Synergien zu existierenden Softwaresystemen nutzbar zu machen. Nach Abschluss der Konzeption erfolgt die prototypische Implementierung eines Probandenmanagementsystems. Diese soll die Umsetzbarkeit des Konzepts und der modellierten Schnittstellen aufzeigen. Die zweite Forschungsfragestellung dieser Dissertation lautet daher wie folgt:

Forschungsfrage 2: Wie kann ein Probandenmanagementsystem unter Berücksichtigung der Szenarien und Schnittstellen konzipiert und prototypisch implementiert werden?

Zusammenfassend ist das Ziel dieser Dissertation, den Forschern an der Universitätsmedizin Göttingen ein Werkzeug bereitzustellen, um eine effiziente und einheitliche Verwaltung sowie eine langfristige Nachverfolgung von Probanden über mehrere medizinische Forschungsprojekte sicherzustellen. 


\subsection{Aktueller Stand der Forschung und Technik}

Basierend auf einer Literaturrecherche wurde der aktuelle Stand der Forschung und Technik im Bereich des Probandenmanagements ermittelt (siehe Anhang A.1). Innerhalb der Literaturrecherche wurden drei Schwerpunkte identifiziert: „Sekundärnutzung“, „Pseudonymisierung“ und „Prozessunterstützung“.

Ziel der Sekundärnutzung ist es, Daten aus der Patientenversorgung für die medizinische Forschung zugänglich und nutzbar zu machen [16]. Ein Teilbereich der Sekundärnutzung ist die Rekrutierungsunterstützung, deren Ziel es ist, potentielle Probanden für Forschungsprojekte zu identifizieren [72-74]. Dies erfolgt beispielsweise auf Grundlage der medizinischen Daten eines Patienten im Krankenhausinformationssystem. Diese Daten werden automatisch gegen die Ein- und Ausschlusskriterien der einzelnen Forschungsprojekte geprüft [75]. Die behandelnden Ärzte werden nachfolgend über mögliche Forschungsprojekte informiert, diese wiederum informieren Patienten über die möglichen Projekte [76]. Sollten die Patienten einverstanden sein, wird das Forschungsprojekt informiert.

Ein weiteres Beispiel aus dem Bereich der Sekundärnutzung von Patientendaten ist die Verwendung medizinischer Daten aus der elektronischen Patientenakte des jeweiligen Patienten für die Forschung [77-80]. Dies erfordert eine enge Vernetzung zwischen Versorgung und Forschung unter Berücksichtigung des Datenschutzes [81-85]. In der Versorgung ist die Identität des Patienten bekannt, wohingegen in der Forschung die Identität durch ein Pseudonym repräsentiert wird.

Die Pseudonymisierung ist ein wichtiges Vorgehen zur Sicherstellung des Datenschutzes in medizinischen Forschungsprojekten [47]. Eine große Herausforderung im Bereich der Pseudonymisierung ist die Erkennung von identischen bzw. ähnlichen Personen, dies wird als Duplikaterkennung bezeichnet [43]. Die verschiedenen Softwaresysteme zur Pseudonymisierung nutzen unterschiedliche Algorithmen zur Duplikaterkennung. Diese können mitunter sehr einfach sein und nur auf Basis von Übereinstimmung prüfen [44, 53], Phonetik berücksichtigen [43, 45] oder komplexe Berechnungen vornehmen [86, 87].

Im Bereich der Prozessunterstützung existiert eine große Anzahl von Studienmanagementsystemen, die meist gemäß der spezifischen Anforderung einzelner Forschungsprojekte entwickelt wurden [88-93]. Vornehmlich dienen die Systeme der Erfassung von Forschungsdaten sowie dem Projektmanagement (z. B. Kostenplanung) [94, 95]. Hinzu kommen Funktionen zur Planung von Aufgaben, bspw. Untersuchungen von Probanden. Dies wird teilweise auch als „Tracking“ bezeichnet [91, 96-98].

Generell zeigte die Literaturrecherche, dass unterschiedliche Systeme im Bereich des Probandenmanagements existieren. Das Verhältnis der existierenden Systeme zum zu konzeptionierenden Probandenmanagementsystem wird im Kapitel 8 dieser Dissertation erneut aufgegriffen und diskutiert. 


\subsection{Aufbau und Struktur der Arbeit}

Diese Dissertation ist unterteilt in vier Bereiche: Einleitung, Material und Methoden, Ergebnisse und Diskussion. Die Einleitung (Kapitel 1) beschreibt die Problemstellung, Zielsetzung sowie die Fragestellung der vorliegenden Arbeit. Innerhalb von Kapitel 2 wird die Methodik zur Beantwortung der beiden Forschungsfragen beschrieben. Die Ergebnisse dieser Dissertation wurden zur besseren Lesbarkeit in vier Kapitel unterteilt: Anforderungsanalyse (Kapitel 3), Architekturkonzept (Kapitel 4), prototypische Implementierung (Kapitel 5) und Validierung (Kapitel 6 und 7). Den Abschluss der Arbeit bildete das Kapitel 8, in welchem die Inhalte der Dissertation kritisch analysiert werden. 


\section{Material und Methoden}

Gegenstand des ersten Abschnitts (2.1) der Methodik ist die Beschreibung des Vorgehens zur Ermittlung der Szenarien für den Einsatz des zu konzeptionierenden Probandenmanagementsystems an der Universitätsmedizin Göttingen (Forschungsfrage 1). Abschnitt 2.2 bildet den Übergang von der ersten zur zweiten Forschungsfrage und beschreibt die angewendete Methodik zur Ermittlung von Anforderungen für die Konzeption und die prototypische Implementierung des Probandenmanagementsystems. Die zugrundeliegenden Materialien und Methoden für die Konzeption und prototypische Implementierung (Forschungsfrage 2) sind Teil der Abschnitte 2.3 und 2.4. Abschließend beschreibt Abschnitt 2.5 das Vorgehen zur Validierung der prototypischen Implementierung mit Hilfe eines Usability-Tests.

\subsection{Ziel- und szenariobasierte Analyse}

Die erste Forschungsfragestellung umfasst die Ermittlung der Szenarien für den Einsatz des zu konzeptionierenden Probandenmanagementsystems an der Universitätsmedizin Göttingen. Die zu identifizierenden Szenarien beschreiben die notwendigen Interaktionen mit dem Probandenmanagementsystem zur Erfüllung eines Ziels [99]. Die Ermittlung der Szenarien erfordert daher zunächst die Identifizierung von Zielen [100-102].

Ein Ziel ist definiert als die intentionale Beschreibung eines charakteristischen Merkmals des zu konzeptionierenden bzw. zu entwickelnden Informationssystems [99, 103]. Ziele repräsentieren die Intentionen von Personen, die ein besonderes Interesse am Probandenmanagementsystem sowie einen direkten oder indirekten Einfluss auf dessen Gestaltung haben (Stakeholder) [104-106]. Die Identifizierung der Ziele erfolgt daher in Zusammenarbeit mit den Stakeholdern unter Anwendung von leitfadengestützten Interviews.

Das Vorgehen zur Identifizierung von Szenarien auf der Grundlage von Zielen bietet den Vorteil, dass die Stakeholder bei der Ermittlung der Szenarien durch deren Ziele berücksichtigt werden. Darüber hinaus kann nachvollziehbar aufgezeigt werden, aus welchen Zielen die entsprechenden Szenarien hergeleitet wurden. Folglich existiert kein Szenario ohne assoziiertes Ziel. Ein weiterer Vorteil der Verwendung von Zielen und Szenarien ist, dass diese als Grundlage zur Ermittlung der Anforderungen an das Probandenmanagementsystem dienen [99, 102]. Die Anforderungen werden für die Konzeption und prototypische Implementierung des Systems benötigt (siehe Abschnitt 2.2).

\subsubsection{Interviews mit Stakeholdern}

Die primäre Zielsetzung der Interviews war es, die Intention und Erwartung der Stakeholder an ein Probandenmanagementsystem zu eruieren. Dies erfolgt unter Anwendung eines leitfadengestützten Interviews. Ein leitfadengestütztes Interview ist ein offenes Gespräch über einen Sachverhalt anhand eines Interviewleitfadens [107]. Der Interviewer kann vom vorgegebenen Leitfaden abweichen und so besser auf den Interviewten eingehen [108]. Offene Punkte können 
direkt geklärt oder hinterfragt werden, wodurch beispielsweise innovative Ideen und Anforderungen ermittelt werden können [99].

Insgesamt wurden 17 Stakeholder interviewt, um ein breites Spektrum an Intentionen und Erwartungen zu erfassen. Die Auswahl der Stakeholder erfolgte auf Grundlage von Schwanke et al. [1]. Die dortige Stakeholdergruppe bestand aus Ärzten, Studienassistenten, Mitarbeitern der Krankenhaus-IT sowie wissenschaftlichen Mitarbeitern. Diese Zusammensetzung bildete den Ausgangspunkt für die Dissertation. Insgesamt umfasste die Stakeholdergruppe sechs Ärzte, vier Mitarbeiter der Krankenhaus-IT, drei Studienassistenten, drei wissenschaftliche Mitarbeiter sowie den behördlichen Datenschutzbeauftragten der Universitätsmedizin Göttingen. Alle Stakeholder sind Mitarbeiter der Universitätsmedizin Göttingen und Kooperationspartner des Instituts für Medizinische Informatik. Durch die vorangegangen Kooperationen mit den Stakeholdern, bspw. durch gemeinsame Forschungsprojekte, konnte sichergestellt werden, dass alle ausgewählten Personen potentielle Stakeholder für ein Probandenmanagementsystem sind.

Entsprechend dem Interviewleitfaden (siehe Anhang A.2) wurden zu Beginn des Interviews die Zielsetzung dieser Dissertation und des Interviews erläutert. Nachfolgend wurden allgemeine Informationen über die Stakeholder erfragt. Stakeholdern, die einen direkten Kontakt zu Probanden haben, werden zusätzliche Fragen zur Beteiligung an medizinischen Forschungsprojekten und zum Umgang mit Probanden gestellt. Anschließend wurde den Stakeholdern eine Vorversion des Probandenmanagementsystems präsentiert [109]. Diese Vorversion wurde vor dem eigentlichen Beginn dieser Dissertation entwickelt und bildete die Grundlage zur Entwicklung der Forschungshypothese sowie der beiden Forschungsfragestellungen. Sie verfügt nur über einen rudimentären Funktionsumfang und wurde nicht als projektübergreifendes System ausgelegt, sondern dient nur zur Verwaltung von Probanden innerhalb eines Forschungsprojekts. Durch die Vorversion sollte den Stakeholdern ein erster Eindruck eines Probandenmanagementsystems ermöglicht werden [108]. Zur Vermeidung einer Beeinflussung der Stakeholder wurde die Vorversion des Probandenmanagementsystems erst nach der eigentlichen Befragung vorgestellt.

Der Interviewleitfaden wurde in drei Testinterviews, deren Dauer zwischen 30 und 45 Minuten lag, evaluiert. Eine Anpassung des Leitfadens war nach der Evaluation nicht notwendig. Es zeigte sich jedoch, dass ein schriftliches Protokollieren für das Interview nicht förderlich ist. Daher wurden alle 17 Interviews elektronisch aufgezeichnet, sodass die Aufmerksamkeit des Interviewers dem Gespräch gilt und nicht dem schriftlichen Protokoll [108].

\subsubsection{Transkription und Auswertung der Interviews zur Zielgewinnung} Innerhalb der Interviews wurden Informationen zu den Erwartungen der Stakeholder an ein Probandenmanagementsystem eruiert. Diese Informationen bildete die Grundlage zur Gewinnung der Ziele, da diese die Intentionen der Stakeholder widerspiegeln [99]. 
Die Auswertung der Interviews erfolgte mittels der qualitativen Inhaltsanalyse [107]. Diese aus den Sozialwissenschaften stammende Methode beschreibt das Vorgehen zur systematischen Analyse von Textinhalten unter Berücksichtigung einer zentralen Fragestellung [110, 111]. Die qualitative Inhaltsanalyse bietet sich an, da eine zentrale Fragestellung der Interviews gegeben ist. Diese ist als die Erwartung der Stakeholder an ein Probandenmanagementsystem definiert. Außerdem sind die Textinhalte dem Interviewleitfaden entsprechend strukturiert; dies erleichtert die systematische Analyse. Ein weiterer Vorteil der qualitativen Inhaltsanalyse ist, dass der Ausdruck und die Sprache der Stakeholder für die qualitative Inhaltsanalyse nicht relevant sind und so auf eine vollständige Transkription verzichtet werden kann [107, 112]. Da diese Dissertation nicht in einem sozialwissenschaftlichen Umfeld erfolgt, und die Interviews nicht den Kern dieser Dissertation bilden, wurde auf eine vollständige Transkription verzichtet.

Zunächst wurden im Rahmen der qualitativen Inhaltsanalyse die Aussagen der Stakeholder paraphrasiert und den Leitfragen des Interviewleitfadens zugeordnet [110]. Das Ergebnis war die strukturierte Zusammenfassung ${ }^{1}$ eines jeden Interviews. Die Zusammenfassung erfolgte auf Grundlage der in Tabelle 1 dargestellten Vorlage. In der Vorlage werden die Leitfragen des Interviews als einzelne Abschnitte dargestellt.

\begin{tabular}{l|l} 
Abschnitt & Inhalt \\
\hline Tätigkeitsbereich & Kurze Beschreibung der Tätigkeit des Stakeholders. \\
\hline Forschungserfahrung & $\begin{array}{l}\text { Angaben zu bisherigen und aktuellen Forschungsprojekten, an } \\
\text { denen der Stakeholder beteiligt ist oder war. }\end{array}$ \\
\hline Rekrutierung & $\begin{array}{l}\text { Auflistung, aus welchen Quellen Probanden für die For- } \\
\text { schungsprojekte rekrutiert werden. }\end{array}$ \\
\hline Probandenverwaltung & Kurze Beschreibung, wie Probanden aktuell verwaltet werden. \\
\hline Erwartung & $\begin{array}{l}\text { Auflistung der Erwartung an ein Probandenmanagementsys- } \\
\text { tem aus Sicht der Stakeholder. }\end{array}$ \\
\hline Sonstiges & $\begin{array}{l}\text { Angaben, die für diese Dissertation wichtig sind, z. B. Rah- } \\
\text { menbedingungen oder Funktionen für das Probandenmanage- } \\
\text { mentsystem. }\end{array}$
\end{tabular}

Tabelle 1. Vorlage zur strukturierten Zusammenfassung der Interviews

Nachfolgend wurden die durch Paraphrasierung identifizierten Erwartungen der Stakeholder an ein Probandenmanagementsystem in Form von Zielen zusammengefasst [110]. Die Paraphrasierung erfolgt einzeln und unabhängig von den anderen Interviews, sodass ähnliche oder bedeutungsgleiche Ziele existieren können [107]. Daher wurden bedeutungsgleiche Ziele zusammengefasst [107, 110]. Die Darstellung der Ziele erfolgt auf Grundlage von Tabelle 2.

\footnotetext{
${ }^{1}$ Die Interviewzusammenfassungen wurden wie folgt nummeriert: I-X. I steht für Interview; X ist die laufende Nummer des Interviews.
} 


\begin{tabular}{l|l} 
Nr. $^{2}$ & Name \\
\hline Nr. & Name des Ziels
\end{tabular}

Tabelle 2. Vorlage zur zusammenfassenden Darstellung der identifizierten Ziele

Aufgrund der hohen Arbeitsbelastung der Stakeholder wurde auf eine erneute Abstimmung im Hinblick auf die korrekte Interpretation ihrer paraphrasierten Aussagen - verzichtet.

\subsubsection{Herleitung der Szenarien aus den gewonnenen Zielen}

Ein Szenario beschreibt die Interaktion mit dem Probandenmanagementsystem zur Erfüllung eines oder mehrerer Ziele [99-101]. Diese Gegebenheit bildet die Grundlage zur Herleitung der Szenarien aus den gewonnenen Zielen: Beginnend mit dem ersten identifizierten Ziel wird ein Szenario modelliert, welches das Ziel erfüllt. Nachfolgend wird geprüft, ob das nächste Ziel durch das Szenario, gegebenenfalls durch eine Erweiterung, ebenfalls erfüllt wird. Ist dies nicht möglich, wird für dieses Ziel ein neues Szenario modelliert; für alle Ziele wird entsprechend verfahren.

\subsubsection{Modellierung der Szenarien}

Ausgangspunkte der Modellierung eines Szenarios sind die zugrundeliegenden Ziele [100, 101] und die damit verbundenen Akteure, beispielsweise ein Systemnutzer oder ein anderes System [108]. Innerhalb der Modellierung werden alle zur Zielerfüllung notwendigen Interaktionen der Akteure mit dem Probandenmanagementsystem erfasst [99]. Diese Interaktionen bilden schließlich das eigentliche Szenario. Eine reine schriftliche Beschreibung der Interaktionen in einem Szenario ist meist sehr komplex. Daher wurden zusätzlich Aktivitätsdiagramme der Unified Modeling Language (UML) zur Darstellung der Modellierung verwendet [99, 108, 113]. Die nachfolgende Abbildung 1 zeigt ein Minimalbeispiel eines Aktivitätsdiagramms. Es umfasst alle für die Modellierung der Szenarien notwendigen Elemente.

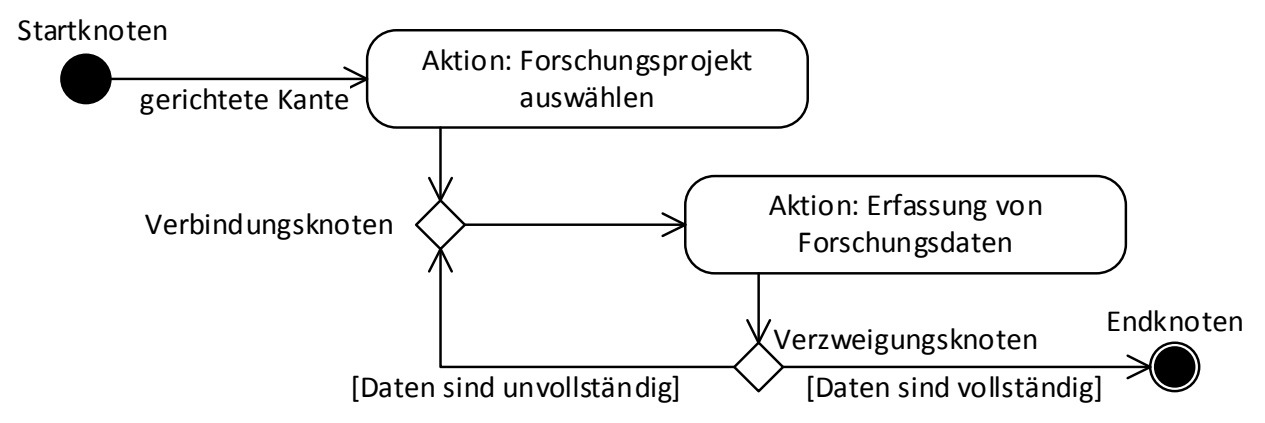

Abbildung 1. Minimalbeispiel eines Aktivitätsdiagramms

\footnotetext{
${ }^{2}$ Die identifizierten Ziele wurden wie folgt nummeriert: Z-X. Z steht für Ziel; X ist die laufende Nummer des Ziels.
} 
Ein Aktivitätsdiagramm beginnt immer mit einem Startknoten und endet mit einem Endknoten. Im Beispiel führt der Startknoten über eine gerichtete Kante zur ersten Aktion. Hier wird zunächst ein Forschungsprojekt ausgewählt. Nach erfolgter Auswahl führt die Aktion weiter zu einem Verbindungsknoten. Dieser vereinigt verschiedene Abläufe zu einer weiteren Aktion, welche die Erfassung von Forschungsdaten repräsentiert. Nach der Aktion folgt ein Verzweigungsknoten. Durch diesen wird geprüft, ob die Forschungsdaten vollständig erfasst wurden. Ist dies nicht der Fall, führt eine gerichtete Kante zum Verbindungsknoten zurück, wodurch eine erneute Erfassung der Forschungsdaten dargestellt wird. Der Ablauf ist beendet, wenn die Forschungsdaten vollständig erfasst wurden. Dies wird durch den Endknoten repräsentiert.

Die Modellierung der Szenarien in Form von Aktivitätsdiagrammen umfasste als ersten Schritt die Identifizierung der Interaktionen des Akteurs mit dem Probandenmanagementsystem, als zweiten Schritt deren Darstellung in einem Aktivitätsdiagramm und im dritten Schritt deren schriftliche Beschreibung. Die Zusammenstellung der Szenarien erfolgte auf Grundlage von Tabelle 2.

\begin{tabular}{l|l} 
Nr. $^{3}$ & Name \\
\hline Nr. & Name des Szenarios \\
Tabelle 3. & Vorlage zur zusammenfassenden Darstellung der identifizierten Szenarien
\end{tabular}

\subsection{Ermittlung der lösungsorientierten Anforderungen}

„Lösungsorientierte Anforderungen werden auf Grundlage von Zielen und Szenarien gewonnen. Sie spezifizieren die geforderten Eigenschaften und Merkmale des zu entwickelnden System[s ...]“ [99, S. 181]. Im Rahmen dieser Dissertation bilden somit die lösungsorientierten Anforderungen das Bindeglied zwischen der ziel- und szenariobasierten Analyse und der Entwicklung des Architekturkonzepts.

Die nachfolgenden Unterabschnitte erörtern im Kontext dieser Arbeit die verschiedenen Arten von lösungsorientierten Anforderungen, die Quellen zu deren Gewinnung sowie das Vorgehen zur Dokumentation der gewonnenen lösungsorientierten Anforderungen.

\subsubsection{Arten von lösungsorientierten Anforderungen}

Auf Grundlage von Pohl et al. [106] wurde zwischen drei Arten von lösungsorientierten Anforderungen unterschieden: funktionale Anforderungen, Qualitätsanforderungen und Randbedingungen. Auf die typische Unterteilung zwischen funktionalen und nicht-funktionalen Anforderungen [114] wurde verzichtet, da nach Pohl [99] nicht-funktionale Anforderungen entweder

\footnotetext{
${ }^{3}$ Die identifizierten Szenarien wurden wie folgt nummeriert: S-X. S steht für Szenario; X ist die laufende Nummer des Szenarios.
} 
unterspezifizierte funktionale Anforderungen oder Qualitätsanforderungen sind. Die drei Arten von lösungsorientierten Anforderungen sind wie folgt definiert:

Funktionale Anforderungen: „Eine funktionale Anforderung ist eine Anforderung bezüglich [...] eines Verhaltens, das von einer Funktion des Systems bereitgestellt werden soll.“ [106, S. 16]

Qualitätsanforderungen: „Eine Qualitätsanforderung ist eine Anforderung, die sich auf ein Qualitätsmerkmal bezieht, das nicht durch funktionale Anforderungen abgedeckt wird.“ [106, S. 16]

Randbedingung: „Eine Randbedingung ist eine Anforderung, die den Lösungsraum jenseits dessen einschränkt, was notwendig ist, um die funktionalen Anforderungen und die Qualitätsanforderungen zu erfüllen.“ [106, S. 17]

Basierend auf diesen Definitionen wurde jede identifizierte Anforderung einer der drei Arten von lösungsorientierten Anforderungen zugeordnet. Sie bilden die Grundlage zur Modellierung des Architekturkonzepts zu sowie der prototypischen Implementierung [99, 103, 108].

\subsubsection{Quellen zur Gewinnung von lösungsorientierten Anforderungen}

Die primäre Quelle zur Gewinnung der lösungsorientierten Anforderungen waren die Stakeholder, welche durch die identifizierten Ziele und Szenarien für ein Probandenmanagementsystem auf Basis der Interviews repräsentiert werden [102, 103]. Im ersten Schritt wurden die paraphrasierten Aussagen der Stakeholderinterviews einzeln dahingehend geprüft, ob sich aus der Aussage eine Anforderung ergibt. War dies der Fall, wurde die Anforderung einer der drei Arten von lösungsorientierten Anforderungen zugeordnet, sofern noch keine bedeutungsgleiche Anforderung existierte. Der zweite Schritt umfasste die Analyse der Ziele im Hinblick auf die Identifizierung neuer Anforderungen. Dabei entsprach das Vorgehen der Analyse dem der qualitativen Inhaltsanalyse (siehe Abschnitt 2.1.2). Im letzten Schritt wurden die Szenarien analysiert. Die Analyse erfolgt im Hinblick auf die Erfüllung der Szenarien, d. h., welche Anforderungen notwendig waren um die Szenarien vollständig zu realisieren.

Neben den Stakeholdern können auch vorhandene Dokumente eine wichtige Quelle für lösungsorientierte Anforderungen sein [106]. Dies sind beispielsweise Standards, Publikationen oder Prozessbeschreibungen [99]. Zur Durchsicht der Dokumente empfiehlt Pohl [99] die Methodik des perspektivenbasierten Lesens: Der Leser nimmt dabei eine zuvor definierte Perspektive ein und blendet beim Lesen alle davon abweichenden Details des Dokuments aus. Im Rahmen dieser Dissertation war die Perspektive definiert als das Probandenmanagementsystem an der Universitätsmedizin Göttingen. Konkret bedeutete dies die „effiziente und einheitliche Verwaltung sowie die langfristige Nachverfolgung von Probanden über mehrere medizinische Forschungsprojekte“. 
Zur Identifizierung relevanter Dokumente wurde zunächst eine Literaturrecherche durchgeführt (siehe Anhang A.1). Ausgangspunkt der Literaturrecherche bildeten alle Publikationen, die im Forschungsumfeld dieser Dissertation veröffentlicht wurden [1, 7, 14, 109, 115-118].

\subsubsection{Dokumentation der lösungsorientierten Anforderungen}

Die Dokumentation der gewonnenen lösungsorientierten Anforderungen erfolgte schriftlich unter Verwendung einer Vorlage (siehe Tabelle 4). Die Vorlage in Tabelle 4 zur strukturierten Beschreibung der Anforderungen umfasst eine Nummer, eine kurze Beschreibung sowie einen Verweis auf die Quelle der Anforderung. Beim Verweis auf die Quelle gilt immer, dass der Verweis auf ein Szenario auch dessen Ziele beinhaltet bzw. die den Zielen zugrunde liegenden Interviews.

\begin{tabular}{l|l|l} 
Nr. $^{4}$ & Beschreibung & Quelle \\
\hline Nr. & Kurze Beschreibung der Anforderung & $\begin{array}{l}\text { Quelle der An- } \\
\text { forderung }\end{array}$
\end{tabular}

Tabelle 4. Vorlage zur strukturierten Beschreibung der lösungsorientierten Anforderungen

Innerhalb des Dokumentationsprozesses der Anforderungen können zusätzliche Kategorien zum Zwecke einer besseren Strukturierung gebildet werden. Eine Kategorie ist immer einer der drei Arten von Anforderungen (funktionale Anforderungen, Qualitätsanforderungen oder Randbedingungen) zugeordnet und gruppiert ähnliche Anforderungen. Die Gruppierung in Kategorien ermöglicht einen besseren Überblick und wird nur bei Bedarf erstellt.

\subsection{Entwicklung des Architekturkonzepts}

Der erste Teilaspekt der zweiten Forschungsfragestellung umfasst die Entwicklung eines Architekturkonzepts basierend auf den identifizierten Szenarien und Schnittstellen. Das zu entwickelnde Architekturkonzept ist eine detaillierte Beschreibung der Softwarearchitektur eines Informationssystems. Gharbi et al. [119, S. 21] definieren die Softwarearchitektur als „,...] die grundlegenden Prinzipien und Regeln für die Organisation eines System[s] sowie dessen Strukturierung in Bausteine und Schnittstellen [Bausteinschnittstellen] und deren Beziehungen zueinander wie auch zur Umgebung [...]“.

Ein Baustein ist das zentrale Element der Softwarearchitektur und repräsentiert beispielsweise Funktionen, Klassen, verwendete Softwarebibliotheken oder die Datenbank [120]. Eine Bausteinschnittstelle beschreibt einen wohldefinierten Zugangspunkt zum System oder zu dessen Bausteinen [119]. Bausteine bieten zum einen eigene Bausteinschnittstellen an, zum anderen

\footnotetext{
${ }^{4}$ Die identifizierten Anforderungen wurden wie folgt nummeriert: A-X-Y. A steht für Anforderung; X kann den Wert F für funktionale Anforderung, Q für Qualitätsanforderung oder R für Randbedingung annehmen; Y ist die laufende Nummer je Anforderungsart.
} 
konsumieren sie andere Bausteinschnittstellen und kapseln so die eigentliche Implementierung [119].

Der Entwicklungsprozess des Architekturkonzepts umfasst die Abbildung der lösungsorientierten Anforderungen auf die einzelnen Bausteine der Softwarearchitektur [120]. Dabei repräsentieren die lösungsorientierten Anforderungen die Szenarien sowie die Schnittstellen zu externen Systemen, wie sie in der zweiten Forschungsfragestellung gefordert werden. Jede Anforderung wird dahingehend analysiert, welche Bausteine (Funktionen, Klassen etc.) sich aus ihr ergeben. Die Abbildung von Anforderungen auf die einzelnen Bausteine wird nachfolgend als Fachdomäne bezeichnet. Folglich bildet die Fachdomäne den Kern des Architekturkonzepts.

\subsubsection{Domain-Driven Design}

Die Modellierung der Fachdomäne erfolgte durch das von Eric Evans [121] eingeführte Konzept des Domain-Driven Design. Im Vordergrund steht dabei nicht die Entwicklung des Systems, sondern die Lösung der Problemstellung unter Berücksichtigung der Domänenexperten (Stakeholder) [121]. Die Anwendung des Domain-Driven Design zur Entwicklung des Architekturkonzepts bot sich an, da durch die identifizierten Ziele, Szenarien und lösungsorientierten Anforderungen die Problemstellung bereits umfassend analysiert wurde [99]. Zudem bietet das Domain-Driven Design eine strukturierte und nachvollziehbare Vorgehensweise zur Modellierung der Fachdomäne.

\subsubsection{Architekturstil Ports and Adapters}

Ein wichtiger Aspekt beim Entwurf einer Softwarearchitektur unter Anwendung von DomainDriven Design ist die Isolierung der Fachdomäne [121]. Die Isolierung soll die Unabhängigkeit der Fachdomäne von der eigentlichen Implementierung der Softwareanwendung sicherstellen. Evans [121] empfiehlt die Unterteilung der Softwarearchitektur in vier Schichten: Präsentation, Applikationsschicht, Fachdomäne und technische Infrastruktur (z. B. Persistenz, Sicherheit und Kommunikation). Die geforderte Schichtentrennung wird meist in der Praxis nicht vollständig eingehalten, sodass eine Überlappung der Schichten entsteht [120]. Hierdurch verringert sich die Verständlichkeit der Softwarearchitektur [120].

Vernon [122] und Starke [120] empfehlen daher die Verwendung des Architekturstils Ports and Adapters von Alistair Cockburn [123] zur Strukturierung der Fachdomäne (siehe Abbildung 2). Die Fachdomäne wird innerhalb des Architekturstils durch primäre und sekundäre Schnittstellen, sogenannte Ports, gekapselt [124]. Ziel der Kapselung ist die vollständige Isolierung der Fachdomäne von der eigentlichen Umsetzung in Form der Benutzeroberfläche oder technischen Infrastruktur wie der Datenbank. 


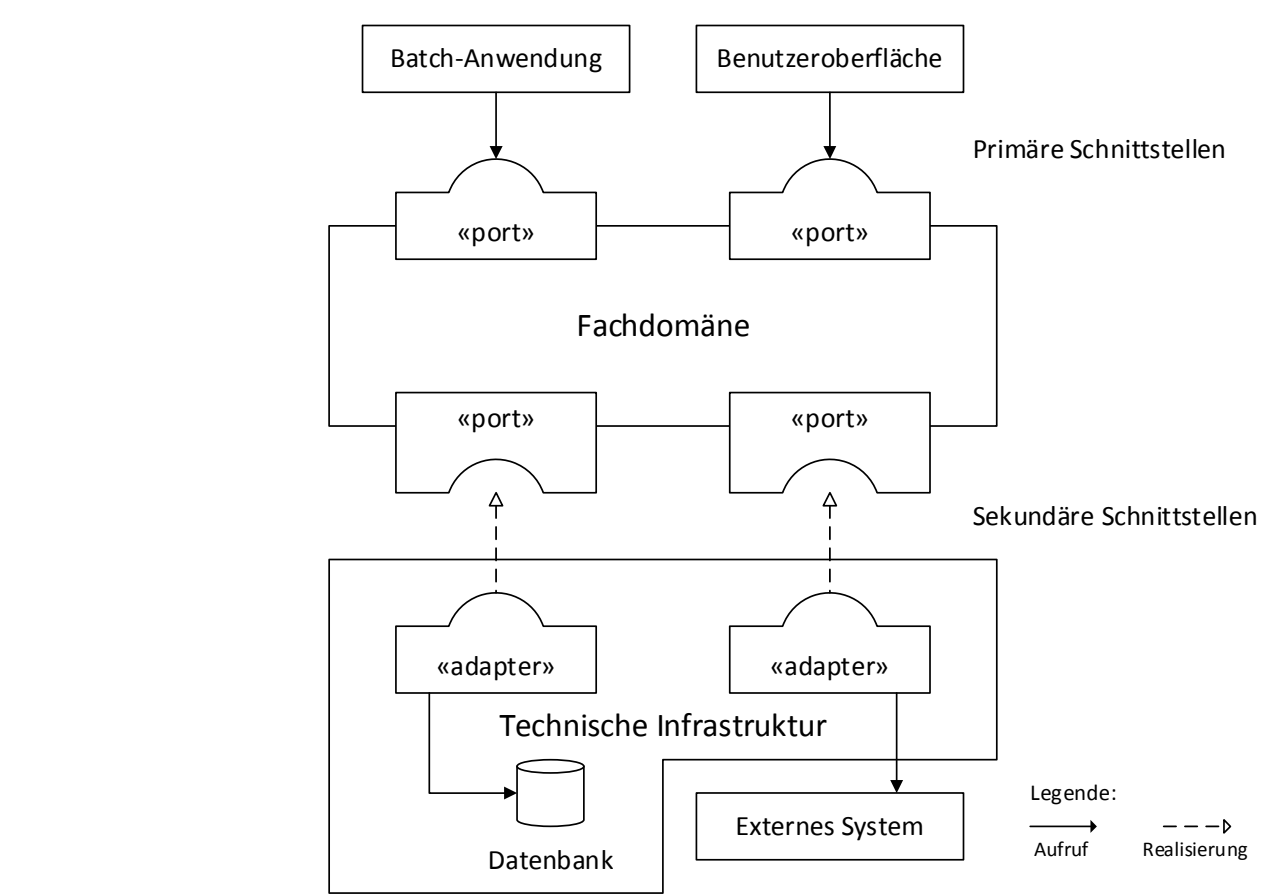

Abbildung 2. Architekturstil „Ports and Adapters“ [120]

Primäre Schnittstellen werden von der Fachdomäne zur direkten Verwendung bereitgestellt. Sie bilden beispielsweise die Grundlage zur Implementierung der Benutzeroberfläche. Sekundäre Schnittstellen müssen durch geeignete Adapter implementiert werden, z. B. für den Zugriff auf die Datenbank. Die Implementierung durch die Adapter wird zur Laufzeit der Softwareanwendung durch geeignete Mechanismen, wie beispielsweise „Dependency Injection“, bereitgestellt [120, 125, 126].

\subsubsection{Modellierung der Fachdomäne}

Nach Evans [121] ist die Fachdomäne innerhalb des Domain-Driven Design aus folgenden Bausteinen zusammengesetzt:

Entities bilden die Kernobjekte der Fachdomäne und werden direkt aus den lösungsorientierten Anforderungen gewonnen. Darüber hinaus verfügen sie über eine eindeutige Identität und einen unveränderlichen Lebenszyklus [120, 121].

Value Objects sind meist kleine Objekte (z. B. ein Datum) und besitzen keine eigene Identität. Sie werden im Zusammenhang mit Entities oder anderen Value Objects verwendet, um deren Zustand zu beschreiben. Ebenso wie Entities ergeben sie sich aus den lösungsorientierten Anforderungen [120, 121, 127].

Aggregates beinhalten miteinander assoziierte Entities oder Value Objects. Sie basieren immer auf einer Entity und kapseln diese und die assoziierten Objekte gegenüber der Außenwelt ab $[120,121]$. 
Services bieten im Sinne des Architekturstils Ports and Adapters primäre Schnittstellen an, welche die Abläufe in der Fachdomäne beschreiben. Diese Abläufe korrespondieren mit den identifizierten Szenarien [120, 121].

Factories dienen der Erzeugung von komplexen Entities und Aggregates. Eine Verwendung von Factories ist nicht zwingend erforderlich [120, 121].

Repositories bieten Funktionen zur Speicherung und Suche von Entities und Aggregates. Im Sinne des Ports and Adapters Architekturstils sind sie sekundäre Schnittstellen, die durch geeignete Adapter implementiert werden müssen [120, 121].

Entities, Value Objects und Aggregates werden im Nachfolgenden als Domänenmodell bezeichnet, um sie von den primären und sekundären Schnittstellen abzugrenzen. Die sekundären Schnittstellen umfassen neben Repositories auch mögliche (reale) Schnittstellen zu externen Systemen, die sich aus den Anforderungen ergeben oder von Services benötigt werden. Auf eine Modellierung der Repositories wurde verzichtet, da diese bereits durch die im nachfolgenden Abschnitt beschriebene Softwarebibliothek Spring bereitgestellt werden. Ebenso wurde bei der Modellierung versucht, komplexe Entities und Aggregates zu vermeiden, sodass die Verwendung von Factories nicht notwendig war.

Die Darstellung der Fachdomäne erfolgte unter Verwendung des UML Klassendiagramms. Es ermöglicht, die Eigenschaften und die Beziehungen der Fachdomäne umfassend darzustellen [113]. Um eine mögliche internationale Nutzbarkeit und Weiterentwicklung zu gewährleisten, erfolgten die Modellierung und die damit verbundene prototypische Implementierung in englischer Sprache.

Abbildung 3 umfasst ein Minimalbeispiel für ein Domänenmodell mit Entities, Value Objects und Aggregates.

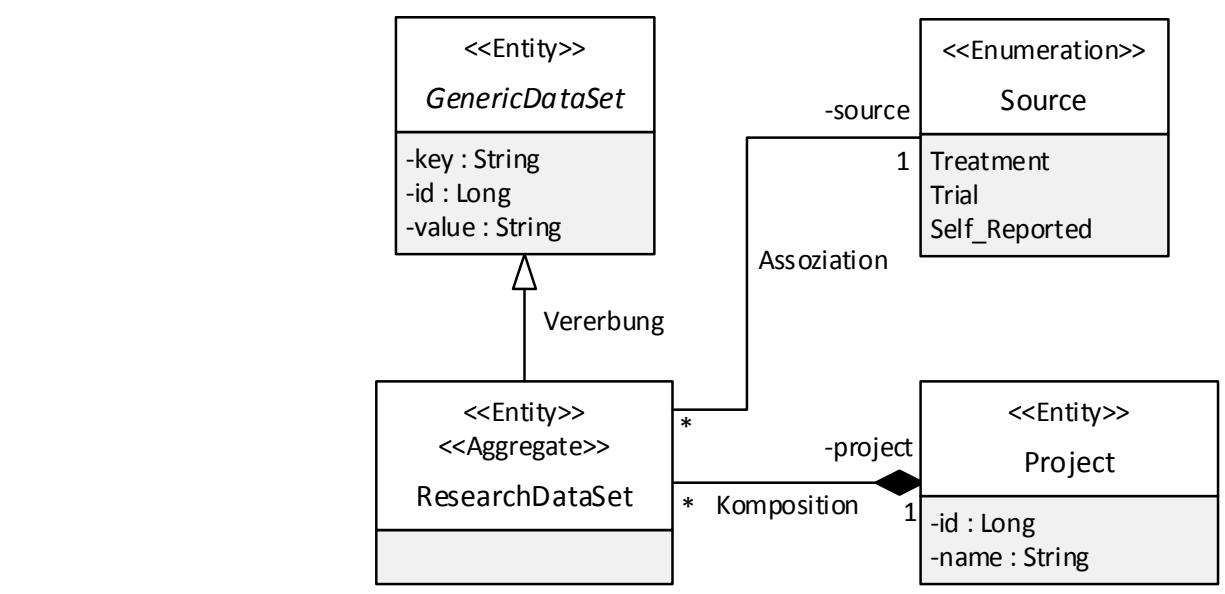

Abbildung 3. Minimalbeispiel eines Klassendiagramms zur Modellierung des Domänenmodells

Das Klassendiagramm umfasst alle notwendigen Elemente zur Modellierung des Domänenmodells. Im Klassendiagramm werden nur die privaten Eigenschaften bzw. Attribute der Klassen 
dargestellt; auf die Modellierung von öffentlichen Zugriffsmethoden wird zur besseren Übersichtlichkeit verzichtet. Das in Abbildung 3 dargestellte Domänenmodell umfasst die notwendigen Entities, Value Objects und Aggregates zur Umsetzung des Beispielszenarios aus Abbildung 1.

Die Klasse ResearchDataSet (Forschungsdatensatz) erbt alle Eigenschaften der übergeordneten Klasse GenericDataSet und erweitert diese Eigenschaften um eine Beziehung zu einem Project (Forschungsprojekt). Das ResearchDataSet steht in einer engen Beziehung zu einem Project; dies wird durch eine Komposition dargestellt. Komposition bedeutet, dass ein ResearchDataSet nicht ohne ein Project existieren kann. Ein ResearchDataSet verfügt zusätzlich über eine Beziehung zur Klasse Source (Quelle). Die Beziehung wird in Form einer einfachen Assoziation dargestellt, da beide Klassen unabhängig voneinander existieren können.

Die Klassen GenericDataSet, ResearchDataSet sowie Project sind im Sinne des Domain-Driven Design Entities, da alle eine eigene Identität besitzen. Im Diagramm wird dies durch den Stereotyp „Entity“ dargestellt. Die Klasse Source hingegen ist ein Value Object, da es im aktuellen Beispiel keine eigene Identität besitzt, sondern eine Aufzählung („Enumeration“) ist. Das Laden und Speichern der Klasse Source erfolgt über die assoziierte Klasse ResearchDataSet, daher ist diese mit dem Stereotyp „Aggregate“ versehen. Abbildung 4 greift das Beispiel auf und umfasst die notwendigen primären und sekundären Schnittstellen für das Domänenmodell.

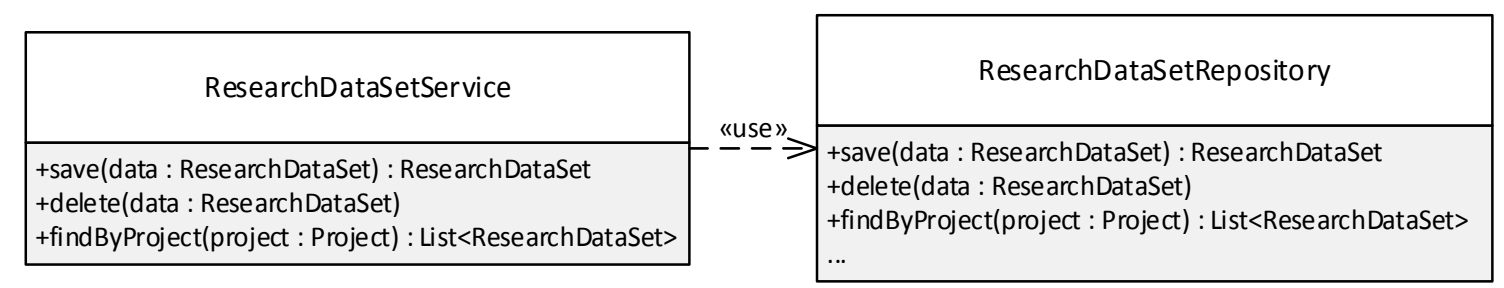

Abbildung 4. Minimalbeispiel eines Klassendiagramms zur Modellierung der primären und sekundären Schnittstellen

ResearchDataSetService bildet die primäre Schnittstelle und umfasst Methoden zur Speicherung, Suche und Löschung von ResearchDataSets. Wie zuvor erwähnt, wurde auf eine umfassende Modellierung der Repositories verzichtet, da diese automatisch bereitgestellt werden. Daher sind in Abbildung 4 die Methoden von ResearchDataSetRepositories nur angedeutet. ResearchDataSetService leitet die Anfragen, beispielsweise zur Suche, an das jeweilige Repository weiter; dies wird durch einen gestrichelten Pfeil mit der Beschriftung «use» dargestellt.

\subsection{Prototypische Implementierung des Architekturkonzepts}

Ziel der prototypischen Implementierung war die Erfüllung des zweiten Teilaspekts der zweiten Forschungsfragestellung. Dieser Teilaspekt umfasst die Umsetzung des Architekturkonzepts und somit die Prüfung der Realisierbarkeit des Architekturkonzepts [114].

Darüber hinaus bildete der Prototyp die Grundlage zur Validierung des Architekturkonzepts durch die Stakeholder. Der Prototyp sollte daher in Form eines Pilotsystems realisiert werden, 
sodass sowohl die Funktionalität als auch die Qualität für den produktiven Einsatz des Probandenmanagementsystems entsprechend hoch ist [128].

Die Implementierung des Prototyps erfolgte in der Programmiersprache Java [129]. Diese wurde gewählt, da prominente Softwareanwendungen im Bereich der medizinischen Forschung ebenfalls diese Programmiersprache nutzen [130-134]. Ebenso bietet die Java-Umgebung umfangreiche und in der Industrie erprobte Softwarebibliotheken zur Entwicklung von Informationssystemen [135].

\subsubsection{Aufbau und Strukturierung des Prototyps}

Die dem Prototyp zugrundeliegende Softwarearchitektur basiert auf dem Architekturstil „Ports and Adapters“. Dieser unterteilt ein System in unterschiedliche Komponenten, die Abhängigkeiten zueinander aufweisen. Zur Darstellung der einzelnen Komponenten des Prototyps und deren Beziehungen wurde das UML Komponentendiagramm verwendet [113].

Abbildung 5 zeigt basierend auf den Bereichen des Domain-Driven Design ein Minimalbeispiel des Komponentendiagramms. Alle Komponenten sind Teil der übergeordneten Komponente Softwarearchitektur. Sowohl primäre als auch sekundäre Schnittstellen besitzen eine Verwendungsbeziehung zum Domänenmodell. Primäre Schnittstellen delegieren Aufrufe zu den sekundären Schnittstellen; dies ist ebenfalls durch eine Verwendungsbeziehung dargestellt.

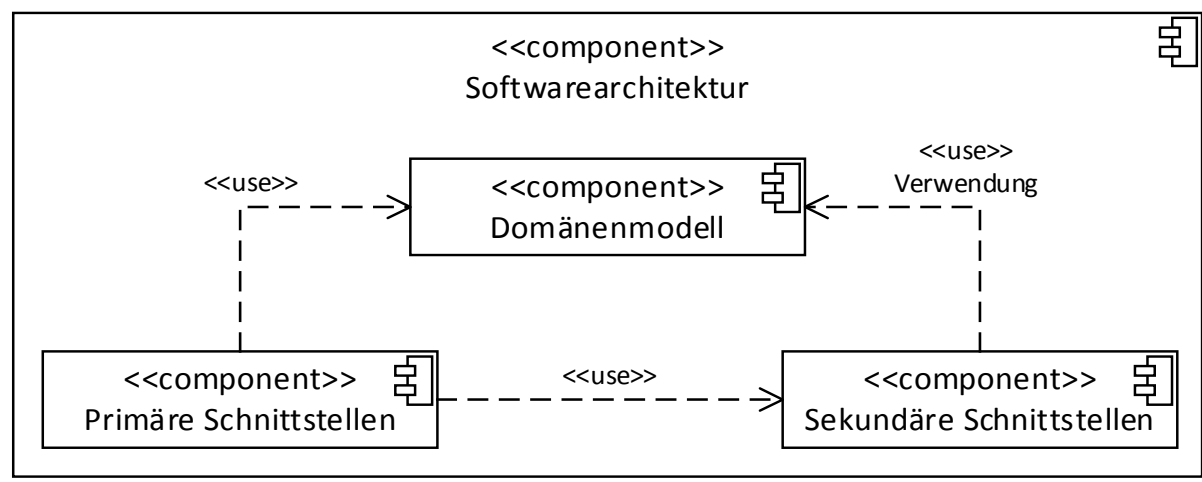

Abbildung 5. Minimalbeispiel eines Komponentendiagramms zur Beschreibung des Aufbaus und der Struktur des Prototyps

Das Komponentendiagramm bietet nur eine Möglichkeit zur Visualisierung der Abhängigkeiten, nicht aber zu deren konkreten Umsetzung. Im Java-Umfeld wird zur Umsetzung der Abhängigkeiten das Softwarewerkzeug Maven ${ }^{5}$ verwendet [136]. Maven ermöglicht die Verwaltung der Komponenten und deren Abhängigkeiten untereinander sowie die Abhängigkeiten zu externen Softwarebibliotheken. Darüber hinaus wird es zur automatisierten Erstellung und Testung von Softwareanwendungen verwendet. Somit ist Maven ein wichtiges Werkzeug zur Erstellung qualitätsgesicherter und valider Softwareanwendungen [136]. 


\subsubsection{Abbildung der Fachdomäne}

Eine Anforderung des Architekturstils „Ports and Adapters“ ist die Bereitstellung von konkreten Implementierungen der sekundären Schnittstellen zur Laufzeit [121]. Cockburn [123] und Vernon [122] empfehlen die Verwendung der Softwarebibliothek Spring ${ }^{6}$. Spring ist eine in der Industrie erprobte und verbreitete Softwarebibliothek zur Entwicklung von (webbasierten) Systemen $[137,138]$. Sie bietet unter anderem die Möglichkeit eine spezifische Implementierung zur Laufzeit des Systems bereitzustellen. Zusätzlich verfügt Spring über verschiedene Module, beispielsweise zur Authentifizierung und Autorisierung, Testung, aspektorientierten Programmierung sowie zur Abstraktion des Datenbankzugriffs durch Repositories [137, 138].

Im Rahmen der prototypischen Implementierung sollte eine funktionsfähige und nutzbare Anwendung entstehen. Dies erforderte zum einen die Implementierung des Domänenmodells und zum anderen dessen Abbildung in einer relationalen Datenbank [139].

Im Bereich der Softwareentwicklung wird die Speicherung von Objekten in einer relationalen Datenbank als objektrelationale Abbildung bezeichnet [140]. Java und Spring bieten geeignete Softwarebibliotheken, um diese Abbildung zu vereinfachen [138]. Die Abbildung erfolgte unter Verwendung der Softwarebibliothek Hibernate 7 . Hibernate wird vollständig von Spring unterstützt und verfügt zusätzlich über ein Modul zur Nachverfolgung von Änderungen an Datensätzen [140]. Durch die Verwendung von Spring und Hibernate wurden die im Domain-Driven Design geforderten Repositories zur Verfügung gestellt [138].

\subsubsection{Entwicklung der Benutzeroberfläche}

Ein weiterer elementarer Bestandteil des Softwareprototyps ist eine webbasierte Benutzeroberfläche. Zur Implementierung der Benutzeroberfläche wurde die Softwarebibliothek Vaadin ${ }^{8}$ verwendet. Vaadin ermöglicht die einfache Erstellung von komplexen Webanwendungen mit Hilfe der Programmiersprache Java [141]. Dabei wird die eigentliche Webanwendung von der Implementierung vollständig abstrahiert, sodass keine zusätzlichen Kenntnisse, wie beispielsweise HTML oder JavaScript, notwendig sind. Die Implementierung erfolgt vollständig in Java und ist vergleichbar mit der Entwicklung einer Desktopanwendung. Vaadin integriert sich ebenfalls in die Spring-Umgebung, sodass innerhalb des Prototyps ein einheitliches Programmiermodell sichergestellt ist [142].

\subsection{Vorgehen zur Validierung}

Der Begriff „Validierung“ wurde im Bereich der Softwareentwicklung maßgeblich durch Barry W. Boehm geprägt [99, 128]. Boehm [143] koppelte den Begriff mit der Fragestellung, ob das

\footnotetext{
${ }^{6}$ http://www.spring.io

${ }^{7}$ http://www.hibernate.org

${ }^{8}$ http://www.vaadin.com
} 
richtige Produkt bzw. System entwickelt wird: „Am I building the right product?“ Hierdurch soll sichergestellt werden, dass das System im Sinne der Stakeholder entwickelt wird [128].

Es ist folglich das Ziel der Validierung zu untersuchen, ob das Probandenmanagementsystem den Erwartungen der Stakeholder entspricht. Die Grundlage zur Durchführung der Validierung bildete die im vorangegangenen Abschnitt durchgeführte prototypische Implementierung. Diese wurde im ersten Schritt (siehe Abschnitt 2.5.1) im Hinblick auf die Erfüllung der lösungsorientierten Anforderungen untersucht. In diesem Zusammenhang wurde geprüft, welche Ziele und Szenarien durch den Prototyp abgebildet werden. Im zweiten Schritt der Validierung (Abschnitt 2.5.2) erfolgt die Bewertung der Nutzbarkeit des prototypisch entwickelten Probandenmanagementsystems durch einen Usability Test.

\subsubsection{Funktionsprüfung}

Die Funktionsprüfung sollte untersuchen, welche lösungsorientierten Anforderungen im Prototyp umgesetzt wurden. Innerhalb dieser Dissertation wurde zwischen drei Arten von Anforderungen unterschieden: funktionale Anforderungen, Qualitätsanforderungen sowie Randbedingungen. Die Erfüllung der beiden letzteren Anforderungsarten konnten nicht durch eine Funktionsprüfung abgebildet werden, da für deren Erfüllung beispielsweise eine hohe Nutzerzufriedenheit oder die Einführung von organisatorischen Maßnahmen erforderlich ist. Daher stand die Erfüllung der funktionalen Anforderungen im Vordergrund der Funktionsprüfung. Die Erfüllung einer Anforderung durch den Prototyp wurde zum einen beschrieben und zum anderen durch einen Screenshot belegt.

Neben den Anforderungen wurden auch Ziele und Szenarien identifiziert, die wiederum die Grundlage für die funktionalen Anforderungen bildeten (siehe Abschnitt 2.2). Daher wurde zum Abschluss der Funktionsprüfung dargestellt, welche der zugehörigen Ziele und Szenarien durch den Prototyp des Probandenmanagementsystems erfüllt wurden. Hierfür wurde die nachfolgende Vorlage in Tabelle 5 verwendet, die den Zusammenhang zwischen Zielen, Szenarien und funktionalen Anforderungen sowie deren Erfüllung mit einem Beispiel darstellt. Ein Szenario kann nur erfüllt werden, wenn alle zugehörigen Ziele erfüllt sind.

\begin{tabular}{l|l|l|l} 
Ziel & Szenario & Anforderung & Erfüllt \\
\hline Z-1 $(\checkmark)$ & & A-F-1 $(\checkmark)$ & \\
Z-2 $(\checkmark)$ & S-1 $(\checkmark)$ & A-F-2 $(\checkmark)$ & Ja \\
\hline Z-3 (x) & & A-F-3 $(\checkmark)$ & \\
Z-4 ( $)$ & S-2 (x) & A-F-2 $(\checkmark)$ & Nein
\end{tabular}

Tabelle 5. Vorlage zur Dokumentation des Erfüllungsgrades der Ziele und Szenarien

\subsubsection{Usability Test}

Innerhalb der Validierung sollte der Prototyp des Probandenmanagementsystems durch die Stakeholder genutzt und erprobt werden. Da ein freies Experimentieren der Stakeholder mit dem 
System mitunter zu extrem subjektiven Resultaten führen kann [99], wurde ein Validierungsprotokoll in Form eines Usability-Tests auf Grundlage der identifizierten Szenarien entwickelt. Durch die Verwendung des Usability-Tests sollten subjektive Eindrücke reduziert werden, sodass der Fokus des Usability-Tests auf den Szenarien lag.

\section{Entwicklung des Usability-Tests}

Ein Usability-Test (Benutzerakzeptanztest) basiert grundlegend auf den Zielen der Stakeholder. Im Rahmen des Tests bearbeiten die Stakeholder mit dem System einzelne Szenarien, die wiederum zur Erfüllung der Ziele führen [144]. Die benötigten Szenarien für den Usability-Test werden aus den identifizierten Szenarien der ziel- und szenariobasierten Analyse abgeleitet. Im Vordergrund steht dabei, dass die Szenarien typische Arbeitsabläufe normaler Systembenutzer wiedergeben. Tätigkeiten eines Systemadministrators werden daher nicht betrachtet.

Kern des Usability-Tests bilden fünf Szenarien (siehe Anhang A.3, Abschnitt Szenario 1 bis 5). Innerhalb des ersten Szenarios werden die Stakeholder mit der Probandenübersicht vertraut gemacht. Diese bietet den Stakeholdern eine Liste aller ihnen zugeordneten Probanden sowie die Forschungsprojekte, an denen die Probanden teilnehmen. Im zweiten Szenario wird eine kleinere Probandenübersicht genutzt, um einen bereits im System erfassten Probanden einem neuen Forschungsprojekt zuzuordnen. Da auch Probanden neu im System erfasst werden müssen, wird im dritten Szenario ein Proband mit seinen Stammdaten neu angelegt. Durch die interne Duplikaterkennung stellt das System fest, dass dieser Proband bereits im System existiert, sodass kein neuer Proband angelegt, sondern auf den vorhandenen Probanden verwiesen wird. Gegenstand des vierten Szenarios ist die Anbindung an das Krankenhausinformationssystem zum Abruf von Probandenstammdaten. Durch die Eingabe der Patientenkennung werden die Stammdaten des Probanden aus dem Krankenhausinformationssystem abgerufen und im System gespeichert. Das fünfte Szenario umfasst die Kommunikation mit einem externen System zur Pseudonymisierung eines Probanden. In Verbundforschungsprojekten ist dies ein typisches Szenario [47]. Die Stammdaten des Probanden werden über die Schnittstelle des externen Systems übertragen. Alle Szenarien wurden zu Testzwecken durch drei Personen mit dem Prototyp des Probandenmanagementsystems bearbeitet, dabei kam es zu keinen Beanstandungen. Daher musste keine Änderung an den Szenarien vorgenommen werden.

Direkt nach der Bearbeitung eines Szenarios wurden die Stakeholder gebeten eine Bewertung des Szenarios vorzunehmen (siehe Anhang A.3, Abschnitt Szenario 1). Durch die Szenariobewertung sollte die Nützlichkeit der zugrundeliegenden Systemfunktion ermittelt werden. Die Bewertung umfasste eine Likert-Skala mit fünf Antwortmöglichkeiten sowie eine Freitextantwort [111, 144].

Nach erfolgter Bearbeitung aller Szenarien erfolgte eine zusammenfassende Bewertung des gesamten Systems. Diese Bewertung repräsentierte die Benutzerakzeptanz, d. h. die Stakeholder bewerteten, ob der Prototyp ihre Erwartungen an ein Probandenmanagementsystem erfüllte. Hierfür wurde ein standardisierter Test, der System Usability Scale (SUS), verwendet [144]. 
Der System Usability Scale bietet eine schnelle Möglichkeit um die Nutzbarkeit eines Systems zu erfassen [145]. Er umfasst zehn Fragen, die abwechselnd positiv und negativ formuliert sind. Die Beantwortung der Fragen erfolgt durch eine Likert-Skala mit fünf Antwortmöglichkeiten (siehe Anhang A.3, Abschnitt Systembewertung). Ursprünglich wurde der System Usability Scale in englischer Sprache entwickelt [145], er ist jedoch auch in Deutsch verfügbar [146], daher wurde die deutsche Version des SUS verwendet. Zum Abschluss des Usability-Tests wurden die Stakeholder gebeten, fehlende Systemfunktionen zu nennen, die sie für die tägliche Arbeit mit dem System benötigen würden.

Ein großer Nachteil des Usability-Tests ist, dass er in einer definierten Umgebung unter vorgegebenen Testbedingungen erfolgt. Hierdurch kann die Nutzbarkeit des Probandenmanagementsystems im produktiven Einsatz nicht ermittelt werden. Um dennoch einen ersten Eindruck über die Nutzbarkeit des Systems im produktiven Umfeld zu erhalten, wurde die bereits angeführte Vorversion des Probandenmanagementsystems in den Usability-Test einbezogen [109].

Die Vorversion des Probandenmanagementsystems bildete die Grundlage dieser Dissertation und ist derzeit in drei Forschungsprojekten an der Universitätsmedizin Göttingen im Einsatz. Sie verfolgte eine ähnliche Zielsetzung wie der zu entwickelnde Prototyp, verfügt jedoch nicht über eine projektübergreifende Verwaltung von Probanden [109]. Nutzte ein Stakeholder bereits die Vorversion, erhielt dieser eine Vorabbefragung vor dem eigentlichen Usability-Test. In der Vorabbefragung sollte der Stakeholder die Vorversion des Probandenmanagementsystems bewerten. Hierfür wurde eine abgeänderte Form des System Usability Scale genutzt, in der die einzelnen Fragen in ihre Präsensform umgewandelt wurden (siehe Anhang A.3, Allgemeine Angaben). Zusätzlich enthielt die Vorabbefragung für alle Stakeholder jeweils eine Frage zur Person, zur Rolle sowie zum aktuellen Umgang mit Probanden.

\section{Durchführung der Validierung}

Die Durchführung eines Usability-Tests wird mit ca. zehn Teilnehmern empfohlen [147-149]. Hierdurch kann sichergestellt werden, dass ein Großteil der Probleme einer Software erkannt wird. Eine erste Einschätzung zur Nutzbarkeit eines Systems ist durchaus auch mit fünf Personen möglich [148].

Ziel der Validierung ist es zu prüfen, ob die Erwartungen der Stakeholder an den Prototyp erfüllt werden. Daher wurden der Usability-Test mit insgesamt zehn Stakeholdern durchgeführt: vier Ärzten, zwei wissenschaftlichen Mitarbeitern und vier Studienassistenten. Deren Auswahl erfolgte auf Grundlage der bereits durchgeführten Stakeholderinterviews (siehe Abschnitt 2.1.1).

Ein weiteres Kriterium war, dass alle teilnehmenden Stakeholder potentielle Nutzer des Systems sein sollen. Da dieses Kriterium nicht von allen 17 Personen im Rahmen der Stakeholderinterviews erfüllt wurde, beispielsweise dem Datenschutzbeauftragten, wurden zusätzliche sechs Stakeholder aus der Universitätsmedizin Göttingen in die Usability-Tests einbezogen. 
Dies war notwendig, da sonst zu wenig Stakeholder an der Validierung teilgenommen hätten. Fünf der zehn Stakeholder verwendeten zudem bereits die angeführte Vorversion des Probandenmanagementsystems, sodass eine erste Einschätzung zur Nutzbarkeit des Systems in realen Forschungsprojekten möglich war [148].

\section{Auswertung der Testergebnisse}

Die Auswertung der Usability-Tests ist unterteilt in die drei Bereiche Freitext, Szenariobewertung und Systembewertung mittels System Usability Scale. Zur Auswertung des Freitextes wurde die bereits angeführte qualitative Inhaltsanalyse verwendet (siehe Abschnitt 2.1.2). Die qualitative Inhaltsanalyse konnte angewendet werden, da Freitextantworten unter einer zentralen Fragestellung stehen. So war sichergestellt, dass nur relevante Aspekte in die Auswertung aufgenommen wurden.

Grundlage der Szenariobewertung war eine Likert-Skala; für die Auswertung wurden die absoluten Häufigkeiten der fünf Antwortmöglichkeiten verwendet [150]. Prinzipiell wäre eine statistische Auswertung ebenfalls möglich gewesen; auf diese wurde jedoch verzichtet, da die Bewertung nur durch zehn Stakeholder erfolgte und so die Fallzahl gering war.

Die umfassendste Auswertung erfolgte für die beiden Systembewertungen mittels System Usability Scale. Dieser umfasste zehn Fragen, auf deren Grundlage der sogenannte SUS $_{\text {Score }}$ berechnet wird. Dieser hat einen Wertebereich von 0 bis 100 und gibt die Usability des Systems

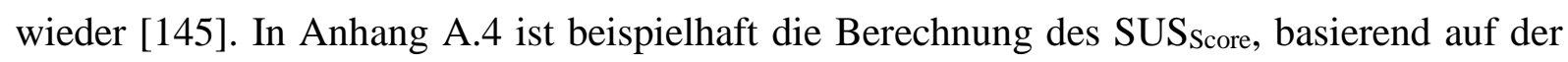
Originalarbeit von Brooke [145], beschrieben.

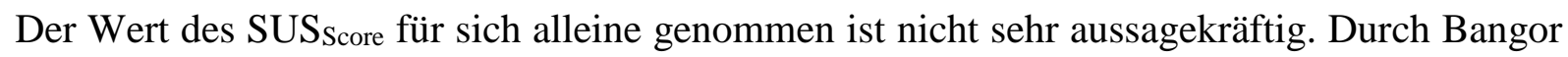
et al. [151] wurde im Rahmen einer empirischen Analyse des System Usability Scale eine Be-

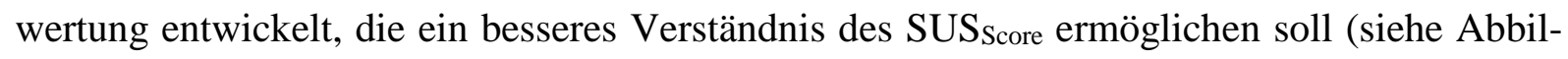
dung 6).

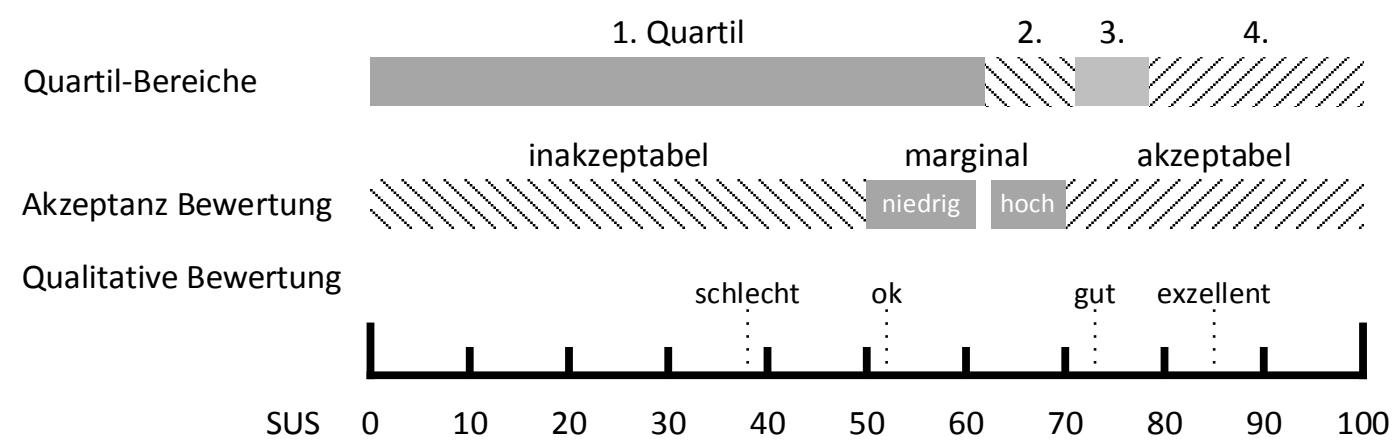

Abbildung 6. Bewertung des SUSscore nach Bangor et al. [151]

Durch die von Bangor et al. [151] entwickelte Abbildung wird direkt ersichtlich, welcher SUSscore im akzeptablen, inakzeptablen oder marginalen Bereich liegt. Für jeden Stakeholder wurde jeweils für die Systembewertung der Vorversion und des Prototyps ein SUS Score berechnet. Die gesamte Systembewertung über alle Stakeholder, jeweils für Vorversion und Prototyp, wird 
durch drei Lagemaße bestimmt. Das zentrale Lagemaß bildet der Median, der die Stichprobe in zwei Hälften unterteilt. Die Werte der einen Hälfte sind größer, wohingegen die Werte der anderen kleiner als der Median sind [150].

Die beiden weiteren Lagemaße sind das 25\%- und 75\%-Quartil. Bei dem 25\%-Quartil sind 25\% der Werte unterhalb des Quartils und entsprechend 75\% beim 75\%-Quartil. Die Berechnung der Quartile erfolgt entsprechend dem Median, der auch als 50\%-Quartil bezeichnet wird [150]. Das 25\%- und 75\%-Quartil wurde nur für die Systembewertung des Prototyps verwendet, da die beiden Quartile bei der Bewertung der Vorversion systembedingt zu nahe am Median lagen. Dies begründete sich darin, dass nur fünf Stakeholder die Vorversion nutzen.

Zur Darstellung der Ergebnisse des System Usability Scale wurde die nachfolgende Tabelle 6 verwendet. Diese umfasst für alle fünf bzw. zehn Stakeholder die einzelnen Werte der zehn Fragen (F1 bis F10), den berechneten SUS score sowie die Lagemaße. Alle Stakeholder erhielten eine Nummer sowie ein Kürzel, welches beschreibt, ob es sich bei dem Stakeholder um einen Arzt (A), einen wissenschaftlichen Mitarbeiter (M) oder einen Studienassistenten (S) handelte. Die Angaben in Tabelle 6 entstammen dem Beispiel aus Anhang A.4.

\begin{tabular}{|c|c|c|c|c|c|c|c|c|c|c|c|}
\hline & $\mathbf{F}_{1}$ & $\mathbf{F}_{2}$ & $\mathbf{F}_{3}$ & $\mathbf{F}_{4}$ & $\mathbf{F}_{5}$ & $\mathbf{F}_{6}$ & $\mathbf{F}_{7}$ & $\mathbf{F}_{8}$ & F9 $_{9}$ & $\mathbf{F}_{10}$ & SUS Score \\
\hline Stakeholder & 4 & 1 & 1 & 4 & 1 & 2 & 1 & 1 & 4 & 3 & 55 \\
\hline & & & & & & & & \multicolumn{3}{|c|}{ Median } & 55 \\
\hline & & & & & & & & \multicolumn{3}{|c|}{ 25\%-Quartil } & 55 \\
\hline & & & & & & & & \multicolumn{3}{|c|}{ 75\%-Quartil } & 55 \\
\hline
\end{tabular}

Tabelle 6. Vorlage zur Darstellung der Ergebnisse des System Usability Scale 


\section{Anforderungsanalyse}

Im ersten Schritt der Anforderungsanalyse wurden Ziele an ein Probandenmanagementsystem für die Universitätsmedizin Göttingen identifiziert (siehe Abschnitt 3.1). Aus den gewonnen Zielen wurden Szenarien hergeleitet (siehe Abschnitt 3.2). Die identifizierten Szenarien bilden die Antwort auf die erste Forschungsfragestellung: „Welche Szenarien existieren für den Einsatz des zu konzeptionierenden Probandenmanagementsystems an der Universitätsmedizin Göttingen?“ Im letzten Schritt der Anforderungsanalyse wurden aus den identifizierten Zielen und Szenarien lösungsorientierte Anforderungen an das Probandenmanagementsystem gewonnen (siehe Abschnitt 3.3). Die gewonnenen Anforderungen bilden im nächsten Kapitel die Grundlage zur Konzeption des Systems (siehe Kapitel 4).

\subsection{Ziele}

Entsprechend der in Abschnitt 2.1.2 beschriebenen Methodik zur Auswertung der Interviews (siehe Anhang A.5) wurden 16 Ziele identifiziert. Diese sind zusammenfassend in Tabelle 7 dargestellt.

\begin{tabular}{l|l} 
Nr. & Ziel \\
\hline Z-1 & Überblick über alle Forschungsprojekte \\
\hline Z-2 & Dokumentation der genutzten Forschungssysteme \\
\hline Z-3 & Überblick über alle Probanden \\
\hline Z-4 & Einheitliche Erfassung von Probandenstammdaten \\
\hline Z-5 & Projektübergreifende Verwaltung von Probandenstammdaten \\
\hline Z-6 & Abruf von Probandenstammdaten aus SAP IS-H \\
\hline Z-7 & Übernahme von Probanden aus vorhandenen Forschungsprojekten \\
\hline Z-8 & Änderung von Probandenstammdaten \\
\hline Z-9 & Löschung von Probandenstammdaten \\
\hline Z-10 & Import von Probandenstammdaten \\
\hline Z-11 & Export von Probandenstammdaten \\
\hline Z-12 & Abbildung der Einwilligungserklärung \\
\hline Z-13 & Generierung von Pseudonymen \\
\hline Z-14 & Speicherung von Pseudonymen \\
\hline Z-15 & Abruf von Pseudonymen aus externen Systemen zur Pseudonymisierung \\
\hline Z-16 & Generierung von projektübergreifenden Forschungspseudonymen
\end{tabular}

Tabelle 7. Zusammenfassung der 16 identifizierten Ziele

Die identifizierten Ziele können thematisch in die Bereiche Verwaltung von Forschungsprojekten, Verwaltung von Probandenstammdaten sowie Pseudonymisierung eingeteilt werden.

\subsubsection{Verwaltung von Forschungsprojekten}

Die langfristige Verwaltung von Probanden erfordert von einem zu konzeptionierenden Probandenmanagementsystem einen Überblick über alle Forschungsprojekte (Z-1) an einer For- 
schungseinrichtung. Einher geht damit auch die Dokumentation der genutzten Forschungssysteme in den jeweiligen Forschungsprojekten (Z-2). Die Dokumentation soll festhalten, in welchen Forschungssystemen welche Forschungsdaten der Probanden gespeichert sind. Hierdurch soll die Transparenz und Vergleichbarkeit der Forschungsprojekte erhöht werden.

\subsubsection{Verwaltung von Probandenstammdaten}

Der Begriff Probandenstammdaten beschreibt die Zusammenstellung der persönlichen Angaben eines Probanden, wie beispielsweise Name, Geburtsdatum, Geschlecht oder Kontaktinformationen. Ein wichtiges Ziel für die Stakeholder ist eine Übersicht über all ihre Probanden (Z3) zu erhalten. Ebenso sollen eine einheitliche Erfassung (Z-4) sowie eine projektübergreifende Verwaltung (Z-5) der Probandenstammdaten sichergestellt sein. Hierdurch soll eine einheitliche Pflege der Probandenstammdaten über alle Forschungsprojekte im Probandenmanagementsystem ermöglicht werden.

In den Interviews (siehe Anhang A.5) wurde deutlich, dass ein Teil der Probanden direkt auf der Station rekrutiert wird. Dies bedeutet, dass die Probanden Patienten der Universitätsmedizin Göttingen sind und somit deren Stammdaten in SAP IS-H, dem klinisch-administrativen System des Krankenhausinformationssystems, hinterlegt sind. Der Abruf von Probandenstammdaten aus SAP IS-H (Z-6) ermöglicht die Wiederverwendung der vorhandenen Patientenstammdaten und vermeidet so eine erneute Eingabe dieser Daten in das Probandenmanagementsystem. Einen ähnlichen Ansatz verfolgt auch die Übernahme von Probanden aus vorhandenen Projekten (Z-7). Probandenstammdaten, die bereits in einem Forschungsprojekt erfasst wurden, sollen nicht nochmals erfasst werden.

Ergänzt werden diese Ziele durch die zentrale Änderung (Z-8) und Löschung (Z-9) von Probandenstammdaten. Die Interviews zeigten zudem, dass die Verwaltung der Probanden derzeit meist mit Excel-Tabellen erfolgt. Ein wichtiges Ziel ist daher der Import von Probandenstammdaten (Z-10) aus vorhandenen Datenquellen. Ebenso wichtig ist der Export von Probandenstammdaten (Z-11), sodass diese für Serienbriefe oder Statistiken genutzt werden können. Die Verwaltung von Probandenstammdaten ist an der Universitätsmedizin Göttingen, gemäß Niedersächsischem Datenschutzgesetz (NDSG), nur durch die Einwilligung des jeweiligen Probanden möglich (vgl. §4 Abs. 2 Satz 1 und §25 Abs. 2 NDSG). Ein Ziel bildet daher die Abbildung der Einwilligungserklärung (Z-12) des Probanden im Probandenmanagementsystem. Dieses Ziel ist Gegenstand der Masterarbeit von Markus Matoni, die im Forschungsumfeld dieser Dissertation erfolgte [118]. Auf die Erkenntnisse der Masterarbeit wird an dieser Stelle nur verwiesen. Eine Einarbeitung der Ergebnisse erfolgt nicht, da die Thematik der Einwilligungserklärungen ein umfangreiches Forschungsfeld darstellt.

\subsubsection{Pseudonymisierung}

In medizinischen Forschungsprojekten an der Universitätsmedizin Göttingen sollen die Probandenstammdaten möglichst gesondert von den Forschungsdaten gespeichert werden (vgl. 
$\S 25$ Abs. 4 NDSG). Die Zuordnung von Probanden zu deren Forschungsdaten erfolgt unter Verwendung von Pseudonymen [47, 152].

Aktuell wird in deutschen Forschungsprojekten die Verwendung mehrerer Pseudonyme für einen Probanden empfohlen [47]. Die Pseudonymisierung ist somit ein wichtiger Bestandteil des Probandenmanagementsystems. Ziele sind daher die Generierung von Pseudonymen (Z-13) sowie deren Speicherung (Z-14). In medizinischen Verbundforschungsprojekten erfolgt die Pseudonymisierung in der Regel durch ein zentrales System für alle Verbundpartner [41, 47]. Der Abruf von Pseudonymen aus solchen externen Systemen (Z-15) ist somit ein wichtiges Ziel, welches durch das Probandenmanagementsystem erfüllt werden muss. Gegenstand des letzten Ziels ist die (projektübergreifende) Zuordnung der verschiedenen Pseudonyme eines Probanden durch die Bereitstellung eines Forschungspseudonyms (Z-16). Die Generierung von projektübergreifenden Forschungspseudonymen wurde in der Bachelorarbeit von Martin Schwarzmaier erarbeitet [117].

\subsection{Szenarien}

Aus den 16 identifizierten Zielen wurden durch Anwendung der in Abschnitt 2.1.3 beschriebenen Methodik neun Szenarien hergeleitet (siehe Tabelle 8). Die beiden zentralen Akteure, welche in den Szenarien interagieren, sind die Mitarbeiter in einem Forschungsprojekt (Benutzer) und der IT-Betreuer des Probandenmanagementsystems (Administrator).

\begin{tabular}{l|l} 
Nr. & Szenario \\
\hline S-1 & Erfassung von Forschungsprojekten \\
\hline S-2 & Erfassung von Probanden \\
\hline S-3 & Änderung von Probandenstammdaten \\
\hline S-4 & Löschung eines Probanden \\
\hline S-5 & Import von Probandenstammdaten \\
\hline S-6 & Export von Probandenstammdaten \\
\hline S-7 & Pseudonymisierung \\
\hline S-8 & Abbildung der Einwilligungserklärung \\
\hline S-9 & Generierung von projektübergreifenden Forschungspseudonymen
\end{tabular}

Tabelle 8. Zusammenfassung der neun identifizierten Szenarien

\subsubsection{Erfassung von Forschungsprojekten}

Die Erfassung von Forschungsprojekten (S-1) leitet sich aus den zwei Zielen Überblick über alle Forschungsprojekte (Z-1) und Dokumentation auf die genutzten Forschungssysteme (Z-2) ab. Im Mittelpunkt des Szenarios steht die Konfiguration des Probandenmanagementsystems für das jeweilige Forschungsprojekt (siehe Abbildung 7). Hierfür wird durch den Administrator ein neues Forschungsprojekt im System angelegt. Dazu erfasst der Administrator alle relevanten Informationen über das Forschungsprojekt, bspw. Name, Typ, eingesetzte Forschungssysteme sowie die zukünftigen Benutzer und deren Rollen. Die Projektkonfiguration wird nach- 
folgend durch die zukünftigen Benutzer validiert und abgenommen. War die Abnahme erfolgreich, wird das Projekt durch den Administrator im Probandenmanagementsystem für die Benutzer freigeschaltet. Eine Änderung der Konfiguration des Forschungsprojekts ist ebenfalls möglich und würde ebenfalls mit der Abstimmung der Systemkonfiguration beginnen.

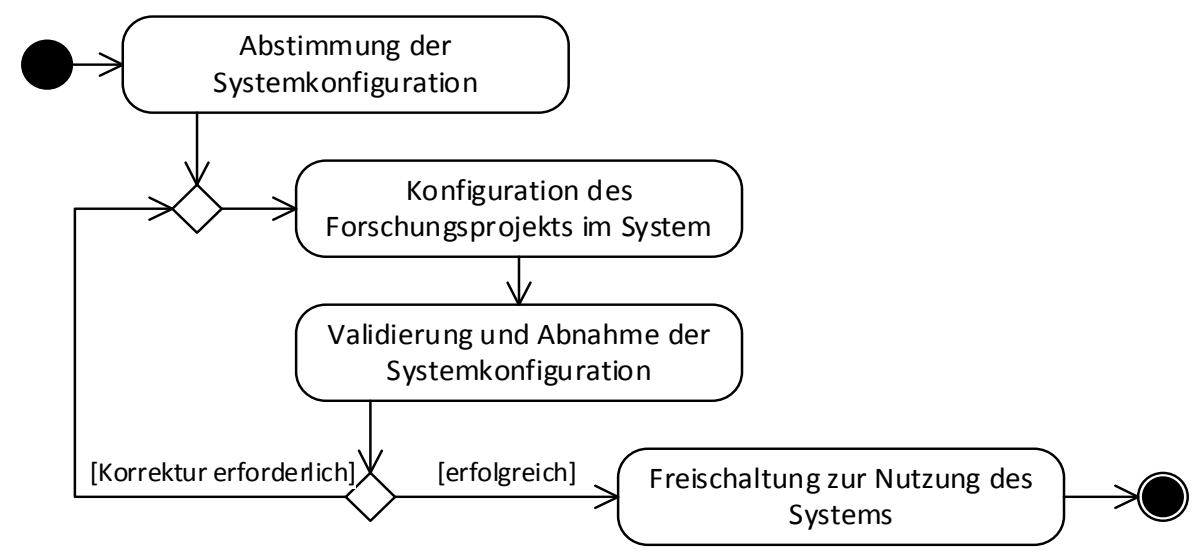

Abbildung 7. Erfassung von Forschungsprojekten (S-1)

\subsubsection{Erfassung von Probanden}

Das Szenario Erfassung von Probanden (S-2) bildet die Grundlage für eine effiziente und einheitliche Verwaltung sowie die langfristige Nachverfolgung von Probanden.

Dieses Szenario vereint die Ziele Überblick über alle Probanden (Z-3), einheitliche Erfassung von Probandenstammdaten (Z-4), projektübergreifende Verwaltung von Probandenstammdaten (Z-5), Abruf von Probandenstammdaten aus SAP IS-H (Z-6) sowie Übernahme von Probanden aus vorhandenen Forschungsprojekten (Z-7).

Zur Erfüllung der fünf Ziele sieht das Szenario vor, dass die Probandenstammdaten zentral durch das Probandenmanagementsystem verwaltet und den jeweiligen Forschungsprojekten lediglich zugeordnet werden. Den Benutzern werden drei Varianten zur Erfassung eines Probanden bereitgestellt (siehe Abbildung 8).

Die erste Variante sieht vor, dass dem Benutzer eine Übersicht von Probanden bereitgestellt wird, aus welcher er den betreffenden Probanden auswählen kann. Grundlage der Übersicht bilden die Probanden aller dem Benutzer zugeordneten Forschungsprojekte. Hierdurch wird eine Rekrutierung von Probanden aus bereits durchgeführten Forschungsprojekten vereinfacht.

Zur Unterstützung von versorgungsnahen Forschungsprojekten umfasst die zweite Variante die Erfassung von Probanden mittels der Patientenkennung des Krankenhauses. Die Verwaltung der Patientenkennung und der zugehörigen Patientenstammdaten erfolgt durch das klinischadministrative System des Krankenhauses [71]. Nach Eingabe der Patientenkennung wird zunächst geprüft, ob diese bereits im Probandenmanagementsystem vorhanden ist. Falls ja, wird der zugehörige Proband dem Forschungsprojekt zugeordnet. Sollte die Patientenkennung nicht 
bekannt sein, werden die Stammdaten anhand der Patientenkennung aus SAP IS-H abgerufen und der Proband neu angelegt.

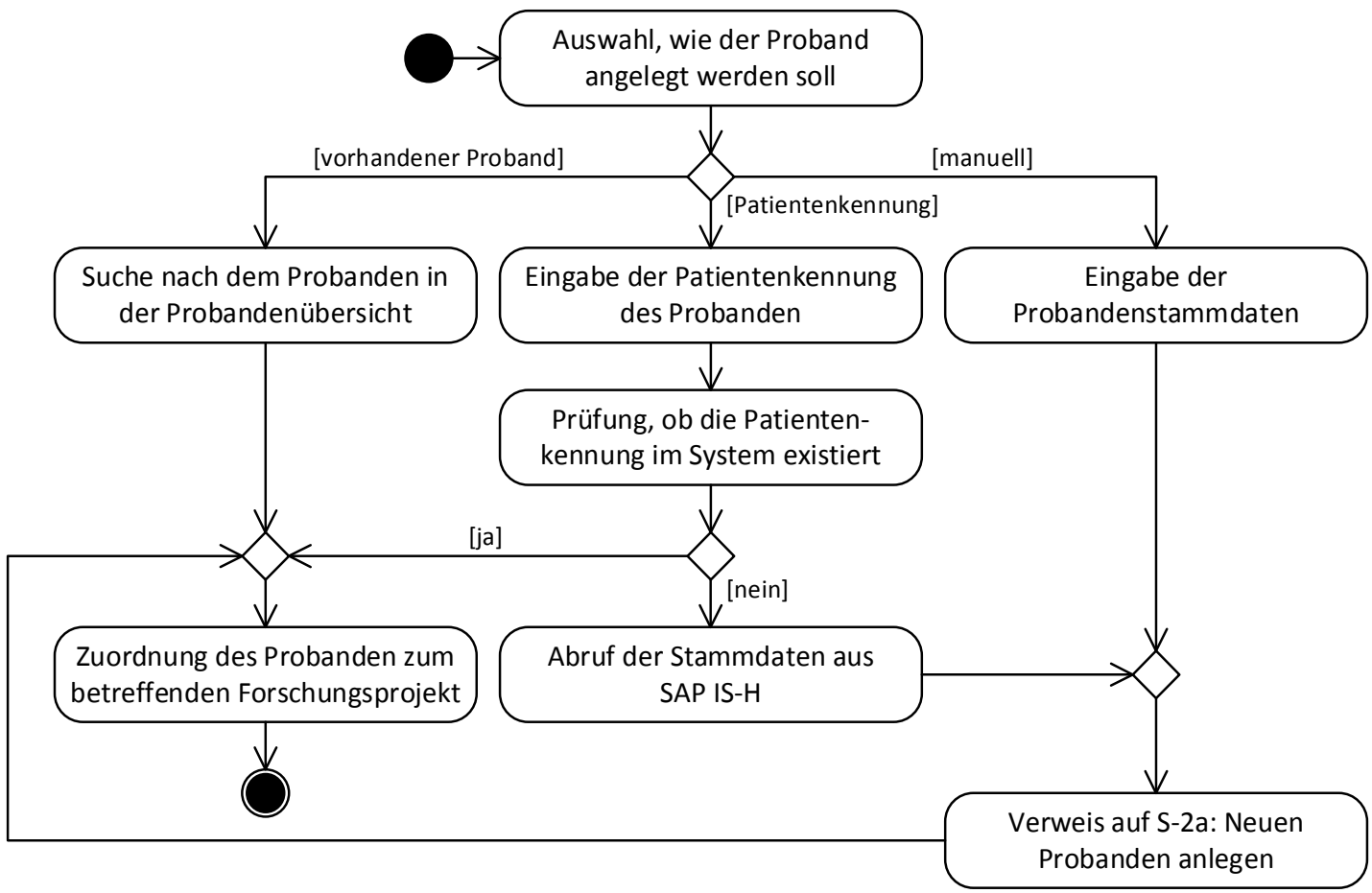

Abbildung 8. Erfassung von Probanden (S-2)

Die dritte Variante bietet dem Benutzer die Möglichkeit, einen Probanden anhand seiner Stammdaten anzulegen. Nach Eingabe der Probandenstammdaten prüft das System, ob der Proband bereits erfasst wurde. Aus diesem Teilbereich des Szenarios ergibt sich das Unterszenario Anlegen eines neuen Probanden (S-2a) in Abbildung 9.

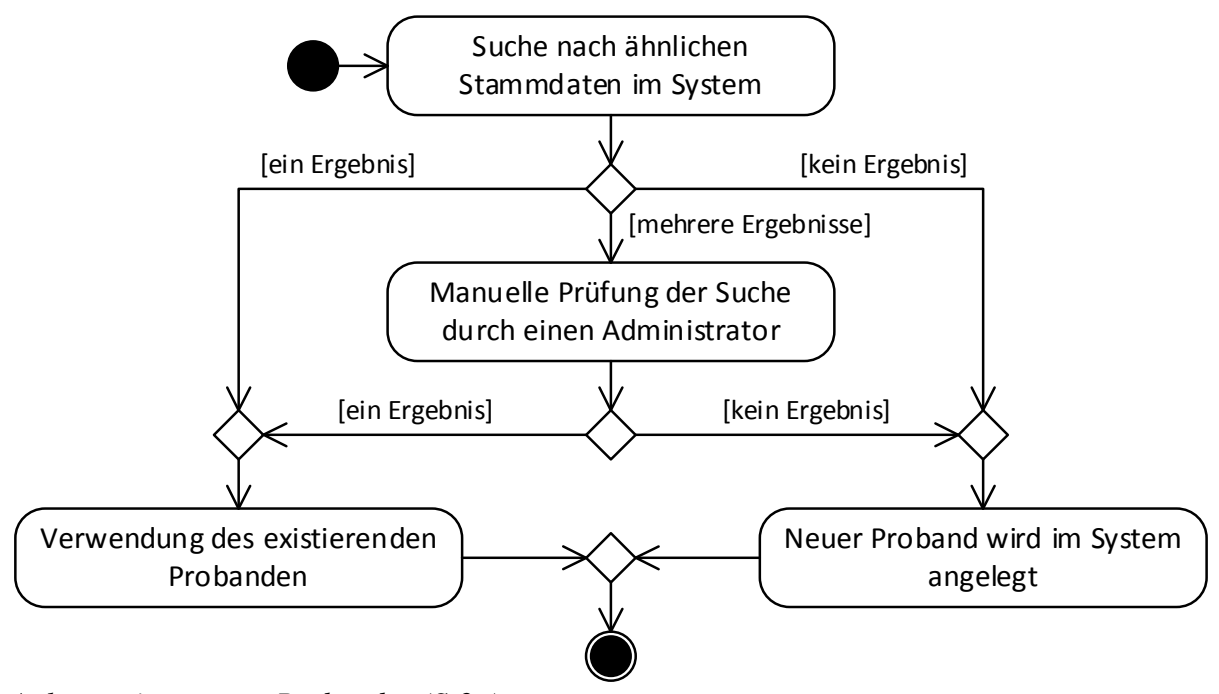

Abbildung 9. Anlegen eines neuen Probanden (S-2a)

Innerhalb des Unterszenarios wird geprüft, ob ein Proband mit gleichen oder ähnlichen Stammdaten im Probandenmanagementsystem bereits existiert, bevor dieser neu angelegt wird. Zunächst wird eine Suche nach ähnlichen Probandenstammdaten durchgeführt. Ergab die Suche 
kein Ergebnis, wird der Proband neu angelegt. Wurden hingegen mehrere ähnliche Probanden gefunden, werden die Suchergebnisse durch den Administrator manuell geprüft. Existiert ein identischer Proband im System, so wird dieser dem Forschungsprojekt zugeordnet und kein neuer Proband im System angelegt. Sollte kein identischer Proband im System existieren, so wird der Proband mit seinen Stammdaten neu im Probandenmanagementsystem angelegt.

\subsection{3 Änderung von Probandenstammdaten}

Die Sicherstellung einer langfristigen Nachverfolgung von Probanden erfordert die Änderung von Probandenstammdaten (S-3) bspw. aufgrund eines Namenswechsels oder Umzugs. Dieses Szenario erfüllt das gleichnamige Ziel Änderung von Probandenstammdaten (Z-8). Zur Sicherstellung der projektübergreifenden Verwaltung von Probandenstammdaten ist eine direkte Änderung der Stammdaten nur durch einen Administrator möglich (siehe Abbildung 10).

Ein Benutzer wendet sich zur Änderung der Stammdaten eines Probanden an den Administrator des Probandenmanagementsystems. Vor der Änderung wird eine Suche nach identischen Probanden, die bereits im System erfasst wurden, durchgeführt. Sollte das Suchergebnis nicht eindeutig sein, erfolgt eine manuelle Prüfung durch den Administrator. Die Änderungen an den Stammdaten werden nur übernommen, sofern kein identischer Proband gefunden wurde. Sollte ein identischer Proband existieren, werden beide Probanden zusammengeführt. Eine Änderung von Probandenstammdaten aus dem klinisch-administrativen System kann nicht im Probandenmanagementsystem durchgeführt werden, sondern muss im klinisch-administrativen System vorgenommen werden.

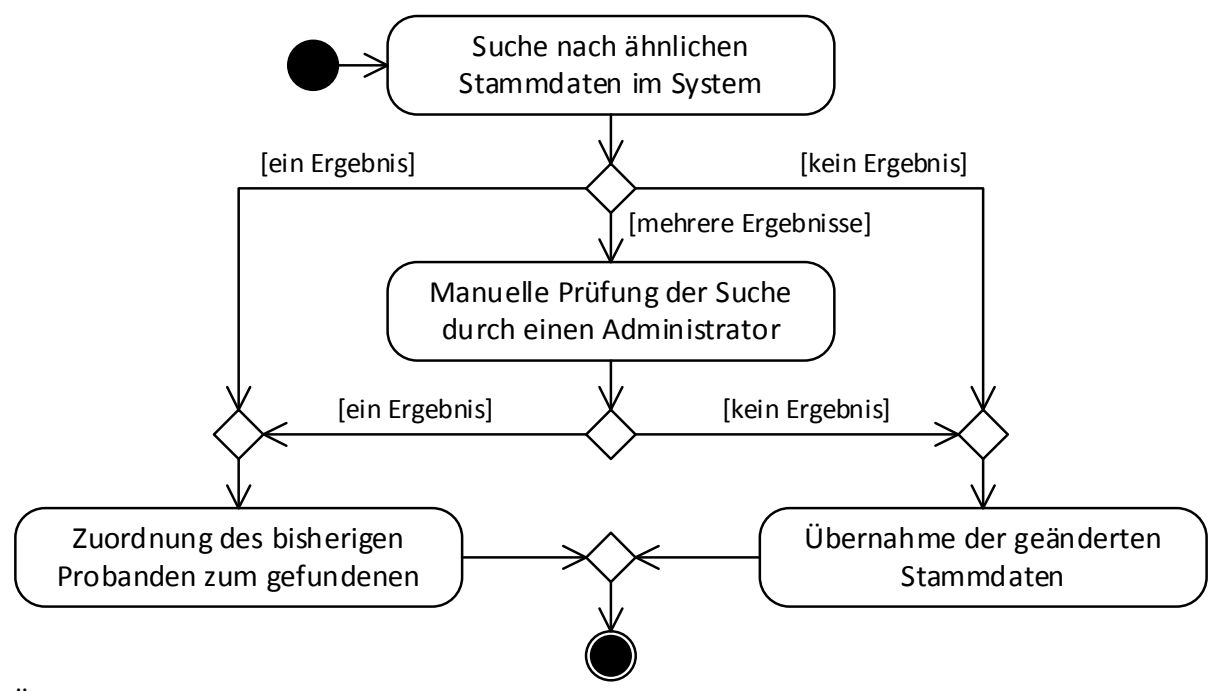

Abbildung 10. Änderung von Probandenstammdaten (S-3)

\subsubsection{Löschung eines Probanden}

Das Ziel Löschung von Probanden (Z-9) wird durch das Szenario Löschung eines Probanden (S-4) erfüllt. Dieses Szenario tritt beispielsweise ein, wenn die Einwilligungserklärung zur Teilnahme am Forschungsprojekt zurückgezogen wird oder der Proband fälschlicherweise angelegt 
wurde. Ist die Löschung eines Probanden erforderlich, wendet sich der Benutzer an den Administrator. Dieser führt die Löschung des Probanden aus (siehe Abbildung 11). Zunächst wird die Zuordnung des Probanden zum Forschungsprojekt gelöscht. Sollte der Proband danach keinem Forschungsprojekt mehr zugeordnet sein, werden seine Daten vollständig aus dem Probandenmanagementsystem entfernt.

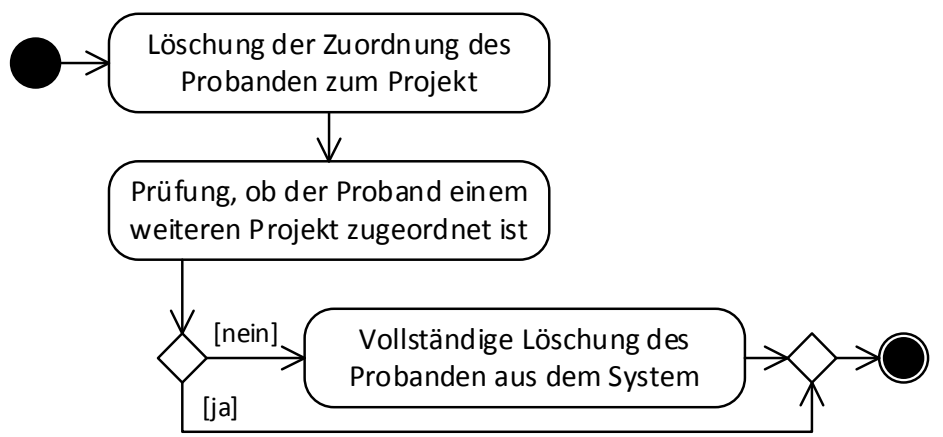

Abbildung 11. Löschung eines Probanden (S-4)

\subsubsection{Import von Probandenstammdaten}

Das Szenario Import von Probandenstammdaten (S-5) erfüllt das gleichnamige Ziel (Z-10). Es bietet Forschungsprojekten eine effiziente Möglichkeit, bereits erfasste Probanden in das Probandenmanagementsystem zu importieren (siehe Abbildung 12).

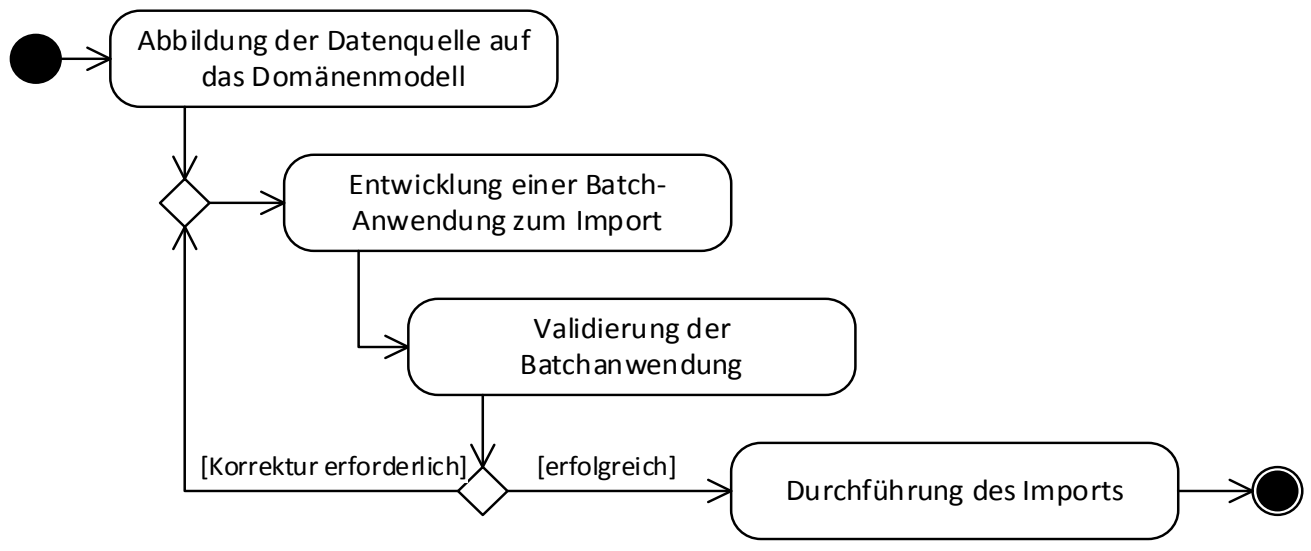

Abbildung 12. Import von Probandenstammdaten (S-5)

Dies erfolgt durch den Administrator des Probandenmanagementsystems. Er bildet die zu importierende Datenquelle auf das Datenmodell des Probandenmanagementsystems ab. Auf Grundlage dieser Abbildung erfolgt die Entwicklung einer Batch-Anwendung zum Import der Daten. Nach erfolgreicher Validierung der Batch-Anwendung wird der Datenimport durchgeführt.

\subsubsection{Export von Probandenstammdaten}

Dem Szenario Export von Probandenstammdaten (S-6) und dem gleichnamigen Ziel (Z-11) liegt zugrunde, dass es im Verlauf eines Forschungsprojekts notwendig ist, Probandenstammdaten beispielsweise für einen Serienbrief zu exportieren. Die benötigten Datensätze werden 
durch den Administrator auf das Domänenmodell des Probandenmanagementsystems abgebildet. Nachfolgend wird ein entsprechender Export erstellt (siehe Abbildung 13).

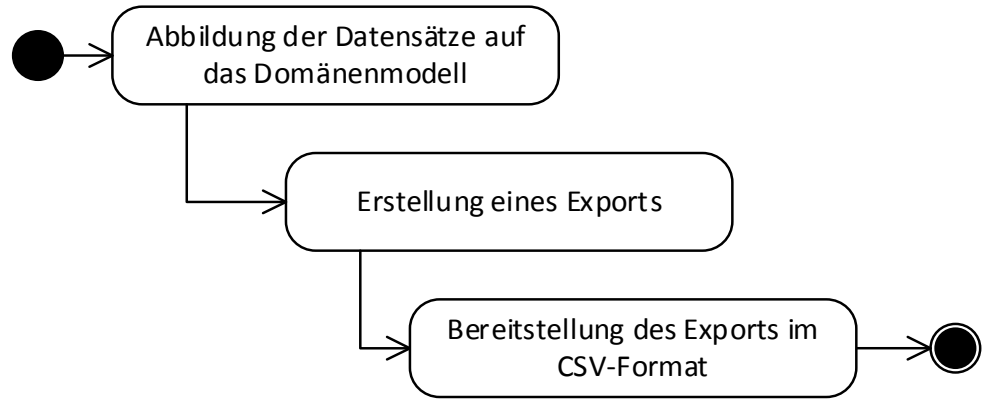

Abbildung 13. Export von Probandenstammdaten (S-6)

\subsubsection{Abbildung der Einwilligungserklärung}

Entsprechend der vorangegangen Erläuterung zum Ziel Abbildung der Einwilligungserklärung (Z-12), erfordert die Teilnahme eines Probanden an einem medizinischen Forschungsprojekt der Universitätsmedizin Göttingen dessen informierte Einwilligung (vgl. §25 Abs. 2 NDSG). Diese wird nach einer ausführlichen Erläuterung durch einen Mitarbeiter des Forschungsprojekts vom Probanden eingeholt. Gemäß §4 Abs. 2 Satz 1 NDSG bedarf dies an der Universitätsmedizin Göttingen der Schriftform. Inhalt des Szenarios Abbildung der Einwilligungserklärung (S-7) und zur Erfüllung des gleichnamigen Ziels (Z-12) ist die Übertragung der papierbasierten Einwilligung in das Probandenmanagementsystem, ohne die papierbasierte Einwilligungserklärung zu ersetzen (siehe Abbildung 14).

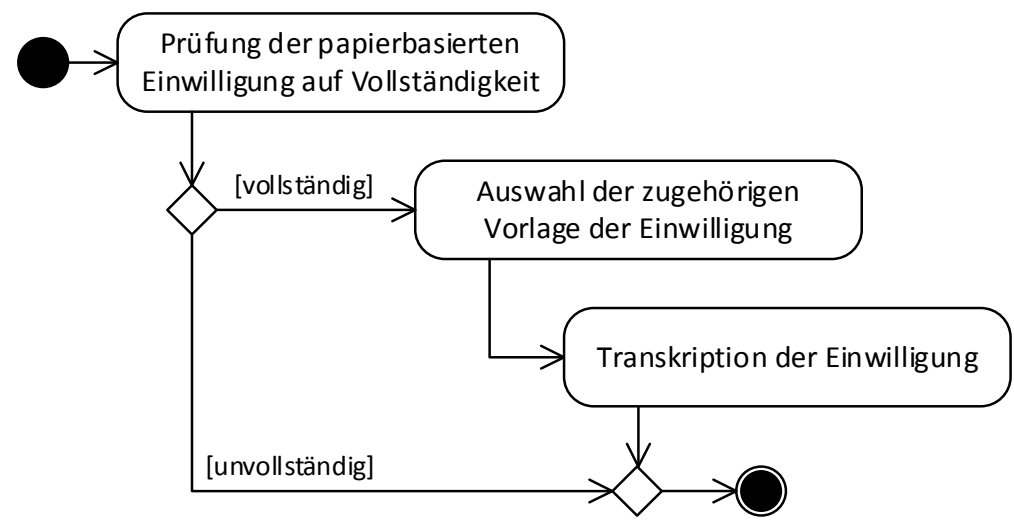

Abbildung 14. Abbildung der Einwilligungserklärung (S-7)

Eine ausführliche Beschreibung dieses Szenarios und dessen prototypische Implementierung in einem Probandenmanagementsystem ist Gegenstand der Masterarbeit von Markus Matoni, die im Forschungsumfeld dieser Dissertation erarbeitet wurde [118]. Daher werden nachfolgend nur die wesentlichen Aspekte des Szenarios beschrieben. 
Die Abbildung der Einwilligungserklärung im Probandenmanagementsystem erfordert zunächst die Erstellung einer Vorlage für die spätere Transkription. Nach Prüfung der papierbasierten Einwilligung durch den Benutzer überträgt dieser die Angaben in der papierbasierten Einwilligung gemäß der erstellten Vorlage.

\subsubsection{Pseudonymisierung}

Das Szenario Pseudonymisierung (S-8) erfüllt die Ziele Generierung von Pseudonymen (Z-13), Speicherung von Pseudonymen (Z-14) und Abruf von Pseudonymen aus externen Systemen (Z-15). In medizinischen Forschungsprojekten an der Universitätsmedizin Göttingen sollen die personenbezogenen Daten von Probanden (Probandenstammdaten) gesondert von den Forschungsdaten gespeichert werden (vgl. §25 Abs. 4 NDSG). Diese Vorgabe wird durch den Einsatz von Pseudonymen umgesetzt (siehe Abbildung 15).

Ein interner Pseudonymgenerator ermöglicht die Generierung von Pseudonymen durch das Probandenmanagementsystem. Ebenso ist auch eine manuelle Eingabe eines Pseudonyms durch einen Benutzer möglich. Die letzte Variante umfasst den Abruf eines Pseudonyms von einem externen System zur Pseudonymisierung. Nachdem ein Pseudonym für den Probanden erstellt bzw. abgerufen wurde, wählt der Benutzer abschließend aus, in welchen Forschungssystemen das Pseudonym verwendet wird.

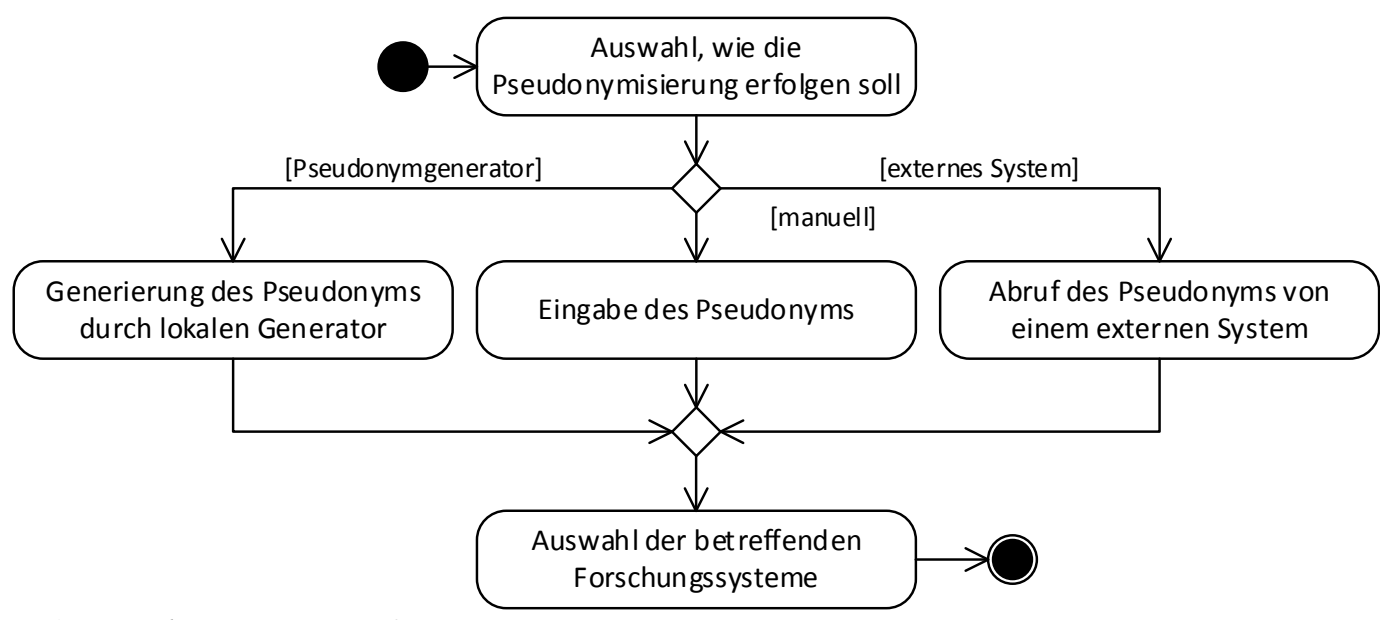

Abbildung 15. Pseudonymisierung (S-8)

\subsubsection{Generierung von projektübergreifenden Forschungspseudonymen}

In medizinischen Forschungsprojekten werden unterschiedliche Forschungsdaten über einen Probanden erfasst. Diese Daten werden unter Verwendung von Pseudonymen in spezifischen und optimierten Forschungssystemen gespeichert [115, 116].

Die Intention des zu erfüllenden Ziels Generierung von projektübergreifenden Forschungspseudonymen ist die Zusammenführung von Forschungsdaten aus verschiedenen Forschungssystemen und über mehrere Forschungsprojekte hinweg zu ermöglichen. Dies wird in Szenario (S9) abgebildet. Das Probandenmanagementsystem besitzt die notwendigen Informationen über 
verschiedene Pseudonyme eines Probanden. Dieses Szenario und dessen prototypische Implementierung ist Gegenstand der Bachelorarbeit von Martin Schwarzmaier [117]. Die Bachelorarbeit wurde im Forschungsumfeld dieser Dissertation erstellt, daher wird nachfolgend nur auf die wichtigsten Aspekte des Szenarios eingegangen (siehe Abbildung 16).

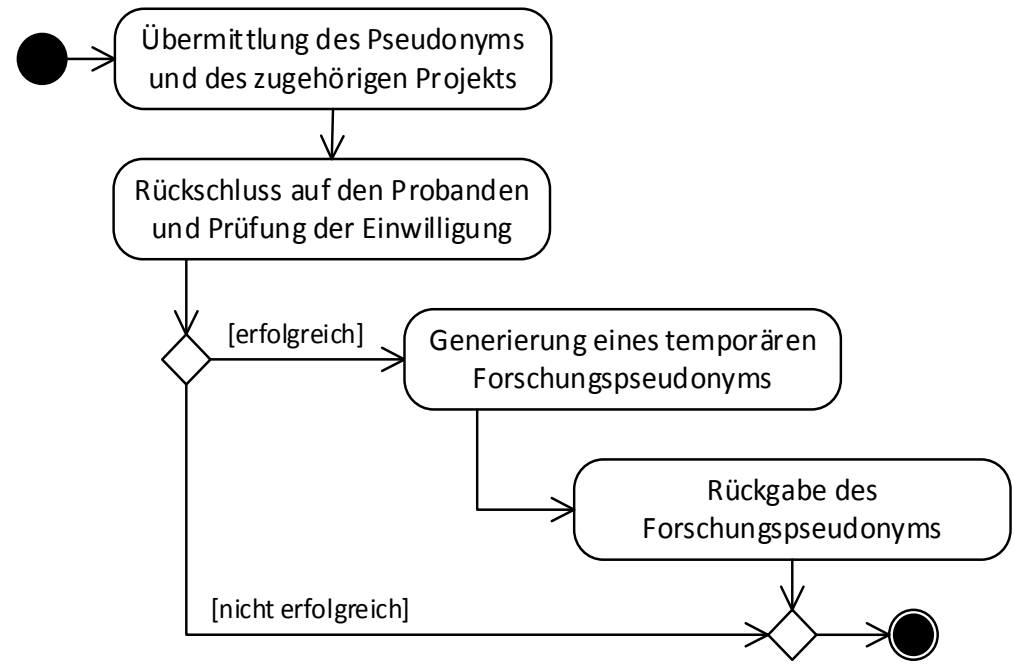

Abbildung 16. Generierung von projektübergreifenden Forschungspseudonymen (S-9)

Die Zusammenführung der Forschungsdaten erfolgt durch ein separates Softwarewerkzeug und nicht durch das Probandenmanagementsystem. Das Probandenmanagementsystem nimmt lediglich Pseudonyme durch eine definierte Schnittstelle entgegen und gibt ein zugehöriges Forschungspseudonym zurück. Nach Entgegennahme des Pseudonyms wird zunächst geprüft, ob die Einwilligung des Probanden für diese Datenzusammenführung vorliegt. Ist dies der Fall, prüft das System, ob bereits ein Forschungspseudonym existiert, andernfalls wird ein temporäres Forschungspseudonym generiert. Dies wird an das anfragende Softwarewerkzeug für die Datenzusammenführung zurückgegeben. Alle Pseudonyme eines Probanden erhalten das gleiche temporäre Forschungspseudonym.

\subsection{Lösungsorientierte Anforderungen}

Insgesamt wurden 46 lösungsorientierte Anforderungen aus den Anforderungsquellen gewonnen: 30 funktionale Anforderungen (Abschnitt 3.3.1), sieben Qualitätsanforderungen (Abschnitt 3.3.2) und neun Randbedingungen (Abschnitt 3.3.3).

\subsubsection{Funktionale Anforderungen}

Die gewonnenen 30 funktionalen Anforderungen wurden zur besseren Übersicht in fünf Kategorien unterteilt: Authentifizierung und Autorisierung, Verwaltung von Forschungsprojekten, Verwaltung von Probanden, Verwaltung von Einwilligungserklärungen und Pseudonymisierung. 


\section{Authentifizierung und Autorisierung}

Die Kategorie umfasst drei funktionale Anforderungen im Bereich der Authentifizierung und Autorisierung von Benutzern im Probandenmanagementsystem (siehe Tabelle 9).

\begin{tabular}{l|l|l} 
Nr. & Beschreibung & Quelle \\
\hline A-F-1 & $\begin{array}{l}\text { Die Authentifizierung der Benutzer soll über die zentrale Be- } \\
\text { nutzerverwaltung der Universitätsmedizin Göttingen erfolgen. }\end{array}$ & I-3, I-6, I-9 \\
\hline A-F-2 & $\begin{array}{l}\text { Alternativ soll für Benutzer, die nicht über die zentrale Benut- } \\
\text { zerverwaltung der Universitätsmedizin Göttingen verwaltet } \\
\text { werden, eine systeminterne Authentifizierung erfolgen. }\end{array}$ & I-11, I-12, [1] \\
\hline A-F-3 & $\begin{array}{l}\text { Ein feingranulares Rechte- und Rollenmanagement zur Auto- } \\
\text { risierung soll den Zugriff auf das System und dessen Funktio- } \\
\text { nen für Benutzer leicht konfigurierbar machen. }\end{array}$ & I-3, [1, 90, 153]
\end{tabular}

Tabelle 9. Lösungsorientierte funktionale Anforderungen der Kategorie Authentifizierung und Autorisierung

Wenn möglich, soll die Authentifizierung des Benutzers über die zentrale Benutzerverwaltung der Universitätsmedizin Göttingen durchgeführt werden (A-F-1). Ist dies nicht möglich, soll die Authentifizierung direkt im Probandenmanagementsystem erfolgen (A-F-2). Vorteil der zentralen Benutzerverwaltung ist, dass der Benutzer seine vorhandenen Zugangsdaten der Universitätsmedizin Göttingen zur Anmeldung am System verwenden kann. Das Kennwort unterliegt den Richtlinien (Gültigkeit, Länge und Format) der Universitätsmedizin Göttingen.

Die Zugriffsbeschränkungen für Benutzer sollen durch ein feingranulares Rechte- und Rollenmanagement abgebildet werden (A-F-3). Hierdurch soll die Autorisierung der Benutzer im Probandenmanagementsystem leicht konfigurierbar sein.

\section{Verwaltung von Forschungsprojekten}

Die Kategorie Verwaltung von Forschungsprojekten umfasst fünf funktionale Anforderungen (siehe Tabelle 10).

Primäre Anforderung dieser Kategorie ist, dass das Probandenmanagementsystem mehrere Forschungsprojekte verwalten soll (A-F-4). Zusätzlich sollen Metadaten zu den Forschungsprojekten erfasst werden (A-F-5). Dies wären beispielsweise die genutzten Forschungssysteme im jeweiligen Projekt.

Das Rechte- und Rollenmanagement soll für jedes Projekt einzeln konfigurierbar sein (A-F-6). Dies soll die Abbildung der realen Mitarbeiterrollen des Forschungsprojekts im System ermöglichen. Ebenso soll das Probandenmanagementsystem einem Benutzer ermöglichen mehreren Forschungsprojekten zugeordnet zu sein (A-F-7). Eng damit verbunden ist auch die Anforderung nach einer Übersicht über alle Forschungsprojekte eines Benutzers (A-F-8). 


\begin{tabular}{l|l|l} 
Nr. & Beschreibung & Quelle \\
\hline A-F-4 & $\begin{array}{l}\text { Das System soll mehrere Forschungsprojekte verwalten. Dies } \\
\text { umfasst das Anlegen, Bearbeiten und Löschen von For- } \\
\text { schungsprojekten. }\end{array}$ & S-1, [1] \\
\hline A-F-5 & $\begin{array}{l}\text { Das System soll Metadaten über Forschungsprojekte erfassen: } \\
\text { Angaben über die genutzten Forschungssysteme sowie den } \\
\text { aktuellen Status der Forschungsprojekte. }\end{array}$ & Z-2, [94] \\
\hline A-F-6 & $\begin{array}{l}\text { Das System soll sicherstellen, dass die Rechte und Rollen für } \\
\text { jedes Forschungsprojekt einzeln konfigurierbar sind. }\end{array}$ & I-3, S-1, [1] \\
\hline A-F-7 & $\begin{array}{l}\text { Das System soll den Benutzern die Möglichkeit bieten, meh- } \\
\text { reren Forschungsprojekten zugeordnet zu sein. }\end{array}$ & S-1, [1] \\
\hline A-F-8 & $\begin{array}{l}\text { Das System soll den Benutzern eine Übersicht über all ihre } \\
\text { Forschungsprojekte ermöglichen. }\end{array}$ & S-1
\end{tabular}

Tabelle 10. Lösungsorientierte funktionale Anforderungen der Kategorie Verwaltung von Forschungsprojekten

\section{Verwaltung von Probanden}

Insgesamt umfasst die Kategorie Verwaltung von Probanden elf lösungsorientierte funktionale Anforderungen (siehe Tabelle 11).

Die zentrale Anforderung ist die projektübergreifende Verwaltung der Probandenstammdaten (A-F-9 und A-F-10). Probandenstammdaten umfassen Vorname, Nachname, Geburtsdatum, Geschlecht, Geburtsname sowie Kontaktinformationen des Probanden. Auf Grund der projektübergreifenden Verwaltung soll die Änderung und Löschung der Probandenstammdaten nur durch einen Administrator möglich sein (A-F-11). Dies ist notwendig, da Probanden einem oder mehreren Forschungsprojekten zugeordnet sein können (A-F-10). Die Zuordnung eines Probanden zu einem Forschungsprojekt repräsentiert die Teilnahme des Probanden am Forschungsprojekt. Folglich kann ein Proband nur vollständig gelöscht werden, wenn er keinem Forschungsprojekt mehr zugeordnet ist (A-F-12).

Teilweise werden Probanden direkt auf der Station rekrutiert. Die Probanden sind somit Patienten der Universitätsmedizin Göttingen, sodass ihre Stammdaten im klinisch-administrativen System SAP IS-H geführt werden. Zur Vermeidung von Mehrfacheingaben soll daher das Anlegen eines Probanden mit dessen Patientenkennung erfolgen (A-F-13). Die notwendigen Stammdaten werden in diesem Fall über SAP IS-H abgerufen. Eine weitere Anforderung zur Vermeidung von Mehrfacheingaben ist die Übernahme von bereits erfassten Probanden von einem Forschungsprojekt in ein anderes (A-F-14). Alternativ sollen Probanden auch mittels manueller Eingabe der Stammdaten durch einen Benutzer angelegt werden können (A-F-15). Beim Anlegen eines Probanden soll automatisch eine Duplikaterkennung vorgenommen werden (A-F-16). Die Duplikaterkennung soll sicherstellen, dass ein Proband nicht mehrfach im Probandenmanagementsystem erfasst wird. 


\begin{tabular}{|c|c|c|}
\hline Nr. & Beschreibung & Quelle \\
\hline A-F-9 & $\begin{array}{l}\text { Das System soll die Probandenstammdaten verwalten. Diese } \\
\text { umfassen Vorname, Nachname, Geburtsdatum, Geschlecht, } \\
\text { Geburtsname sowie Kontaktinformationen. }\end{array}$ & $\begin{array}{l}\text { S-2, S-3, S-4, [1, } \\
89,91,97,153]\end{array}$ \\
\hline A-F-10 & $\begin{array}{l}\text { Das System soll sicherstellten, dass Probanden an mehreren } \\
\text { Forschungsprojekten teilnehmen können. }\end{array}$ & S-2 \\
\hline A-F-11 & $\begin{array}{l}\text { Die Änderung und Löschung der Probandenstammdaten soll } \\
\text { nur durch einen Administrator möglich sein. }\end{array}$ & Z-5, Z-8, Z-9 \\
\hline A-F-12 & $\begin{array}{l}\text { Die vollständige Löschung eines Probanden soll nur möglich } \\
\text { sein, wenn der Proband keinem weiteren Forschungsprojekt } \\
\text { zugeordnet ist. }\end{array}$ & S-4 \\
\hline A-F-13 & $\begin{array}{l}\text { Das Anlegen eines Probanden soll mittels seiner Patientenken- } \\
\text { nung der Universitätsmedizin Göttingen möglich sein. An- } \\
\text { hand der Patientenkennung soll ein Abruf der Stammdaten aus } \\
\text { SAP IS-H erfolgen. }\end{array}$ & S-2 \\
\hline A-F-14 & $\begin{array}{l}\text { Das Anlegen eines Probanden soll durch Übernahme aus ei- } \\
\text { nem im System bestehenden Forschungsprojekt möglich sein. }\end{array}$ & S-2 \\
\hline A-F-15 & $\begin{array}{l}\text { Das Anlegen eines Probanden soll mittels Eingabe der Pro- } \\
\text { bandenstammdaten möglich sein. }\end{array}$ & S-2 \\
\hline A-F-16 & $\begin{array}{l}\text { Beim Anlegen eines Probanden soll durch eine automatische } \\
\text { Duplikaterkennung sichergestellt werden, dass ein Proband } \\
\text { nicht mehrfach im System angelegt wird. }\end{array}$ & S-2, [2, 47] \\
\hline A-F-17 & $\begin{array}{l}\text { Das System soll den Benutzern eine Übersicht über all ihre } \\
\text { den Forschungsprojekten zugeordneten Probanden ermögli- } \\
\text { chen. }\end{array}$ & Z-1, Z-3 \\
\hline A-F-18 & $\begin{array}{l}\text { Die Zuordnung des Probanden zu einem Forschungsprojekt } \\
\text { soll auch den Status des Probanden im Projekt umfassen. }\end{array}$ & $\begin{array}{l}\mathrm{I}-4, \mathrm{I}-12, \mathrm{I}-14, \mathrm{I}- \\
16,[1]\end{array}$ \\
\hline A-F-19 & $\begin{array}{l}\text { Das System soll geeignete Schnittstellen zum Export und Im- } \\
\text { port von Probandenstammdaten vorhalten. }\end{array}$ & S-5, S-6, [1] \\
\hline
\end{tabular}

Tabelle 11. Lösungsorientierte funktionale Anforderungen der Kategorie Verwaltung von Probanden

Generell soll dem Benutzer eine Übersicht über all seine Probanden bereitgestellt werden (AF-17). Die Übersicht ergibt sich aus der Zuordnung des Benutzers zu seinen Forschungsprojekten, welchen wiederum Probanden zugeordnet sind. In dieser Zuordnung soll auch der aktuelle Status des Probanden im Forschungsprojekt hinterlegt werden, bspw. dass er eingeschlossen wurde oder dass alle Untersuchungen abgeschlossen sind (A-F-18). Das Probandenmanagementsystem soll geeignete Schnittstellen zum Export und Import der Probandenstammdaten bereitstellen (A-F-19), um so auf die konkreten Bedürfnisse der Benutzer einzugehen. 


\section{Verwaltung von Einwilligungserklärungen}

Markus Matoni beschreibt in seiner Masterarbeit umfassend die Anforderungen zur Verwaltung von Einwilligungserklärungen in einem Probandenmanagementsystem. Die fünf Anforderungen dieser Kategorie werden nachfolgend kurz erläutert (siehe Tabelle 12). Eine ausführliche Beschreibung findet sich in der Masterarbeit [118].

Eine Abbildung der papierbasierten Einwilligung erfolgt auf Grundlage der Zuordnung des Probanden zum jeweiligen Forschungsprojekt (A-F-20). Ausgangspunkt dieser Abbildung bildet eine Vorlage, die für jedes Forschungsprojekt separat modelliert werden soll (A-F-21). Die Vorlage besteht aus verschiedenen Modulen (A-F-22). Ein Modul entspricht einem Abschnitt der papierbasierten Einwilligungserklärung. Nachdem die Abbildung der Einwilligung erfolgte, soll dem Benutzer die Möglichkeit gegeben werden, die papierbasierte Einwilligung als Anhang zur elektronischen Abbildung zu hinterlegen (A-F-23).

\begin{tabular}{l|l|l} 
Nr. & Beschreibung & Quelle \\
\hline A-F-20 & $\begin{array}{l}\text { Das System soll die Abbildung der Einwilligungserklärung er- } \\
\text { möglichen. }\end{array}$ & S-7, [1, 2, 118] \\
\hline A-F-21 & $\begin{array}{l}\text { Einwilligungserklärungen sollen dabei auf Vorlagen basieren, } \\
\text { die für jedes Forschungsprojekt einzeln modelliert werden sol- } \\
\text { len. }\end{array}$ & $\begin{array}{l}\text { S-7, [1, 118, } \\
154]\end{array}$ \\
\hline A-F-22 & $\begin{array}{l}\text { Die Vorlage einer Einwilligungserklärung soll aus verschiede- } \\
\text { nen Modulen bestehen. Ein Modul entspricht einem Abschnitt } \\
\text { in der Einwilligungserklärung. }\end{array}$ & $\begin{array}{l}\text { S-7, }[1,18, \\
154]\end{array}$ \\
\hline A-F-23 & $\begin{array}{l}\text { Die papierbasierte Einwilligungserklärung eines Probanden } \\
\text { soll als Anhang zu der elektronischen Abbildung der Einwilli- } \\
\text { gungserklärung im System hinterlegt werden. }\end{array}$ & {$[1,118,154]$} \\
\hline A-F-24 & $\begin{array}{l}\text { Das System soll den Widerruf einer Einwilligungserklärung } \\
\text { durch einen Probanden abbilden. }\end{array}$ & {$[1,118,154]$}
\end{tabular}

Tabelle 12. Lösungsorientierte funktionale Anforderungen der Kategorie Verwaltung von Einwilligungserklärungen

Darüber hinaus soll das Probandenmanagementsystem auch den Widerruf einer Einwilligungserklärung durch den Probanden abbilden (A-F-24). Die Probanden haben das Recht, die Einwilligung in ein Forschungsprojekt jederzeit und ohne Angabe von Gründen zu widerrufen (vgl. $\S 4$ Abs. 2 Satz 5 NDSG).

\section{Pseudonymisierung}

Sechs funktionale Anforderungen sind Gegenstand der letzten Kategorie Pseudonymisierung (siehe Tabelle 13).

Das Probandenmanagementsystem soll die Erfassung, Bearbeitung und Löschung von Pseudonymen für Probanden ermöglichen (A-F-25). Ausgangspunkt ist die Zuordnung des Probanden zum jeweiligen Forschungsprojekt. Nach der Erstellung eines Pseudonyms soll das zugehörige 
Forschungssystem erfasst werden, in welchem das Pseudonym verwendet wird (A-F-26). Typischerweise werden in einem Forschungsprojekt für einen Probanden unterschiedliche Pseudonyme für die jeweiligen Forschungssysteme verwendet [47]. Benutzer sollen daher die Möglichkeit erhalten, Probanden anhand ihrer Pseudonyme im System zu suchen (A-F-27)

\begin{tabular}{l|l|l} 
Nr. & Beschreibung & Quelle \\
\hline A-F-25 & $\begin{array}{l}\text { Das System soll die Erfassung, Bearbeitung und Löschung } \\
\text { von Pseudonymen für Probanden ermöglichen. }\end{array}$ & S-8, [1] \\
\hline A-F-26 & $\begin{array}{l}\text { Das System soll bei der Erstellung eines Pseudonyms die zu- } \\
\text { gehörigen Forschungssysteme erfassen. }\end{array}$ & I-14, S-8, [117] \\
\hline A-F-27 & $\begin{array}{l}\text { Das System soll den Benutzern die Möglichkeit bieten Pro- } \\
\text { banden anhand ihrer Pseudonyme zu suchen. }\end{array}$ & I-9, I-10, [117] \\
\hline A-F-28 & $\begin{array}{l}\text { Das System soll den Benutzern die Möglichkeit bieten Pseu- } \\
\text { donyme für einen Probanden zu generieren. }\end{array}$ & S-8 \\
\hline A-F-29 & $\begin{array}{l}\text { Das System soll den Benutzern die Möglichkeit bieten Pseu- } \\
\text { donyme von einem externen System abzurufen. }\end{array}$ & $\begin{array}{l}\text { S-8 , [1, 2, } \\
41,47]\end{array}$ \\
\hline A-F-30 & $\begin{array}{l}\text { Das System soll Benutzern die Möglichkeit bieten Pseudo- } \\
\text { nyme eines Probanden aus mehreren Forschungsprojekten } \\
\text { mittels eines projektübergreifenden Forschungspseudonyms } \\
\text { zuzuordnen. }\end{array}$ & $\begin{array}{l}\text { S-9, I-9, I-11, } \\
{[117]}\end{array}$ \\
\hline
\end{tabular}

Tabelle 13. Lösungsorientierte funktionale Anforderungen der Kategorie Pseudonymisierung

Generell soll das System die Benutzer nicht nur bei der Erfassung, sondern auch bei der Generierung von Pseudonymen unterstützen (A-F-28). Eine weitere sehr wichtige Anforderung ist der Abruf von Pseudonymen aus externen Systemen (A-F-29), die im Bereich der medizinischen Verbundforschung eingesetzt werden [2, 47].

Die letzte funktionale Anforderung (A-F-30) ist Gegenstand der Bachelorarbeit von Martin Schwarzmaier und umfasst die projektübergreifende Zuordnung von verschiedenen Pseudonymen eines Probanden unter einem neuen Forschungspseudonym [117]. Diese Anforderung ermöglicht es Forschern, Forschungsdaten bei Vorliegen einer entsprechenden Einwilligung aus verschiedenen Forschungsprojekten für einen Probanden zusammenzuführen.

\subsubsection{Qualitätsanforderungen}

Die sieben identifizierten Qualitätsanforderungen in Tabelle 14 sind die zentralen Qualitätsmerkmale des Probandenmanagementsystems.

Wichtig ist, dass das Probandenmanagementsystem für die Benutzer verständlich und nachvollziehbar gestaltet ist (A-Q-1). So sollen Aktionen, die der Benutzer ausführt, keine für ihn unvorhersehbaren Ergebnisse hervorrufen. Die Benutzeroberfläche soll daher für die Benutzer einfach zu bedienen sein (A-Q-2). Benutzer sollen sich direkt im System zurecht finden und keine umfangreiche Einweisung benötigen. 
Da das Probandenmanagementsystem in verschiedenen Forschungsprojekten, auch klinischen Studien, der Universitätsmedizin Göttingen eingesetzt werden soll, müssen auch deren zugrunde liegenden Regularien berücksichtigt werden. Dies wären die international anerkannten Regularien der Good Clinical Practice [155] zur Qualitätssicherung von klinischen Studien (AQ-3) sowie die Good Automated Manufacturing Practice [156, 157], welche als Leitfaden zur Validierung von computergestützten Systemen fungiert (A-Q-4).

Des Weiteren soll das System die Datenintegration sicherstellen (A-Q-5). Es soll so gestaltet sein, dass einmal erfasste Datensätze nicht nochmals erfasst werden müssen [155].

\begin{tabular}{l|l|l} 
Nr. & Beschreibung & Quelle \\
\hline A-Q-1 & $\begin{array}{l}\text { Das System soll verständlich und nachvollziehbar für den Be- } \\
\text { nutzer sein. }\end{array}$ & {$[99]$} \\
\hline A-Q-2 & $\begin{array}{l}\text { Die Benutzeroberfläche des Systems soll für den Benutzer } \\
\text { einfach zu bedienen sein. }\end{array}$ & {$[99]$} \\
\hline A-Q-3 & $\begin{array}{l}\text { Das System soll den Regularien der Good Clinical Practice } \\
\text { (GCP) entsprechen. }\end{array}$ & I-9 \\
\hline A-Q-4 & $\begin{array}{l}\text { Das System soll den Regularien der Good Automated Manu- } \\
\text { facturing Practice (GAMP) entsprechen. }\end{array}$ & I-9 \\
\hline A-Q-5 & $\begin{array}{l}\text { Das System soll so gestaltet sein, dass einmal erfasste Datens- } \\
\text { ätze nicht erneut erfasst werden müssen. }\end{array}$ & {$[71,155]$} \\
\hline A-Q-6 & $\begin{array}{l}\text { Die Softwarearchitektur des Systems soll so gestaltet sein, } \\
\text { dass sie einfach erweiterbar ist. }\end{array}$ & $\begin{array}{l}{[109,119,120,} \\
128]\end{array}$ \\
\hline A-Q-7 & $\begin{array}{l}\text { Die Softwarearchitektur des Systems soll nachvollziehbar ge- } \\
\text { staltet sein. }\end{array}$ & {$[119,120,128]$}
\end{tabular}

Tabelle 14. Lösungsorientierte Qualitätsanforderungen

Die beiden verbleibenden Qualitätsanforderungen beziehen sich auf die Softwarearchitektur des Probandenmanagementsystems. Diese soll so gestaltet sein, dass sie zum einen einfach erweiterbar ist (A-Q-6) und zum anderen nachvollziehbar gestaltet ist (A-Q-7). Durch eine erweiterbare und nachvollziehbare Softwarearchitektur können neue in Zukunft benötigte Funktionen mit geringem Aufwand in das System integriert werden [119, 120].

\subsubsection{Randbedingungen}

Insgesamt wurden neun Randbedingungen identifiziert (siehe Tabelle 15). Das Probandenmanagementsystem soll als Webanwendung konzipiert werden (A-R-1); ein Systemzugriff soll nach Möglichkeit mit allen gängigen Webbrowsern möglich sein. Eine Installation weiterer Software, wie z. B. Flash oder Java, soll nicht notwendig sein (A-R-2). Diese Randbedingungen rühren daher, dass PC-Arbeitsplätze an der Universitätsmedizin Göttingen umfangreichen Restriktionen unterliegen. So können beispielsweise Softwareanwendungen nicht von den Benutzern installiert werden, da dies immer durch einen Administrator erfolgen muss. Webanwen- 
dungen bleiben von diesen Restriktionen größtenteils unberührt, solange keine weitere Software (Flash, Java etc.) benötigt wird. Darüber hinaus soll das Probandenmanagementsystem mehrsprachig ausgelegt sein (A-R-3).

Alle getätigten Aktionen, wie das Anlegen, Bearbeiten oder Löschen eines Datensatzes, sollen durch das System dokumentiert werden (A-R-4). So ist es beispielsweise möglich, alle Änderungen an einem Datensatz auch nach vielen Jahren immer noch nachzuvollziehen.

\begin{tabular}{l|l|l} 
Nr. & Beschreibung & Quelle \\
\hline A-R-1 & Das System soll als Webanwendung konzipiert sein. & I-6, [1, 153] \\
\hline A-R-2 & $\begin{array}{l}\text { Die Webanwendung des Systems soll ohne weitere Software, } \\
\text { wie z. B. Flash oder Java, auskommen. }\end{array}$ & I-6, [1] \\
\hline A-R-3 & Das System soll unterschiedliche Sprachen unterstützen. & {$[1]$} \\
\hline A-R-4 & $\begin{array}{l}\text { Alle Aktionen, die an den erfassten Datensätzen durch die Be- } \\
\text { nutzer vorgenommen werden, sollen dokumentiert werden. }\end{array}$ & I-8, [1, 90] \\
\hline A-R-5 & $\begin{array}{l}\text { Das System soll konform mit den Regularien des Niedersäch- } \\
\text { sischen Datenschutzgesetzes sein. }\end{array}$ & I-3, I-11 \\
\hline A-R-6 & $\begin{array}{l}\text { Das System soll konform mit den Empfehlungen des generi- } \\
\text { schen Datenschutzkonzepts der TMF sein. }\end{array}$ & I-3, I-11, I-12 \\
\hline A-R-7 & $\begin{array}{l}\text { Das System soll in die IT-Infrastruktur der medizinischen For- } \\
\text { schungsprojekte integrierbar sein. }\end{array}$ & [1] \\
\hline A-R-8 & $\begin{array}{l}\text { Das System soll in die IT-Infrastruktur der Universitätsmedi- } \\
\text { zin Göttingen integrierbar sein. }\end{array}$ & I-6, I-9, [1] \\
\hline A-R-9 & $\begin{array}{l}\text { Der Betrieb des Systems soll im gesicherten Intranet der Uni- } \\
\text { versitätsmedizin Göttingen erfolgen. }\end{array}$ & I-3
\end{tabular}

Tabelle 15. Lösungsorientierte Randbedingungen

Das Probandenmanagementsystem bzw. dessen Betrieb an der Universitätsmedizin Göttingen, fällt unter den Anwendungsbereich des Niedersächsischen Datenschutzgesetzes (A-R-5). Besonders relevant ist §25 NDSG, welcher die Regelungen zur Verarbeitung personenbezogener Daten für Forschungsvorhaben umfasst. Wesentlich für das System (A-R-6) sind auch die Empfehlungen des generischen Datenschutzkonzepts der TMF [47]. Das Probandenmanagementsystem soll sowohl in die IT-Infrastrukturen der Forschungsprojekte (A-R-7), als auch in die IT-Infrastruktur der Universitätsmedizin Göttingen (A-R-8) integrierbar sein.

Die Zuordnung der Probanden zu den jeweiligen Forschungsprojekten enthält Informationen über deren Gesundheitszustand. So kann aus der Tatsache, dass ein Proband an einem Forschungsprojekt für seltene Erkrankungen teilnimmt, geschlossen werden, dass er an eben dieser Erkrankung leidet. Der Betrieb des Probandenmanagementsystems muss nach Rücksprache mit dem Datenschutzbeauftragten der Universitätsmedizin Göttingen daher im gesicherten Intranet der Universitätsmedizin Göttingen erfolgen (A-R-9). 


\section{Architekturkonzept}

Die Entwicklung des Architekturkonzepts für das Probandenmanagementsystem erfolgte durch Anwendung der Methodik des Domain-Driven Design. Folglich umfasst das Architekturkonzept ein Domänenmodell (Abschnitt 4.1) sowie primäre und sekundäre Schnittstellen (Abschnitt 4.2) in Form von UML-Klassendiagrammen.

Zur besseren Übersicht wurden die jeweiligen Klassendiagramme des Domänenmodells in Abschnitt 4.1 in sieben Bereiche unterteilt: Systemkonfiguration, Sicherheit, Projektverwaltung, Probandenverwaltung, Forschungsakte, Kommunikation und Pseudonymisierung.

Die Klassendiagramme zur Verwaltung von Einwilligungserklärungen (A-F-20 bis A-F-24) und zur Generierung von projektübergreifenden Forschungspseudonymen (A-F-30) finden sich in den Abschlussarbeiten von Markus Matoni [118] und Martin Schwarzmaier [117]. Aufgrund des umfangreichen Forschungsfeldes der beiden Themenbereiche wurde auf eine Einarbeitung in diese Dissertation verzichtet. Daher werden die zugehörigen Anforderungen (A-F-20 bis AF-24 und A-F-30) nachfolgend nicht weiterbetrachtet und folgerichtig in dieser Dissertation als nicht erfüllt angesehen.

Insgesamt werden somit 24 der 30 identifizierten lösungsorientierten Anforderungen durch das entwickelte Architekturkonzept berücksichtigt. Durch die Verwendung des Architekturstils Ports and Adapters werden die Erweiterbarkeit (A-Q-6) und die Nachvollziehbarkeit (A-Q-7) des Architekturkonzepts sichergestellt.

\subsection{Domänenmodell}

Das Domänenmodell besteht gemäß dem Architekturstil Ports and Adapters aus Entities, Aggregates und Value Objects, die in den UML-Diagrammen durch Klassen repräsentiert werden. Alle modellierten Entities erben die Eigenschaften von der abstrakten Klasse AbstractPersistable. Diese umfasst einen Identifikator und kennzeichnet die erbende Klasse, welche zur Speicherung in einer Datenbank vorgesehen ist. Die Vererbung und die Klasse AbstractPersistable werden nur in Abbildung 17 exemplarisch dargestellt und zur besseren Übersicht in den nachfolgenden Klassendiagrammen weggelassen.

\subsubsection{Systemkonfiguration}

Der Bereich Systemkonfiguration umfasst neben der übergeordneten Klasse AbstractPersistable die beiden zentralen Klassen Report und Code (siehe Abbildung 17).

Ein Report ermöglicht die Aggregation der erfassten Daten im Probandenmanagementsystem. Er kann beispielsweise für Exporte von Probandenstammdaten (A-F-19) verwendet werden. Reports beinhalten eine Datenbankabfrage (query), die bei Erstellung des Reports ausgeführt wird. Die Zuordnung eines Reports zu genau einem Forschungsprojekt ist bei projektübergreifenden Reports nicht möglich. Daher werden Reports nicht Forschungsprojekten, sondern UserAccounts, also Benutzern des Probandenmanagementsystems, zugeordnet. 


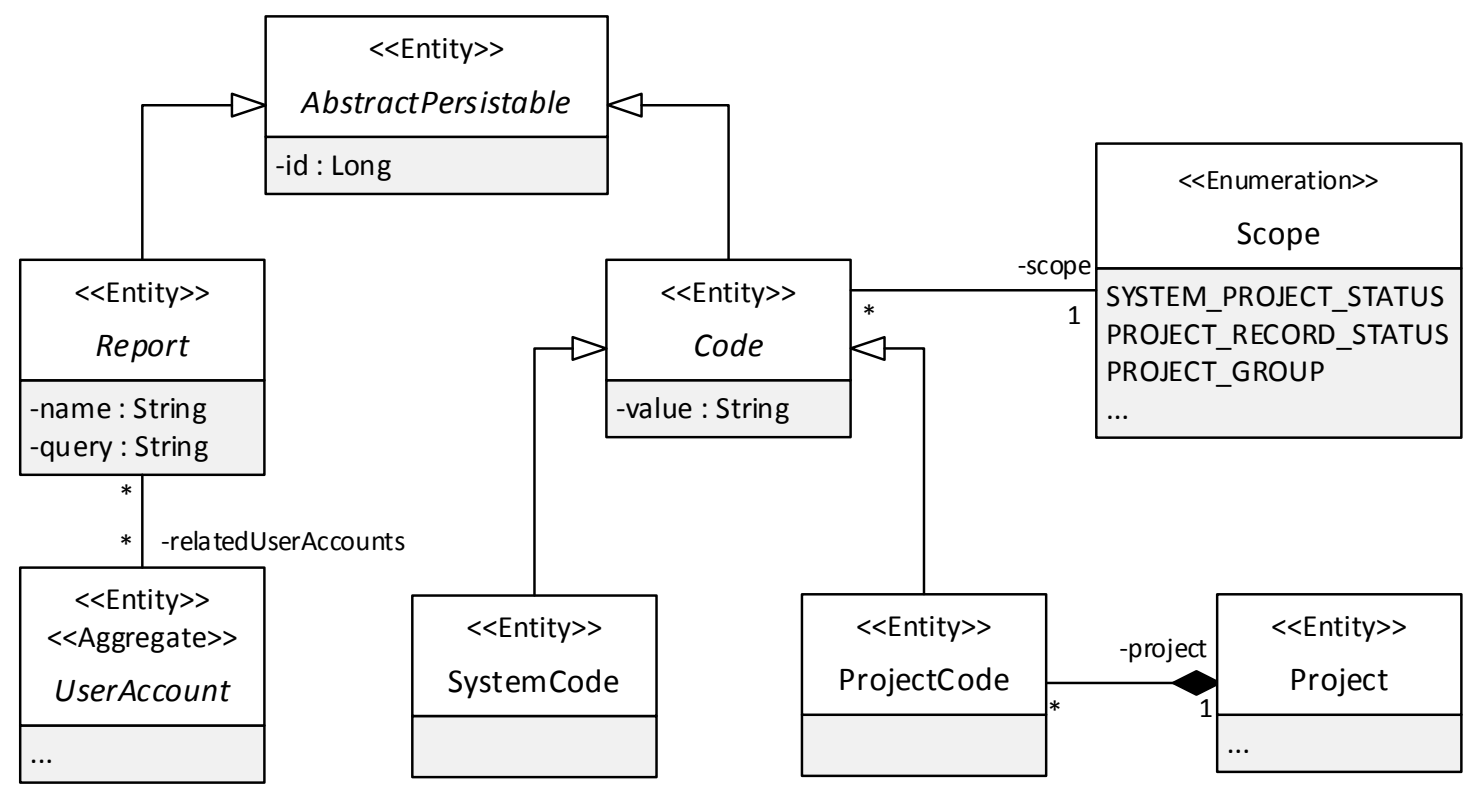

Abbildung 17. Domänenmodell des Bereichs Systemkonfiguration

Die Klasse Code ermöglicht die Erstellung von domänenspezifischen Kodierungen für spezielle Bereiche innerhalb des Probandenmanagementsystems. Ein Bereich wird durch einen Scope festgelegt, dieser kann systemweit gültig sein oder nur für ein einzelnes Forschungsprojekt. Dies wird durch die beiden Klassen SystemCode und ProjectCode repräsentiert. Ein ProjectCode ist immer einem Project (Forschungsprojekt) zugeordnet. Codes werden beispielsweise im Architekturkonzept zur Beschreibung des Status eines Forschungsprojekts im System verwendet. Durch die Verwendung von Codes ist das Probandenmanagementsystem einfach an spezifische Anforderungen anzupassen.

\subsubsection{Sicherheit}

Kern des Bereichs Sicherheit (siehe Abbildung 18) bildet die Klasse UserAccount. Ein UserAccount repräsentiert einen Benutzer des Probandenmanagementsystems. UserAccount ist eine abstrakte Oberklasse mit den beiden Unterklassen ADUserAccount und LocalUserAccount. Die Klasse ADUserAccount ermöglicht die Authentifizierung eines Benutzers durch die zentrale Benutzerverwaltung der Universitätsmedizin Göttingen (A-F-1). Dies erfolgt durch den Windows Verzeichnisdienst Active Directory (AD) und basiert grundlegend auf dem Lightweight Directory Access Protocol [158]. Die Authentifizierung innerhalb des Probandenmanagementsystems (A-F-2) erfolgt durch den LocalUserAccount.

Die Zuordnung von Benutzern zu Forschungsprojekten wird durch die Klasse ProjectAccess sichergestellt. ProjectAccess verweist immer auf genau einen UserAccount und ein Project. Hierdurch kann ein Benutzer mehreren Forschungsprojekten zugeordnet werden (A-F-7). Ebenso ermöglicht dies den Benutzern eine Übersicht über alle Forschungsprojekte, denen sie zugeordnet sind (A-F-8). Neben UserAccount und Project verweist ProjectAccess zudem noch auf die Klasse Role, welche die Rolle des Benutzers im Forschungsprojekt abbildet. 


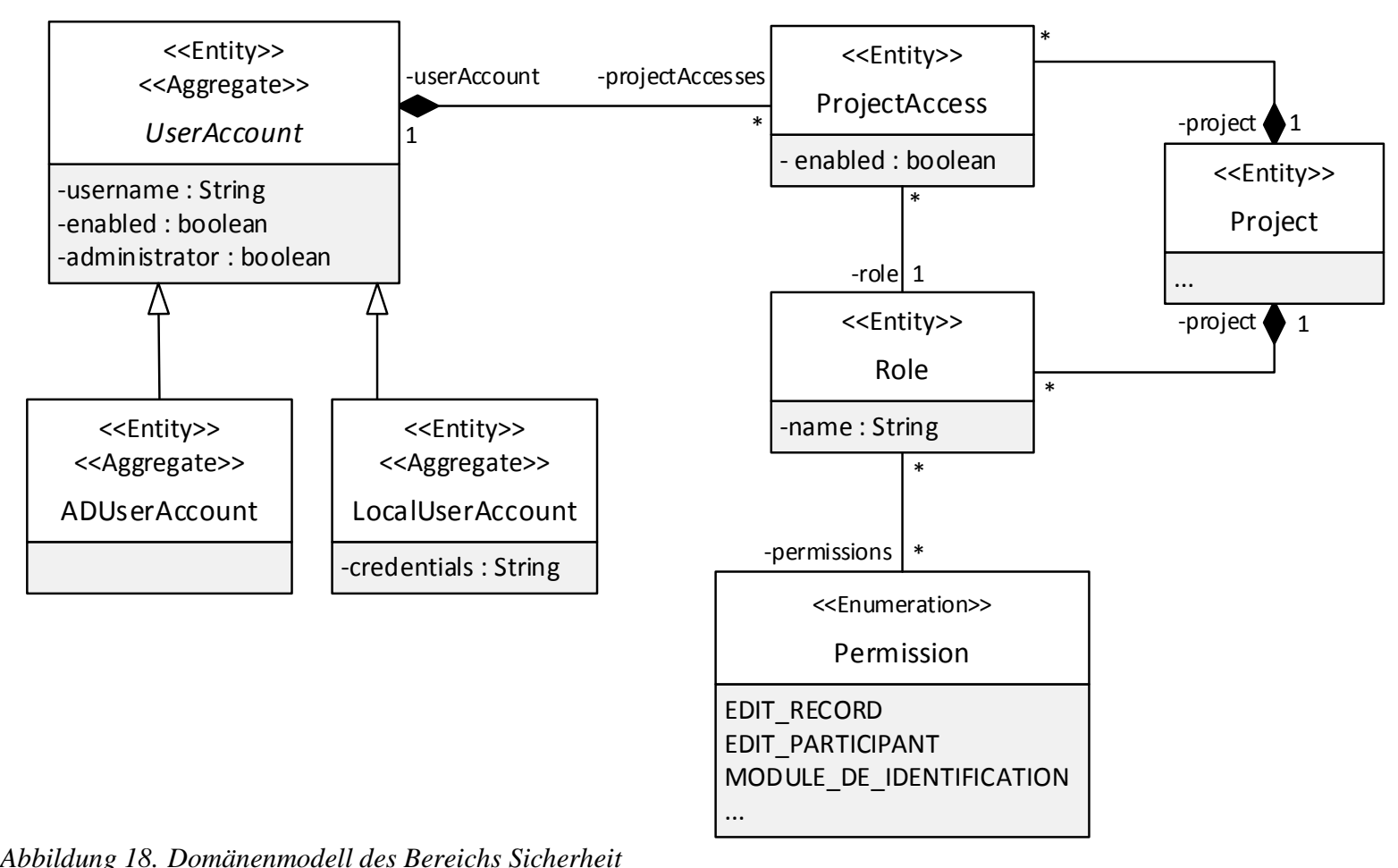

Abbildung 18. Domänenmodell des Bereichs Sicherheit

Role bildet den Ausgangspunkt für das feingranulare Rechte- und Rollenmanagement (A-F-3) des Probandenmanagementsystems. Die Rollen werden für jedes Forschungsprojekt einzeln konfiguriert; dies erfolgt durch die Zuweisung von Permissions zu einer Role. Permissions bilden die Berechtigungen im Probandenmanagementsystem ab, beispielsweise zur Änderung von Forschungsakten oder Probandenstammdaten. Durch das Rechte- und Rollenmanagement kann beispielsweise die Änderung von Probandenstammdaten nur für Administratoren festgelegt werden (A-F-11).

\subsubsection{Projektverwaltung}

Innerhalb des Domänenmodells repräsentiert die Klasse Project ein reales medizinisches Forschungsprojekt an der Universitätsmedizin Göttingen (siehe Abbildung 19). Generell ermöglicht das Probandenmanagementsystem die Erfassung und Verwaltung einer beliebigen Anzahl von Forschungsprojekten.

Jedes Project verweist auf einen Code, der wiederum den Status des Forschungsprojekts im System darstellt. Des Weiteren wird die Klasse Code verwendet um den Typ für ein Forschungssystem (ResearchSystem) zu hinterlegen, z. B. als Studiendatenbank oder Biomaterialdatenbank. Die Klasse ResearchSystem verweist auf das zugehörige Project, sodass neben dem Projektstatus auch die Forschungssysteme zu einem Forschungsprojekt erfasst werden können (A-F-5). 


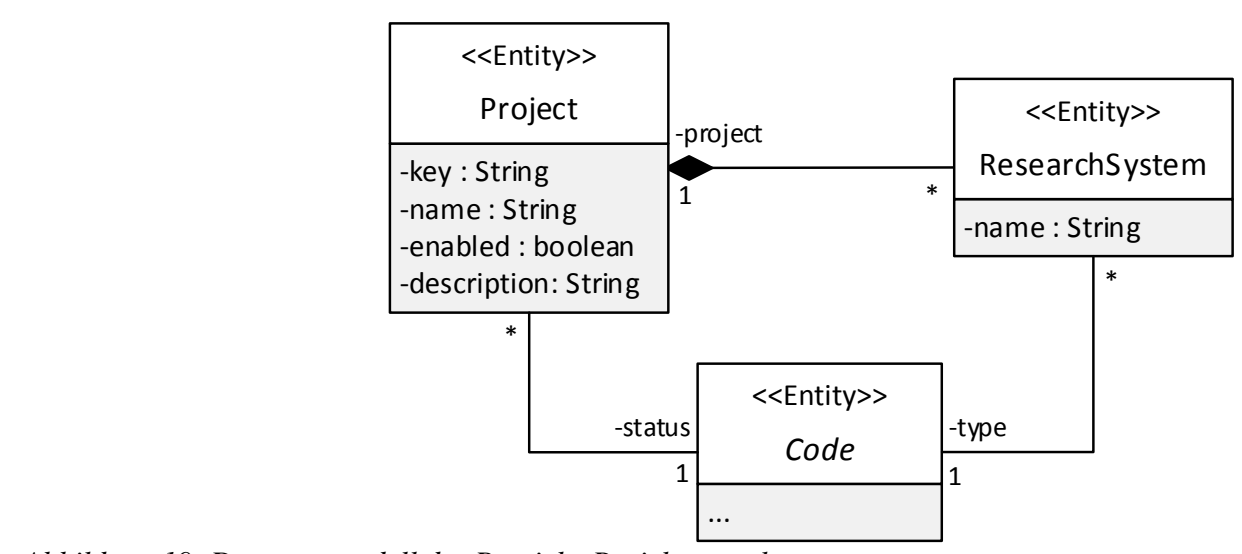

Abbildung 19. Domänenmodell des Bereichs Projektverwaltung

\subsubsection{Probandenverwaltung}

Der Proband wird durch die Klasse Participant repräsentiert (siehe Abbildung 20). Diese umfasst alle geforderten Stammdaten eines Probanden (A-F-9).

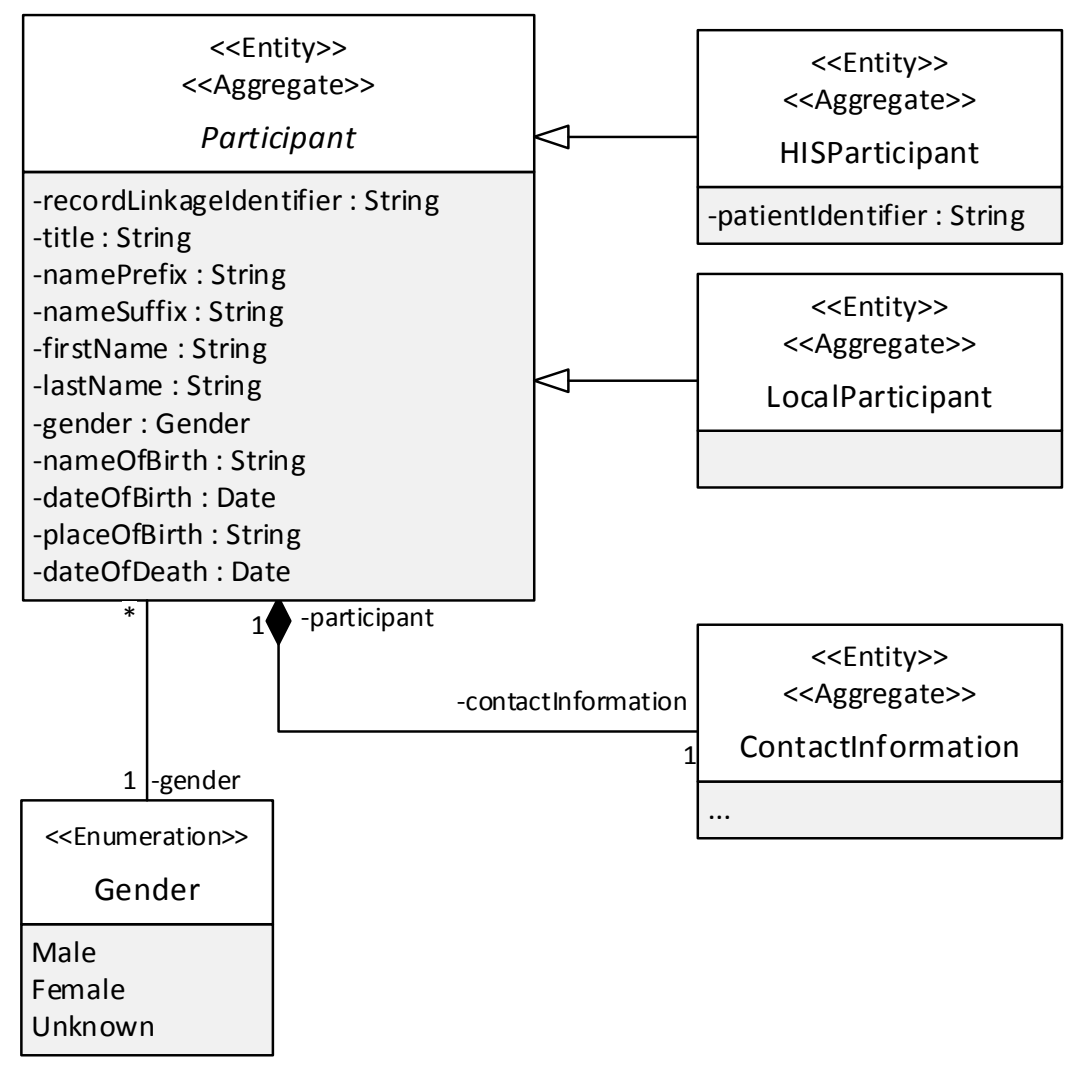

Abbildung 20. Domänenmodell des Bereichs Probandenverwaltung

Innerhalb des Domänenmodells existieren zwei Ausprägungen von Probanden, die anhand der Quelle ihrer Stammdaten unterschieden werden. Die Probandenstammdaten der ersten Ausprägung (HISParticipant) entstammen in Göttingen SAP IS-H (A-F-13), in anderen Einrichtungen kann auch jedes andere klinisch-administrative System als Quelle genutzt werden. Die zweite Ausprägung (LocalParticipant) bildet die Anforderung ab, dass das Anlegen eines Probanden mittels direkter Eingabe seiner Stammdaten möglich sein muss (A-F-15). Zur besseren Über- 
sicht wurden die Kontaktinformationen der Probanden (ContactInformation) im Klassendiagramm verkürzt dargestellt. In Anhang A.6 findet sich die vollständige Darstellung der Klasse ContactInformation.

Die zugehörige primäre Schnittstelle des Bereichs Probandenverwaltung (ParticipantService, siehe Anhang A.6) stellt sicher, dass eine Änderung von Stammdaten nur möglich ist, wenn ein Proband durch die Klasse LocalParticipant repräsentiert wird. Dies ist notwendig, da die Stammdaten von HISParticipant extern verwaltet werden und eine Änderung im Probandenmanagementsystem daher nicht zielführend ist.

\subsubsection{Forschungsakte}

Das Bindeglied zwischen Forschungsprojekten und deren Probanden bildet die Klasse Record (Forschungsakte). Sie repräsentiert die Teilnahme eines Probanden an einem Forschungsprojekt (siehe Abbildung 21).

Vergleichbar ist dieser Ansatz mit dem Konzept der Fallakte im Krankenhaus: Patientenakten im Krankenhaus werden oftmals in einzelne Aufenthaltsfälle unterteilt, die eine Behandlung bzw. einen Krankenhausaufenthalt des Patienten abbilden [71].

In Verbindung mit einer Forschungsakte können alle relevanten Angaben über einen Probanden für ein Forschungsprojekt erfasst werden. Dies wäre beispielsweise der Status (repräsentiert durch einen Code) oder das Rekrutierungsdatum (A-F-18).

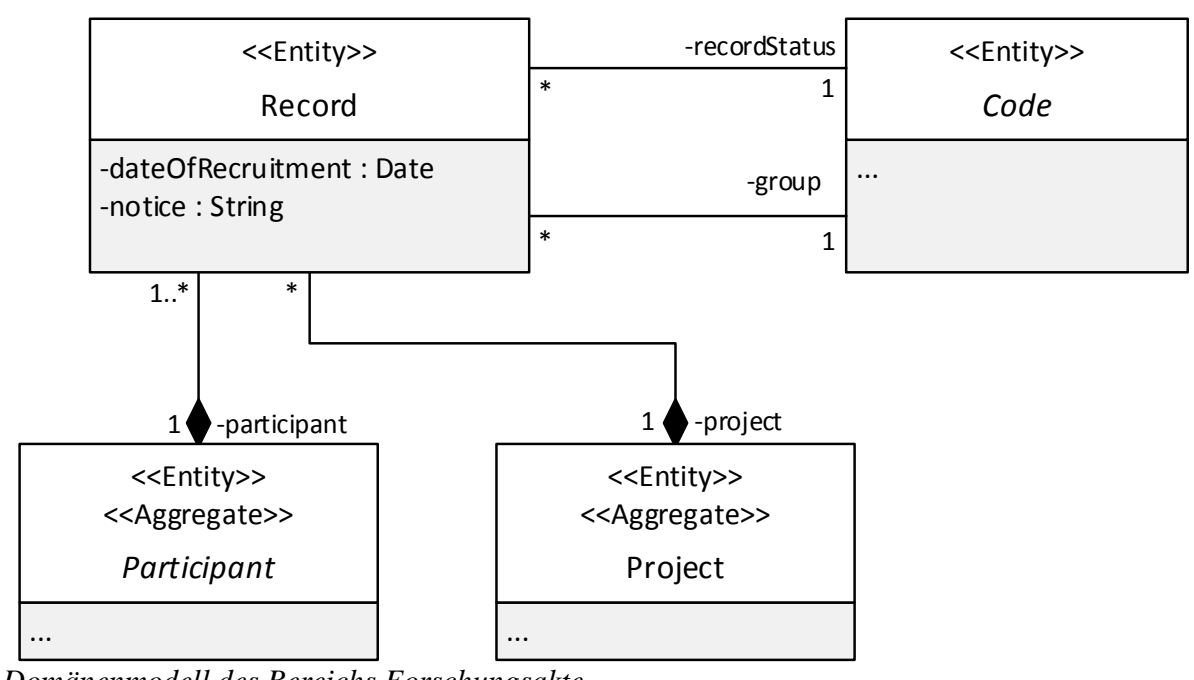

Abbildung 21. Domänenmodell des Bereichs Forschungsakte

Die Klasse Record verweist immer auf einen Participant und ein Project. Hierdurch wird die projektübergreifende Verwaltung von Probanden sowie deren Teilnahme an mehreren Forschungsprojekten ermöglicht (A-F-10). Zudem wird so gewährleistet, dass ein vorhandener Proband einem neuen Forschungsprojekt zugewiesen werden kann (A-F-14 und A-Q-5). Über den Verweis auf Project und somit auf ProjectAccess, steht eine Übersicht über alle Probanden eines Benutzers zur Verfügung (A-F-17). 
Eine vollständige Löschung eines Probanden aus dem System kann nur erfolgen, wenn alle zugeordneten Forschungsakten ebenfalls gelöscht werden. Zieht ein Proband die Einwilligung zu einem Forschungsprojekt zurück, wird nur die betreffende Forschungsakte aus dem System entfernt (A-F-12), sodass der Proband gegebenenfalls in anderen Projekten weitergeführt werden kann.

\subsubsection{Kommunikation}

Die medizinische Forschung in Deutschland fördert die Vernetzung zwischen einzelnen Forschungseinrichtungen [36, 37, 49]. Ein typischer Anwendungsfall in diesem Zusammenhang ist die Nutzung einer zentralen Softwareanwendung zur Pseudonymisierung in Verbundforschungsprojekten [47].

Innerhalb des Bereichs Kommunikation wird die Vernetzung des Probandenmanagementsystems mit externen Systemen der Verbundforschung am Beispiel Pseudonymisierung modelliert (siehe Abbildung 22). Ein externes System zur Pseudonymisierung wird durch die Klassen ExternalSystem repräsentiert. Diese wiederum ist immer einem Project zugeordnet, wodurch Forschungsprojekte mehrere externe Systeme umfassen können.

Die Klasse ExternalSystem ist eine abstrakte Oberklasse, deren Unterklassen spezifische Softwareanwendungen zur Pseudonymisierung darstellen. Beispielhaft wurden drei Softwareanwendungen modelliert: PIDGenerator [43], PIDDispatcher [2] und Mainzelliste [86].

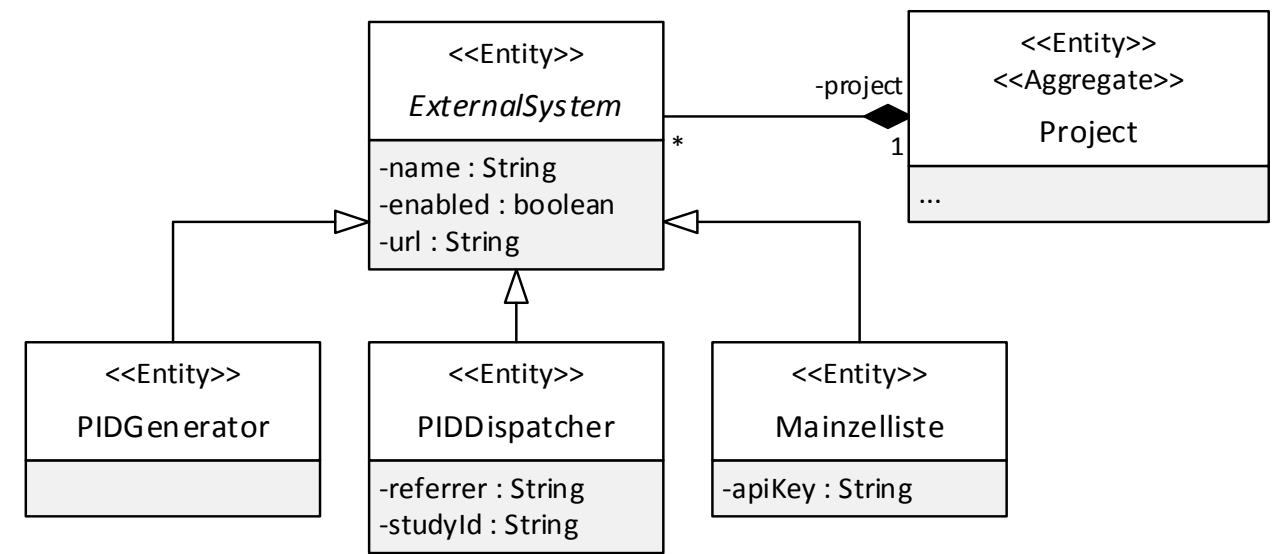

Abbildung 22. Domänenmodell des Bereichs Kommunikation

\subsubsection{Pseudonymisierung}

Kern des Bereichs Pseudonymisierung ist die Klasse Identifier, die das Pseudonym eines Probanden in einem Forschungsprojekt darstellt. Daher ist ein Identifier immer genau einem $R e$ cord zugeordnet (siehe Abbildung 23). Diese Zuordnung ermöglicht die Suche nach Probanden anhand ihrer Pseudonyme (A-F-27).

In der medizinischen Forschung dienen Pseudonyme zur Trennung der Probandenidentität (z. B. Stammdaten) von den Forschungsdaten [42]. Die Erfassung der Forschungsdaten erfolgt beispielsweise in einer Studiendatenbank oder Biomaterialverwaltung. Einem Identifier können 
daher verschiedene Forschungssysteme in Form der Klasse ResearchSystem zugeordnet werden. Hierdurch kann die Verwendung eines Pseudonyms in einem Forschungssystem dokumentiert werden (A-F-26).

Die Klasse Identifier ist eine abstrakte Oberklasse und besitzt die drei Unterklassen GeneratedIdentifier, ManualIdentifier und ExternalIdentifier. Mittels GeneratedIdentifier wird die interne Generierung von Pseudonymen durch einen Pseudonymgenerator (IdentifierGenerator) ermöglicht (A-F-28). Beispielhaft wurde die Klasse RandomIdentifierGenerator modelliert, welche die Generierung eines zufälligen Pseudonyms anhand von Parametern ermöglicht.

ManualIdentifier stellt innerhalb des Probandenmanagementsystems sicher, dass Pseudonyme auch manuell erstellt und erfasst werden können (A-F-25).

Eng mit dem Bereich Kommunikation ist die Unterklasse ExternalIdentifier verbunden. Diese Klasse repräsentiert ein Pseudonym, welches aus einer externen Softwareanwendung zur Pseudonymisierung abgerufen wurde (A-F-29).

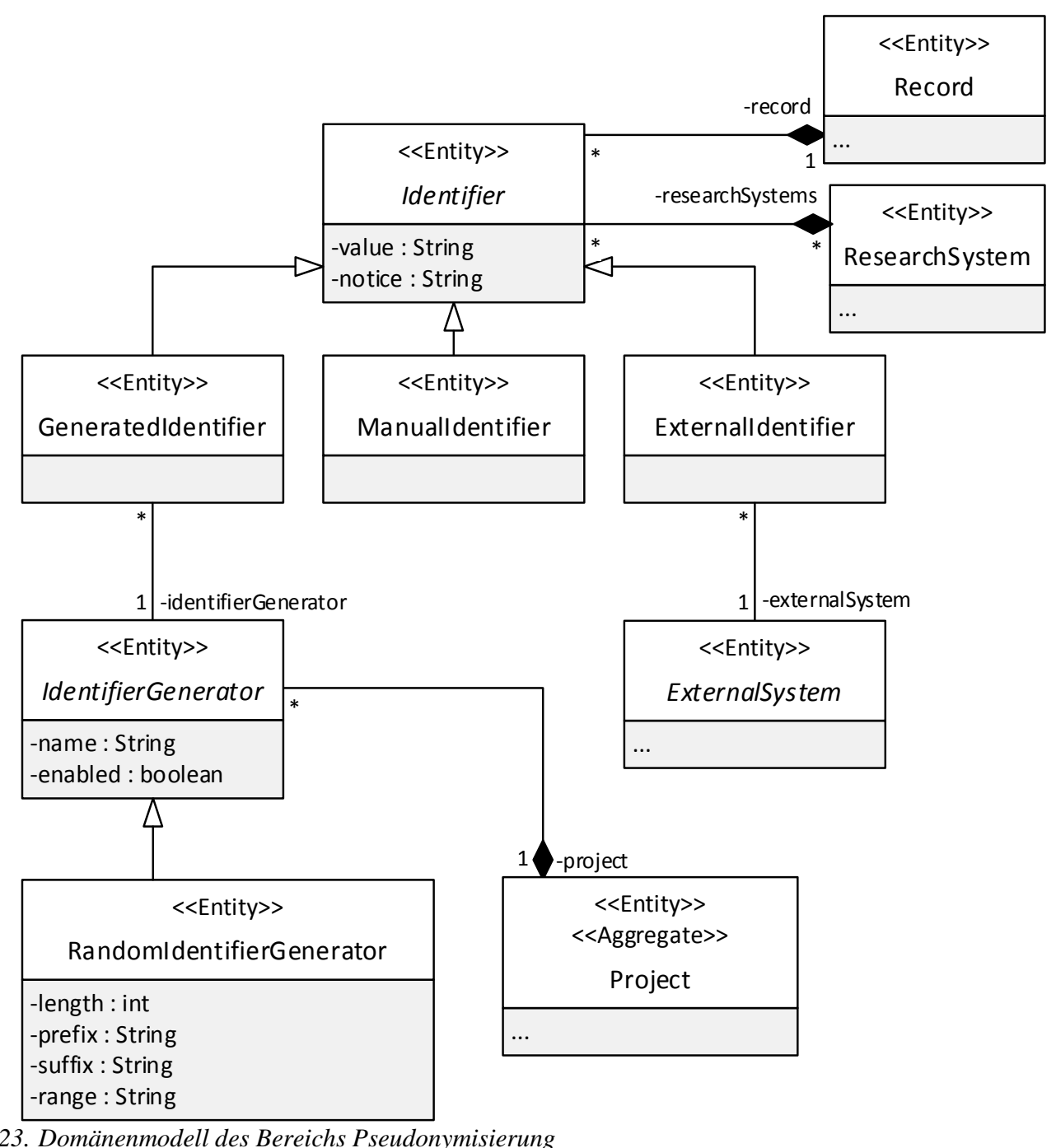




\subsection{Primäre und sekundäre Schnittstellen}

Insgesamt umfasst das Architekturkonzept neun primäre sowie 13 sekundäre Schnittstellen. Die sekundären Schnittstellen unterteilen sich in neun Repositories und vier Provider. Da die primären Schnittstellen und die Repositories ähnliche Funktionen aufweisen, findet sich eine vollständige Darstellung der Klassen in Anhang A.6. In ihrer Gesamtheit ermöglichen die primären und sekundären Schnittstellen den Import und Export von Probandenstammdaten (A-F-19).

Kern dieses Abschnitts bilden die vier modellierten Provider sowie deren neun Implementierungen in Form von Adaptern. Die Gliederung erfolgt gemäß den Bereichen: Probandenverwaltung, Kommunikation und Pseudonymisierung.

\subsubsection{Probandenverwaltung}

Der Bereich Probandenverwaltung umfasst neben dem ParticipantRepository die sekundären Schnittstellen RecordLinkageProvider (siehe Abbildung 24) und HISProvider (siehe Abbildung 25).

Primäre Aufgabe des RecordLinkageProvider ist die Bereitstellung einer Duplikaterkennung, sodass identische Probanden nicht mehrfach im System angelegt werden (A-F-16). Der RecordLinkageProvider umfasst eine Funktion zur Erzeugung eines Identifikators anhand der Probandenstammdaten sowie des Forschungsprojekts. Probanden mit den gleichen Stammdaten sollen einen identischen Identifikator erhalten. Des Weiteren umfasst der RecordLinkageProvider eine Funktion zur Verifikation des existierenden Identifikators, sowie zu dessen Löschung.

Abbildung 24. RecordLinkageProvider des Bereichs Probandenverwaltung

Innerhalb des Architekturkonzepts sind drei Implementierungen des RecordLinkageProvider vorgesehen. Die erste Implementierung bildet der MockRecordLinkageAdpater. Dieser ist zur Testung bzw. für die Entwicklung gedacht und generiert bei jedem Aufruf, unabhängig von den Parametern, einen neuen Identifikator. Es wird keine Duplikaterkennung durchgeführt. Anders 
verhält es sich bei dem PIDGeneratorRecordLinkageAdpater. Dieser nutzt die Softwareanwendung PIDGenerator zur Erkennung von ähnlichen Probanden anhand des Vornamens, Nachnamens, Geburtsdatums und Geschlechts [43]. Die letzte Implementierung bildet der ProjectRecordLinkageAdapter, dieser ermöglicht ebenfalls eine Duplikaterkennung anhand des Vornamens, Nachnamens, Geburtsdatums und Geschlechts. Im Vergleich zu den beiden anderen Implementierungen berücksichtigt der ProjectRecordLinkageAdapter jedoch das jeweilige Forschungsprojekt des Probanden. Diese Implementierung ermöglicht es, die projektübergreifende Verwaltung von Probanden zu deaktivieren, wenn beispielsweise keine entsprechende Einwilligung der Probanden für eine übergreifende Verwaltung vorhanden ist oder diese nicht gewünscht ist.

Die Anbindung des Probandenmanagementsystems an das Krankenhausinformationssystem erfolgt über den HISProvider (siehe Abbildung 25). Dieser dient zum Abruf von Stammdaten aus dem klinisch-administrativen System; an der Universitätsmedizin Göttingen ist dies SAP IS-H (A-F-13). Die Suche nach Krankenhauspatienten erfolgt anhand der Patientenkennung oder Fallnummer. Fallnummern sind immer einer Patientenkennung zugeordnet, diese wiederum bildet den primären Identifikator des Patienten im Krankenhaus.

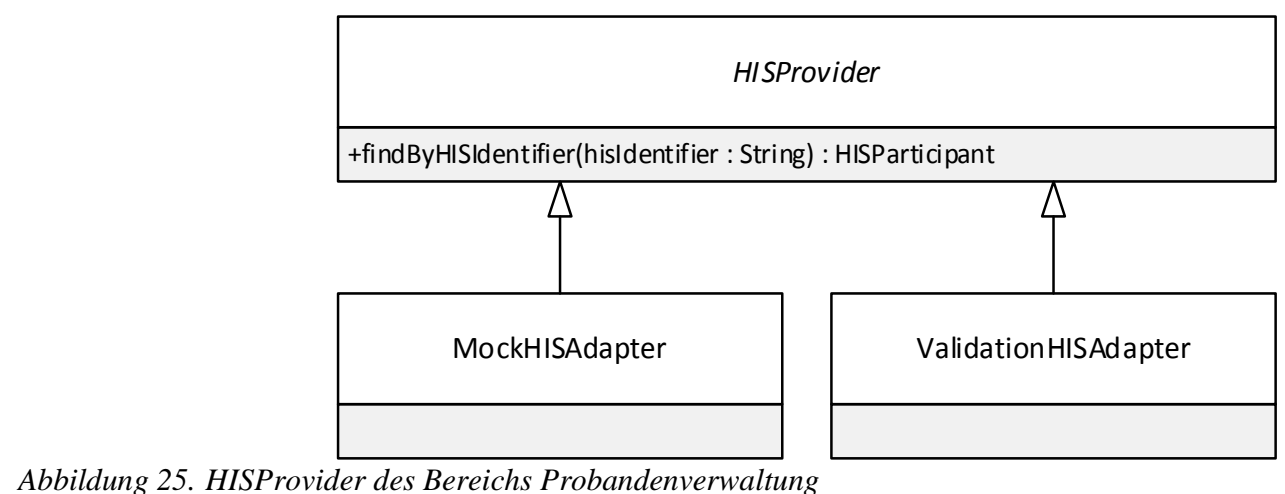

Abbildung 25. HISProvider des Bereichs Probandenverwaltung

Im Architekturkonzept sind zwei Implementierungen des HISProvider vorgesehen. Der MockHISApdater dient zur Testung bzw. Entwicklung und generiert bei jedem Aufruf eine leere Ergebnismenge. Für die Validierung des Probandenmanagementsystems wurde eine spezielle Implementierung (ValidationHISAdapter) vorgenommen, die bei Übergabe eines vorgegebenen Identifikators einen Krankenhauspatienten generiert. Eine konkrete Implementierung für die Anbindung an das SAP IS-H wurde im Architekturkonzept auf Grund des hohen Implementierungsaufwands nicht vorgesehen. Diese würde jedoch grundlegend auf der vorhandenen sekundären Schnittstelle des HISProvider basieren.

\subsubsection{Kommunikation}

Kern des Bereichs Kommunikation bildet die sekundäre Schnittstelle ExternalSystemProvider (siehe Abbildung 26). Diese repräsentiert ein externes System zur Pseudonymisierung von Probanden. 
Derzeit verfügt ExternalSystemProvider über eine Funktion zum Abruf eines Pseudonyms anhand der Forschungsakte des Probanden. Weitere Parameter dieser Funktion sind die Konfiguration des externen Systems und der Benutzer, welcher die Pseudonymisierung vornimmt. Ziel der sekundären Schnittstelle ExternalSystemProvider ist es eine einheitliche Kommunikation mit externen Systemen im Bereich der Pseudonymisierung zu ermöglichen. Das Architekturkonzept umfasst daher drei konkrete Implementierungen der sekundären Schnittstelle, welche jeweils eine Softwareanwendung repräsentieren.

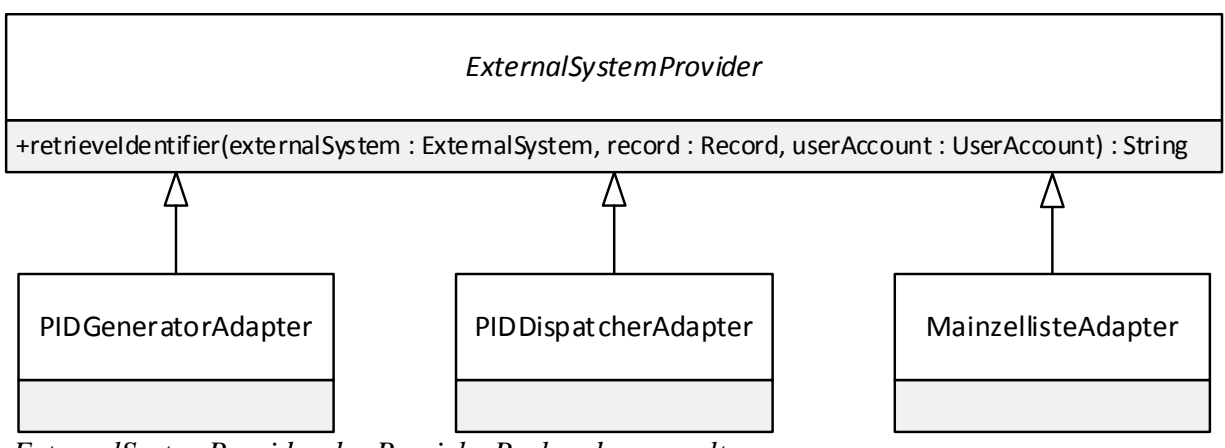

Abbildung 26. ExternalSystemProvider des Bereichs Probandenverwaltung

Der PIDGeneratorAdapter ermöglicht die Pseudonymisierung eines Probanden über die Softwareanwendung PIDGenerator [43]. Wie auch die Mainzelliste ist der PIDGenerator frei verfügbar und wurde daher als Adapter in das Architekturkonzept aufgenommen. Der PIDDispatcher ist eine kommerzielle Software. Er wird vornehmlich in den Forschungsprojekten des Instituts für Medizinische Informatik der Universitätsmedizin Göttingen eingesetzt und wurde daher im Architekturkonzept berücksichtigt. Die letzte Implementierung bildet die Mainzelliste [86]. Da der Quellcode ebenfalls frei verfügbar ist, wurde sie als dritte Softwareanwendung in Form des MainzellisteAdapter in das Architekturkonzept aufgenommen.

Die drei Softwareanwendungen wurden beispielhaft im Architekturkonzept berücksichtigt. Es ist durchaus ohne eine grundlegende Änderung der sekundären Schnittstelle (PIDGeneratorAdapter) möglich, weitere Softwareanwendungen durch entsprechende Adapter anzubinden.

\subsubsection{Pseudonymisierung}

Der Bereich Pseudonymisierung umfasst neben den Repositories eine sekundäre Schnittstelle zur Generierung von Pseudonymen (siehe Abbildung 27).

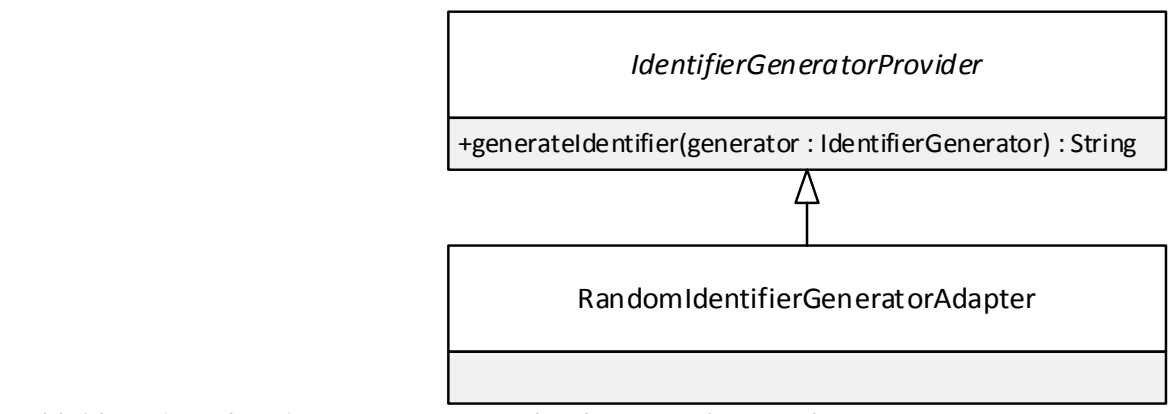

Abbildung 27. IdentifierGeneratorProvider des Bereichs Pseudonymisierung 
Diese wird durch den IdentifierGeneratorProvider repräsentiert. Entsprechend der Generatorkonfiguration wird durch den Generator ein Pseudonym erzeugt. Im Architekturkonzept wurde eine konkrete Umsetzung des IdentifierGeneratorProvider in Form des RandomIdentifierGeneratorAdapter berücksichtigt. Dieser generiert ein Pseudonym auf Grundlage einer zufälligen Abfolge von Zahlen und Buchstaben. Prinzipiell ist es möglich das Probandenmanagementsystem um weitere Generatoren für Pseudonyme zu erweitern, beispielsweise um einen Pseudonymgenerator, der auf Grundlage eines komplexen Algorithmus arbeitet, oder um einen Generator, welcher eine fortlaufende Nummer generiert. 


\section{Prototypische Implementierung}

Im Rahmen der prototypischen Implementierung wurde ein Probandenmanagementsystem, basierend auf dem Architekturkonzept, entwickelt.

\subsection{Umsetzung des Architekturkonzepts}

Die Struktur des Probandenmanagementsystems umfasst zehn Komponenten (siehe Abbildung 28). Dabei bildet die übergeordnete Komponente pm (=Probandenmanagement) das Rahmenkonstrukt des gesamten Systems und besteht aus den drei untergeordneten Komponenten pmparent, pm-application und pm-assembly. Diese drei Komponenten sowie deren untergeordnete Komponenten wurden in Form von Maven-Projekten realisiert.

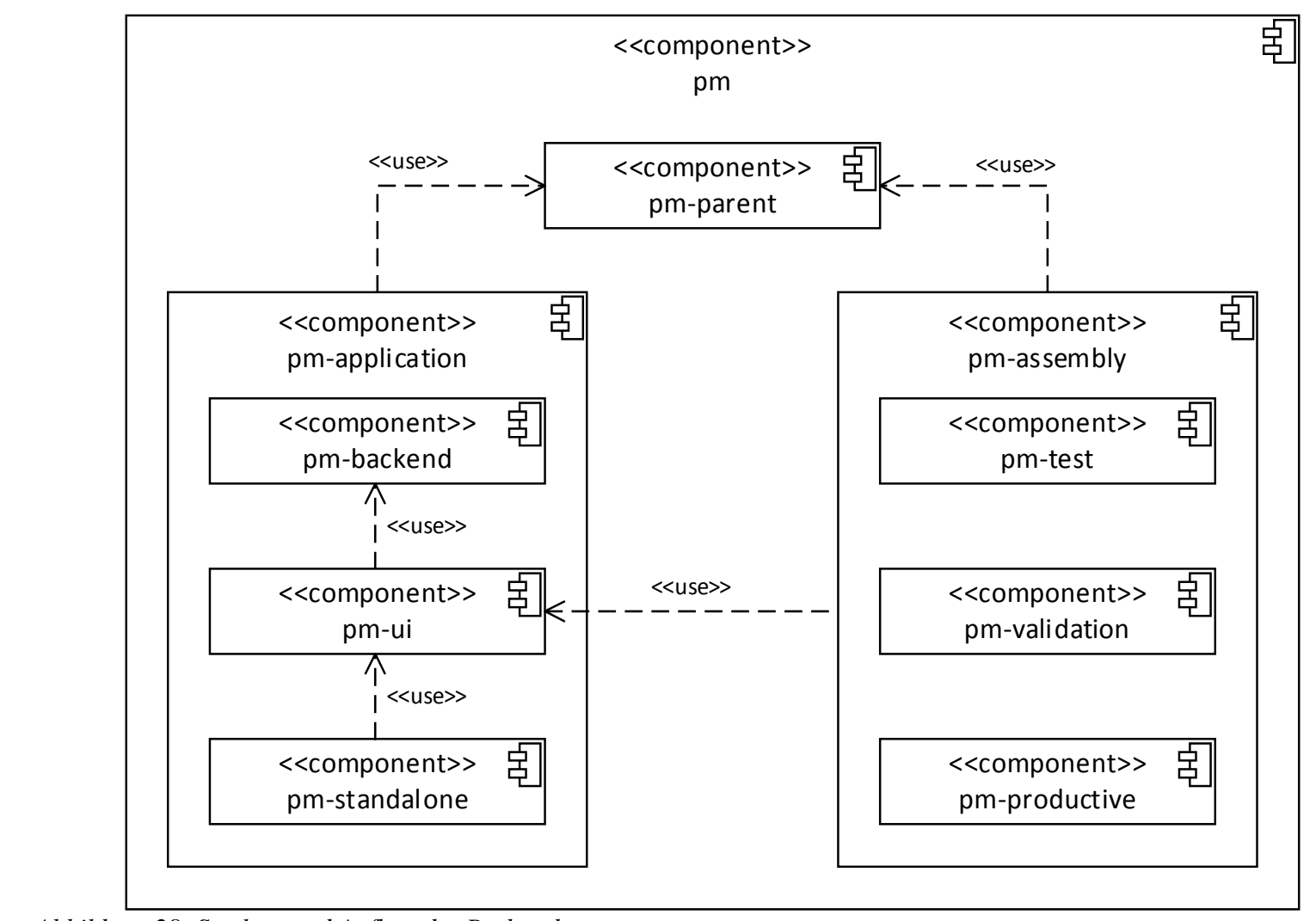

Abbildung 28. Struktur und Aufbau des Probandenmanagementsystems

Innerhalb der Komponente pm-parent werden alle benötigten Abhängigkeiten zu externen Softwarebibliotheken mittels Maven konfiguriert. Alle Komponenten des Prototyps verweisen direkt oder indirekt immer auf pm-parent.

Die Komponente pm-application besteht aus drei untergeordneten Komponenten und umfasst die Applikationslogik des Probandenmanagementsystems. Die Applikationslogik wurde vollständig in der Programmiersprache Java entwickelt. Den Kern des Systems bildet die Komponente pm-backend. Sie beinhaltet die Umsetzung des entwickelten Architekturkonzepts. Die Komponente pm-backend umfasst die vollständige Implementierung der UML-Klassen in Java sowie die Abbildung des Domänenmodells in einer relationalen Datenbank mit Hilfe von Hibernate. Hibernate ermöglicht, alle Änderungen an den Datensätzen nachzuvollziehen (A-R-4). 
Die nachfolgende Abbildung 29 zeigt die Änderungsnachverfolgung in Hibernate auf Datenbankebene.

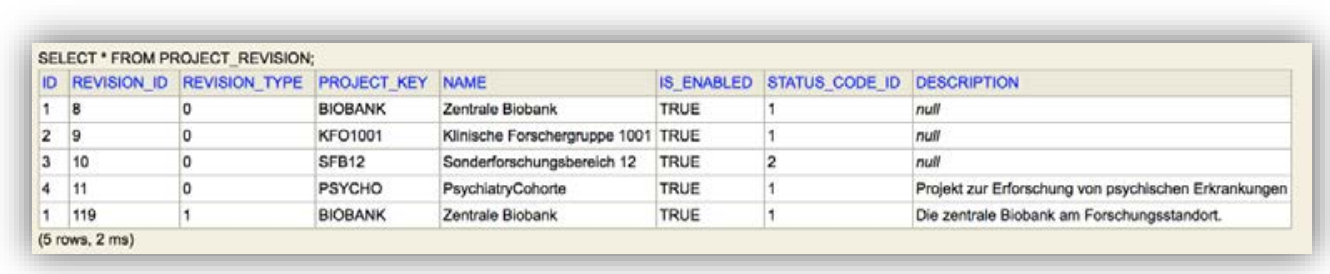

Abbildung 29. Änderungsnachverfolgung auf Datenbankebene

In der ersten Zeile (REVISION_ID = 8) wurde das Projekt anlegt. Im späteren Verlauf wurde die Beschreibung des Projekts ergänzt (REVISION_ID = 119).

Die Komponente pm-backend wird von der Komponente pm-ui genutzt, in welcher die grafische Benutzeroberfläche des Systems enthalten ist. Die letzte untergeordnete Komponente ist pm-standalone. Diese beinhaltet alle notwendigen Softwarebibliotheken, um das Probandenmanagementsystem lokal auf einem Computer zu Testzwecken bzw. zur Entwicklung zu betreiben.

Da der produktive Betrieb des Probandenmanagementsystems auf einem Applikationsserver erfolgt, umfasst die Komponente pm-assembly die dafür notwendige Konfiguration. Unterteilt wird diese Komponente in eine Test-, Validierungs- und Produktionsumgebung: pm-test, pmvalidation und pm-productive. Dies ist notwendig, da abhängig von der jeweiligen Umgebung unterschiedliche Konfigurationen notwendig sind, z. B. welche Implementierung des RecordLinkageAdapter genutzt werden soll. Innerhalb der drei untergeordneten Komponenten werden die zu verwendenden Implementierungen bzw. Adapter der sekundären Schnittstellen festgelegt. Dies erfolgt mit Hilfe der Softwarebibliothek Spring. Durch die Implementierung von Adaptern kann so die Integration in die IT-Infrastruktur der einzelnen Forschungsprojekte (AR-7) und der Universitätsmedizin Göttingen (A-R-8) sichergestellt werden.

\subsection{Benutzeroberfläche}

Die Benutzeroberfläche des Probandenmanagementsystems wurde in Form einer Webanwendung umgesetzt (A-R-1). Durch die Verwendung der Softwarebibliothek Vaadin zur Erstellung der Benutzeroberfläche ist diese in allen gängigen Webbrowsern lauffähig [159]. Darüber hinaus wird keine zusätzliche Software, wie beispielsweise Flash, benötigt (A-R-2) und die Oberfläche kann in verschiedenen Sprachen dargestellt werden (A-R-3) [141].

Zur Sicherung des Probandenmanagementsystems besteht die Benutzeroberfläche aus zwei Teilen: Loginseite (siehe Abbildung 30) und Anwendungsseite (siehe Abbildung 31 am Beispiel der Probandenübersicht). 
Die Loginseite des Probandenmanagementsystems ermöglicht die Anmeldung der Benutzer zum System. Dies kann entweder über die zentrale Benutzerauthentifizierung der Universitätsmedizin Göttingen erfolgen oder über eine interne Benutzerverwaltung des Probandenmanagementsystems.

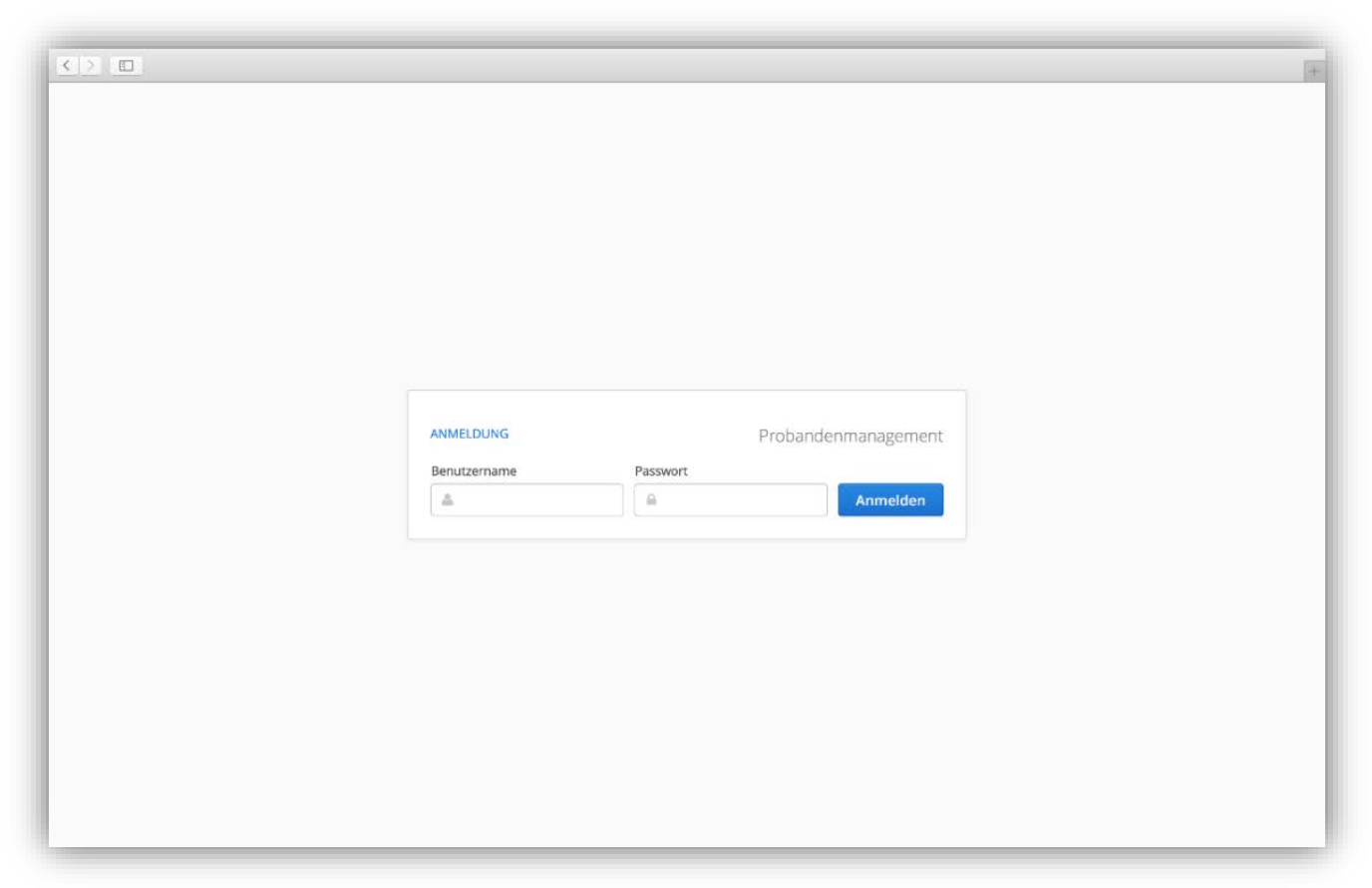

Abbildung 30. Loginseite des Probandenmanagementsystems

Nach erfolgreicher Anmeldung am System öffnet sich für den Benutzer die eigentliche Anwendungsseite. Diese ist unterteilt in einen Menü- und Arbeitsbereich (siehe Abbildung 31 am Beispiel der Probandenübersicht).

Der Menübereich besteht aus den Menükategorien Dashboard, Projektübersicht und Administration. Nachfolgend werden diese Menükategorien einzeln erläutert.

\subsubsection{Dashboard}

Die Menükategorie Dashboard bietet den Benutzern unter dem Menüpunkt Probandenübersicht eine vollständige Liste Ihrer Probanden (siehe Abbildung 31). Diese Übersicht basiert auf der Zuordnung von Benutzern zu Forschungsprojekten, die wiederum über die Forschungsakte Probanden zugeordnet sind.

In der Probandenübersicht werden nur Forschungsprojekte angezeigt, die einem Benutzer zugeordnet sind. So ist beispielsweise die Probandin „Nora Singer“ noch einem weiteren Forschungsprojekt zugeordnet. Dies kann der Benutzer jedoch nicht sehen, da er keine entsprechenden Rechte für das Projekt besitzt (vgl. Abbildung 47). Neben einer Suchfunktion bietet die Probandenübersicht dem Benutzer eine Detailansicht für jeden Probanden (siehe Abbildung 32). 


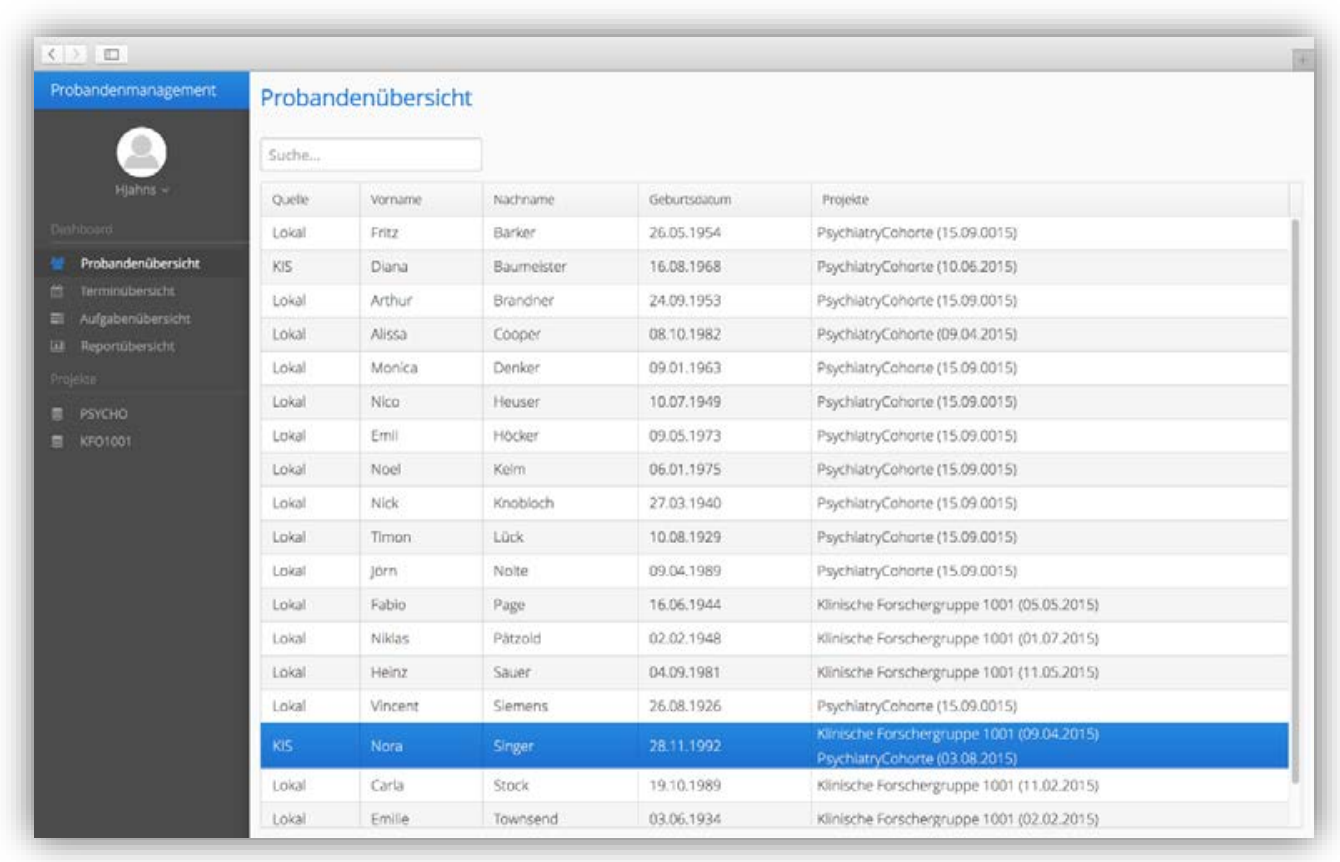

Abbildung 31. Probandenübersicht des Probandenmanagementsystems

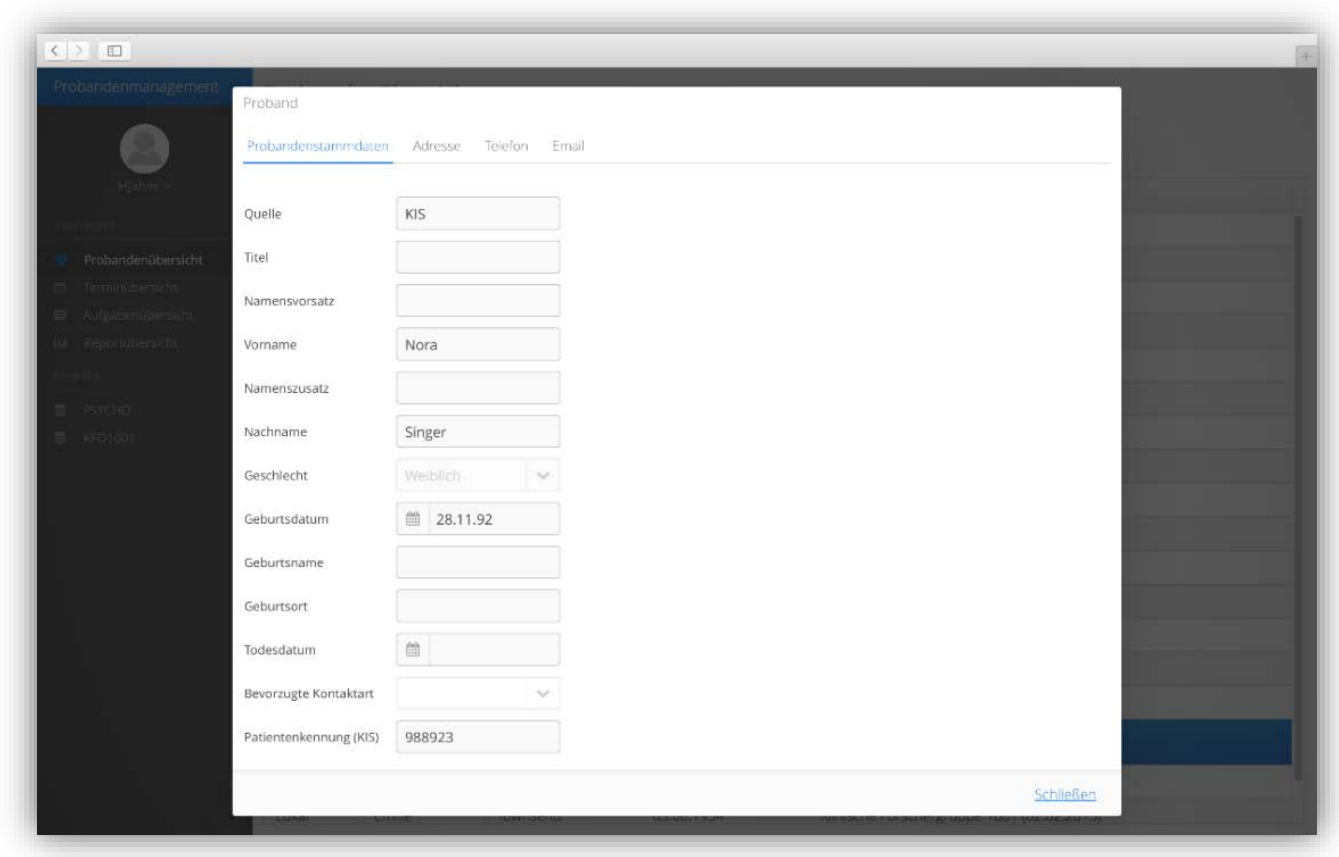

Abbildung 32. Detailansicht eines Probanden im Probandenmanagementsystem 


\subsubsection{Projektübersicht}

In der Menükategorie Projektübersicht befindet sich eine Liste aller Forschungsprojekte, welche dem angemeldeten Benutzer zugeordnet sind (siehe Abbildung 33 im linken Bereich).

Wählt der Benutzer ein Forschungsprojekt aus, so gelangt er auf die Übersichtsseite des ausgewählten Forschungsprojekts. Die projektspezifische Übersichtsseite umfasst die Metadaten, welche zu dem Projekt erfasst wurden. Neben der Übersichtsseite umfasst die Projektansicht noch die Forschungsakten des ausgewählten Forschungsprojekts (siehe Abbildung 33).

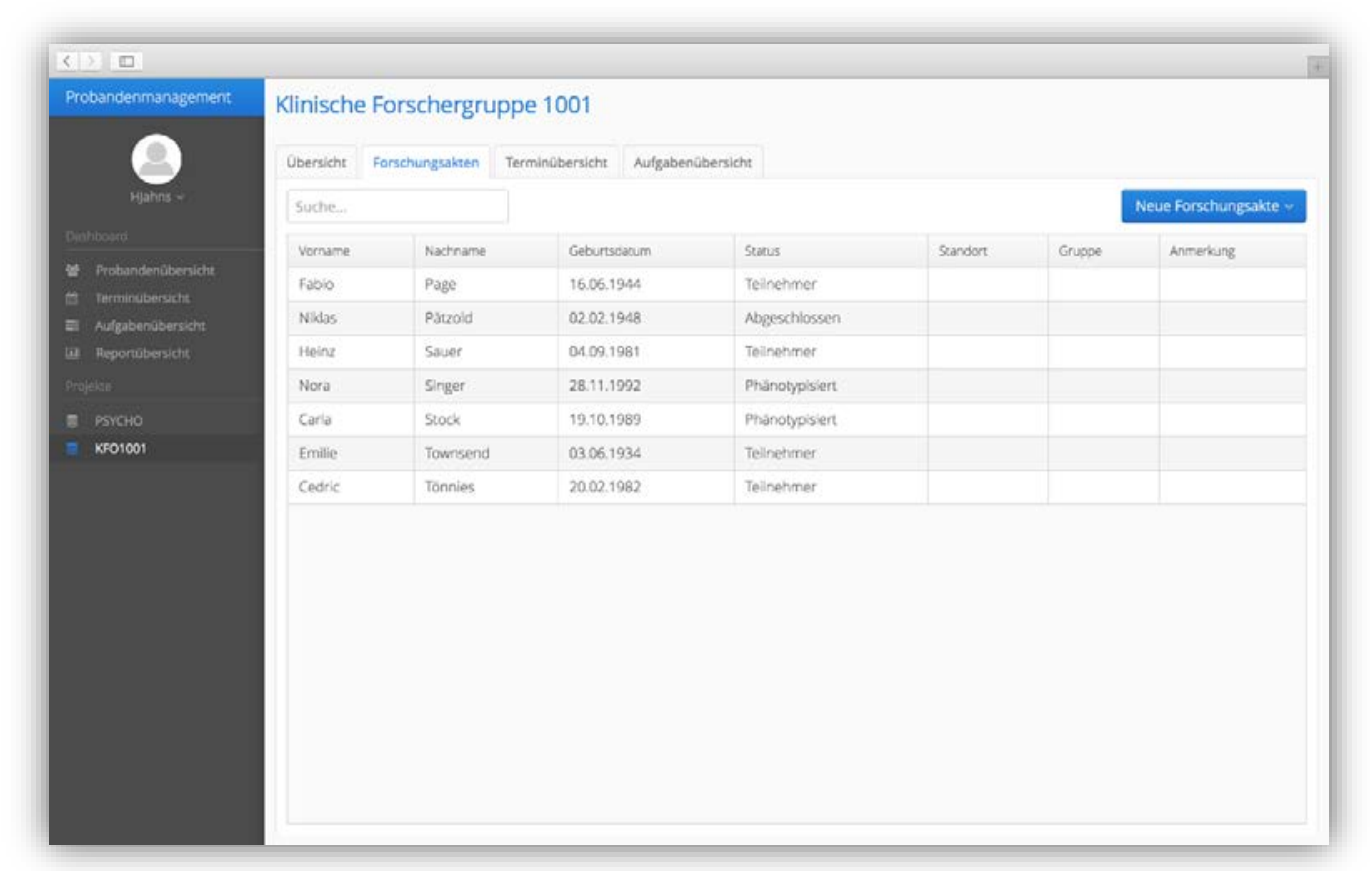

Abbildung 33. Forschungsaktenansicht eines Projekts im Probandenmanagementsystem

Die Forschungsaktenübersicht bietet dem Benutzer eine Übersicht über alle Probanden des Forschungsprojekts. Dies ist vergleichbar mit der Probandenübersicht. Neben dem Namen und Geburtsdatum des Probanden umfasst die Übersicht beispielsweise noch den Rekrutierungsstatus. Darüber hinaus verfügt das Probandenmanagementsystem noch über eine Detailansicht der Forschungsakte (siehe Abbildung 34). Hierzu wählt der Benutzer die betreffende Forschungsakte aus und erhält nachfolgend die Detailansicht. Die Detailansicht der Forschungsakte umfasst alle Datensätze, welche zu dem Probanden im Rahmen des Forschungsprojektes im Probandenmanagementsystem erfasst wurden. Zusätzlich zur Übersicht umfasst die Forschungsakte die Stammdaten des Probanden sowie den Bereich Pseudonymisierung. 


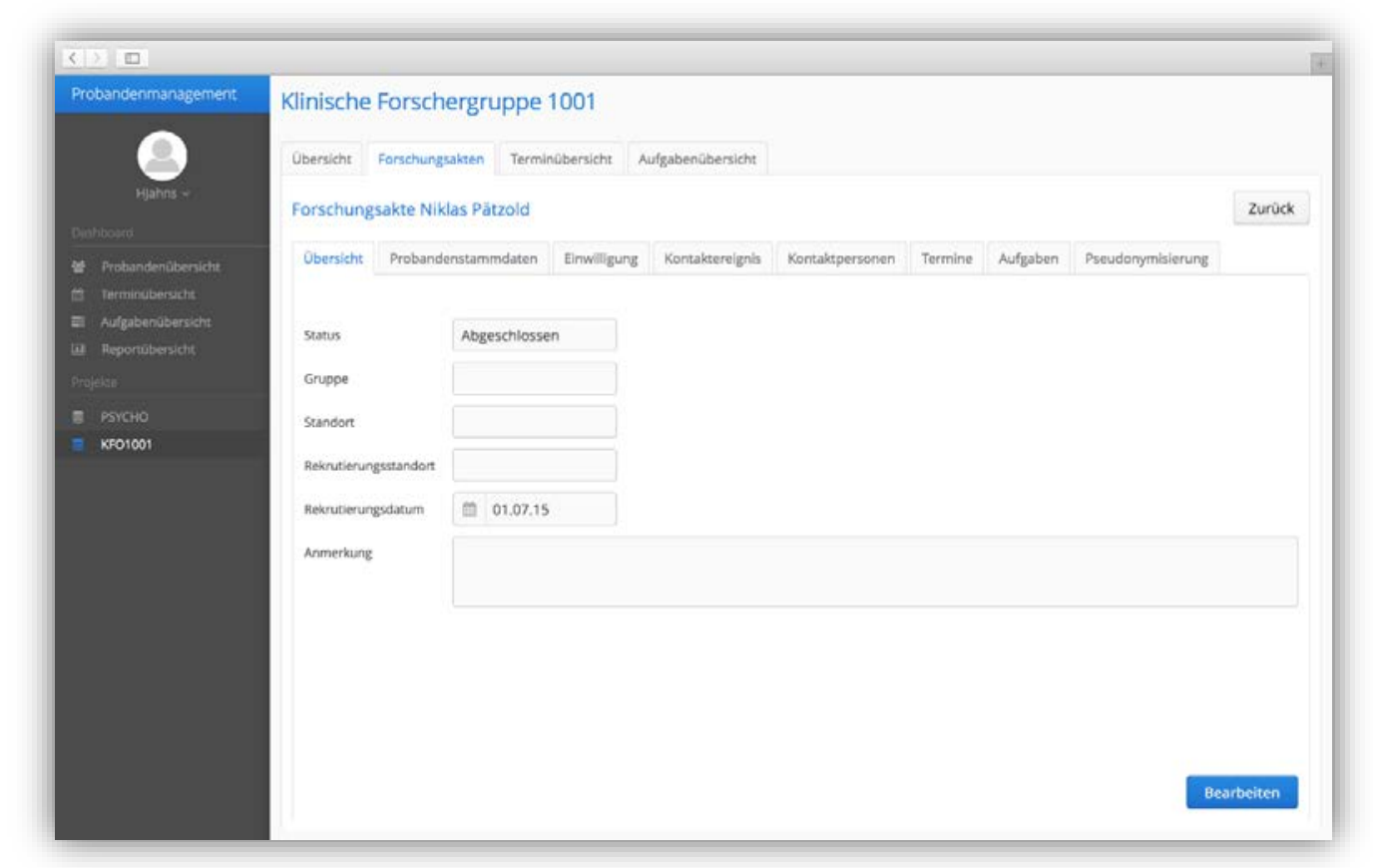

Abbildung 34. Forschungsakte eines Probanden im Probandenmanagementsystem

\subsubsection{Administration}

Die letzte Menükategorie Administration ist nur für Administratoren des Systems zugänglich. Sie umfasst die Bereiche Projektverwaltung, Benutzerverwaltung sowie Systemeinstellungen.

Primäre Aufgabe der Projektverwaltung ist die Konfiguration der Forschungsprojekte, welche im Probandenmanagementsystem verwaltet werden. Neben einer administrativen Projektübersicht (vgl. Abschnitt 6.2) bietet das System eine Detailansicht des jeweiligen Forschungsprojekts (siehe Abbildung 35).

In der Detailansicht werden alle Konfigurationen von einem Administrator für ein Forschungsprojekt vorgenommen. Dies umfasst die Angabe von Metadaten zum Projekt, Erstellung von Codes, Hinterlegung von Forschungssystemen, Pseudonymgeneratoren sowie externen Systemen zur Pseudonymisierung und die Zuordnung von Rechten zu Rollen.

Die Zuordnung von Benutzern zu Forschungsprojekten erfolgt in der Benutzerverwaltung. Innerhalb dieser können Administratoren Benutzer anlegen und diesen Rollen zuordnen (siehe Abbildung 36). Ein Benutzer kann ein lokaler Benutzer sein oder aus der zentralen Benutzerverwaltung der Universitätsmedizin Göttingen stammen.

Die letzten Einträge der Menükategorie bilden die Systemkonfiguration und die Reportverwaltung. In diesen Bereichen können Einstellungen für das gesamte System vorgenommen bzw. Reports erstellt werden. 


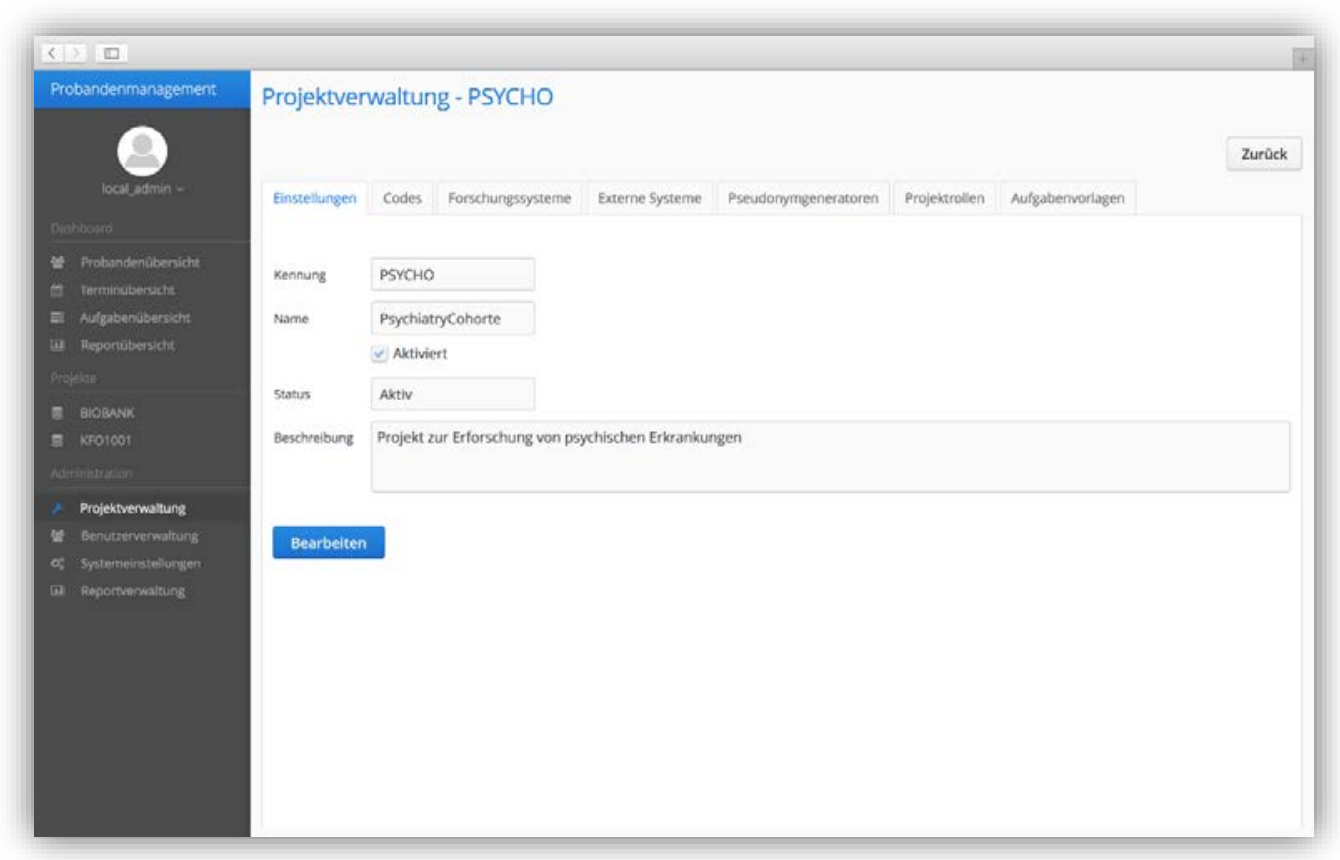

Abbildung 35. Administrative Detailansicht eines Forschungsprojekts im Probandenmanagementsystem

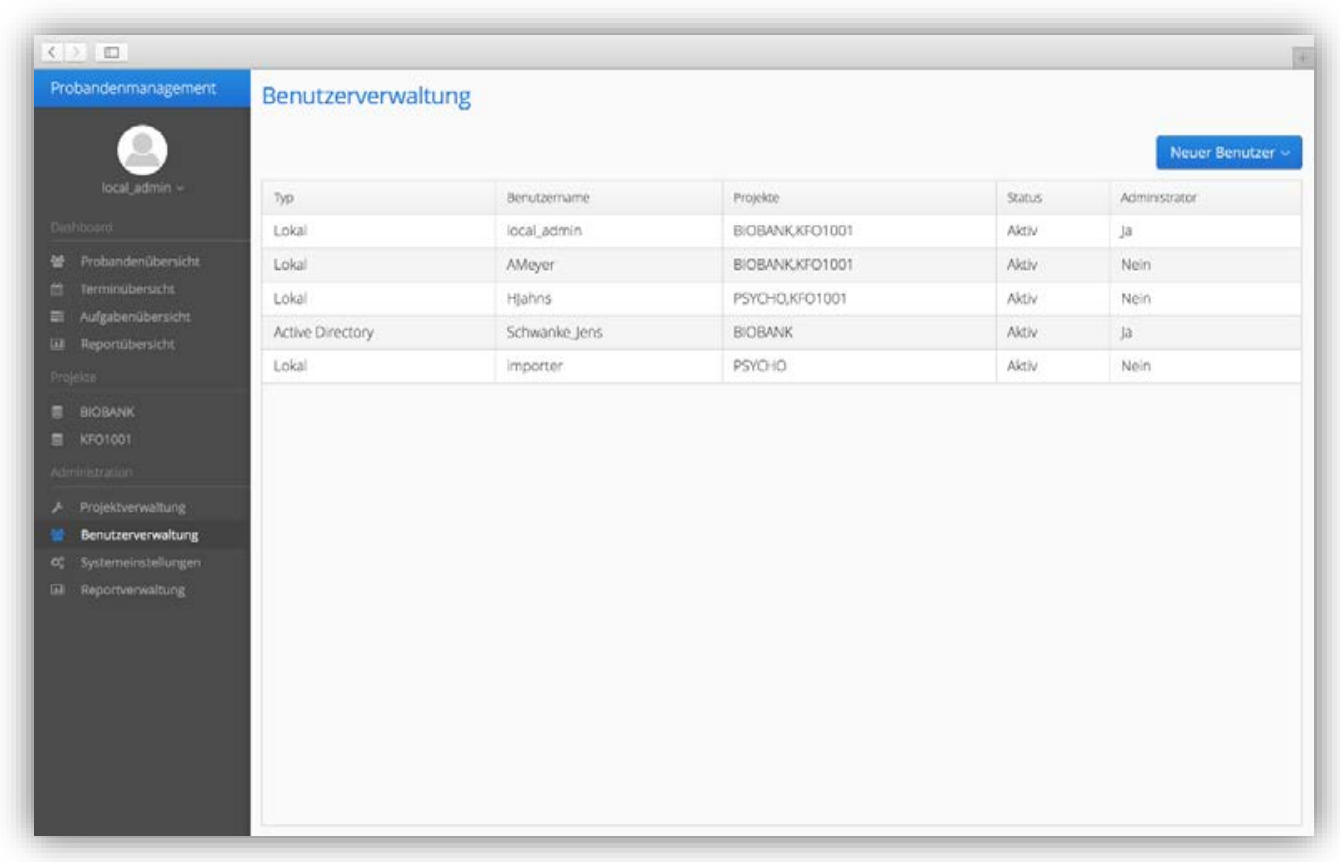

Abbildung 36. Benutzerverwaltung im Probandenmanagementsystem 


\section{Funktionsprüfung}

Die Funktionsprüfung ist Bestandteil der Validierung des entwickelten Probandenmanagementsystems und soll untersuchen, welche funktionalen Anforderungen durch das System abgebildet werden. Zur Strukturierung der Funktionsprüfung wird die in Abschnitt 3.3.1 getroffene Kategorisierung der funktionalen Anforderungen verwendet: Authentifizierung und Autorisierung (Abschnitt 6.1), Verwaltung von Forschungsprojekten (Abschnitt 6.2), Verwaltung von Probanden (Abschnitt 6.3), Verwaltung von Einwilligungserklärungen (Abschnitt 6.5) und Pseudonymisierung (Abschnitt 6.5).

Zum Abschluss dieses Kapitels werden in Abschnitt 6.6 die Ergebnisse der Funktionsprüfung im Hinblick auf die Erfüllung der identifizierten Ziele und Szenarien zusammengefasst.

\subsection{Authentifizierung und Autorisierung}

Die Sicherung des Probandenmanagementsystems gegen unautorisierte Zugriffe erfolgt zum einen durch eine Authentifizierung der Benutzer mittels Benutzername und Passwort (siehe Abbildung 37) und zum anderen durch das Rechte- und Rollenmanagement.

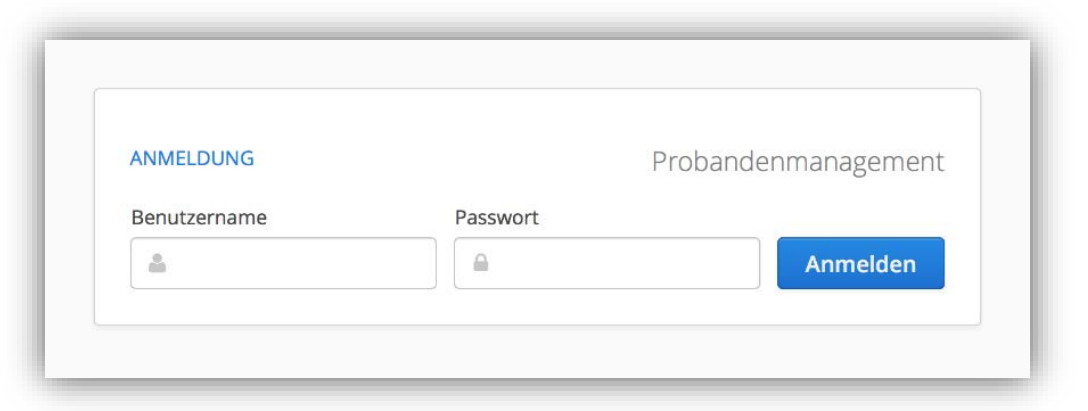

Abbildung 37. Login des Probandenmanagementsystems zur Benutzerauthentifizierung

Dabei kann die Authentifizierung eines Benutzers entweder über einen zentralen Verzeichnisdienst in Form eines Active Directory oder durch die systeminterne Benutzerverwaltung erfolgen. Die Konfiguration des zentralen Verzeichnisdiensts wird über die Systemkonfiguration vorgenommen. Hierdurch wird die Authentifizierung eines Benutzers über die zentrale Benutzerverwaltung der Universitätsmedizin Göttingen ermöglicht (A-F-1). Abbildung 38 zeigt die entsprechenden Einstellungen für den Verzeichnisdienst in der Systemkonfiguration.

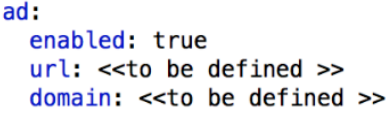

Abbildung 38. Systemkonfiguration zur Einstellung eines zentralen Benutzerverzeichnisdiensts

Darüber hinaus verfügt das Probandenmanagementsystem über eine systeminterne Benutzerverwaltung. Diese kann unabhängig von einem zentralen Verzeichnisdienst genutzt werden. 
Ebenso ist eine parallele Nutzung beider Verfahren möglich. Beim Anlegen eines neuen Benutzers muss ausgewählt werden, ob die Authentifizierung durch ein zentrales System oder über die integrierte lokale Benutzerverwaltung (A-F-2) erfolgt.

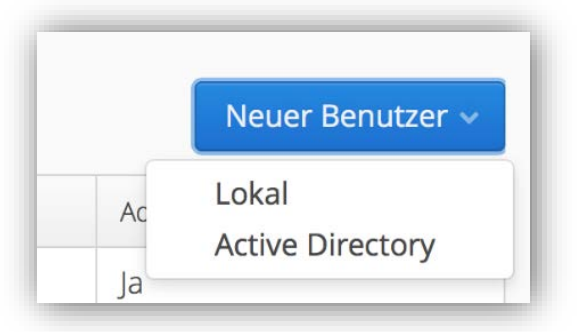

Abbildung 39. Auswahl des Authentifizierungsverfahrens beim Anlegen eines neuen Benutzers

Soll die Authentifizierung lokal erfolgen, muss für den Benutzer zusätzlich ein Passwort angegeben werden. Unabhängig vom Authentifizierungsverfahren ist eine explizite Aktivierung der Benutzer erforderlich. Im gleichen Dialog kann ein Benutzer auch als Administrator definiert werden (siehe Abbildung 40).

Nach erfolgreicher Authentifizierung des Benutzers erfolgt die Autorisierung durch die Rechteund Rollenmanagement (A-F-3). Innerhalb der Benutzerverwaltung kann den Benutzern eine entsprechende Rolle für jedes Forschungsprojekt zugewiesen werden. Jeder Benutzer kann jeweils nur eine Rolle in einem Forschungsprojekt innehaben (siehe Abbildung 40). Die Definition der Rollen ist Gegenstand der Projektverwaltung und wird im nachfolgenden Abschnitt erläutert.

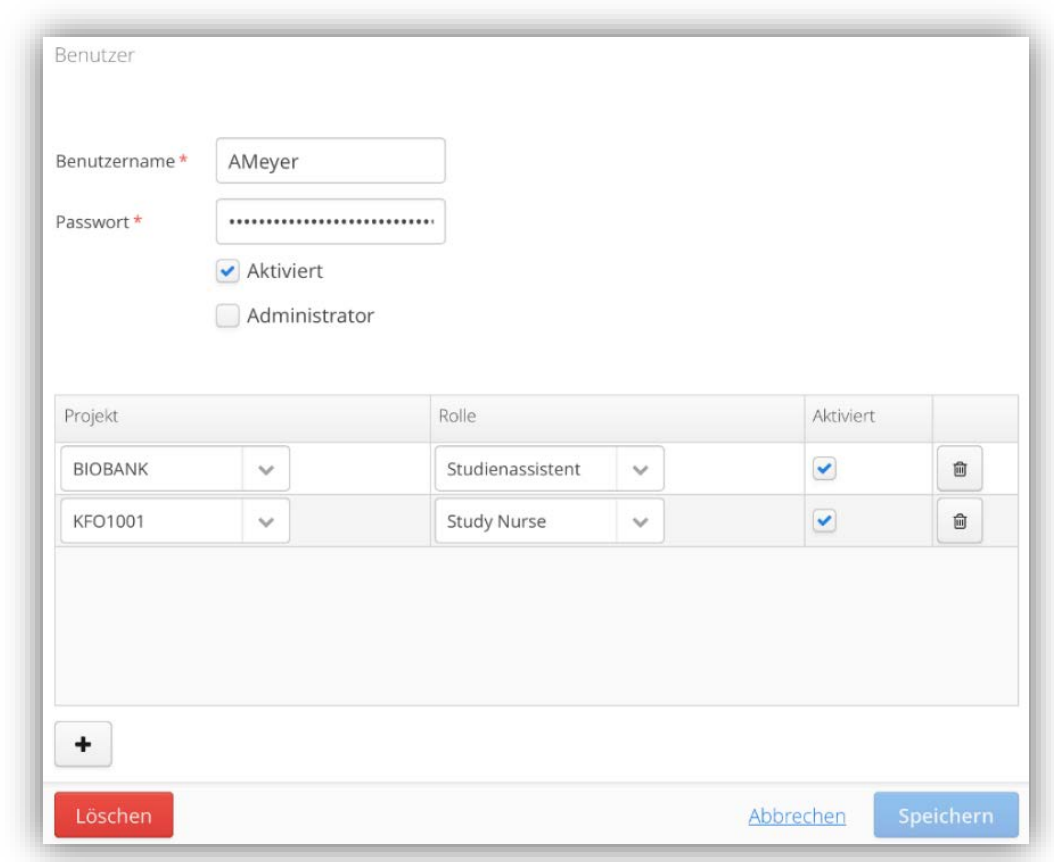

Abbildung 40. Einstellungen für einen lokalen bzw. zentralen Benutzer 


\subsection{Verwaltung von Forschungsprojekten}

Die Verwaltung von Forschungsprojekten erfolgt im Administrationsbereich (siehe Abbildung 41). In diesem können Forschungsprojekte angelegt, bearbeitet und ggf. gelöscht werden.

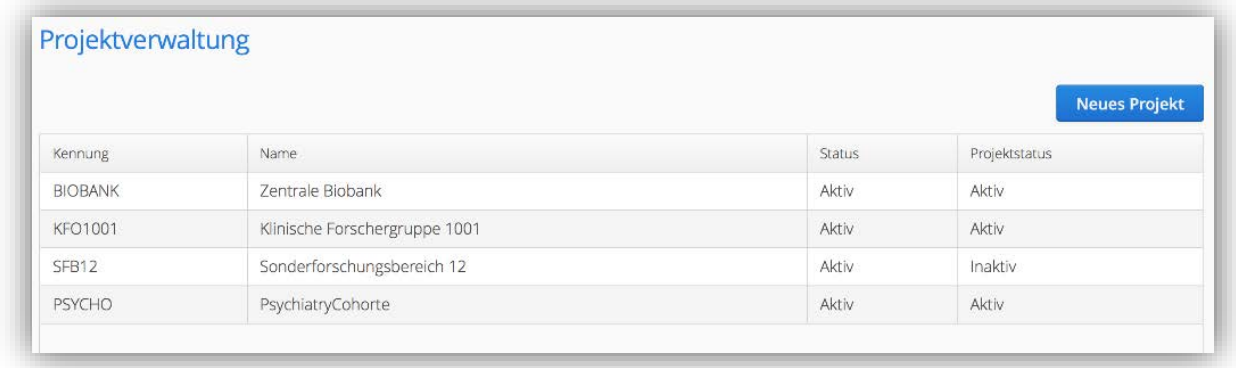

Abbildung 41. Projektverwaltung im Administrationsbereich

Beim Anlegen eines neuen Forschungsprojekts ist die Eingabe einer eindeutigen Kennung, des Projektnamens sowie des Projektstatus notwendig (siehe Abbildung 42). Ebenso kann das Projekt als aktiv oder inaktiv markiert oder um eine Beschreibung optional erweitert werden (A-F4).

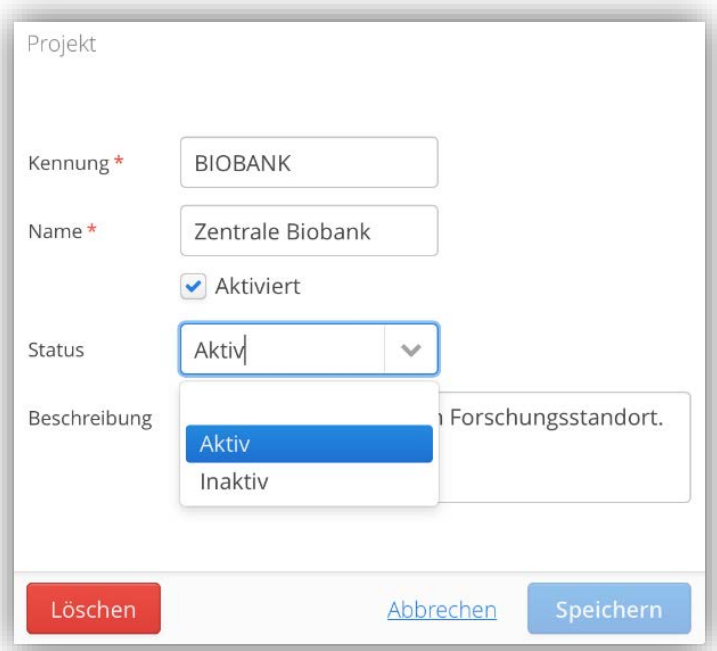

Abbildung 42. Anlegen, Bearbeiten und Löschen von Forschungsprojekten

Nachdem ein Projekt angelegt wurde, können ergänzende Daten über das Forschungsprojekt erfasst werden (siehe Abbildung 43), bspw. die Angabe von zugehörigen Forschungssystemen (A-F-5). 


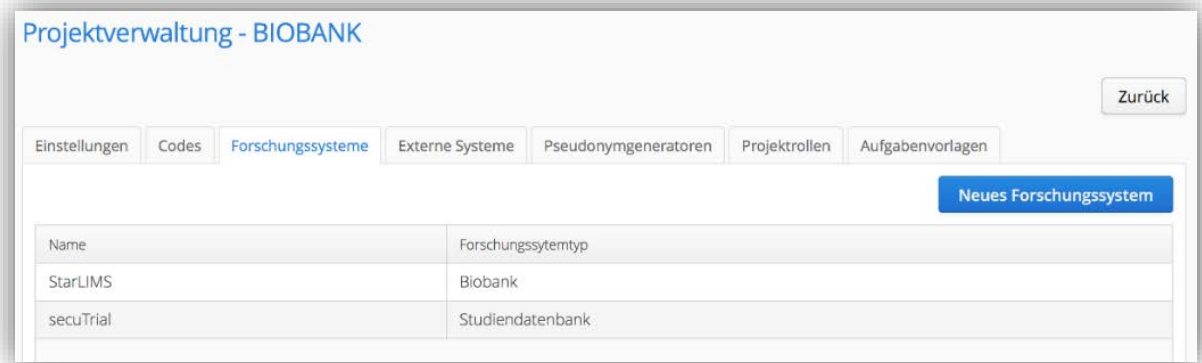

Abbildung 43. Verfügbare Reiter zur Erfassung ergänzender Daten für ein Forschungsprojekt

Innerhalb der Projektverwaltung wird die Zuordnung von Rechten zu projektspezifischen Rollen vorgenommen (A-F-6). Die vom Probandenmanagementsystem vorgegebenen Rechte (siehe Abbildung 44) werden der jeweiligen Rolle zugeordnet. Ein Benutzer kann in einem Forschungsprojekt immer nur eine Rolle wahrnehmen. Jedoch können die Rechte einer Rolle in den Forschungsprojekten unterschiedlich konfiguriert sein (A-F-7).

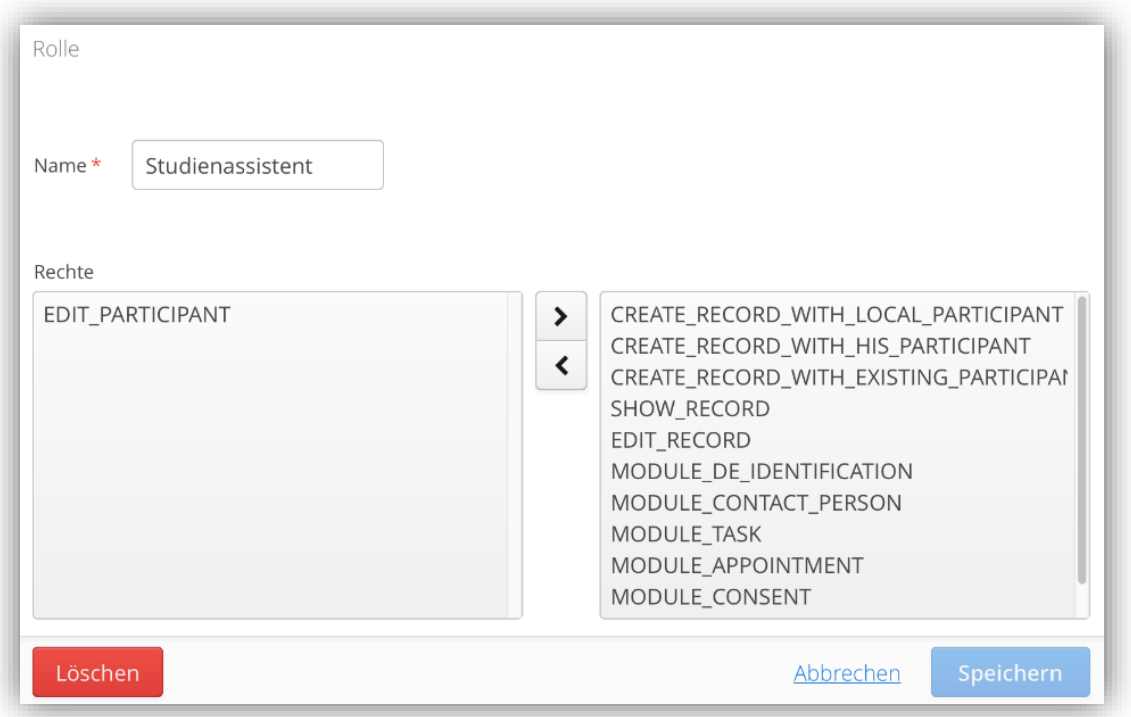

Abbildung 44. Zuordnung von Rechten zu projektspezifischen Rollen

Direkt nach der Anmeldung erhält der Benutzer eine Übersicht über alle ihm zugeordneten Forschungsprojekte. Dies erfolgt, wie in Abbildung 45 dargestellt, über die Menüleiste, welche alle aktiven Forschungsprojekte des Benutzers umfasst (A-F-8). 


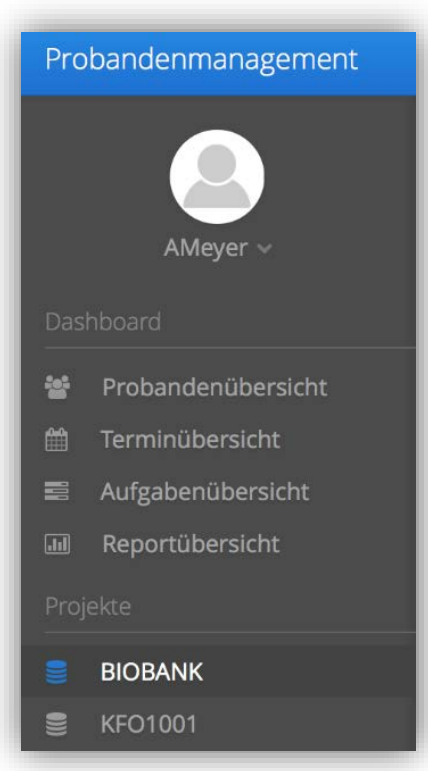

Abbildung 45. Übersicht über die einem Benutzer zugeordneten Forschungsprojekte

\subsection{Verwaltung von Probanden}

Kern des Probandenmanagementsystems bildet die Verwaltung der Probanden. Deren Stammdaten werden zentral im System, wie in Abbildung 46 dargestellt, gepflegt (A-F-9).

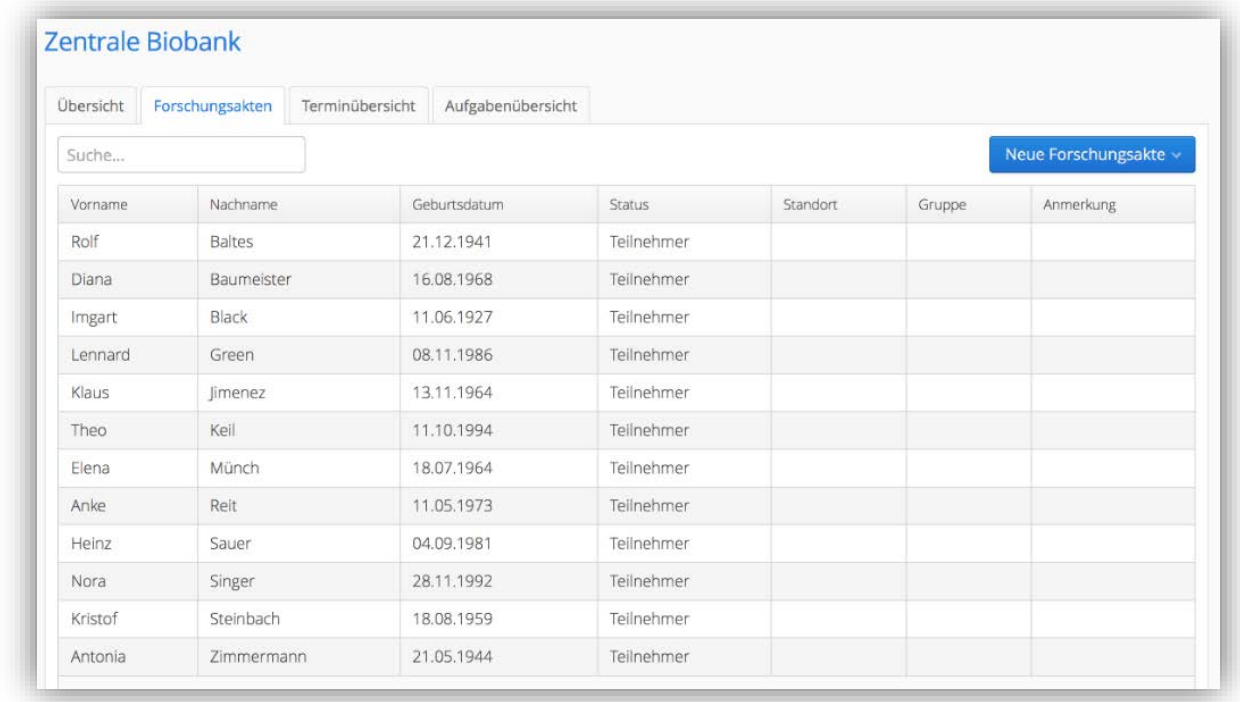

Abbildung 46. Verwaltung der Probandenstammdaten im Forschungsprojekt

Die Zuordnung der Probanden zu Forschungsprojekten erfolgt durch das entwickelte Konzept der Forschungsakte (A-F-10). Eine Forschungsakte verbindet zum einen den Probanden mit dem Forschungsprojekt; zum anderen bildet die Akte den Ausgangspunkt zur Erfassung von projektspezifischen Angaben wie bspw. Pseudonymen (siehe Abbildung 47). 


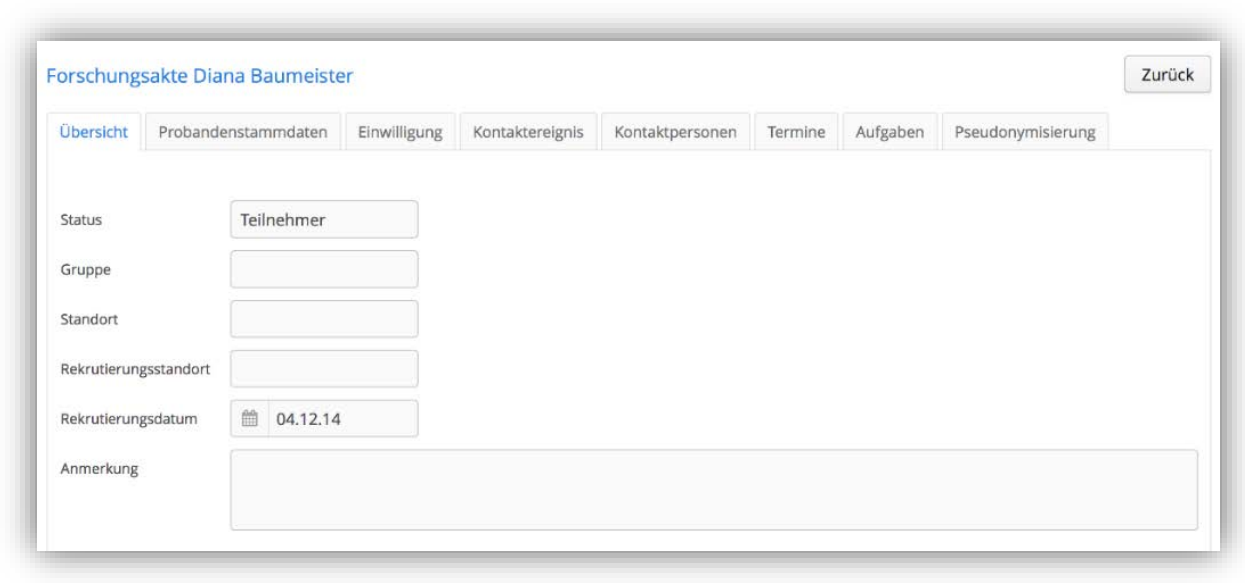

Abbildung 47. Zuordnung von Probanden zu Forschungsprojekten durch die Forschungsakte

Des Weiteren ermöglicht es die Forschungsakte den Benutzern des Probandenmanagementsystems, einen Überblick über alle Probanden zu erhalten. Grundlage für diese Übersicht sind die Zuordnung von Benutzern zu Forschungsprojekten sowie die vorhandene Forschungsakte im jeweiligen Forschungsprojekt (A-F-17). Abbildung 48 zeigt eine Übersicht aller Probanden aus zwei verschiedenen Forschungsprojekten aus Sicht eines angemeldeten Benutzers.

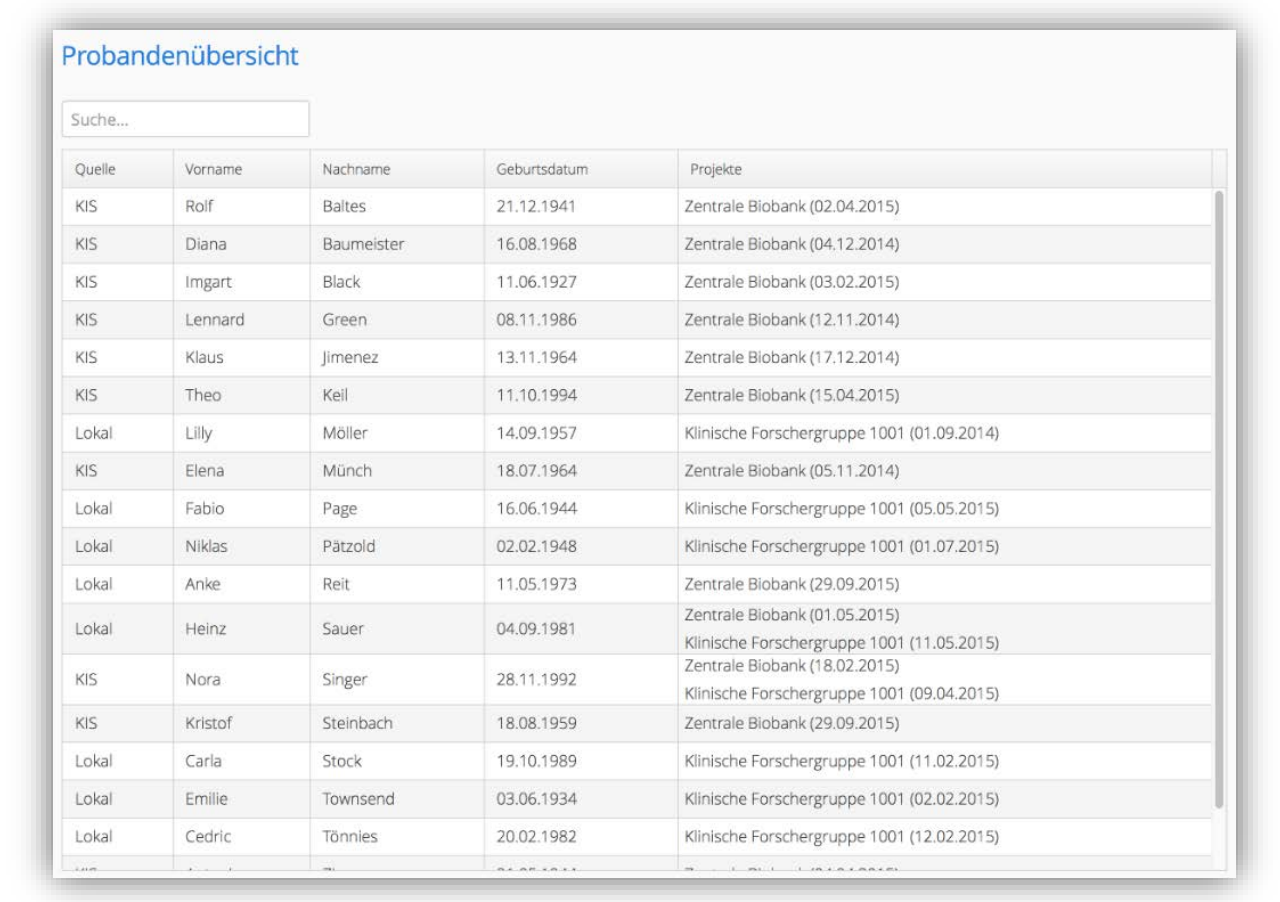

Abbildung 48. Übersicht über alle einem Benutzer zugeordneten Probanden

Aufgrund der projektübergreifenden Verwaltung von Probanden ist die Änderung und Löschung von Probandenstammdaten nur durch Administratoren möglich (A-F-11). Abbildung 49 zeigt die Ansicht auf Probandenstammdaten aus Sicht eines Administrators. Dies soll verdeutlichen, dass nur dem Administrator die Möglichkeit zur Änderung bzw. Löschung von Stammdaten gegeben ist. 


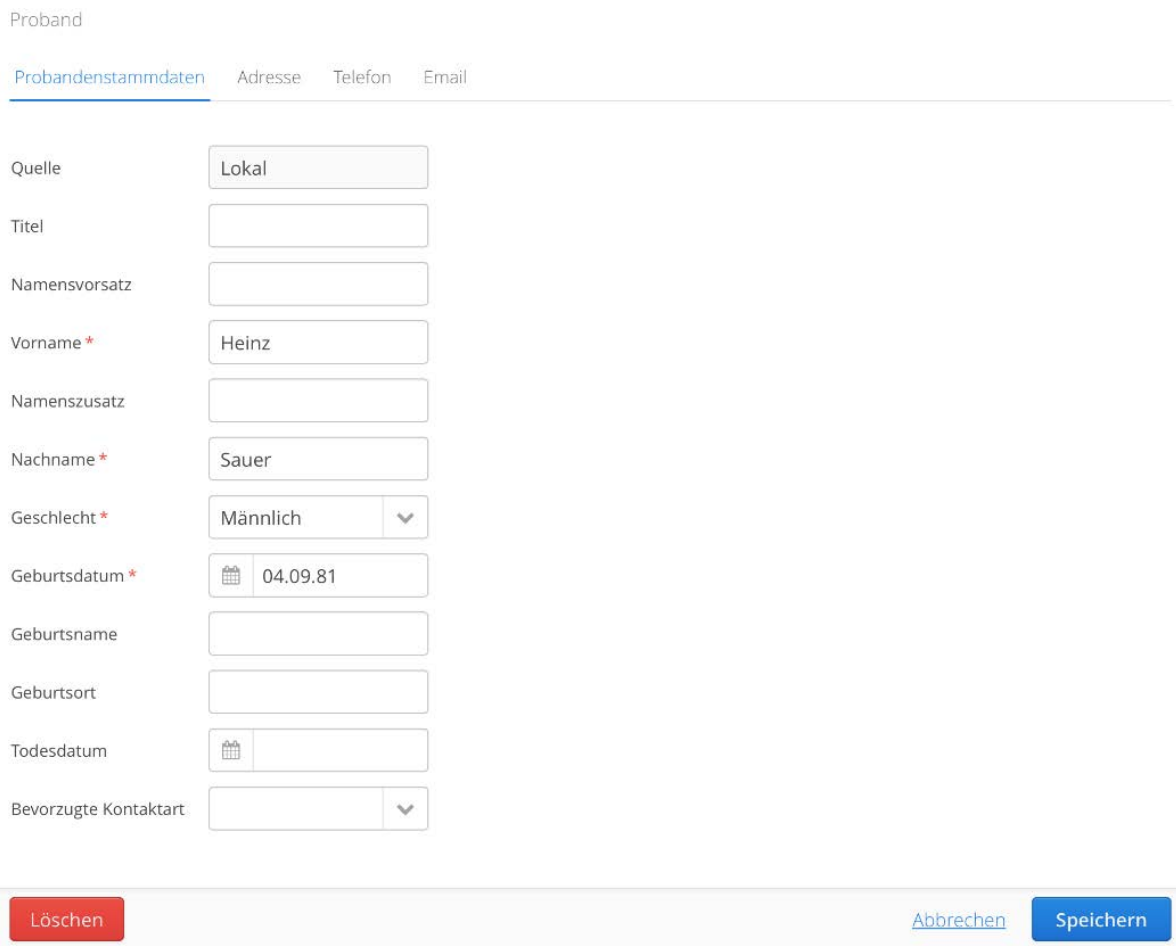

Abbildung 49. Änderung und Löschung von Probandenstammdaten mit entsprechenden Rechten

Die Probandenstammdaten können nur vollständig gelöscht werden, wenn der Proband keinem weiteren Forschungsprojekt mehr zugeordnet ist. Abbildung 50 bis Abbildung 52 zeigen den Löschvorgang eines Probanden, welcher zunächst zwei Forschungsprojekten zugeordnet ist, auf Ebene der Datenbank. Erst wenn auch die Zugehörigkeit zum zweiten Forschungsprojekt entfernt wurde, wird der Datensatz des Probanden vollständig gelöscht (A-F-12).

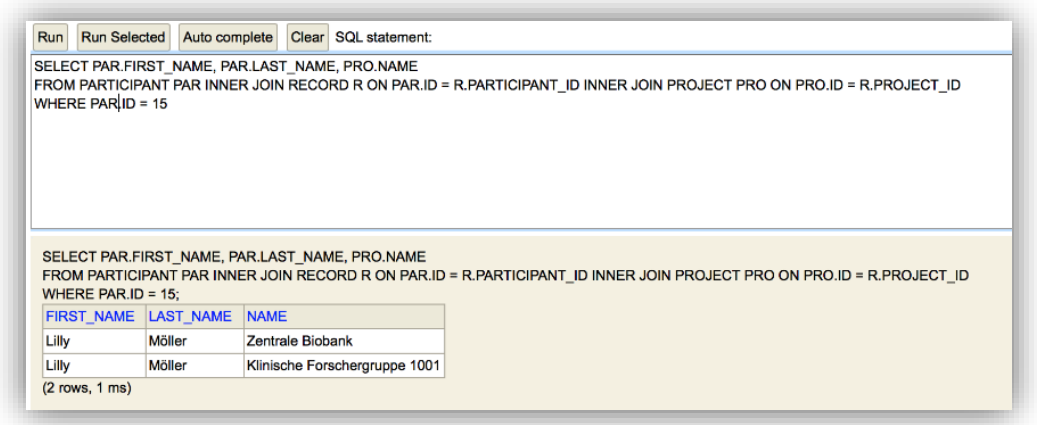

Abbildung 50. Löschen eines Probanden aus Sicht der Datenbank - Schritt 1

Im ersten Schritt weißt der Proband $(X)$ die Zuordnung zu zwei Forschungsprojekten auf. Im nächsten Schritt wird die Zuordnung zum Forschungsprojekt $X$ gelöscht. 


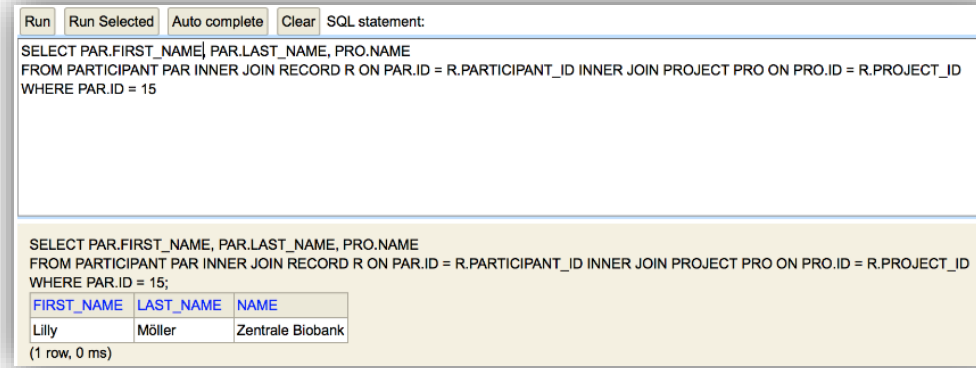

Abbildung 51. Löschen eines Probanden aus Sicht der Datenbank-Schritt 2

Nachdem die Zuordnung des Probanden zum Forschungsprojekt $X$ gelöscht wurde, ist der Proband nur noch dem Forschungsprojekt y zugeordnet. Nachfolgend wird auch diese gelöscht.

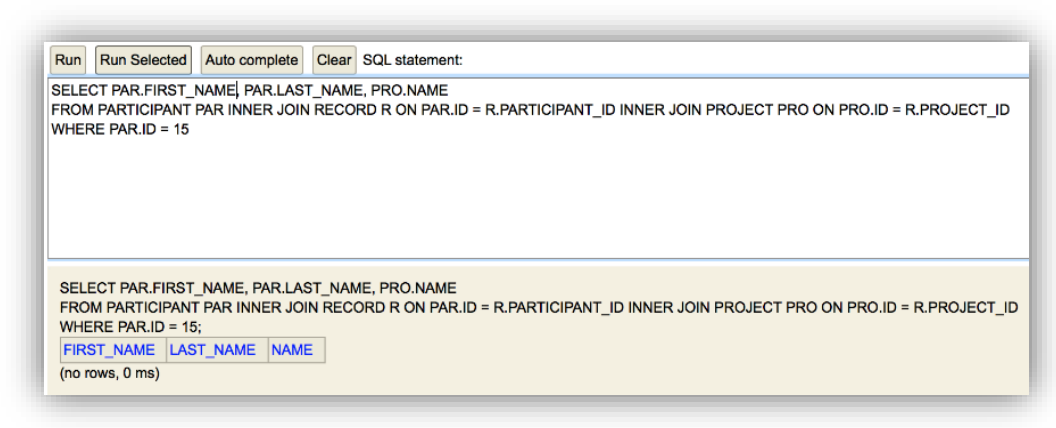

Abbildung 52. Löschen eines Probanden aus Sicht der Datenbank - Schritt 3

Da der Proband keinem Forschungsprojekt mehr zugeordnet ist, wurden seine Daten vollständig gelöscht.

Im Prototyp ist die Bearbeitung und Löschung von Probandenstammdaten den Administratoren vorbehalten. Das Anlegen eines Probanden ist jedoch, abhängig von der jeweiligen Rolle, allen Benutzern möglich. Soll eine neue Forschungsakte angelegt werden, bietet das System den Benutzern drei Möglichkeiten (siehe Abbildung 53 bis Abbildung 56): Anlegen eines neuen Probanden (A-F-15), Auswahl eines Probanden aus einem vorhandenen Forschungsprojekt (A-F14) und den Abruf von Stammdaten aus dem Krankenhausinformationssystem (A-F-13). Auf Grund der Komplexität des Nachrichtenaustauschs mit dem Krankenhausinformationssystem wurde auf die konkrete Implementierung dieser Anforderung verzichtet.

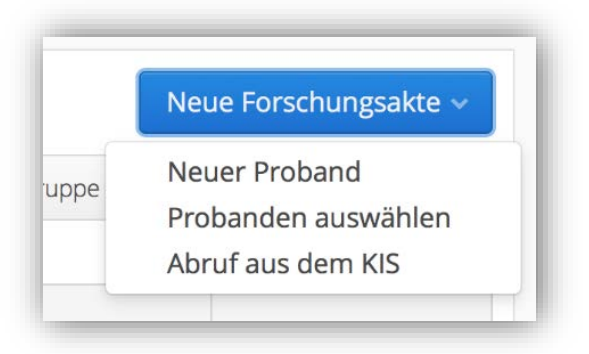

Abbildung 53. Dialog zur Auswahl, wie eine neue Forschungsakte angelegt werden soll 


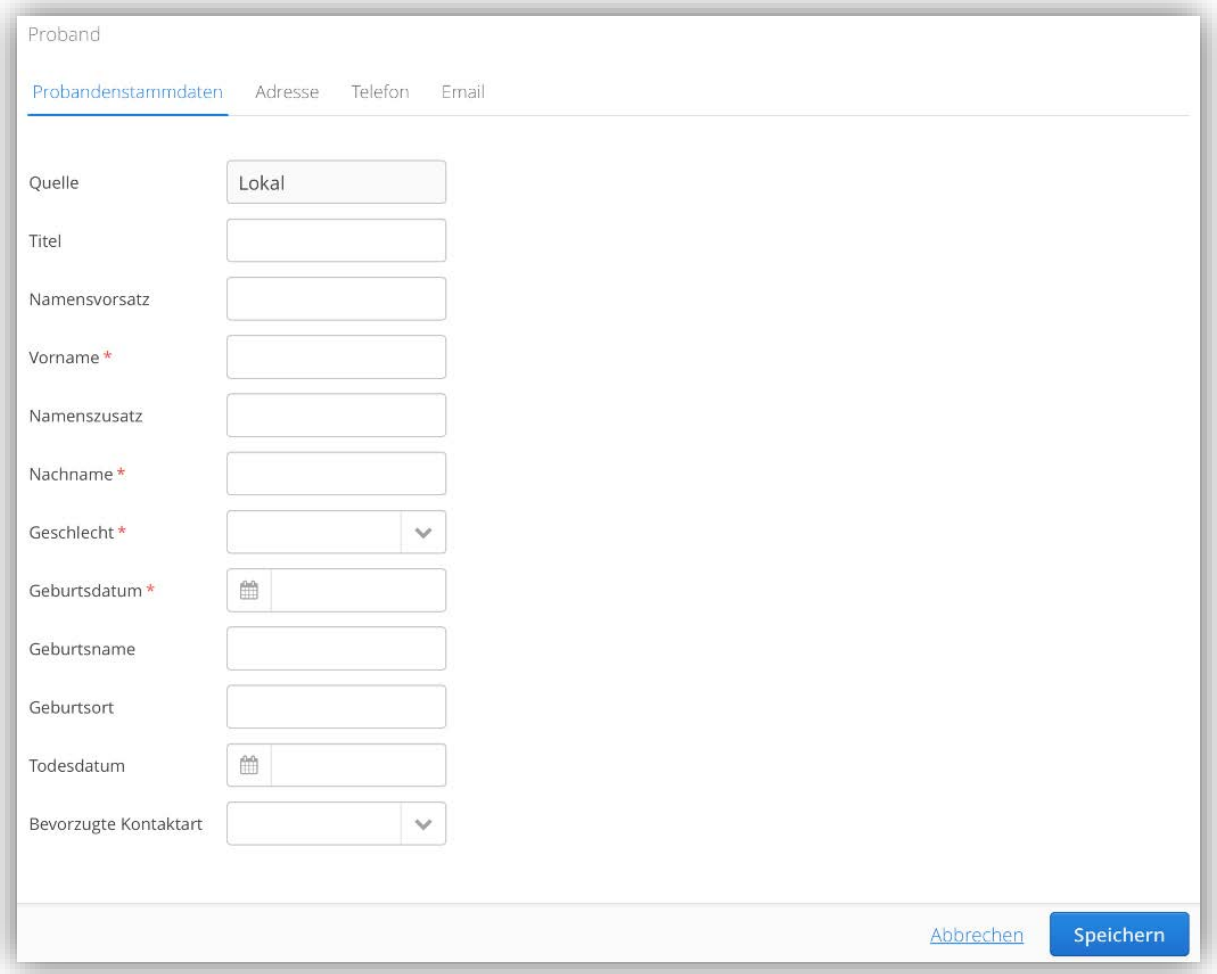

Abbildung 54. Anlegen eines neuen Probanden durch Eingabe der Stammdaten

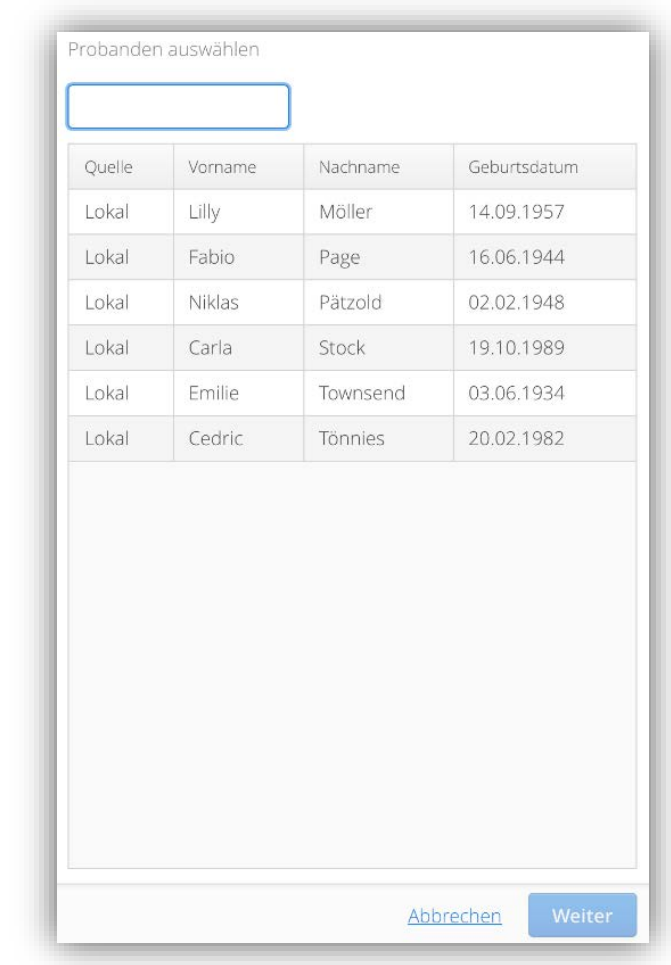

Abbildung 55. Übernahme eines Probanden aus einem vorhandenen Forschungsprojekt 


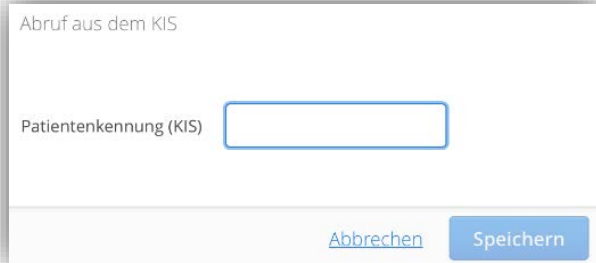

Abbildung 56. Abruf der Probandenstammdaten aus dem KIS

Abschließend werden die Benutzer - wie in Abbildung 57 dargestellt - aufgefordert, zusätzliche Daten für die Forschungsakte des Probanden im Projekt zu dokumentieren, z. B. den Projektstatus (A-F-18).

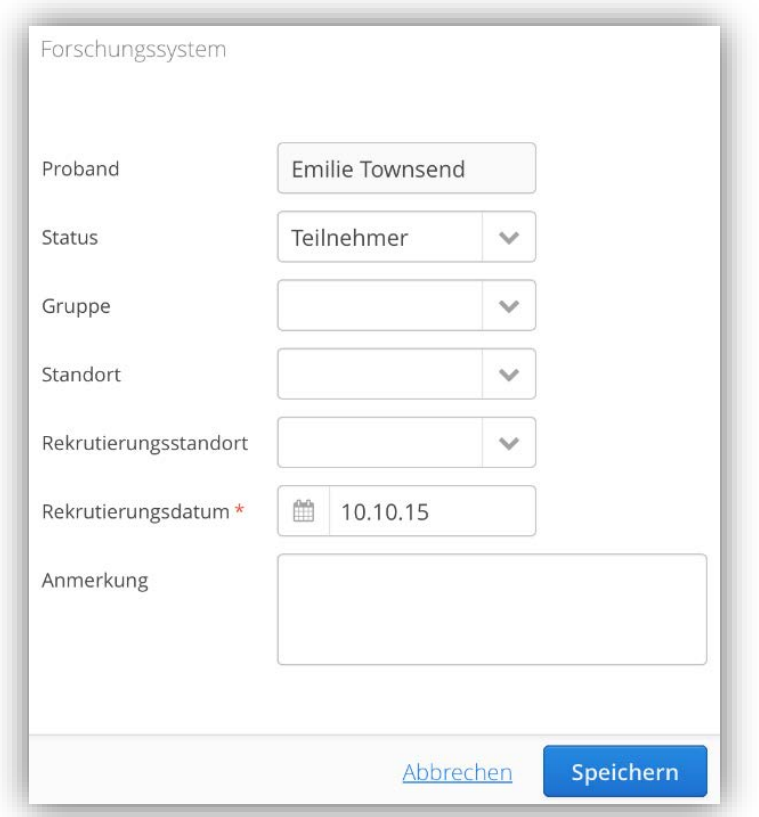

Abbildung 57. Erfassung von projektspezifischen Daten für die Forschungsakte

Die zentrale und projektübergreifende Verwaltung der Probanden erfordert die Erkennung von gleichen Probanden in Form einer Duplikaterkennung (A-F-16). Das nachfolgende Beispiel zeigt in Abbildung 58, wie ein bereits existierender Proband in einem neuen Forschungsprojekt erneut unter Angabe seiner Stammdaten angelegt wird. Die interne Duplikaterkennung stellt in diesem Beispiel fest, dass dieser Proband bereits existiert. In der Probandenübersicht wird der Proband nachfolgend mit zwei Forschungsprojekten aufgeführt (siehe Abbildung 59). 


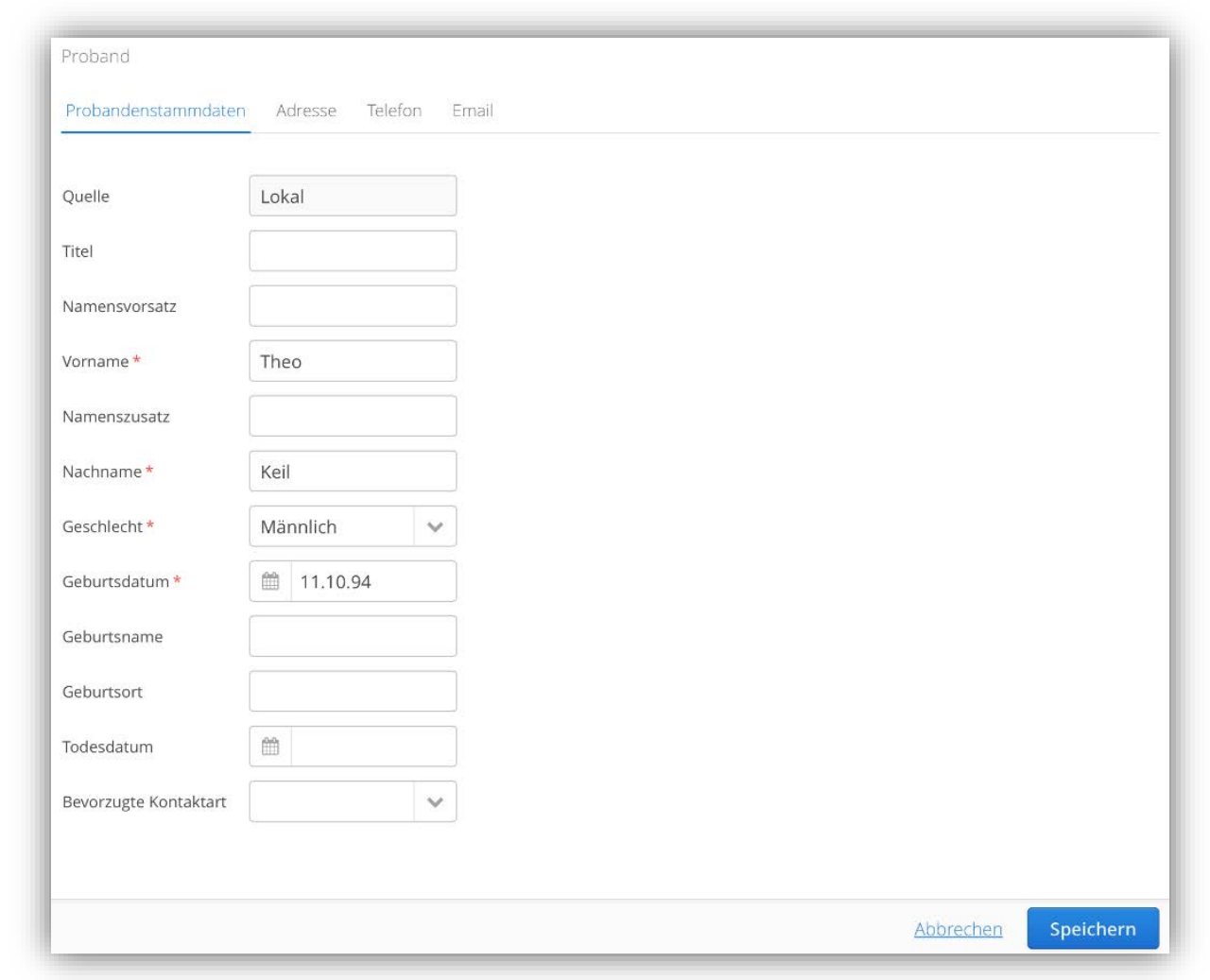

Abbildung 58. Beispiel für die Duplikaterkennung im Probandenmanagementsystem - Schritt 1

Im ersten Schritt wird ein bereits im System geführter Proband (Theo Keil) unter Angabe seiner Stammdaten erneut erfasst.

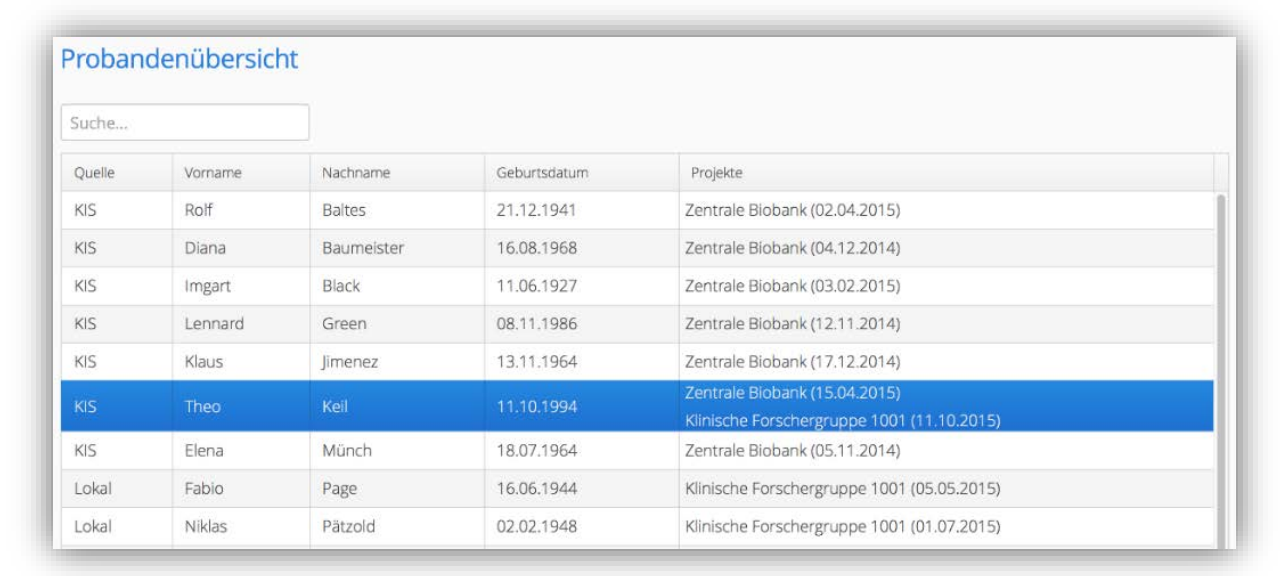

Abbildung 59. Beispiel für die Duplikaterkennung im Probandenmanagementsystem - Schritt 2

Die Duplikaterkennung im Probandenmanagementsystem stellt fest, dass der Proband (Theo Keil) bereits im System erfasst wurde. In der Probandenübersicht wird der Proband nachfolgend mit zwei Forschungsprojekten geführt.

Teilweise bestehen in einigen Forschungsprojekten bereits Listen mit Daten über die teilnehmenden Probanden. Zum Import dieser vorhandenen Probandenstammdaten bietet das Probandenmanagementsystem eine umfangreiche programmierbare Schnittstelle. Zur Validierung dieser Funktion wurde anhand einer Liste mit Beispieldaten (siehe Abbildung 60) ein ImportTool entwickelt (siehe Anhang A.7). Abbildung 61 zeigt den resultierenden Import im Probandenmanagementsystem. 


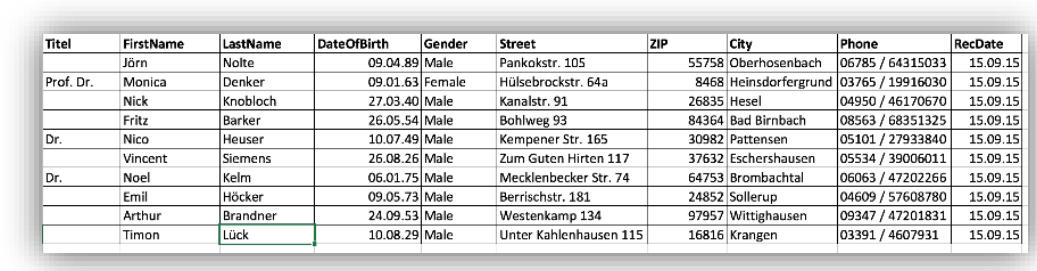

Abbildung 60. Beispieldatensatz für den Import ins Probandenmanagementsystem

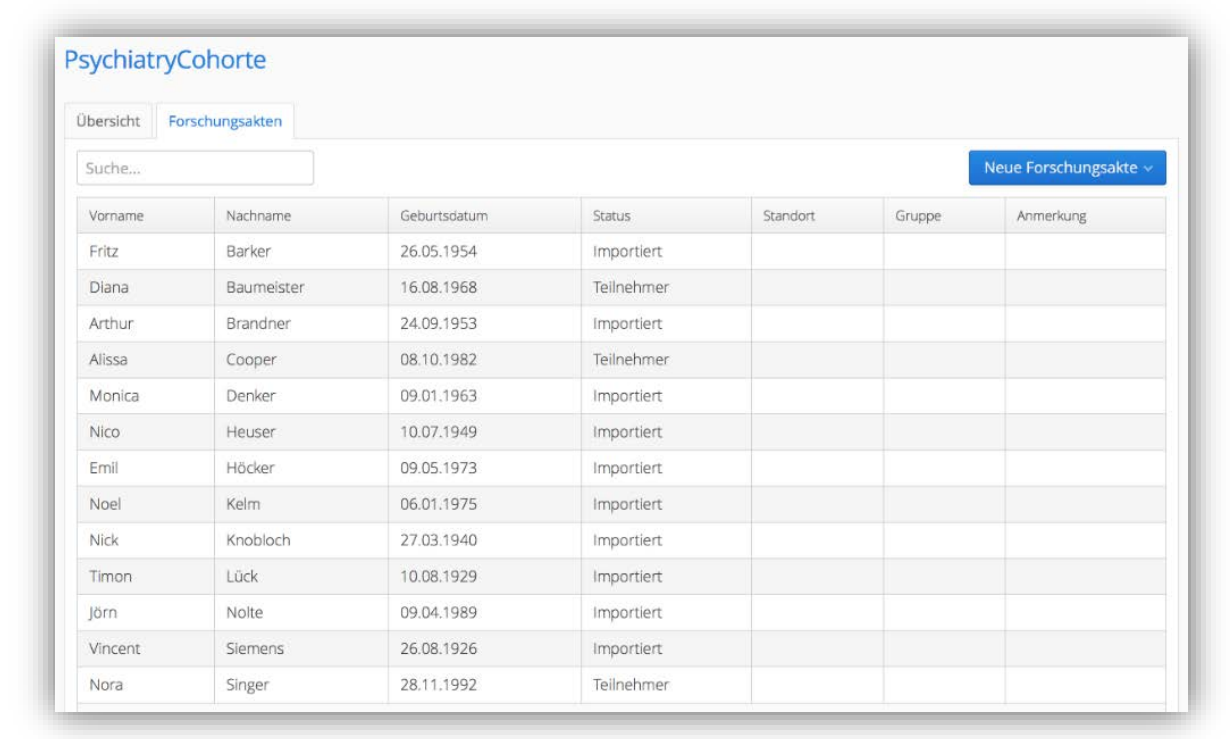

Abbildung 61. Importierte Beispieldaten im Probandenmanagementsystem

Die importierten Probandenstammdaten wurden mit dem Status „Importiert“ versehen.

Neben dem Import von Daten ist auch ein Export von Daten aus dem Probandenmanagementsystem, beispielsweise für Briefe, notwendig. Hierfür verfügt das Probandenmanagementsystem über eine rudimentäre Report-Engine. Ein Report umfasst eine SQL-Abfrage, deren Ergebnisse in einer Tabelle dargestellt und als Datei exportiert werden können. Abbildung 62 zeigt beispielhaft den Export aller zuvor importierten Probanden (A-F-19).

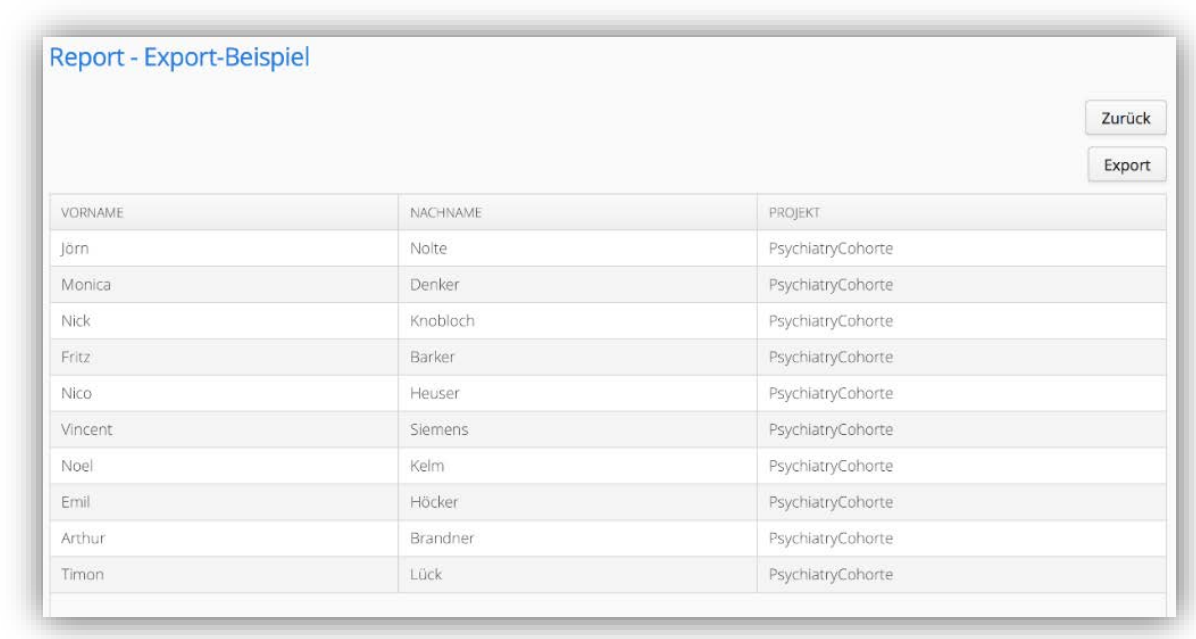

Abbildung 62. Export von Stammdaten aus dem Probandenmanagementsystem 


\subsection{Verwaltung von Einwilligungserklärungen}

Die Abbildung von Einwilligungserklärungen im Probandenmanagementsystem ist Gegenstand einer Abschlussarbeit, welche im Rahmen dieser Dissertation betreut wurde [118]. Aufgrund des umfangreichen Forschungsfeldes zum Thema Einwilligungserklärung wurde auf die Einarbeitung der Ergebnisse aus der Abschlussarbeit verzichtet. Folgerichtig werden die zugehörigen Anforderungen (A-F-20 bis A-F-24) daher durch den entwickelten Prototyp nicht erfüllt.

\subsection{Pseudonymisierung}

Die Pseudonymisierung von Probandenstammdaten ist ein wichtiger Aspekt in medizinischen Forschungsprojekten [47]. Daher war eine zentrale Anforderung an das Probandenmanagementsystem die Unterstützung der Pseudonymisierung. Die Verwaltung von Pseudonymen erfolgt in der bereits vorgestellten Forschungsakte eines Probanden. Dieser Reiter umfasst alle Pseudonyme eines Probanden innerhalb des jeweiligen Forschungsprojekts (siehe Abbildung 63).

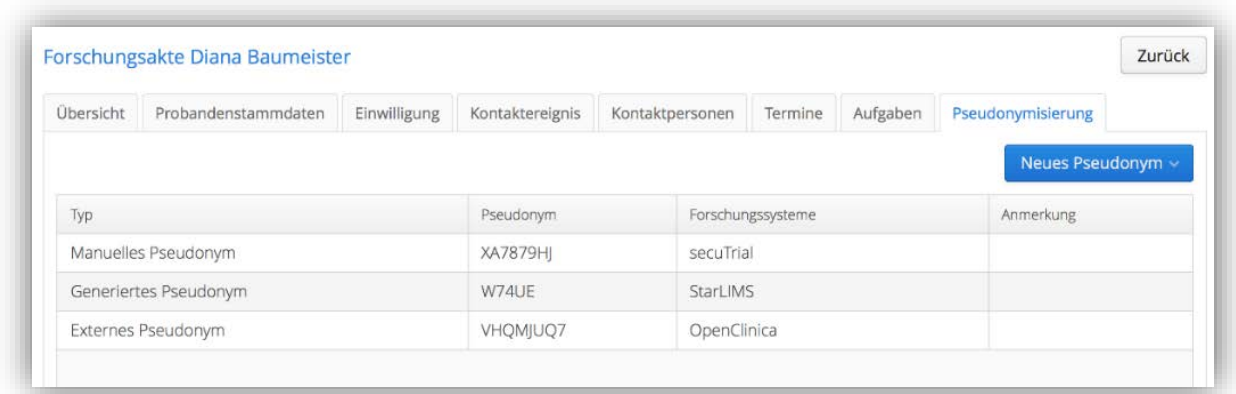

Abbildung 63. Reiter Pseudonymisierung in der Forschungsakte eines Probanden

Durch die Erfassung der Pseudonyme ist auch eine Suche nach einem Probanden anhand eines Pseudonyms (A-F-27) innerhalb eines Forschungsprojekts möglich (siehe Abbildung 64).

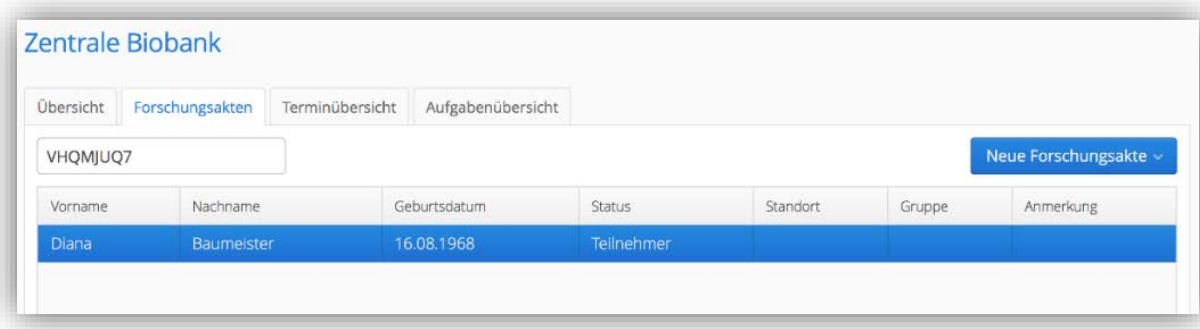

Abbildung 64. Suche nach Probanden anhand von Pseudonymen

Pseudonyme können, wie in Abbildung 65 dargestellt, entweder manuell eingegeben (A-F-25), automatisch generiert (A-F-28) oder von einem externen System abgerufen (A-F-29) werden. 


\section{Neues Pseudonym}

Manuelles Pseudonym

Generiertes Pseudonym

Externes Pseudonym

Abbildung 65. Dialog zur Auswahl, wie ein neues Pseudonym erstellt werden soll

Abbildung 66 und Abbildung 67 zeigen Beispielhaft den Abruf eines Pseudonyms von einem externen System (PIDGenerator).

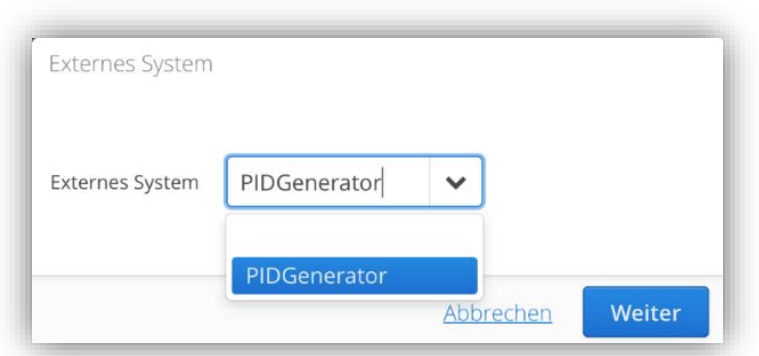

Abbildung 66. Dialog zum Abruf des Pseudonyms von einem externen System (PIDGenerator)

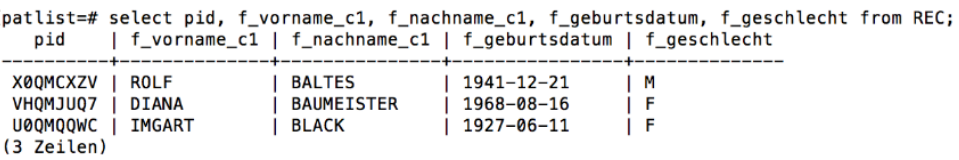

Abbildung 67. Zuordnung des Pseudonyms zu den Probandenstammdaten in der Datenbank des externen Systems

Nach dem Abruf von einem externen System folgt der gleiche Dialog wie bei der manuellen Eingabe oder bei Generierung eines Pseudonyms. In diesem Dialog (siehe Abbildung 68) kann der Benutzer die jeweiligen Forschungssysteme angeben, in welchen das Pseudonym genutzt wird (A-F-26).

Durch die Gegebenheit, dass Probanden mehrere Forschungsakten besitzen können, ist es möglich Pseudonyme projektübergreifend zuzuordnen. Diese Funktionalität wurde ebenfalls im Rahmen einer Abschlussarbeit erstellt [117]. Auf die Einarbeitung dieser Ergebnisse wurde auf Grund der Komplexität des Forschungsumfeldes verzichtet, daher wird die zugehörige Anforderung (A-F-30) folgerichtig als nicht erfüllt angesehen. 


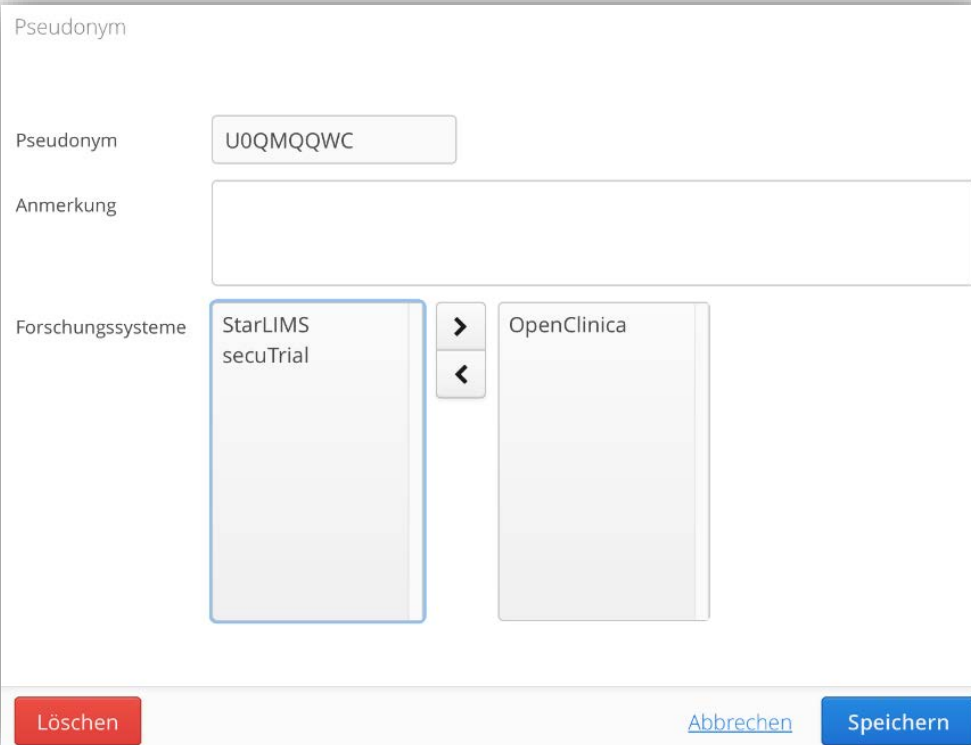

Abbildung 68. Dialog zur Dokumentation von Metadaten für ein Pseudonym

\subsection{Zusammenfassung}

Insgesamt wurden 23 der 30 funktionalen Anforderungen an das Probandenmanagementsystem durch den Prototyp erfüllt. Da die funktionalen Anforderungen mitunter aus den identifizierten Zielen und Szenarien gewonnen wurden, wird nachfolgend auch deren Erfüllung analysiert. In Tabelle 16 sind alle Ziele, deren zugehörige Szenarien sowie die daraus gewonnenen Anforderungen dargestellt.

Durch den Prototyp werden 13 der 16 Ziele erfüllt. Zu den nicht erfüllten Zielen gehört der Abruf von Probandenstammdaten aus SAP IS-H (Z-6), die Abbildung der Einwilligungserklärung (Z-12) sowie die Generierung von projektübergreifenden Forschungspseudonymen (Z16). Hieraus ergibt sich die Nichterfüllung bzw. nur teilweise Erfüllung von drei Szenarien (S2, S-8 und S-9). 


\begin{tabular}{|c|c|c|c|}
\hline Ziel & Szenario & Anforderung & Erfüllt \\
\hline $\begin{array}{l}\mathrm{Z}-1(\checkmark) \\
\mathrm{Z}-2(\checkmark)\end{array}$ & S-1 $(\checkmark)$ & $\begin{array}{l}\text { A-F-4 }(\checkmark) \\
\text { A-F-6 }(\checkmark) \\
\text { A-F-5 }(\checkmark) \\
\text { A-F-7 }(\checkmark) \\
\text { A-F-8 }(\checkmark) \\
\text { A-F-17 }(\checkmark)\end{array}$ & Ja \\
\hline $\begin{array}{l}\text { Z-3 }(\checkmark) \\
\text { Z-4 }(\checkmark) \\
\text { Z-5 }(\checkmark) \\
\text { Z-6 (x) } \\
\text { Z-7 }(\checkmark)\end{array}$ & S-2 (x) & $\begin{array}{l}\text { A-F-9 }(\checkmark) \\
\text { A-F-10 }(\checkmark) \\
\text { A-F-11 }(\checkmark) \\
\text { A-F-13 }(\mathbf{x}) \\
\text { A-F-14 }(\checkmark) \\
\text { A-F-15 }(\checkmark) \\
\text { A-F-16 }(\checkmark)\end{array}$ & Teilweise \\
\hline Z-8 ( $(\checkmark)$ & S-3 $(\checkmark)$ & $\begin{array}{l}\text { A-F-9 }(\sqrt{ }) \\
\text { A-F-11( }(\mathfrak{})\end{array}$ & $\mathrm{Ja}$ \\
\hline Z-9 $(\sqrt{ })$ & S-4 $(\checkmark)$ & $\begin{array}{l}\text { A-F-9 }(\checkmark) \\
\text { A-F-11 }(\checkmark) \\
\text { A-F-12 }(\checkmark)\end{array}$ & Ja \\
\hline $\mathrm{Z}-10(\checkmark)$ & S-5 $(\checkmark)$ & A-F-19 ( () & Ja \\
\hline $\mathrm{Z}-11(\vee)$ & S-6( $(\sqrt{ })$ & A-F-19 ( $\checkmark)$ & Ja \\
\hline Z-12 (x) & S-7 (x) & $\begin{array}{l}\text { A-F-20 (x) } \\
\text { A-F-21 (x) } \\
\text { A-F-22 (x) }\end{array}$ & Nein \\
\hline $\begin{array}{l}\text { Z-13 }(\checkmark) \\
\text { Z-14 }(\checkmark) \\
\text { Z-15 }(\checkmark)\end{array}$ & S-8 $(\checkmark)$ & $\begin{array}{l}\text { A-F-25 }(\checkmark) \\
\text { A-F-26 }(\checkmark) \\
\text { A-F-27 }(\checkmark) \\
\text { A-F-28 }(\checkmark)\end{array}$ & Ja \\
\hline Z-16 (x) & S-9(x) & A-F-30 (x) & nein \\
\hline
\end{tabular}

Tabelle 16. Dokumentation des Erfüllungsgrades der Ziele und Szenarien 


\section{Usability Test}

Ziel des Usability Tests war es zu untersuchen, ob das entwickelte Probandenmanagementsystem den Erwartungen der Stakeholder entspricht [128, 144]. Der Usability Test wurde mit einer Gruppe von zehn Stakeholdern durchgeführt. Alle Stakeholder hatten einen Bezug zur medizinischen Forschung sowie direkten Kontakt zu Probanden. Die Gruppe bestand aus zwei Wissenschaftlern, vier Ärzten sowie vier Studienassistenten.

Der Usability-Test umfasste eine Vorabbefragung, die Szenariobewertung, die Systembewertung sowie eine Vorschlagsliste für fehlende Systemfunktionen.

\subsection{Auswertung der Vorabbefragung}

In der Vorabbefragung gaben vier der zehn Stakeholder an, dass die Verwaltung ihrer Probanden über Excel-Tabellen erfolgt. Fünf Stakeholder nutzten bereits die Vorversion des Probandenmanagementsystems, welche die Grundlage dieser Dissertation bildete. Vor dem Einsatz des Systems nutzten die fünf Stakeholder Excel-Tabellen zur Verwaltung ihrer Probanden. Hierzu gehörten ein wissenschaftlicher Mitarbeiter (M), zwei Ärzte (A) und zwei Studienassistenten (S).

Um einen ersten Eindruck über positive oder negative Auswirkungen durch den Einsatz eines Probandenmanagementsystems in realen Forschungsprojekten zu erhalten, bewerteten die fünf Stakeholder die Vorversion des Probandenmanagementsystems (siehe Tabelle 17).

\begin{tabular}{l|l|l|l|l|l|l|l|l|l|l|l} 
& $\mathbf{F}_{\mathbf{1}}$ & $\mathbf{F}_{\mathbf{2}}$ & $\mathbf{F}_{\mathbf{3}}$ & $\mathbf{F}_{\mathbf{4}}$ & $\mathbf{F}_{\mathbf{5}}$ & $\mathbf{F}_{\mathbf{6}}$ & $\mathbf{F}_{\mathbf{7}}$ & $\mathbf{F}_{\mathbf{8}}$ & $\mathbf{F}_{\mathbf{9}}$ & $\mathbf{F}_{\mathbf{1 0}}$ & SUS \\
\hline Stakeholder 1 (A) & 1 & 2 & 4 & 2 & 2 & 3 & 4 & 2 & 1 & 1 & 55,0 \\
\hline Stakeholder 3 (A) & 2 & 1 & 5 & 2 & 5 & 1 & 5 & 1 & 4 & 1 & 87,5 \\
\hline Stakeholder 6 (S) & 5 & 1 & 5 & 1 & 5 & 2 & 5 & 1 & 3 & 1 & 92,5 \\
\hline Stakeholder 7 (S) & 3 & 1 & 5 & 1 & 4 & 1 & 5 & 1 & 5 & 1 & 92,5 \\
\hline Stakeholder 8 (M) & 5 & 1 & 5 & 1 & 4 & 1 & 5 & 1 & 5 & 1 & 97,5 \\
\hline \hline
\end{tabular}

Tabelle 17. Bewertung der Vorversion des Probandenmanagementsystems

Die Bewertung erfolgte unter Verwendung des System Usability Scale. Dessen Score ergab einen Median von 92,5. Dies bedeutet, dass das System für die Stakeholder nutzbar und dessen

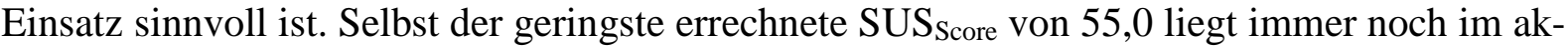
zeptablen Bereich (vgl. Abschnitt 2.5.2). Der höchste Wert für den SUS Score beträgt 97,5 und ist nah am Median.

\subsection{Szenariobewertung}

Zur Durchführung des Usability-Tests wurden fünf Szenarien entwickelt. Diese Szenarien dienen den Stakeholdern als Anleitung zur Interaktion mit dem Probandenmanagementsystem (siehe Anhang A.3, Szenario 1 bis 5). 


\subsubsection{Szenario 1}

Innerhalb des ersten Szenarios wurden die Stakeholder mit der Probandenübersicht vertraut gemacht. Diese bietet den Benutzern eine Liste mit allen Probanden sowie den Forschungsprojekten, denen die Probanden zugeordnet sind. In der Liste sind nur Probanden enthalten, die dem angemeldeten Benutzer zugeordnet sind. Insgesamt wurden die dargestellten Informationen durch die Stakeholder als nützlich und sinnvoll erachtet. Vier Stakeholder bewerteten dieses Szenario mit „stimme zu“ und sechs mit „stimme voll zu“.

Ein Stakeholder fände es sinnvoll, innerhalb der Übersicht auch eine Änderung der Probandenstammdaten zu ermöglichen. Zwei Stakeholder benötigten zur Erledigung ihrer täglichen Arbeit eine komplexere Suchfunktion, bspw. nach Forschungsprojekt, Geburtsdatum sowie einer Kombination aus verschiedenen Suchparametern. Ebenso wäre es für zwei Stakeholder wünschenswert, dass es möglich ist, zusätzliche Daten in die Übersicht aufzunehmen.

\subsubsection{Szenario 2}

Gegenstand des zweiten Szenarios bildet die projektübergreifende Verwaltung von Probanden. Dazu sollen die Stakeholder eine Forschungsakte für einen bereits erfassten Probanden anlegen. Eine mehrfache Eingabe von Stammdaten der Probanden soll so vermieden werden.

Die Stakeholder bewerteten diese Funktion positiv (sechs „stimme voll zu“, drei „stimme zu“ und ein „unentschlossen“). Bei Suche und Auswahl des Probanden sollten mehr Daten zu dem Probanden dargestellt werden. Insbesondere wurde die Effektivität und Schnelligkeit hervorgehoben.

\subsubsection{Szenario 3}

Das dritte Szenario umfasst die Erkennung von identischen Probanden anhand ihrer Stammdaten. Dies soll sicherstellen, dass ein Proband innerhalb des Systems nur einmal erfasst wird. Nach Eingabe der Stammdaten wird durch eine Duplikaterkennung geprüft, ob bereits ähnliche Stammdaten erfasst wurden. Falls die Ähnlichkeit sehr hoch ist, wird kein neuer Proband angelegt, sondern auf den existierenden Probanden verwiesen.

Insgesamt sahen die Stakeholder auch diese Funktion positiv. Sechs Stakeholder bewerteten die dargestellten Informationen als sinnvoll und nützlich mit „stimme voll zu“, drei mit „stimme zu“ und einer mit „unentschlossen“.

Ein großes Problem sahen die Stakeholder darin, dass kein deutlicher Hinweis darauf gegeben wurde, dass eine Duplikaterkennung erfolgte. Das System sollte den Benutzer darauf hinweisen, dass ein Proband mit ähnlichen Stammdaten bereits existiert und den Benutzer aktiv fragen, ob es sich um denselben Probanden handelt. 


\subsubsection{Szenario 4}

Im Vordergrund des vierten Szenarios stand die Integration des Probandenmanagementsystems in das Krankenhausinformationssystem zum Abruf von Probandenstammdaten. Das Anlegen eines neuen Probanden erfolgt unter Angabe der jeweiligen Patientenkennung. Anhand dieser Kennung werden die Stammdaten aus dem Krankenhausinformationssystem abgerufen. An der Universitätsmedizin Göttingen ist dies SAP IS-H.

Der Abruf von Probandenstammdaten wurde ebenfalls positiv bewertet. Sechs Stakeholder bewerteten die Funktion als sehr nützlich mit „stimme voll zu“, drei mit „stimme zu“ und ein Stakeholder mit „unentschlossen“. Ein Problem im Zusammenhang mit diesem Szenario war für die Stakeholder, dass eine nachträgliche Änderung der Stammdaten nicht direkt möglich ist. Generell wäre es seitens der Stakeholder wünschenswert, dass Stammdaten der Probanden durch sie selbst geändert werden können.

\subsubsection{Szenario 5}

Gegenstand des letzten Szenarios ist die Kommunikation mit einem externen System zur Pseudonymisierung von Probanden. Ein solches Szenario ist in multizentrischen Forschungsprojekten, wie bereits mehrfach angeführt, durchaus üblich. Die Stammdaten des Probanden werden entsprechend der Schnittstelle des externen Systems transformiert und nachfolgend an dieses übermittelt. Voraussetzung für die Übermittlung ist in jedem Fall die Einwilligungserklärung des Probanden.

Die Stakeholder erachteten die Kommunikation mit externen Systemen als sinnvoll und nützlich (fünf „stimme voll zu“, drei „stimme zu“ und ein „unentschlossen“). Bei der Durchführung des Szenarios kam es bei einem Stakeholder zu einem Systemfehler, welcher nicht rekonstruiert werden konnte. Daher wurde von diesem Stakeholder keine Bewertung abgegeben.

\subsection{Systembewertung}

Nach erfolgreicher Bearbeitung der fünf Szenarien bewerteten die zehn Stakeholder die Nutzbarkeit des entwickelten Probandenmanagementsystems. Die Systembewertung erfolgte unter Verwendung des System Usability Scale (siehe Tabelle 18).

Das entwickelte Probandenmanagementsystem wurde von den Stakeholdern gut bewertet. Der

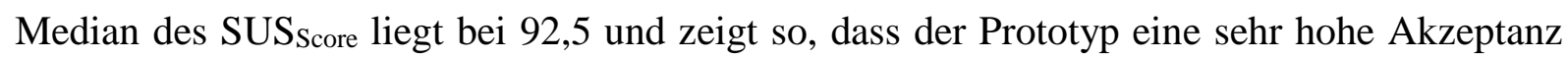
durch die Stakeholder aufweist. Dies wird noch einmal durch die beiden Quartile deutlich. Das 25\%-Quartil weist mit 84,4 einen Wert im hohen Akzeptanzbereich auf (vgl. Abschnitt 2.5.2). Darüber hinaus liegt das 75\%-Quartil mit 97,5 sehr nahe am Maximalwert von 100.

Neun der zehn Stakeholder konnten sich vorstellen, das Probandenmanagementsystem nach dessen Einführung häufig zu nutzen (F1). Dies lag vor allem an der geringen Komplexität des Systems (F2) sowie der einfachen Benutzbarkeit (F3). Ebenso waren die Stakeholder der Meinung, dass sie keine Hilfe bei der Nutzung des Systems benötigen (F4). 


\begin{tabular}{l|l|l|l|l|l|l|l|l|l|l|l} 
& $\mathbf{F}_{\mathbf{1}}$ & $\mathbf{F}_{\mathbf{2}}$ & $\mathbf{F}_{\mathbf{3}}$ & $\mathbf{F}_{\mathbf{4}}$ & $\mathbf{F}_{\mathbf{5}}$ & $\mathbf{F}_{\mathbf{6}}$ & $\mathbf{F}_{\mathbf{7}}$ & $\mathbf{F}_{\mathbf{8}}$ & $\mathbf{F}_{\mathbf{9}}$ & $\mathbf{F}_{\mathbf{1 0}}$ & SUSscore \\
\hline Stakeholder 1 (A) & 5 & 2 & 4 & 2 & 2 & 3 & 4 & 2 & 1 & 1 & 65,0 \\
\hline Stakeholder 2 (A) & 4 & 2 & 4 & 2 & 3 & 2 & 4 & 1 & 3 & 1 & 75,0 \\
\hline Stakeholder 3 (A) & 5 & 1 & 5 & 4 & 5 & 2 & 5 & 1 & 4 & 1 & 87,5 \\
\hline Stakeholder 4 (M) & 3 & 2 & 5 & 1 & 4 & 1 & 5 & 1 & 5 & 1 & 90,0 \\
\hline Stakeholder 5 (S) & 5 & 2 & 5 & 1 & 4 & 2 & 4 & 1 & 5 & 1 & 90,0 \\
\hline Stakeholder 6 (S) & 5 & 1 & 5 & 1 & 5 & 1 & 5 & 1 & 4 & 2 & 95,0 \\
\hline Stakeholder 7 (S) & 5 & 1 & 5 & 1 & 5 & 1 & 4 & 1 & 5 & 1 & 97,5 \\
\hline Stakeholder 8 (M) & 5 & 1 & 5 & 1 & 5 & 1 & 5 & 1 & 4 & 1 & 97,5 \\
\hline Stakeholder 9 (S) & 5 & 1 & 5 & 1 & 5 & 1 & 5 & 1 & 4 & 1 & 97,5 \\
\hline Stakeholder 10 (A) & 4 & 1 & 5 & 1 & 5 & 1 & 5 & 1 & 5 & 1 & 97,5 \\
\hline \hline & & & & & & & & & & &
\end{tabular}

Tabelle 18. Bewertung des entwickelten Probandenmanagementsystems

Aus Perspektive der Stakeholder waren alle durch die Szenarien dargestellten Funktionen sehr gut im System integriert (F5) und enthielten darüber hinaus wenige Inkonsistenzen (F6). Die Stakeholder waren sich sicher, dass auch andere Personen den Umgang mit dem System leicht erlernen können (F7). Dies lag zum einen daran, dass das System nicht umständlich zu nutzen ist, und zum anderen fühlten sich acht der zehn Stakeholder bei der Benutzung des Systems sehr sicher (F9). Ebenso bestand keine sehr hohe Lernkurve zur Nutzung des Probandenmanagementsystems (F10).

Zusammenfassend zeigt die Systembewertung, dass das entwickelte Probandenmanagementsystem den Ansprüchen der Stakeholder entspricht und zur Erfüllung ihrer Anforderungen geeignet erscheint. Jedoch bewertet ein Stakeholder (Stakeholder 1) die Usability des entwickelten Systems geringer als die anderen Stakeholder. Der gleiche Stakeholder bewertete auch die Usability der Vorversion des Probandenmanagementsystems geringer (siehe Tabelle 17). Da beide Bewertungen der Usability immer noch im akzeptablen Bereich liegen, wurde keine nähere Untersuchung durchgeführt. Die Kommentare des Stakeholders im Validierungsprotokoll zeigen, dass er ein Probandenmanagementsystem wünscht, welches mehrere Einrichtungen umfasst und nicht nur die Universitätsmedizin Göttingen.

\subsection{Vorschlagsliste für Systemfunktionen}

Zum Abschluss des Usability-Tests wurden die Stakeholder gebeten, fehlende Funktionen des Probandenmanagementsystems zu nennen. Eine wichtige Erweiterung für sie wäre eine verbesserte Suchfunktion. Diese sollte über den Namen hinaus weitere Suchparameter enthalten und auch komplexe Abfrage ermöglichen, bspw. eine Kombination aus Status, Geburtsdatum und Name. Oftmals ergeben sich die Suchanfragen aus aktuellen Situationen, sodass ein Kollektiv von Probanden anhand wechselnder Merkmale identifiziert werden muss, beispielsweise für Briefe oder zur Rekrutierung für neue Forschungsprojekte. 
Ebenso wäre es seitens der Stakeholder wünschenswert, dass zusätzliche Merkmale bzw. Metadaten zu den einzelnen Forschungsprojekten und Probanden erfasst werden können. Auf Grundlage dieser zusätzlichen Merkmale sollte ebenfalls eine Suche möglich sein. Eng in diesem Zusammenhang steht auch der Wunsch nach einer umfassenden Aufgaben- und Terminplanung. Typischerweise folgen Untersuchungen in einem Forschungsprojekt einer festgelegten Reihenfolge von Aufgaben, die für alle Probanden durchgeführt werden müssen. Dies ist meist mit einzelnen Visiten bzw. Terminen verbunden. Um die durchgeführten Aufgaben in Zusammenhang mit den Probanden besser zu dokumentieren, sollte das Informationssystem zum Probandenmanagement nach Ansicht der Stakeholder eine Funktion zur Verwaltung von Aufgaben und Terminen bieten. 


\section{Diskussion}

In Deutschland besteht großer Bedarf an einer effizienten und einheitlichen Verwaltung sowie einer langfristigen Nachverfolgung von Probanden in medizinischen Forschungsprojekten [1, 2]. Innerhalb dieser Dissertation wurde daher untersucht, inwieweit die Etablierung eines Probandenmanagementsystems den Bedarf an Effizienz, Einheitlichkeit und Langfristigkeit deckt.

Der Aspekt Effizienz beschreibt in dieser Dissertation die Verwaltung einer Vielzahl von Probanden sowie die enge Verbindung zwischen der Identität des Patienten im Krankenhaus und der des Probanden in Forschungsprojekten [5-7, 16-18]. Im Zusammenhang mit dem Aspekt der Einheitlichkeit steht die immer stärker werdende Vernetzung der deutschen Gesundheitsforschung. Diese Vernetzung führt dazu, dass einzelne Forscher einer Einrichtung ihre Probanden in verschiedenen Forschungsprojekten verwalten müssen [2, 22, 36, 37, 47, 49]. Dies erfolgt typischerweise durch unterschiedliche Softwaresysteme, welche an die spezifischen Bedürfnisse der einzelnen Forschungsprojekte angepasst sind [41, 44, 47, 54-59]. Eine einheitliche Verwaltung der Probanden ist daher nicht gegeben. Der letzte Aspekt umfasst die langfristige Nachverfolgung von Probanden. Dies ist vor allem für Biobanken wichtig, da diese eine erneute Kontaktierung von Probanden sicherstellen müssen und zum anderen den Probanden aufzeigen wollen, in welchen Forschungsprojekten ihre Daten und Biomaterialien genutzt werden $[65,68]$. Ebenso muss die Nachverfolgbarkeit von Probanden bei Forschungsprojekten im Bereich von chronischen Erkrankungen über mehrere Forschergenerationen sichergestellt werden [70].

Die Untersuchung, inwieweit die Etablierung eines Probandenmanagementsystems den Bedarf an Effizienz, Einheitlichkeit und Nachhaltigkeit deckt, wurde anhand zweier Forschungsfragen am Beispiel der Universitätsmedizin Göttingen durchgeführt. Im nachfolgenden Abschnitt 8.1 wird die Beantwortung der Forschungsfragen zusammenfassend erläutert.

In Abschnitt 8.2 wird das methodische Vorgehen in dieser Dissertation im Ganzen kritisch analysiert und hinterfragt. Die drauffolgenden Abschnitte richten den Fokus der Diskussion auf die Anforderungsanalyse (Abschnitt 8.3), das Architekturkonzept (Abschnitt 8.4), die prototypische Implementierung (Abschnitt 8.5) sowie die Validierung in Form einer Funktionsprüfung (Abschnitt 8.6) und eines Usability Tests (Abschnitt 8.7). Den Abschluss der Diskussion bildet die Einordnung des Probandenmanagements im Forschungsumfeld (Abschnitt 8.8), der Einsatz des Probandenmanagements an der Universitätsmedizin Göttingen (Abschnitt 8.9) sowie ein Ausblick auf weitere Entwicklungen und Forschungsfelder (Abschnitt 8.10).

\subsection{Zusammenfassung}

Der Ausgangspunkt dieser Dissertation war der Bedarf von Forschern an einer einheitlichen und effizienten Verwaltung sowie langfristigen Nachverfolgung von Probanden. Aus diesem Bedarf wurden zwei Forschungsfragestellungen abgeleitet: Innerhalb der ersten Forschungs- 
frage wurden Szenarien für den Einsatz des zu konzeptionierenden Probandenmanagementsystems an der Universitätsmedizin Göttingen identifiziert. Die zweite Forschungsfrage sollte untersuchen, wie ein Probandenmanagementsystem unter Berücksichtigung der identifizierten Szenarien und der vorhandenen Schnittstellen von externen Systemen konzipiert und prototypisch implementiert werden kann.

Zur Beantwortung der ersten Forschungsfragestellung wurden zunächst Ziele identifiziert, welche durch das zu konzeptionierende Probandenmanagementsystem erfüllt werden sollten. Ziele beschreiben die Intention von Stakeholdern und bilden die Grundlage zur Gewinnung von Szenarien [99-104]. Die Identifizierung der Ziele erfolgte unter Anwendung von leitfadengestützten Interviews, die mit 17 Mitarbeitern der Universitätsmedizin Göttingen (Stakeholder) durchgeführt wurden. Alle Interviews wurden aufgezeichnet und mit Hilfe der qualitativen Inhaltsanalyse ausgewertet. Insgesamt wurden so 16 Ziele identifiziert, welche in die Bereiche „Verwaltung von Forschungsprojekten“, „Verwaltung von Probanden“ und „Pseudonymisierung“ unterteilt wurden.

Die 16 Ziele bildeten wiederum die Grundlage zur Ermittlung der Szenarien. Ein Szenario beschreibt die Interaktion eines Akteurs (Benutzer oder System) mit dem Probandenmanagementsystem zur Erfüllung eines oder mehrerer Ziele. Beginnend mit dem ersten Ziel wurde ein Szenario zur Erfüllung dieses Ziels modelliert. Für das nächste Ziel wurde geprüft, ob es ebenfalls durch ein existierendes Szenario - ggf. durch Anpassung - erfüllt wird. Falls nein, wurde ein neues Szenario modelliert. Auf Grundlage dieses Vorgehens wurden neun Szenarien zur Beantwortung der ersten Forschungsfragestellung modelliert.

Gegenstand der zweiten Forschungsfrage war die Konzeption und prototypische Implementierung eines Probandenmanagementsystems unter Berücksichtigung der Szenarien und vorhandenen Schnittstellen. Ausgangspunkt für die Konzeption des Systems bildeten lösungsorientierte Anforderungen. Diese wurden aus den identifizierten Zielen und Szenarien, den Stakeholderinterviews sowie den Ergebnissen einer Literaturrecherche gewonnen. Die gewonnenen Anforderungen wurden in drei Arten unterteilt: funktionale Anforderungen, Qualitätsanforderungen und Randbedingungen. Insgesamt wurden 30 funktionale Anforderungen, sieben Qualitätsanforderungen und neun Randbedingungen identifiziert. Die funktionalen Anforderungen wurden zur besseren Strukturierung in fünf Kategorien unterteilt: Authentifizierung und Autorisierung, Verwaltung von Forschungsprojekten, Verwaltung von Probanden, Verwaltung von Einwilligungserklärungen und Pseudonymisierung.

Die identifizierten 46 lösungsorientierten Anforderungen waren der Ausgangspunkt zur Entwicklung des Architekturkonzepts. Grundlage hierfür bildete das Modell des Domain-Driven Design. Bei dessen Anwendung steht die Fachlichkeit der Softwareanwendung im Vordergrund (Fachdomäne) und nicht die technische Umsetzung. Die Fachlichkeit des Probandenmanagementsystems wurde mittels UML-Diagrammen modelliert und wird als Domänenmodell bezeichnet. Innerhalb des Domain-Driven Design bildet das Domänenmodell die Fachlichkeit und 
den Kern des Systems [120, 121]. Die Zugangspunkte zu dem Domänenmodell, beispielsweise für eine Benutzeroberfläche, werden durch die primären Schnittstellen beschrieben. Relevante technische Aspekte, wie z. B. der Datenbankzugriff oder die Kommunikation mit externen Systemen, werden durch die sekundären Schnittstellen abstrahiert. Im Architekturkonzept konnten 24 der 30 funktionalen Anforderungen berücksichtigt werden sowie zwei Qualitätsanforderungen.

Der nächste Schritt zur Beantwortung der zweiten Forschungsfrage war die prototypische Implementierung des Architekturkonzepts. Da eine Validierung des Prototyps durch zukünftige Benutzer des Systems angedacht war, wurde der Prototyp in Form eines Pilotsystems entwickelt. Ein Pilotsystem verfügt über eine hohe Qualität und kann nachfolgend als Grundlage für ein produktives System genutzt werden. Die Entwicklung des Prototyps erfolgte in der Programmiersprache Java unter Verwendung von industriell erprobten Softwarebibliotheken. Hierdurch konnte zum einen die Entwicklungszeit reduziert und zum anderen die Qualität erhöht werden.

In der nachfolgenden Funktionsprüfung, als Teil der Validierung, wurde untersucht, welche lösungsorientierten Anforderungen, Szenarien und Ziele durch den Prototyp erfüllt werden. Durch den entwickelten Prototyp werden 23 der 30 funktionalen Anforderungen, zwei Qualitätsanforderungen sowie vier Randbedingungen erfüllt. Die nicht erfüllten funktionalen Anforderungen umfassen die Verwaltung von Einwilligungserklärungen, die Generierung von Forschungspseudonymen sowie den Abruf von Patientenstammdaten aus dem klinisch-administrativen System des Krankenhauses. Letzteres wurde auf Grund der komplexen Schnittstellenlandschaft im Krankenhaus nicht implementiert. Sowohl der Bereich Einwilligungserklärung als auch die Generierung von Forschungspseudonymen wurden durch zwei Abschlussarbeiten im Forschungsumfeld dieser Dissertation bearbeitet [117, 118]. Aufgrund des umfangreichen Forschungsumfelds wurden die Ergebnisse der Arbeiten nicht in diese Dissertation aufgenommen. Daher wurden die zugehörigen Anforderungen folgerichtig als nicht erfüllt angesehen. Aus diesem Umstand ergibt sich, dass nur 13 von 16 Zielen und sechs von neun Szenarien vollständig erfüllt werden. Ein Szenario wird teilweise erfüllt, da bis auf ein zugeordnetes Ziel alle durch den Prototyp abgebildet werden. Die verbleibenden Randbedingungen und Qualitätsanforderungen sind organisatorischer Art und können daher nicht durch den Prototyp erfüllt werden, beispielsweise dass der Betrieb des Systems im gesicherten Intranet zu erfolgen hat.

Neben der Funktionsprüfung bildete ein Usability Test den zweiten Teil der Validierung. Der Usability Test wurde mit zehn Stakeholdern durchgeführt. Ziel des Tests war es, die Nutzbarkeit des Systems aus Sicht der Stakeholder bewerten zu lassen. Generell zeigten die Ergebnisse des Tests eine sehr hohe Bewertung. Grundlage waren fünf Szenarien, welche aus den neun identifizierten Szenarien abgeleitet wurden. Alle Szenarien wurden durch die Stakeholder selbst mit dem Prototyp des Probandenmanagementsystems bearbeitet. Innerhalb des ersten Szenarios sollten die Stakeholder mit dem System vertraut gemacht werden und die Probandenübersicht kennenlernen. Gegenstand des zweiten, dritten und vierten Szenarios war das Anlegen eines 
Probanden in einem Forschungsprojekt. Dabei wurde den Stakeholdern die Duplikaterkennung gezeigt, die Übernahme von Probanden aus vorhandenen Forschungsprojekten sowie der Abruf von Stammdaten aus dem klinisch-administrativen des Systems des Krankenhauses. Dieser Abruf wurde durch eine Mock-Implementierung umgesetzt, da die Implementierung im Prototyp auf Grund der Komplexität nicht realisiert wurde. Das letzte Szenario umfasste die Pseudonymisierung von Probanden durch ein externes System. Dies ist ein typisches Verfahren in multizentrischen medizinischen Forschungsprojekten [41, 47]. Insgesamt bewerteten die Stakeholder sowohl die Abbildung der Szenarien als auch das System sehr positiv. Einschränkend wurde von den Stakeholdern die zentrale Verwaltung von Probanden und die damit verbundene Restriktion zur Änderung und Löschung angesehen. Ebenso fehlte den Stakeholdern eine Terminund Visitenplanung zur besseren Koordination der Studienvisiten innerhalb der Forschungsprojekte. Dennoch wünschten sich alle Stakeholder, das entwickelte Probandenmanagementsystem zeitnah für ihre Forschungsprojekte nutzen zu können.

\subsection{Methodisches Vorgehen}

Das methodische Vorgehen, welches dieser Dissertation zu Grunde liegt, orientiert sich an dem klassischen Softwareentwicklungszyklus nach dem Wasserfallmodell [114]. Zunächst wurden Anforderungen an das Probandenmanagementsystem identifiziert. Auf Grundlage dieser Anforderungen entstand das Architekturkonzept. Dieses wurde nachfolgend implementiert und durch eine Funktionsprüfung sowie einen Usability Test validiert.

Generell weist das Wasserfallmodell einige Schwächen auf: So kann innerhalb des Wasserfallmodells nicht flexibel auf neue Anforderungen eingegangen werden, da diese sehr früh definiert werden. Die Aufnahme einer neuen Anforderung würde dazu führen, dass der bisherige Prozess unterbrochen und wieder mit der Anforderungsanalyse begonnen werden muss. Eine weitere Herausforderung ist, dass das System erst sehr spät den Stakeholdern bzw. potentiellen Benutzern zur Verfügung gestellt wird. Dies bedeutet, dass fehlende Anforderungen oder Systemfehler erst spät erkannt werden können.

Im Bereich der Softwareentwicklung werden daher agile Vorgehensmodelle präferiert [114]. Innerhalb der agilen Softwareentwicklung stehen die Menschen im Vordergrund und nicht die Prozesse. So binden die agilen Vorgehensmodelle die Stakeholder mehr in die Entwicklung des Systems ein. Zudem sind inkrementelle Auslieferungen der zu implementierenden Systeme vorgesehen. Hierdurch können die Stakeholder zeitnah bereits mit den Systemen arbeiten und Änderungswünsche mitteilen. [114]

Innerhalb dieser Dissertation musste auf ein agiles Vorgehensmodell verzichtet werden. Dies lag vor allem an der hohen Arbeitsbelastung der Stakeholder, sodass mehrere Termine zur Diskussion von Anforderungen sowie Systemtests nur schwer möglich gewesen wären. Generell wäre ein agiles Vorgehensmodell sehr empfehlenswert. So wären zunächst im Rahmen eines initialen Workshops die Funktionsbereiche des Probandenmanagementsystems identifiziert 
worden. Beispielsweise bestünden die Funktionsbereiche aus Projektverwaltung, Probandenverwaltung und Pseudonymisierung. Unter enger Einbindung der Stakeholder wären dann für den ersten Bereich die notwendigen Funktionen spezifiziert worden. Ein Teil dieser Funktionen wäre dann implementiert und durch die Stakeholder getestet worden. Die fehlenden Funktionen, aus Sicht der Stakeholder, wären dann Gegenstand der nächsten Implementierungsphase gewesen.

Ein solches Vorgehen erfordert eine zeitnahe, meist wöchentliche Abstimmung mit Stakeholdern. Dies wäre im Rahmen dieser Dissertation auf Grund der Arbeitsbelastung der Stakeholder kaum möglich gewesen. Rückblickend war die gewählte Vorgehensweise basierend auf dem Wasserfallmodell eine gute Wahl. Die Stakeholder wurden nur zwei Mal eingebunden, wodurch die extreme Arbeitsbelastung berücksichtigt werden konnte. Ebenso zeigte der durchgeführte Usability Test, dass der entwickelte Prototyp von den Stakeholder durchaus als nutzbar angesehen wird. Durch die ziel- und szenariobasierten Analyse standen die Intentionen der Stakeholder im Vordergrund und nicht die technischen Anforderungen an das Probandenmanagementsystem.

\subsection{Anforderungsanalyse}

Ziel der Anforderungsanalyse war die Identifizierung von Szenarien zur Beantwortung der ersten Forschungsfrage sowie die Gewinnung von Anforderungen zur Konzeption des Probandenmanagementsystems. Grundlage der Anforderungsanalyse bildete daher die ziel- und szenariobasierte Analyse. Im ersten Schritt wurden zunächst Ziele des konzeptionierenden Systems identifiziert, welche dann in einem zweiten Schritt zur Gewinnung von Szenarien verwendet werden. Im letzten Schritt wurden aus den Zielen und Szenarien die lösungsorientierten Anforderungen hergeleitet. Durch das verwendete Vorgehensmodell konnten die Szenarien sowie die benötigten Anforderungen identifiziert werden [99].

Selbstverständlich wäre die Identifizierung der Szenarien und Anforderungen auch unabhängig voneinander möglich gewesen. In diesem Fall wäre aber eine nachträgliche Zuordnung der Szenarien zu den Anforderungen zur besseren Nachvollziehbarkeit notwendig gewesen. Ebenso wäre auf die Identifizierung von Zielen verzichtet worden; dieses Vorgehen stellt jedoch eine bewährte Methode zur Gewinnung von Szenarien dar [99-104].

Die Ziele beschreiben die Intentionen von Stakeholdern und wurden durch leitfadengestützte Interviews gewonnen. Die Durchführung von Interviews bot sich an, da durch diese die Intention und Erwartung der Stakeholder an ein Probandenmanagementsystem erfasst werden konnte [99, 108]. Es wurden 17 Stakeholder, welche unterschiedliche Rollen an der Universitätsmedizin Göttingen haben, interviewt. Alle Stakeholder sind Kooperationspartner des Instituts für Medizinische Informatik an der Universitätsmedizin Göttingen. 
Durch die 17 Interviews konnte ein erstes Meinungsbild über das Probandenmanagement erfasst werden. Jedoch bedarf die Einführung eines Informationssystems an einem Universitätsklinikum der Berücksichtigung von deutlich mehr Stakeholdern [108], wie beispielsweise Vertretern des Vorstands, Institutsdirektoren sowie einem breiteren Spektrum an Mitarbeitern. Da der Aufwand zur Durchführung und Analyse der Interviews hoch ist, würde sich beispielsweise die Durchführung eines Workshops mit verschiedenen Stakeholdergruppen anbieten [99]. Dies ist zwar ebenfalls aufwendig, jedoch könnten mehr Stakeholder berücksichtigt werden [99, 108].

Die Auswertung der Interviews zur Identifizierung der Ziele erfolgte unter Anwendung der qualitativen Inhaltsanalyse. Diese kann auch angewendet werden, wenn keine vollständige Transkription der Interviews erfolgt und ist ein etabliertes Verfahren in den Sozialwissenschaften [110-112]. Eine direkte Frage nach Zielen wäre in den Interviews mitunter nicht zielführend gewesen, da hierdurch eher funktionale Anforderungen in den Vordergrund gerückt wären. Zur Qualitätssicherung wäre es sinnvoll gewesen, die identifizierten Ziele mit den jeweiligen Stakeholdern nochmals abzustimmen. Aufgrund der hohen Arbeitsbelastung der Stakeholder musste auf dieses Vorgehen verzichtet werden. Um dennoch qualitätsgesicherte Aussagen in Form von Zielen aus den Interviews zu gewinnen, wurde die qualitative Inhaltsanalyse angewendet. Insgesamt wurden so 16 Ziele identifiziert. Die nachfolgende Kategorisierung der Ziele zeigte, dass im Bereich des Probandenmanagements vor allem die Verwaltung von Forschungsprojekten, die Verwaltung von Probanden und die Pseudonymisierung wichtige Themen aus Sicht der Stakeholder sind.

Im nächsten Schritt der ziel- und szenariobasierten Analyse wurden aus den 16 Zielen die zugehörigen Szenarien hergeleitet. Ein Szenario beschreibt die Interaktion eines Akteurs (Benutzer oder System) mit dem Probandenmanagementsystem zur Erfüllung eines oder mehrerer Ziele [99-101]. Die Entwicklung der Szenarien ermöglicht, die abstrakten Ziele in eine, auch für die Stakeholder nachvollziehbare Beschreibung zu überführen. Aus den 16 Zielen wurden insgesamt neun Szenarien abgeleitet. Eine direkte Modellierung der Szenarien aus den Interviews heraus bot sich nicht an, da die Grundlage eines Szenarios immer die Erfüllung mindestens eines Ziels ist. Die Szenarien wurden nur im Hinblick auf die Zielerfüllung modelliert, möglich wären auch negative Szenarien oder Missbrauchsszenarien [99]. Grundsätzlich ist es empfehlenswert auch diese Szenarien zu modellieren um mögliche Probleme zu identifizieren. Gemeinsam mit den Szenarien zur Zielerfüllung bilden sie dann einen Anwendungsfall ab [99, 108]. Da diese nicht Gegenstand der ersten Forschungsfragestellung waren, wurde auf deren Modellierung verzichtet. Im Idealfall wäre nach der Modellierung der Szenarien eine erneute Abstimmung mit den Stakeholdern notwendig gewesen. Auf diese musste ebenfalls auf Grund der Arbeitsbelastung der Stakeholder verzichtet werden.

Im letzten Schritt der Anforderungsanalyse wurden aus den 16 Zielen und neun Szenarien konkrete Anforderungen an das Probandenmanagementsystem gewonnen [99, 103]. Neben den 
Zielen und Szenarien dienten als weitere Quellen zur Gewinnung die zusammengefassten Interviews sowie die Ergebnisse der durchgeführten Literaturrecherche. Innerhalb der Dissertation wurde zwischen drei verschiedenen Arten von lösungsorientierten Anforderungen unterschieden: funktionale Anforderungen, Qualitätsanforderungen und Randbedingungen [99]. Auf die typische Unterteilung in funktionale und nicht-funktionale Anforderungen wurde verzichtet, da nicht-funktionale Anforderungen entweder unterspezifizierte funktionale Anforderungen oder Qualitätsanforderungen sind [99].

Insgesamt wurden 46 lösungsorientierte Anforderungen an das Probandenmanagementsystem gewonnen: 30 funktionale Anforderungen, sieben Qualitätsanforderungen und neun Randbedingungen. Interessant waren zwei Qualitätsanforderungen (A-Q-3) und (A-Q-4): Ein Probandenmanagementsystem sollte den Regularien der Good Clinical Practice (GCP) und Good Automated Manufacturing Practice (GAMP) entsprechen. Diese Regularien enthalten Vorschriften zur Entwicklung und Validierung eines Informationssystems im Rahmen von klinischen Studien nach Arzneimittelgesetz [156]. Beide Qualitätsanforderungen wurden nicht weiter berücksichtigt, da der Gegenstand dieser Dissertation nur die prototypische Implementierung war und nicht der Einsatz des Systems im Umfeld von klinischen Studien nach Arzneimittelgesetz. Sollte das Probandenmanagementsystem in diesem Umfeld zum Einsatz kommen, so ist die Umsetzung von GCP und GAMP empfehlenswert, dies erfordert jedoch ein umfangreiches Team aus Softwareentwicklern und Qualitätsmanagern [156].

In der Publikation Schwanke et al. [1] wurden deutlich mehr Anforderungen an ein Probandenmanagementsystem identifiziert. Dies liegt an dem sehr weiten Fokus der Publikation. Innerhalb dieser Dissertation standen die effiziente und einheitliche Verwaltung sowie die langfristige Nachverfolgung von Probanden im Vordergrund. So wurde beispielsweise die Termin- und Visitenplanung (Dispositionsmanagement) in Schwanke et al. [1] als Anforderung genannt. Jedoch ist dieser Bereich derart breit gefächert, dass die Termin- und Visitenplanung separat zu untersuchen ist. Gleiches gilt auch für das Forschungsumfeld zum Thema Einwilligungserklärung und projektübergreifende Forschungspseudonyme. Beide Bereiche wurden jeweils in einer Abschlussarbeit untersucht [117, 118], welche im Rahmen dieser Dissertation betreut wurde. Daher wurden die Bereiche in Form von Zielen, Szenarien und lösungsorientierten Anforderungen zur Vollständigkeit in die Dissertation aufgenommen.

Generell stehen die identifizierten Ziele, Szenarien und lösungsorientierten Anforderungen in engem Zusammenhang mit den jeweiligen Stakeholdern. Daher sind die gewonnenen Ergebnisse der Anforderungsanalyse nicht per se auf andere Einrichtungen übertragbar. Würde beispielsweise an einer Universitätsklink eine Verfahrensbeschreibung zur Verwaltung von Probanden existieren, wäre sicherlich ein Ziel diese im Probandenmanagementsystem abzubilden. Generell zeigen aber die Beispiele aus der Einleitung, dass in der deutschen medizinischen Forschung durchaus ein Bedarf an einem Probandenmanagementsystem existiert. Um eine sichere Übertragbarkeit der Ergebnisse der Anforderungsanalyse sicherzustellen, wären weitere Unter- 
suchungen notwendig. Ein mögliches methodisches Vorgehen würde darin bestehen, die Ergebnisse durch andere Einrichtungen zu verifizieren. Die identifizierten Ziele, Szenarien und Anforderungen würden im Rahmen von mehreren Workshops an verschiedenen Einrichtungen vorgestellt und mit den vorhandenen Erwartungen der dortigen Stakeholder abgeglichen. Nach einer Konsolidierungsphase würden dann die finalen Ergebnisse der Anforderungsanalyse den Stakeholdern im Rahmen eines Abschlussworkshops gemeinsam vorgestellt. Nur ein solches Vorgehen kann die Übertragbarkeit der Ergebnisse fundiert belegt werden. Die identifizierten Ziele, Szenarien und lösungsorientierten Anforderungen bilden daher eine gute Grundlage für weitere Untersuchungen im Hinblick auf die Übertragbarkeit.

\subsection{Architekturkonzept}

Gemäß der zweiten Forschungsfragestellung wurde untersucht, wie ein Probandenmanagementsystem unter Berücksichtigung der identifizierten Szenarien und Schnittstellen der externen Systeme konzeptioniert und prototypisch implementiert werden kann.

Externe Systeme sind in diesem Zusammenhang Softwaresysteme zur Pseudonymisierung von Probanden in medizinischen Verbundforschungsprojekten. In diesen Projekten wird typischerweise ein zentrales System betrieben, welches die Generierung von Pseudonymen unter Angabe von Probandenstammdaten ermöglicht [41, 47].

Grundlage zur Entwicklung des Architekturkonzepts bildeten die gewonnenen 46 lösungsorientierten Anforderungen an das Probandenmanagementsystem. Bei der Modellierung des Architekturkonzepts sollte die Abbildung der Anforderungen im Vordergrund stehen und nicht die technische Umsetzung des Systems. Daher wurde zur Modellierung des Architekturkonzepts das von Eric Evans eingeführte Konzept des Domain-Driven Design angewendet [121]. Im Vordergrund steht dabei die Lösung der Problemstellung (Fachdomäne), für welche das System entwickelt wird und nicht die eigentliche Entwicklung des Systems. Die Problemstellung wurde durch die identifizierten Ziele, Szenarien und lösungsorientierten Anforderungen repräsentiert [97].

Das auf dem Domain-Driven Design basierende Architekturkonzept ähnelt einer klassischen Schichtenarchitektur, führt aber zu einer strikteren Trennung der Schichten. Die Grundlage hierfür bildet der Architekturstil Ports and Adapters [120, 122]. Dieser unterteilt die Architektur in ein Domänenmodell, primäre und sekundäre Schnittstellen. Das Domänenmodell bildet die eigentliche Problemstellung ab und wird durch die primären Schnittstellen zur Verwendung bereitgestellt. So kann beispielsweise eine graphische Benutzeroberfläche über die primären Schnittstellen auf das Domänenmodell zugreifen. Die technischen Details werden durch die sekundären Schnittstellen abstrahiert, beispielsweise den Datenbankzugriff oder die Kommunikation mit externen Systemen. Durch den verwendeten Ansatz des Domain-Driven Design wird die Softwarearchitektur verständlicher und nachvollziehbarer [120]. 
Theoretisch wäre auch ein vollständiger Verzicht auf eine Softwarearchitektur möglich gewesen. Direkt nach der Spezifikation der lösungsorientierten Anforderungen hätte die Entwicklung des Probandenmanagementsystems begonnen. Durch den Verzicht auf eine Softwarearchitektur wäre das zugrundeliegende System kaum nachvollziehbar und nur schwer erweiterbar gewesen [119, 120]. Eine Übertragbarkeit der Ergebnisse wäre folglich ausgeschlossen. Daher wurde auf eine direkte Implementierung selbstverständlich verzichtet und eine umfassende Softwarearchitektur entworfen und dokumentiert.

Das entwickelte Architekturkonzept besteht aus sieben Bereichen: Systemkonfiguration, Sicherheit, Projektverwaltung, Probandenverwaltung, Forschungsakte, Kommunikation und Pseudonymisierung. Insgesamt umfasst das Domänenmodell des Architekturkonzepts 27 Entities, drei Value Objects und drei Aggregates. Neun primäre Schnittstellen wurden zur direkten Verwendung der Fachdomäne in Form von Services modelliert. Diese nutzen neun Repositories, vier Provider sowie neun spezifische Implementierungen der Provider durch geeignete Adapter. Durch das entwickelte Architekturkonzept werden 24 der 30 funktionalen Anforderungen berücksichtigt sowie zwei Qualitätsanforderungen (A-Q-6) und (A-Q-7) bereits erfüllt. Damit wurde eine sehr gute Ausgangslage zur Implementierung des Prototyps für ein Probandenmanagementsystem geschaffen.

Die sechs funktionalen Anforderungen, welche nicht berücksichtigt wurden, waren Gegenstand von zwei Abschlussarbeiten [117, 118]. Innerhalb beider Abschlussarbeiten wurde das entwickelte Architekturkonzept dieser Dissertation verwendet, sodass eine Integration der Ergebnisse durchaus möglich ist.

Kern des entwickelten Architekturkonzepts ist die Forschungsakte. Diese ordnet Probanden den jeweiligen Forschungsprojekten zu und ist vergleichbar mit dem Konzept der Fallakte im Krankenhaus [71]. Alle forschungsspezifischen Daten zu einem Probanden werden über die Forschungsakte erfasst, dies gilt auch für Einwilligungserklärungen. Die Forschungsakte ist ein wesentlicher Bestandteil des Systems und ermöglicht die zentrale Verwaltung von Probanden über mehrere Forschungsprojekte. Grundsätzlich wäre es auch möglich gewesen, für jede Teilnahme eines Probanden an einem Forschungsprojekt eine neue Instanz des Probanden anzulegen. So wären eine projektübergreifende Verwaltung sowie ein Datenabgleich aber nur mit sehr hohem Aufwand über eine Metaebene möglich gewesen.

Das Architekturkonzept wurde primär für ein Probandenmanagementsystem an der Universitätsmedizin Göttingen entwickelt. Um eine Übertragbarkeit auf andere Universitätskliniken zu ermöglichen, können die bisherigen sekundären Schnittstellen durch neue Implementierungen ersetzt werden. Hierdurch kann das System an eine neue Betriebsumgebung angepasst werden, ohne die Kernfunktionalität zu ändern. Sicherlich führt die Definition und Implementierung von sekundären Schnittstellen zu einem höheren Entwicklungsaufwand. Jedoch wird durch die sekundären Schnittstellen die Übertragbarkeit auf andere Betriebsumgebungen vereinfacht. 


\subsection{Prototypische Implementierung}

Das Ziel der prototypischen Implementierung bestand darin, die Umsetzbarkeit des Architekturkonzepts zu untersuchen sowie einen Prototyp für die Validierung des Probandenmanagementsystems durch eine Gruppe von Stakeholdern bereitzustellen.

Die Nutzung von Prototypen ist eine bewährte Methode im Bereich der Softwareentwicklung [114, 128]. Beispielsweise werden Prototypen verwendet um Stakeholdern einen ersten Eindruck des Systems zu ermöglichen [99, 108]. Es ist durchaus möglich, dass ein Prototyp Teil des späteren Systems wird. Dieser Prototyp wird dann oftmals als Pilotsystem bezeichnet [128].

Da der Prototyp nicht nur zur Untersuchung der Umsetzbarkeit des Architekturkonzepts dient, sondern auch zur Validierung genutzt werden soll, muss er eine entsprechend hohe Qualität und Funktionalität aufweisen, sodass eine Nutzung durch die Stakeholder möglich ist. Daher erfolgte die Entwicklung des Probandenmanagementsystems in Form eines Pilotsystems, das als Grundlage für die spätere produktive Verwendung dient (siehe Abschnitt 8.9).

Der entwickelte Prototyp wurde vollständig in Java implementiert. Die Programmiersprache Java wurde ausgewählt, da prominente Softwareanwendungen im Bereich der medizinischen Forschung ebenfalls auf Grundlage von Java entwickelt wurden [130-134].

Um eine möglichst hohe Qualität des Prototyps zu erreichen, wurden etablierte Softwarebibliotheken verwendet. Diese werden auch im industriellen Bereich zur Entwicklung von unternehmenskritischen Anwendungen eingesetzt [137, 138, 140, 141]. Ein weiterer Vorteil, der durch die Verwendung der Softwarebibliotheken entstand, war die Reduzierung des Implementierungsaufwands. Zum Beispiel ermöglichte die Bibliothek Hibernate eine effiziente Abbildung des Domänenmodells auf eine relationale Datenbank. Durch die Softwarebibliothek Spring wurden die benötigten Repositories des Architekturkonzepts automatisch zur Verfügung gestellt.

Das Ergebnis der prototypischen Implementierung war eine Java-basierte Webanwendung, welche das Architekturkonzept vollständig umsetzt. Durch die verwendete Softwarebibliothek Vaadin war es möglich die web-basierte Benutzeroberfläche vollständig in der Programmiersprache Java zu entwickeln [141].

Durch die Verwendung des Ports and Adapters Architekturstils und der Softwarebibliothek Spring können die Adapter einfach durch andere Adapter ausgetauscht werden. So ist das entwickelte Probandenmanagementsystem nicht nur in der Universitätsmedizin Göttingen, sondern auch in anderen Gesundheitseinrichtungen einsetzbar. 


\subsection{Funktionsprüfung}

Im Anschluss an die prototypische Implementierung des Probandenmanagementsystems wurde als Teil der Validierung eine Funktionsprüfung vorgenommen. Grundlage hierfür bildeten die identifizierten 30 funktionalen Anforderungen sowie der entwickelte Prototyp.

Die Funktionsprüfung wurde durchgeführt, um nachvollziehbar darzustellen, welche gestellten Anforderungen durch den Prototyp erfüllt werden. Die funktionalen Anforderungen wurden einzeln im Hinblick auf die Erfüllung untersucht und durch Screenshots belegt. Zur Sicherstellung der Nachvollziehbarkeit der Funktionsprüfung ist dieser Dissertation der Quellcode des Prototyps inklusive einer Testumgebung als Anhang auf DVD beigefügt (siehe Anhang A.7).

Der verwendete Prototyp basiert auf dem entwickelten Architekturkonzept, verfügt aber über weitere Funktionen. Diese zusätzlichen Funktionen wurden im Rahmen der Konsolidierung und Übergabe des Prototyps an das Institut für Medizinische Informatik der Universitätsmedizin Göttingen ergänzt (siehe Abschnitt 8.9). Daher waren diese Funktionen nicht Gegenstand der Funktionsprüfung, wurden aber teilweise in den Screenshots dargestellt.

Durch den Prototyp werden 23 der 30 funktionalen Anforderungen erfüllt. Die nicht erfüllten Anforderungen umfassen den Abruf von Probandenstammdaten aus dem klinisch-administrativen System, die Verwaltung von Einwilligungserklärungen sowie die Generierung von projektübergreifenden Forschungspseudonymen. Auf Grund der komplexen Schnittstellenlandschaft wurde auf den Abruf von Probandenstammdaten verzichtet. Typischerweise erfolgt die Kommunikation von Stammdaten im Krankenhaus über HL7-Nachrichten [71]. Die notwendigen Nachrichten hätten entsprechend implementiert und gegen den Kommunikationsserver der Universitätsmedizin Göttingen getestet werden müssen. Zum einen wäre die Implementierung aufwendig gewesen, und zum anderen wären Personalressourcen im Rechenzentrum benötigt worden. Daher wurde auf die Umsetzung des Abrufs von Stammdaten verzichtet. Die notwendige sekundäre Schnittstelle wurde jedoch im Architekturkonzept berücksichtigt.

Die Verwaltung von Einwilligungserklärungen sowie die Generierung von projektübergreifenden Forschungspseudonymen waren Gegenstand von zwei Abschlussarbeiten. Beide Abschlussarbeiten wurden bereits im Architekturkonzept auf Grund des umfangreichen Forschungsfeldes nicht berücksichtigt. Daher erfolgte auch keine Umsetzung der Anforderungen im Rahmen der prototypischen Implementierung.

Durch die gewählten Softwareframeworks konnten neben den funktionalen Anforderungen auch vier Randbedingungen erfüllt werden: Konzeption als Webanwendung, kein Einsatz zusätzlicher Software zum Betrieb notwendig, Unterstützung mehrerer Sprachen sowie Erfassung aller Änderungen an den Datensätzen. Die verbleibenden nicht erfüllten Randbedingungen und Qualitätsanforderungen sind organisatorischer Art und können daher nicht durch eine Funktionsprüfung untersucht werden. 
Unter Berücksichtigung der erfüllten bzw. nicht erfüllten Anforderungen wurden 13 der 16 identifizierten Ziele erfüllt. Die nicht erfüllten Ziele stehen im direkten Zusammenhang mit dem Abruf von Stammdaten, der Abbildung von Einwilligungserklärungen und den übergreifenden Forschungspseudonymen.

Ausgehend von der Zielerfüllung werden sechs der neun Szenarien vollständig durch den Prototyp umgesetzt. Ein Szenario wurde nur teilweise umgesetzt, da eines der sechs zugehörigen Ziele nicht erfüllt wurde. Generell wäre eine vollständige Erfüllung der Ziele, Szenarien und Anforderungen möglich gewesen, jedoch mit einem extrem hohen Aufwand. Da es sich bei dem entwickelten Probandenmanagementsystem explizit um einen Prototyp handelt, ist eine vollständige Erfüllung nicht unbedingt notwendig.

\subsection{Usability Test}

Ziel des Usability Tests war es zu untersuchen, ob das prototypisch entwickelte Probandenmanagementsystem den Erwartungen der Stakeholder entspricht [128]. Dies konnte nur geprüft werden, indem die Stakeholder das Probandenmanagementsystem aktiv nutzten. Da ein freies Experimentieren mit dem System teilweise zu subjektiven Einschätzungen führt [99], wurde ein Usability-Test mit fünf Szenarien entwickelt. Die Szenarien des Usability-Tests sollten die Stakeholder anleiten und so die subjektiven Einschätzungen reduzieren. Grundlage der Szenarien bildeten die neun identifizierten Szenarien der ziel- und szenariobasierten Analyse. Sie beschreiben typische Arbeitsabläufe, welche die Stakeholder mit dem entwickelten Prototyp durchführen. Nach jedem Szenario sollten die Stakeholder dieses kurz bewerten.

Nach Durchführung der fünf Szenarien sollten die Stakeholder den Prototyp zusammenfassend bewerten. Hierfür wurde der System Usability Scale verwendet [144]. Dieser umfasst zehn Fragen und bietet die Möglichkeit, einfach und schnell einen ersten Anhaltspunkt für die Usability des Systems zu ermitteln [144, 145, 151]. Es existieren weitere Fragebögen zur Ermittlung der Usability eines Systems [144]; da dies jedoch nicht der zentrale Aspekt dieser Dissertation war, wurde ein einfach und schnell durchzuführender Test - der System Usability Scale - verwendet.

Bereits fünf Stakeholder sind ausreichend um einen ersten Eindruck von der Usability einer Softwareanwendung zu erhalten [148]. In der Literatur wird die Durchführung eines UsabilityTests mit ca. zehn Stakeholdern empfohlen [147-149]. Hierdurch soll ein Großteil der Probleme der Softwareanwendung identifiziert werden. Die Auswahl der Stakeholder erfolgte auf Grundlage der durchgeführten Interviews. Da nicht alle der 17 interviewten Stakeholder zukünftige Nutzer des Probandenmanagementsystems sind, wurden weitere sechs Stakeholder aus der Universitätsmedizin Göttingen in den Usability-Test einbezogen. Die Auswahl der zusätzlichen Stakeholder erfolgte aus den Projektpartnern des Instituts für Medizinische Informatik, die zukünftige Nutzer des Probandenmanagementsystems sein könnten. Insgesamt waren vier Ärzte, zwei wissenschaftliche Mitarbeiter und vier Studienassistenten an der Validierung des Systems beteiligt. 
Vor dem eigentlichen Usability-Test wurde eine kurze Vorabbefragung durchgeführt. Ziel war es, zusätzliche Informationen über Stakeholder zu erhalten, z. B. welche Rolle der Stakeholder innehat, oder wie die Probanden der Stakeholder aktuell verwaltet werden. Dabei zeigte sich, dass die Probandenverwaltung meist über eine Excel-Tabelle erfolgt. Fünf der zehn Stakeholder nutzten eine Vorversion des entwickelten Probandenmanagementsystems. Die Vorversion ist nur für ein einzelnes Forschungsprojekt ausgelegt und verfügt über keine projektübergreifende Verwaltung. Dennoch wird eine ähnliche Zielsetzung verfolgt, daher wurden die Stakeholder gebeten, die genutzte Vorversion zu bewerten. Hierdurch sollte ein erstes Stimmungsbild über den Einsatz eines Informationssystems zum Probandenmanagement in Forschungsprojekten erfasst werden. Die Vorversion erhielt eine positive Bewertung durch die fünf Stakeholder (Median von 92,5); dies zeigte, dass der Einsatz des Systems durchaus sinnvoll und nützlich ist.

Ein ähnliches Ergebnis zeigte auch die Bewertung der fünf Szenarien durch die zehn Stakeholder. Die dargestellten Informationen sowie deren zugrundeliegenden Systemfunktionen wurden von den Stakeholdern durchgehend positiv bewertet. Gegenstand des ersten Szenarios war die projektübergreifende Sicht auf alle dem Benutzer zugeordneten Probanden. Die nachfolgenden drei Szenarien umfassten das Anlegen neuer Probanden bzw. deren Einschluss in Forschungsprojekte. Dies erfolgte durch Auswahl eines vorhandenen Probanden, Eingabe der Stammdaten sowie Abruf der Stammdaten aus dem Krankenhausinformationssystem. Kritisch wurde in diesem Zusammenhang bewertet, dass eine Änderung der Stammdaten nicht direkt durch die Benutzer erfolgen kann, sondern nur durch den Administrator. Diese Einschränkung wurde gemacht, da ein Proband an mehreren Forschungsprojekten beteiligt sein kann und so dessen Stammdaten von verschiedenen Benutzern genutzt werden. Eine mögliche Lösung wäre, das Rechte- und Rollenmanagement entsprechend anzupassen, sodass die Probandenstammdaten durch die Benutzer geändert werden können, solange der Proband nur einem Forschungsprojekt zugeordnet ist. In diesem Zusammenhang müsste die Duplikaterkennung von ähnlichen Probanden berücksichtigt und gegebenenfalls angepasst werden.

Das fünfte Szenario umfasste die Vernetzung des Probandenmanagementsystems mit einem externen System zur Pseudonymisierung. In multizentrischen Forschungsprojekten ist der Einsatz eines zentralen Systems zur Pseudonymisierung das empfohlene Vorgehen, um einheitliche und standortübergreifende Pseudonyme zu erzeugen [41, 47]. Vor allem die Stakeholder, welche in Verbundprojekten aktiv sind, bewerteten diese Funktion positiv. Hierdurch können ihre Probanden von einem System aus projektübergreifend verwaltet werden. Generell wird den Stakeholdern so eine einheitliche Verwaltung ihrer Probanden ermöglicht.

Die abschließende Systembewertung zeigte, dass der Prototyp bereits eine hohe Nutzerzufriedenheit aufweist. Der Median des System Usability Scale, der zur Systembewertung genutzt wurde, hat einen Wert von 92,5. Dieser Wert spiegelt die hohe Akzeptanz des Systems durch die Nutzer wider [151]. Selbst das 25\%-Quartil liegt mit 84,4 deutlich im akzeptablen Bereich [151]. In diesem Zusammenhang ist auch besonders hervorzuheben, dass neun Stakeholder sich 
vorstellen konnten, das System häufig zu benutzen. Dies lag zum einen an der geringen Komplexität und zum anderen an der Einfachheit des Systems. Ebenso war die Nutzung des Systems nach Ansicht der Stakeholder mit einer geringen Einarbeitungszeit verbunden. Die meisten Stakeholder fühlten sich bei der Benutzung des Systems sicher. Auch waren die einzelnen Systemfunktionen des Prototyps gut integriert.

Ein Stakeholder zeigte eine deutliche Abweichung bei der Bewertung der Vorversion als auch bei dem entwickelten Prototypen. Hintergrund ist, dass der Stakeholder in seinen Forschungsprojekten ein Probandenmanagementsystem benötigt, welches mehrere Einrichtungen umfasst und nicht nur die Universitätsmedizin Göttingen. Da seine Bewertung jedoch immer noch im akzeptablen Bereich lag, wurde keine weitere Analyse vorgenommen. Prinzipiell existieren zwei Möglichkeiten um die Anforderungen des Stakeholders abzubilden. Die erste Möglichkeit erfordert keinerlei Implementierungsaufwand, da die vorhandene Projektverwaltung als Standortverwaltung genutzt wird. Ein Standort wird dann durch ein Projekt repräsentiert unter der Prämisse, dass das System nur für ein Forschungsprojekt genutzt wird. Im Rahmen der zweiten Möglichkeit müsste die vorhandene Fachdomäne um eine Standortverwaltung erweitert werden. Einzelne Projekte im Probandenmanagement würden dann Standorten zugeordnet werden. Dies ermöglicht eine generischere Verwendung des Systems im Hinblick auf die Wiederverwendbarkeit.

Die Systemfunktionen des Prototyps wurden im Hinblick auf eine effiziente und einheitliche Verwaltung sowie eine langfristige Nachverfolgung von Probanden über mehrere Forschungsprojekte modelliert und entwickelt. Daher wurden die Stakeholder zum Abschluss des Usability-Tests gebeten, fehlende Systemfunktionen zu nennen. Die Erfassung zusätzlicher Metadaten über Probanden und Forschungsprojekte, eine detaillierte Suchfunktion sowie eine Aufgaben- und Terminplanung wurden als wichtige fehlende Funktionen seitens der Stakeholder angesehen. Im Verlauf der Weiterentwicklung des Prototyps des Probandenmanagementsystems sollten daher auch diese Funktionen in das System aufgenommen werden.

Zusammenfassend konnte durch die Validierung gezeigt werden, dass die Erwartungen der Stakeholder an ein Probandenmanagementsystem erfüllt wurden. Nach Abschluss des UsabilityTests fragten alle Stakeholder, ob und wann sie das System in Zukunft nutzen könnten. Diese Frage verdeutlichte nochmals, dass ein großer Bedarf an einem Probandenmanagementsystem an der Universitätsmedizin Göttingen besteht und das entwickelte System durchaus diesen Bedarf deckt.

\subsection{Einordnung des Probandenmanagements im Forschungsumfeld}

In der durchgeführten Literaturrecherche (siehe Abschnitt 1.3), welche den aktuellen Stand der Technik und Forschung untersuchte, wurde gezeigt, dass bereits Systeme im Umfeld des Probandenmanagements existieren. Die existierenden Systeme sind jedoch nicht als Konkurrenz zum entwickelten System zu sehen, sondern als Ergänzung. Dies wird nachfolgend für die drei identifizierten Schwerpunkte der Literaturrecherche erläutert. 
Innerhalb des ersten Schwerpunkts Sekundärnutzung bietet sich das Probandenmanagementsystem als Vermittler zwischen Versorgung und Forschung an. Es ordnet der Identität des Patienten in der Versorgung die Identität des Probanden in der Forschung zu. Zudem bietet das System die Möglichkeit, alle Forschungsprojekte einer Gesundheitseinrichtung zu erfassen. Durch die Angabe geeigneter Metadaten ist so auch eine Suche nach geeigneten Forschungsprojekten für Patienten als Probanden möglich. Hierfür müsste ein geeigneter Standard ausgewählt und entsprechend im Probandenmanagementsystem ergänzt werden, beispielsweise der Standarddatensatz des Deutschen Registers Klinischer Studien [160].

Innerhalb des zweiten Schwerpunkts Pseudonymisierung könnte das Probandenmanagementsystem die einheitliche Verwaltung und Pseudonymisierung über verschiedene vorhandene Systeme erleichtern. Dies ist einer der wichtigsten Aspekte der Konzeption und Entwicklung des Systems. Durch den modularen Aufbau ist es einfach möglich, weitere Systeme bspw. zur zentralen Pseudonymisierung anzubinden.

Im Schwerpunkt Prozessmanagement könnte das entwickelte Probandenmanagementsystem als Ergänzung zu bestehenden Studienmanagementsystemen fungieren. So könnte das Probandenmanagementsystem beispielsweise das Studienmanagementsystem über neue Probanden informieren und gleichzeitig das Pseudonym übermitteln. Ebenso wäre auch eine Synchronisation von Visiten und Terminen zwischen den beiden Systemen denkbar.

\subsection{Einsatz des Probandenmanagementsystems}

Die Konzeption und Entwicklung des Probandenmanagementsystems erfolgte am Beispiel der Universitätsmedizin Göttingen. Diese wurde gewählt, da die Dissertation im Forschungsumfeld des Instituts für Medizinische Informatik der Universitätsmedizin Göttingen entstand. Durch den modularen Aufbau ist auch ein Einsatz des Systems in einer anderen Gesundheitseinrichtung möglich. Es müssten lediglich die Adapter, welche für die Universitätsmedizin Göttingen entwickelt wurden, angepasst werden.

In der Validierung zeigte sich, dass auch die Stakeholder (Forscher) durchaus Interesse an der Nutzung des entwickelten Probandenmanagementsystems haben. Jedoch erfordert der Einsatz des Systems eine grundlegende Änderung der Einwilligungsklärung: Jeder Proband muss zustimmen, dass seine personenbezogenen Daten, welche in dem jeweiligen Forschungsprojekt an der Universitätsmedizin Göttingen erhoben werden, zentral durch die Universitätsmedizin Göttingen verwaltet werden. Eine solche Änderung erfordert die Zustimmung der Ethikkommission, des behördlichen Datenschutzbeauftragten und gegebenenfalls des Landesdatenschutzbeauftragten. Da dies ein aufwendiges juristisches Verfahren darstellt, wurde folgende Zwischenlösung entwickelt: Für die sekundäre Schnittstelle zur Duplikaterkennung, welche die projektübergreifende Verwaltung sicherstellt, wurde ein neuer Adapter implementiert. Dieser Adapter schränkt die projektübergreifende Verwaltung der Probanden ein, sodass eine Dupli- 
katerkennung nur innerhalb eines Forschungsprojekts möglich ist. So ist die projektübergreifende Verwaltung von Probanden zwar möglich, aber deaktiviert. Hierdurch ist keine Anpassung der bestehenden Einwilligungserklärung notwendig.

Im Rahmen der Übergabe des entwickelten Probandenmanagementsystems an das Institut für Medizinische Informatik wurde das System um weitere Funktionen wie beispielsweise Terminund Aufgabenplanung erweitert. In diesem Zusammenhang wurde die bestehende Vorversion des Probandenmanagementsystems abgelöst. Das entwickelte Probandenmanagementsystem befindet sich seit Juni 2015 in drei Forschungsprojekten an der Universitätsmedizin Göttingen im produktiven Einsatz.

\subsection{Ausblick}

Der Einsatz des entwickelten Probandenmanagementsystems in weiteren Forschungsprojekten an der Universitätsmedizin Göttingen erfordert lediglich die Konfiguration eines neuen Projekts im System. Eine große Herausforderung stellt jedoch der Einsatz des Probandenmanagementsystems in klinischen Studien dar, die den Regularien der GCP und der GAMP unterliegen. Das Dokumentations- und Testniveau für Systeme in klinischen Studien ist deutlich höher als für andere Systeme [156].

Eine potentielle Möglichkeit zur Reduzierung des anfallenden Arbeitsaufwandes für Dokumentation und Testung besteht im Konzept des Continuous Delivery. Der Begriff Continuous Delivery beschreibt eine Sammlung von Techniken zur Automatisierung und Verbesserung der Auslieferung von Softwareanwendungen [136]. Continuous Delivery ermöglicht die Entwicklung qualitativ hochwertiger und verlässlicher Softwareanwendungen durch umfassende Testautomatisierung und automatische Aktualisierung der Software auf den Anwendungsservern [136]. Die Verwendung von Continuous Delivery im Umfeld von GCP und GAMP ist ein innovativer Ansatz und sollte im Rahmen einer wissenschaftlichen Arbeit analysiert werden.

Weitere wissenschaftliche Arbeiten sollten die Nutzung des Probandenmanagementsystems in großen Verbundforschungsprojekten untersuchen. Ein Forschungsverbund verbindet einzelne Gesundheitseinrichtungen in einem speziellen Themenbereich der Medizin; ein Beispiel hierfür wäre das Deutsche Zentrum für Herz-Kreislauf-Forschung. An die Stelle der Gesundheitseinrichtung tritt der Forschungsverbund. In diesem Zusammenhang müsste auch die Kommunikation zwischen dem zentralen Probandenmanagementsystem und den jeweiligen lokalen Systemen der Gesundheitseinrichtungen untersucht werden. Im Vordergrund der Untersuchung sollte stehen, wie Daten zwischen dem zentralen und den lokalen Systemen synchronisiert werden können.

In diesem Zusammenhang wäre es auch überlegenswert die Telematikinfrastruktur als Konnektor für das Probandenmanagement zu nutzen. Die Telematikinfrastruktur dient zur Verbindung zwischen verschiedenen medizinischen Leistungserbringern in Deutschland auf Grundlage ei- 
ner elektronischen Patientenakte [77]. Krister Helbing beispielsweise untersuchte in seiner Dissertation, wie diese Infrastruktur für die Forschung genutzt werden könnte. Helbing betrachtete die Nutzung von Daten aus der elektronischen Patientenakte in der medizinischen Forschung und nicht das Probandenmanagement. Problematisch ist in diesem Kontext die enge gesetzliche Fassung der Telematikinfrastruktur, die keine Nutzung durch die medizinische Forschung vorsieht [77]. Durch einen neuen Gesetzentwurf für sichere digitale Kommunikation und Anwendungen im Gesundheitswesen (E-Health-Gesetz) soll die Telematikinfrastruktur und die damit verbundene Patientenakte für die Forschung geöffnet werden [161]. Die erste Lesung des Gesetzentwurfs erfolgte am 03. Juli 2015 im deutschen Bundestag [162]. Sollte es zu einer Gesetzesänderung kommen, wäre eine wissenschaftliche Untersuchung zur Entwicklung eines Konnektors für das Probandenmanagement möglich.

Im Rahmen dieser Dissertation konnte gezeigt werden, dass das entwickelte Probandenmanagementsystem Forschern eine effiziente und einheitliche Verwaltung sowie langfristige Nachverfolgung ihrer Probanden über mehrere Forschungsprojekte ermöglicht. Derzeit befindet sich das Probandenmanagementsystem in drei Forschungsprojekten an der Universitätsmedizin Göttingen im Einsatz, um Effizienz, Einheitlichkeit und langfristige Nachverfolgbarkeit im Bereich des Probandenmanagements sicherzustellen. 


\section{Literaturverzeichnis}

[1] Schwanke J, Rienhoff O, Schulze TG, Nussbeck SY. Suitability of Customer Relationship Management Systems for the Management of Study Participants in Biomedical Research. Methods of Information in Medicine 2013; 52(4):340-350.

[2] IT-Reviewing-Board der TMF. IT-Infrastrukturen in der patientenorientierten Forschung: Aktueller Stand und Handlungsbedarf 2014. Berlin: Aka-Verlag; 2015.

[3] Sullivan PF. The Psychiatric GWAS Consortium: Big Science Comes to Psychiatry. Neuron 2010; 68(2):182-186.

[4] Visscher PM, Brown MA, McCarthy MI, Yang J. Five Years of GWAS Discovery. American Journal of Human Genetics 2012; 90(1):7-24.

[5] Anderson-Schmidt H, Adler L, Aly C, Anghelescu I, Bauer M, Baumgärtner J et al. The "DGPPN-Cohort": A National Collaboration Initiative by the German Association for Psychiatry and Psychotherapy (DGPPN) for Establishing a large-scale Cohort of Psychiatric Patients. European Archives of Psychiatry and Clinical Neuroscience 2013; 263(8):695-701.

[6] Lango Allen H, Estrada K, Lettre G, Berndt SI, Weedon MN, Rivadeneira F et al. Hundreds of Variants Clustered in Genomic Loci and Biological Pathways Affect Human Height. Nature 2010; 467(7317):832-838.

[7] Demiroglu SY, Skrowny D, Quade M, Schwanke J, Budde M, Gullatz V et al. Managing Sensitive Phenotypic Data and Biomaterial in large-scale Collaborative Psychiatric Genetic Research Projects: Practical Considerations. Molecular Psychiatry 2012; 17(12):1180-1185.

[8] Nationaler Aktionsplan für Menschen mit Seltenen Erkrankungen: Handlungsfelder, Empfehlungen und Maßnahmenvorschläge. Bonn; 2013.

http://www.bmg.bund.de/fileadmin/dateien/Downloads/N/NAMSE/Nationaler_Aktionsplan_fuer_Menschen_mit_Seltenen_Erkrankungen_-_Handlungsfelder_Empfehlungen_und_Massnahmenvorschlaege.pdf (11.10.2015).

[9] Maßnahmen zur Verbesserung der gesundheitlichen Situation von Menschen mit seltenen Erkrankungen in Deutschland: Forschungsbericht. Berlin; 2009. http://www.bmg.bund.de/fileadmin/dateien/Downloads/Forschungsberichte/110516_Forschungsbericht_Seltene_Krankheiten.pdf (11.10.2015).

[10] Pommerening K, Debling D, Kaatsch P, Blettner M. Register zu seltenen Krankheiten: Patientencompliance und Datenschutz. Bundesgesundheitsblatt, Gesundheitsforschung, Gesundheitsschutz 2008; 51(5):491-499.

[11] Rückinger S, Boneberger A. Epidemiologische Herausforderungen bei seltenen Krankheiten. Bundesgesundheitsblatt, Gesundheitsforschung, Gesundheitsschutz 2008; 51(5):483-490. 
[12] Wetterauer B, Schuster R. Seltene Krankheiten. Bundesgesundheitsblatt - Gesundheitsforschung - Gesundheitsschutz 2008; 51(5):519-528.

[13] Deserno TM, Haak D, Brandenburg V, Deserno V, Classen C, Specht P. Integrated Image Data and Medical Record Management for Rare Disease Registries. A General Framework and its Instantiation to the German Calciphylaxis Registry. Journal of Digital Imaging 2014; 27(6):702-713.

[14] Schwanke J, Buckow K, Demiroglu SY, Gusky L, Helbing K, Quade M et al. Eignung eines Customer-Relationship-Management-Systems als Probandenmanagementsystem für klinische Studien. 57. Jahrestagung der Deutschen Gesellschaft für Medizinische Informatik, Biometrie und Epidemiologie e.V. 2012.

[15] McDonald AM, Knight RC, Campbell MK, Entwistle VA, Grant AM, Cook JA et al. What Influences Recruitment to Randomised Controlled Rrials? A Review of Trials Funded by two UK Funding Agencies. Trials 2006; 7:9-16.

[16] Prokosch H, Ganslandt T. Perspectives for Medical Informatics. Methods of Information in Medicine 2009; 48(1):38-44.

[17] De Moor G, Sundgren M, Kalra D, Schmidt A, Dugas M, Claerhout B et al. Using Electronic Health Records for Clinical Research: The Case of the EHR4CR Project. Journal of Biomedical Informatics 2015; 53:162-173.

[18] Soto-Rey I, Trinczek B, Girardeau Y, Zapletal E, Ammour N, Doods J et al. Efficiency and Effectiveness Evaluation of an Automated Multi-Country Patient Count Cohort System. BMC Medical Research Methodology 2015; 15(1):44-53.

[19] Gökbuget N, Hoelzer D. Bedeutung von Multicenterstudiengruppen für die klinische Forschung in der Hämatologie und Onkologie. Bundesgesundheitsblatt - Gesundheitsforschung - Gesundheitsschutz 2009; 52(4):417-424.

[20] Gößwald A, Lange M, Dölle R, Hölling H. Die erste Welle der Studie zur Gesundheit Erwachsener in Deutschland (DEGS1): Gewinnung von Studienteilnehmenden, Durchführung der Feldarbeit und Qualitätsmanagement. Bundesgesundheitsblatt - Gesundheitsforschung - Gesundheitsschutz 2013; 56(5-6):611-619.

[21] Bundesministerium für Bildung und Forschung. Von der Forschung in die Versorgung: Kompetenznetze in der Medizin. Bonn, Berlin: Referat Öffentlichkeitsarbeit; 2006. http://www.gesundheitsforschung-bmbf.de/_media/Kompetenznetze_Forschung_Versorgung.pdf (11.10.2015).

[22] Bundesministerium für Bildung und Forschung. 10 Jahre Kompetenznetze in der Medizin: Kompetenznetze - echte Teamplayer in der Medizin. Bonn, Berlin: Referat Gesundheitsforschung; 2009. http://www.gesundheitsforschung-bmbf.de/_media/BMBF_KompNetz_barrierefrei.pdf (08.06.2014). 
[23] Laan AL van der, Boenink M. Beyond Bench and Bedside: Disentangling the Concept of Translational Research. Health Care Analysis 2015; 23(1):23-49.

[24] Marincola FM. Translational Medicine: A Two-Way Road. Journal of Translational Medicine 2003; 1(1):1.

[25] Rustgi AK. Translational Research: What is it? Gastroenterology 1999; 116(6):1285.

[26] Sonntag K. Implementations of Translational Medicine. Journal of Translational Medicine 2005; 3(1):33-35.

[27] Winter A, Funkat G, Haeber A, Mauz-Koerholz C, Pommerening K, Smers S et al. Integrated Information Systems for Translational Medicine. Methods of Information in Medicine 2007; 46(5):601-607.

[28] Woolf SH. The Meaning of Translational Research and Why It Matters. Journal of American Medical Association 2008; 299(2):211-213.

[29] Bauer U, Niggemeyer E, Lange PE. Das Kompetenznetz Angeborene Herzfehler (KN AHF). Medizinische Klinik 2006; 101(9):753-758.

[30] Leute A, Kirchhof P, Breithardt G, Goette A, Lewalter T, Meinertz T et al. Das Kompetenznetz Vorhofflimmern (AFNET). Medizinische Klinik 2006; 101(8):662-666.

[31] Osterziel KJ, Hewer a. Vernetzte Spitzenforschung in der Kardiologie - das Kompetenznetz Herzinsuffizienz. Zeitschrift für Kardiologie 2005; 94(8):542-544.

[32] Pfeiffer-Gerschel T, Schlee A, Hegerl U. Das Kompetenznetz "Depression, Suizidalität". Medizinische Klinik 2006; 101(6):505-508.

[33] Saußele S, Berger U, Aul C, Büchner T, Döhner H, Ehninger G et al. Klinische Forschung im Kompetenznetz „Akute und chronische Leukämien“. Medizinische Klinik 2006; 101(5):414-420.

[34] Sina C, Schreiber S, Hoffmann JC, Rogler G, Schölmerich J, Zeitz M et al. Das Kompetenznetz chronisch entzündliche Darmerkrankungen (KN-CED)—Vernetzte Forschung führt zur Identifikation von Krankheitsursachen und zur Verbesserung der Patientenversorgung. Medizinische Klinik 2006; 101(2):161-165.

[35] Nolte CH, Villringer A. Kompetenznetz Schlaganfall: Forschen - Fördern - Vernetzen. Medizinische Klinik 2006; 101(3):250-254.

[36] Dr. Thielbeer Consulting. Gutachten zur Evaluation des Förderinstruments »Kompetenznetze in der Medizin«: Kurzfassung im Auftrag des Bundesministeriums für Bildung und Forschung (BMBF). Hambug; 2012. http://www.gesundheitsforschungbmbf.de/_media/Evaluation_Kompetenznetze_Kurzfassung.pdf (11.10.2015).

[37] Bundesministerium für Bildung und Forschung. Rahmenprogramm Gesundheitsforschung der Bundesregierung. Bonn, Berlin: Referat Gesundheitsforschung; 2010. 
http://www.gesundheitsforschung-bmbf.de/_media/Gesundheitsforschungsprogramm.pdf (11.10.2015).

[38] Ohmann C, Kuchinke W. Future Developments of Medical Informatics from the Viewpoint of Networked Clinical Research. Methods of Information in Medicine 2009; 48(1):45-54.

[39] Stausberg J, Altmann U, Antony G, Drepper J, Sax U, Schütt A. Registers for Networked Medical Research in Germany. Applied Clinical Informatics 2010; 1(4):408418.

[40] Wichmann H, Kaaks R, Hoffmann W, Jöckel K, Greiser KH, Linseisen J. Die Nationale Kohorte. Bundesgesundheitsblatt - Gesundheitsforschung - Gesundheitsschutz 2012; 55(6-7):781-789.

[41] Helbing K, Demiroglu SY, Rakebrandt F, Pommerening K, Rienhoff O, Sax U. A Data Protection Scheme for Medical Research Networks. Methods of Information in Medicine 2010; 49(6):601-607.

[42] Pommerening K. Pseudonyme - ein Kompromiß zwischen Anonymisierung und Personenbezug. 40. Jahrestagung der Deutschen Gesellschaft für Medizinische Informatik, Biometrie und Epidemiologie e.V. 1995.

[43] Pommerening K, Wagner M. Ein Pseudonymisierungdienst für medizinische Forschungsnetze. Informatik, Biometrie und Epidemiologie in Medizin und Biologie 2001.

[44] Aamot H, Kohl CD, Richter D, Knaup-Gregori P. Pseudonymization of Patient Identifiers for Translational Research. BMC Medical Informatics and Decision Making 2013; 13(1):75-89.

[45] Bialke M, Langner D, Geidel L, Bahls T, Havemann C, Piegsa J et al. "Who am I? And if so, how many?” - The E-PIX as Innovative System to Manage Person Identities. 2nd Research Data Management Workshop 2014.

[46] Reng C, Pommerening K, Specker C, Debold P. Generische Lösungen der TMF zum Datenschutz für die Forschungsnetze in der Medizin: Im Auftrag des Koordinierungsrates der Telematikplattform für Medizinische Forschungsnetze. 1. Auflage. Berlin: MWV Medizinisch Wissenschaftliche Verlagsgesellschaft; 2006.

[47] Pommerening K, Drepper J, Helbing K, Ganslandt T. Leitfaden zum Datenschutz in medizinischen Forschungsprojekten: Generische Lösungen der TMF 2.0. 1. Auflage. Berlin: MWV Medizinisch Wissenschaftliche Verlagsgesellschaft; 2014.

[48] Schwanke J, Löhnhardt B. TMF Workshop Probandenverwaltung. http://www.tmfev.de/News/articleType/ArticleView/articleId/1334.aspx (11.10.2015). 
[49] Grätzel P von Grätz. Die Deutschen Zentren der Gesundheitsforschung: Gebündelte Erforschung von Volkskrankheiten. Bonn, Berlin: Referat Lebenswissenschaftliche Forschungseinrichtungen; 2011. http://www.gesundheitsforschung-bmbf.de/_media/DZG_barrierefrei.pdf (11.10.2015).

[50] Borg A, Lablans M. Clinical Communication Platform (CCP-IT): Datenschutzkonzept im Deutschen Konsortium für Translationale Krebsforschung (DKTK): DKTK; 2014. http://www.unimedizin-mainz.de/imbei/informatik/projekte/aktuell/dktk.html (11.10.2015).

[51] DZNE Datenschutzkonzept: Datenbanken der Klinischen Forschung; 2014.

[52] Verfahrensbeschreibung und Datenschutzkonzept des Zentralen Datenmanagements des DZHK; 2014.

[53] Majeed RW, Kuhn S, Ruppert C, Günther A, Röhrig R. Zentrale Forschungsdatenbank im dezentralen Forschungsnetz - Datenschutzkonzept des Deutschen Zentrums für Lungenforschung (DZL). 59. Jahrestagung der Deutschen Gesellschaft für Medizinische Informatik, Biometrie und Epidemiologie e.V. 2014.

[54] Neubauer T, Heurix J. A Methodology for the Pseudonymization of Medical Data. International Journal of Medical Informatics 2011; 80(3):190-204.

[55] Somolinos R, Munoz A, Hernando ME, Pascual M, Caceres J, Sanchez-de-Madariaga R et al. Service for the Pseudonymization of Electronic Healthcare Records based on ISO/EN 13606 for the Secondary Use of Information. IEEE Journal of Biomedical and Health Informatics 2014; [in Druck].

[56] Bredfeldt CE, Butani A, Padmanabhan S, Hitz P, Pardee R. Managing Protected Health Information in Distributed Research Network Environments: Automated Review to Facilitate Collaboration. BMC Medical Informatics and Decision Making 2013; 13(1):3945 .

[57] Lusignan S de. Effective Pseudonymisation and Explicit Statements of Public Interest to Ensure the Benefits of Sharing Health Data for Research, Quality Improvement and Health Service Management Outweigh the Risks. Informatics in Primary Care 2014; 21(2):61-63.

[58] Mehta SR, Vinterbo SA, Little SJ. Ensuring Privacy in the Study of Pathogen Genetics. The Lancet Infectious Diseases 2014; 14(8):773-777.

[59] Pommerening K. Das Datenschutzkonzept der TMF für Biomaterialbanken: The TMF Data Protection Scheme for Biobanks. Information Technology 2007; 49(6):352-359.

[60] Prokosch H, Beck A, Ganslandt T, Hummel M, Kiehntopf M, Sax U et al. IT Infrastructure Components for Biobanking. Applied Clinical Informatics 2010; 1(4):419-429. 
[61] Grabe HJ, Assel H, Bahls T, Dörr M, Endlich K, Endlich N et al. Cohort Profile: Greifswald Approach to Individualized Medicine (GANI_MED). Journal of Translational Medicine 2014; 12(1):144-158.

[62] McQueen MJ, Keys JL, Bamford K, Hall K. The Challenge of Establishing, Growing and Sustaining a Large Biobank: A Personal Perspective. Clinical Biochemistry 2014; 47(4-5):239-244.

[63] Waltz E. Pricey Cancer Genome Project Struggles with Sample Shortage. Nature Medicine 2007; 13(4):391.

[64] Ransohoff DF, Gourlay ML. Sources of Bias in Specimens for Research About Molecular Markers for Cancer. Journal of Clinical Oncology 2010; 28(4):698-704.

[65] Reed W, Jor S, Bjugn R. How can Clinical Biobanks and Patient Information be Adapted for Research - Establishing a Hospital Based Data Warehouse Solution. Norwegian Journal of Epidemiology 2012; 21(2):191-194.

[66] Imboden M, Probst-Hensch NM. Biobanking Across the Phenome - At the Center of Chronic Disease Research. BMC Public Health 2013; 13(1):1094-1106.

[67] Krawczak M, Semler SC, Kiehntopf M. Biobanken. Medizinische Genetik 2010; 22(2):229-234.

[68] Simeon-Dubach D, Watson P. Biobanking 3.0: Evidence Based and Customer Focused Biobanking. Clinical Biochemistry 2014; 47(4-5):300-308.

[69] Berlit P. Klinische Neurologie. 3. Auflage. Berlin: Springer; 2011.

[70] Buckow K, Quade M, Rienhoff O, Nussbeck SY. Changing Requirements and Resulting Needs for IT-infrastructure for Longitudinal Research in the Neurosciences. Neuroscience Research 2014; [in Druck].

[71] Winter A, Haux R, Ammenwerth E, Brigl B, Hellrung N, Jahn F. Health Information Systems: Architectures and Strategies. 2. Auflage. London: Springer; 2011.

[72] Köpcke F, Trinczek B, Majeed RW, Schreiweis B, Wenk J, Leusch T et al. Evaluation of Data Completeness in the Electronic Health Record for the Purpose of Patient Recruitment into Clinical Trials: A Retrospective Analysis of Element Presence. BMC Medical Informatics and Decision Making 2013; 13(1):37-44.

[73] Bower P, Wallace P, Ward E, Graffy J, Miller J, Delaney B et al. Improving Recruitment to Health Research in Primary Care. Family Practice 2009; 26(5):391-397.

[74] Doods J, Bache R, McGilchrist M, Daniel C, Dugas M, Fritz F. Piloting the EHR4CR Feasibility Platform Across Europe. Methods of Information in Medicine 2014; 53(4):264-268. 
[75] Trinczek B, Köpcke F, Leusch T, Majeed RW, Schreiweis B, Wenk J et al. Design and Multicentric Implementation of a Generic Software Architecture for Patient Recruitment Systems Re-Using Existing HIS Tools and Routine Patient Data. Applied Clinical Informatics 2014; 5(1):264-283.

[76] Schreiweis B, Trinczek B, Köpcke F, Leusch T, Majeed RW, Wenk J et al. Comparison of Electronic Health Record System Functionalities to Support the Patient Recruitment Process in Clinical Trials. International Journal of Medical Informatics 2014; 83(11):860-868.

[77] Helbing K. Erweiterung des Konzeptes einer Patientenakte nach § 291a SGB V um eine Schnittstelle für die medizinische Forschung. Göttingen: Georg-August-Universität Göttingen; 2013.

[78] Coorevits P, Sundgren M, Klein GO, Bahr A, Claerhout B, Daniel C et al. Electronic Health Records: New Opportunities for Clinical Research. Journal of Internal Medicine 2013; 274(6):547-560.

[79] Yen J, Chiu W, Chu S, Hsu M. Secondary Use of Health Data. Journal of the Formosan Medical Association 2015 [in Druck].

[80] Leung KYE, van der Lijn F, Vrooman HA, Sturkenboom, Miriam C J M, Niessen WJ. IT Infrastructure to Support the Secondary Use of Routinely Acquired Clinical Imaging Data for Research. Neuroinformatics 2015; 13(1):65-81.

[81] Pommerening K, Reng M. Secondary Use of the EHR via Pseudonymisation. Studies in Health Technology and Informatics 2004; 103(1):441-446.

[82] Kushida CA, Nichols DA, Jadrnicek R, Miller R, Walsh JK, Griffin K. Strategies for De-Identification and Anonymization of Electronic Health Record Data for Use in Multicenter Research Studies. Medical Care 2012; 50(1):82-101.

[83] Riou C, Fresson J, Serre JL, Avillach P, Leneveut L, Quantin C. Guide to Good Practices to Ensure Privacy Protection in Secondary Use of Medical Records. Revue d'Épidémiologie et de Santé Publique 2014; 62(3):207-214.

[84] Fenz S, Heurix J, Neubauer T, Rella A. De-Identification of Unstructured Paper-Based Health Records for Privacy-Preserving Secondary Use. Journal of Medical Engineering \& Technology 2014; 38(5):260-268.

[85] Nitzlnader M, Schreier G. Patient Identity Management for Secondary Use of Biomedical Research Data in a Distributed Computing Environment. Studies in Health Technology and Informatics 2014; 198(1):211-218.

[86] Lablans M, Borg A, Ückert F. A RESTful Interface to Pseudonymization Services in Modern Web Applications. BMC Medical Informatics and Decision Making 2015; 15(1):2-11. 
[87] Schnell R, Bachteler T, Reiher J. Privacy-Preserving Record Linkage using Bloom Filters. BMC Medical Informatics and Decision Making 2009; 9(1):41-51.

[88] Cramon P, Rasmussen AK, Bonnema SJ, Bjorner JB, Feldt-Rasmussen U, Groenvold M et al. Development and Implementation of PROgmatic: A Clinical Trial Management System for Pragmatic Multi-Centre Trials, Optimised for Electronic Data Capture and Patient-Reported Outcomes. Clinical Trials 2014; 11(3):344-354.

[89] Durkalski V, Wenle Zhao, Dillon C, Kim J. A Web-Based Clinical Trial Management System for a Sham-Controlled Multicenter Clinical Trial in Depression. Clinical Trials 2010; 7(2):174-182.

[90] Fraser HSF, Thomas D, Tomaylla J, Garcia N, Lecca L, Murray M et al. Adaptation of a Web-Based, Open Source Electronic Medical Record System Platform to Support a Large Study of Tuberculosis Epidemiology. BMC Medical Informatics and Decision Making 2012; 12(1):125-134.

[91] Nesbitt G, McKenna K, Mays V, Carpenter A, Miller K, Williams M. The Epilepsy Phenome/Genome Project (EPGP) Informatics Platform. International Journal of Medical Informatics 2013; 82(4):248-259.

[92] BRISSKIT. Biomedical Research Infrastructure Software Service Kit: Towards a Cloud-Enabled Translational Research IT Platform. Leicester; 2012.

[93] CaBIG. CaBIG ${ }^{\circledR}$ Central Clinical Participant Registry (C3PR): Study participant registry. Bethesda: U.S. Dept. of Health and Human Services; 2010.

[94] Nelson EK, Piehler B, Rauch A, Ramsay S, Holman D, Asare S et al. Ancillary Study Management Systems: A Review of Needs. BMC Medical Informatics and Decision Making 2013; 13(1):5-20.

[95] Geyer J, Myers K, Vander Stoep A, McCarty C, Palmer N, DeSalvo A. Implementing a Low-Cost Web-Based Clinical Trial Management System for Community Studies: A Case Study. Clinical Trials 2011; 8(5):634-644.

[96] Payne, Philip R O, Embi PJ, Johnson SB, Mendonca E, Starren J. Improving Clinical Trial Participant Tracking Tools Using Knowledge-Anchored Design Methodologies. Applied Clinical Informatics 2010; 1(2):177-196.

[97] Kaur M, Sprague S, Ignacy T, Thoma A, Bhandari M, Farrokhyar F. How to Optimize Participant Retention and Complete Follow-up in Surgical Research. Canadian Journal of Surgery 2014; 57(6):420-427.

[98] Ahrens W, Greiser H, Linseisen J, Kluttig A, Schipf S, Schmidt B et al. Das Design der Machbarkeitsstudien für eine bundesweite Kohortenstudie in Deutschland: Die Pretests der Nationalen Kohorte (NAKO). Bundesgesundheitsblatt, Gesundheitsforschung, Gesundheitsschutz 2014; 57(11):1246-1254. 
[99] Pohl K. Requirements Engineering: Grundlagen, Prinzipien, Techniken. 2. korrigierte Auflage. Heidelberg: dpunkt.verlag; 2008.

[100] Rolland C, Grosz G, Kla R. Experience With Goal-Scenario Coupling in Requirements Engineering. 4th IEEE International Symposium on Requirements Engineering 1999.

[101] Misra S, Kumar V, Kumar U. Goal-oriented or Scenario-based Requirements Engineering Technique - What Should a Practitioner Select? Canadian Conference on Electrical and Computer Engineering 2005.

[102] Lamsweerde A van, Willemet L. Inferring Declarative Requirements Specifications From Operational Scenarios. IEEE Transactions on Software Engineering 1998; 24(12):1089-1114.

[103] Lamsweerde A van. Requirements Engineering: From System Goals to UML Models to Software Specifications. Chichester, Hoboken: John Wiley; 2009.

[104] Lamsweerde A van. Goal-Oriented Requirements Engineering: A Guided Tour. Fifth IEEE International Symposium on Requirements Engineering 2001.

[105] Lamsweerde A van. Goal-Oriented Requirements Enginering: A Roundtrip from Research to Practice. 12th IEEE International Requirements Engineering Conference 2004.

[106] Pohl K, Rupp C. Basiswissen Requirements Engineering: Aus- und Weiterbildung zum "Certified Professional for Requirements Engineering"; Foundation Level nach IREBStandard. 3. korrigierte Auflage. Heidelberg: dpunkt.verlag; 2011.

[107] Scholl A. Die Befragung. 3. überarbeitete Auflage. Konstanz: UVK Verlagsgesellschaft $\mathrm{mbH} ; 2014$.

[108] Rupp C, die SOPHISTen. Requirements-Engineering und -Management: Professionelle, iterative Anforderungsanalyse für die Praxis. 5. aktualisierte und erweiterte Auflage. München: Hanser; 2009.

[109] Schwanke J, Nussbeck SY, Helbing K, Rienhoff O. Entwicklung eines Probandenmanagementsystems für ein multizentrisches Forschungsprojekt. 58. Jahrestagung der Deutschen Gesellschaft für Medizinische Informatik, Biometrie und Epidemiologie e.V. 2013.

[110] Mayring P. Qualitative Inhaltsanalyse: Grundlagen und Techniken. 11. aktualisierte und überarbeitete Auflage. Weinheim: Beltz; 2010.

[111] Diekmann A. Empirische Sozialforschung: Grundlagen, Methoden, Anwendungen. 6. Auflage. Reinbek: Rowohlt; 2012.

[112] Meuser M, Nagel U. ExpertInneninterviews - vielfach erprobt, wenig bedacht: ein Beitrag zur qualitativen Methodendiskussion. In: Garz D, Kraimer K. Qualitativ-empirische Sozialforschung: Konzepte, Methoden, Analysen. Opladen: Westdeutscher Verlag; 1991. S. 441-71. 
[113] Rupp C, Queins S, die SOPHISTen. UML 2 glasklar: Praxiswissen für die UML-Modellierung. 4. aktualisierte und erweiterte Auflage. München: Hanser; 2012.

[114] Sommerville I. Software Engineering. 9. aktualisierte Auflage. München: Pearson Studium; 2012.

[115] Stahmann A, Bauer CRKD, Schwanke J. Concept for Linking De-Identified Biomedical Research Data Using a Study Participant Management System. Studies in Health Technology and Informatics 2014; 205(1):848-852.

[116] Stahmann A, Bauer CRKD, Schulze TG, Schwanke J. Standortbezogene Datenzusammenführung in Verbundforschungsvorhaben durch ein Probandenmanagementsystem. 59. Jahrestagung der Deutschen Gesellschaft für Medizinische Informatik, Biometrie und Epidemiologie e.V. 2014.

[117] Schwarzmaier M. Erweiterung eines Probandenmanagementsystems zur Generierung von übergreifenden Forschungspseudoynmen [Bachelorarbeit]. Göttingen: Georg-August-Universität Göttingen; 2014.

[118] Matoni M. Abbildung und Verwaltung von Einwilligungserklärungen in einem Probandenmanagementsystem [Masterarbeit]. Göttingen: Georg-August-Universität Göttingen; 2014.

[119] Gharbi M, Koschel A, Rausch A, Starke G. Basiswissen für Software-Architekten: Ausund Weiterbildung zum Certified Professional for Software Architecture - Foundation Level nach iSAQB-Standard. 1. Auflage. Heidelberg: dpunkt.verlag; 2012.

[120] Starke G. Effektive Softwarearchitekturen: Ein praktischer Leitfaden. 6., überarbeite Auflage. München: Hanser; 2014.

[121] Evans E. Domain-Driven Design: Tackling Complexity in the Heart of Softwares. Boston: Addison-Wesley; 2004.

[122] Vernon V. Implementing Domain-Driven Design. Upper Saddle River: Addison-Wesley; 2012.

[123] Cockburn A. Hexagonal Architecture; 2008 (11.10.2014). http://alistair.cockburn.us/Hexagonal+architecture.

[124] Freeman S, Pryce N. Growing Object-Oriented Software, Guided by Tests. Upper Saddle River: Addison-Wesley; 2010.

[125] Gamma E, Helm R, Johnson R, Vlissides J. Entwurfsmuster: Elemente wiederverwendbarer objektorientierter Software. 1. Auflage. München: Addison-Wesley; 2004.

[126] Fowler M. Inversion of Control Containers and the Dependency Injection pattern. http://martinfowler.com/articles/injection.html (11.10.2015).

[127] Fowler M, Rice D, Foemmel M, Hieatt E, Mee R, Stattford R. Patterns of Enterprise Application Architecture. Boston: Addison-Wesley; 2003. 
[128] Ludewig J, Lichter H. Software Engineering: Grundlagen, Menschen, Prozesse, Techniken. 3. Auflage. Heidelberg: dpunkt.verlag; 2013.

[129] Oracle Corporation. Java Platform, Standard Edition 8 API Specification; 2015 (11.10.2015). https://docs.oracle.com/javase/8/docs/api/.

[130] Murphy SN, Weber G, Mendis M, Gainer V, Chueh HC, Churchill S et al. Serving the Enterprise and Beyond with Informatics for Integrating Biology and the Bedside (i2b2). Journal of the American Medical Informatics Association 2010; 17(2):124-130.

[131] Marcus DS, Olsen TR, Ramaratnam M, Buckner RL. The Extensible Neuroimaging Archive Toolkit: An Informatics Platform for Managing, Exploring, and Sharing Neuroimaging Data. Neuroinformatics 2007; 5(1):11-34.

[132] Mamlin BW, Biondich PG, Wolfe BA, Fraser H, Jazayeri D, Allen C et al. Cooking up an Open Source EMR for Developing Countries: OpenMRS - A Recipe for Successful Collaboration. AMIA Annual Symposium Proceedings 2006.

[133] Dowst H, Pew B, Watkins C, McOwiti A, Barney J, Qu S et al. Acquire: An OpenSource Comprehensive Cancer Biobanking System. Bioinformatics 2015; 31(10):16551662.

[134] Bauch A, Adamczyk I, Buczek P, Elmer F, Enimanev K, Glyzewski P et al. openBIS: A Flexible Framework for Managing and Analyzing Complex Data in Biology Research. BMC Bioinformatics 2011; 12(1):468-486.

[135] Wikipedia. Java-Bibliotheken. https://de.wikipedia.org/w/index.php?title=Kategorie:Java-Bibliothek\&oldid=116844170 (11.10.2015).

[136] Humble J, Farley D. Continuous Delivery. Upper Saddle River: Addison-Wesley; 2011.

[137] Schaefer C, Ho C, Harrop R. Pro Spring. 4. Auflage. New York: Apress; 2014.

[138] Walls C. Spring in Action. 4. Auflage. Shelter Island: Manning; 2014.

[139] Saake G, Heuer A, Sattler K. Datenbanken: Konzepte und Sprachen. 2. Auflage. Heidelberg: Mitp; 2007.

[140] Müller B, Wehr H. Java Persistence API 2: Hibernate, EclipseLink, OpenJPA und Erweiterungen. München: Hanser; 2012.

[141] Baumann J, Arndt D, Engelen F, Hardy F, Mjartan C. Vaadin: Der kompakte Einstieg für Java-Entwickler. 1. Auflage. Heidelberg: dpunkt.verlag; 2014.

[142] Vaadin Ltd. Official Vaadin Spring Support. https://vaadin.com/spring (11.10.2015).

[143] Boehm BW. Verifying and Validating Software Requirements and Design Specifications. IEEE Software 1984; 1(1):75-88.

[144] Barnum CM. Usability Testing Essentials. Amsterdam: Morgan Kaufmann Publishers; 2011. 
[145] Brooke J. SUS - A Quick and Dirty Usability Scale. Usability Evaluation in Industry 1996; 189(194):4-7.

[146] Rummel B. System Usability Scale - Jetzt auch auf Deutsch. https://experience.sap.com/skillup/system-usability-scale-jetzt-auch-auf-deutsch/ (11.10.2015).

[147] Alroobaea R, Mayhew PJ. How Many Participants are Really Enough for Usability Studies? Science and Information Conference 2014.

[148] Faulkner L. Beyond the Five-User Assumption: Benefits of Increased Sample Sizes in Usability Testing. Behavior Research Methods, Instruments, \& Computers 2003; 35(3):379-383.

[149] Macefield R, Close C, Nu WW. How To Specify the Participant Group Size for Usability Studies: A Practitioner's Guide. Journal of Usability Studies 2009; 5(1):34-45.

[150] Weiß C. Basiswissen Medizinische Statistik. 4. überarbeite Auflage. Heidelberg: Springer; 2008.

[151] Bangor A, Kortum PT, Miller JT. An Empirical Evaluation of the System Usability Scale. International Journal of Human-Computer Interaction 2008; 24(6):574-594.

[152] Pommerening K, Miller M, Schmidtmann I, Michaelis J. Pseudonyms for Cancer Registries. Methods of Information in Medicine 1996; 35(2):112-121.

[153] Arab L, Hahn H, Henry J, Chacko S, Winter A, Cambou MC. Using the Web for Recruitment, Screen, Tracking, Data Management, and Quality Control in a Dietary Assessment Clinical Validation Trial. Contemporary Clinical Trials 2010; 31(2):138-146.

[154] Heinze O, Birkle M, Köster L, Bergh B. Architecture of a Consent Management Suite and Integration into IHE-based Regional Health Information Networks. BMC Medical Informatics and Decision Making 2011; 11(1):58-68.

[155] Lehmann TM. Handbuch der Medizinischen Informatik. 2. vollständig neu bearbeitete Auflage. München: Hanser; 2005.

[156] ISPE. GAMP5: Ein risikobasierter Ansatz für konforme GxP-computergestützte Systeme; 2008.

[157] Concept Heidelberg. GMP-/FDA-gerechte Validierung: Qualifizierung von Anlagen und Validierung von Prozessen und Systemen. 1. Auflage. Aulendorf: Editio Cantor Verlag; 2010.

[158] Zörner S. LDAP für Java-Entwickler: Einstieg und Integration. 4 Auflage: entwickler press; 2013.

[159] Vaadin Ltd. Vaadin Framework Features. https://vaadin.com/features (11.10.2015).

[160] Deutsches Register Klinischer Studien. http://www.germanctr.de (11.10.2015).

[161] Bundesministerium für Gesundheit. Gesetzentwurf der Bundesregierung: Entwurf eines Gesetzes für sichere digitale Kommunikation und Anwendungen im Gesundheitswesen 
(E-Health-Gesetz). http://www.bmg.bund.de/fileadmin/dateien/Downloads/E/eHealth/150527_Gesetzentwurf_E-Health.pdf (11.11.2015).

[162] Pressemitteilung: "Digitale Vernetzung stärkt die Patienten" E-Health Gesetzentwurf in erster Lesung im Deutschen Bundestag. http://www.bmg.bund.de/fileadmin/dateien/Pressemitteilungen/2015/2015_03/150703-25_PM_1_Lesung_E-Health.pdf (11.10.2015). 


\section{Abbildungsverzeichnis}

Abbildung 1. Minimalbeispiel eines Aktivitätsdiagramms ................................................ 11

Abbildung 2. Architekturstil „Ports and Adapters“ [120] ............................................... 16

Abbildung 3. Minimalbeispiel eines Klassendiagramms zur Modellierung des

Domänenmodells

Abbildung 4. Minimalbeispiel eines Klassendiagramms zur Modellierung der primären und sekundären Schnittstellen

Abbildung 5. Minimalbeispiel eines Komponentendiagramms zur Beschreibung des Aufbaus und der Struktur des Prototyps ......................................................... 19

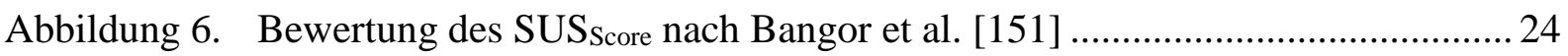

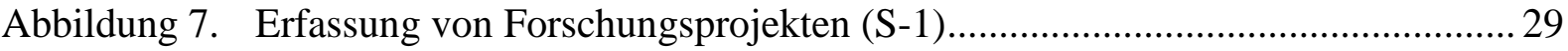

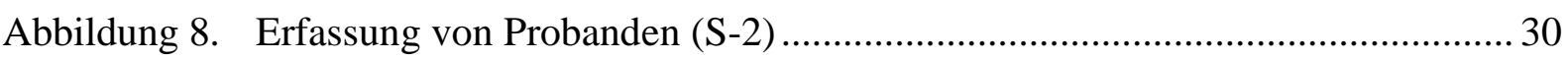

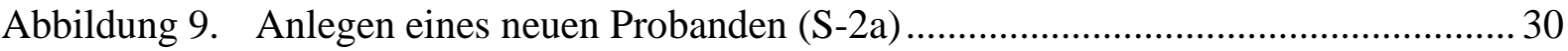

Abbildung 10. Änderung von Probandenstammdaten (S-3) ................................................... 31

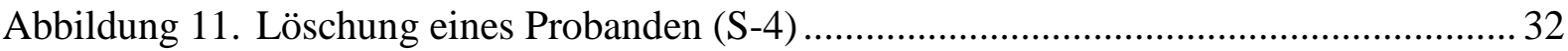

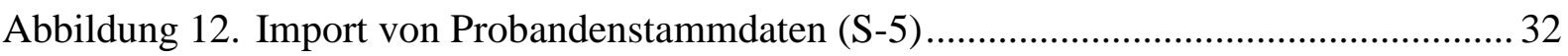

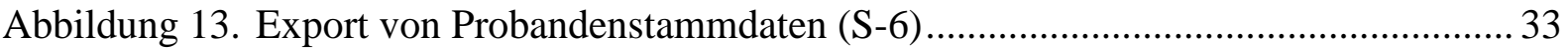

Abbildung 14. Abbildung der Einwilligungserklärung (S-7) ............................................... 33

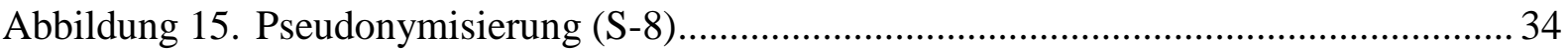

Abbildung 16. Generierung von projektübergreifenden Forschungspseudonymen (S-9) ....... 35

Abbildung 17. Domänenmodell des Bereichs Systemkonfiguration....................................... 44

Abbildung 18. Domänenmodell des Bereichs Sicherheit ..................................................... 45

Abbildung 19. Domänenmodell des Bereichs Projektverwaltung......................................... 46

Abbildung 20. Domänenmodell des Bereichs Probandenverwaltung .................................... 46

Abbildung 21. Domänenmodell des Bereichs Forschungsakte ............................................... 47

Abbildung 22. Domänenmodell des Bereichs Kommunikation ............................................ 48

Abbildung 23. Domänenmodell des Bereichs Pseudonymisierung....................................... 49

Abbildung 24. RecordLinkageProvider des Bereichs Probandenverwaltung ......................... 50

Abbildung 25. HISProvider des Bereichs Probandenverwaltung............................................ 51

Abbildung 26. ExternalSystemProvider des Bereichs Probandenverwaltung......................... 52

Abbildung 27. IdentifierGeneratorProvider des Bereichs Pseudonymisierung........................ 52

Abbildung 28. Struktur und Aufbau des Probandenmanagementsystems................................ 54

Abbildung 29. Änderungsnachverfolgung auf Datenbankebene ........................................... 55

Abbildung 30. Loginseite des Probandenmanagementsystems ............................................. 56

Abbildung 31. Probandenübersicht des Probandenmanagementsystems ................................ 57

Abbildung 32. Detailansicht eines Probanden im Probandenmanagementsystem .................. 57

Abbildung 33. Forschungsaktenansicht eines Projekts im Probandenmanagementsystem..... 58

Abbildung 34. Forschungsakte eines Probanden im Probandenmanagementsystem .............. 59

Abbildung 35. Administrative Detailansicht eines Forschungsprojekts im

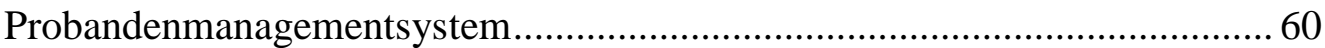

Abbildung 36. Benutzerverwaltung im Probandenmanagementsystem ................................. 60 
Abbildung 37. Login des Probandenmanagementsystems zur Benutzerauthentifizierung ..... 61

Abbildung 38. Systemkonfiguration zur Einstellung eines zentralen

Benutzerverzeichnisdiensts.

Abbildung 39. Auswahl des Authentifizierungsverfahrens beim Anlegen eines neuen

Benutzers

Abbildung 40. Einstellungen für einen lokalen bzw. zentralen Benutzer ............................... 62

Abbildung 41. Projektverwaltung im Administrationsbereich ............................................ 63

Abbildung 42. Anlegen, Bearbeiten und Löschen von Forschungsprojekten ......................... 63

Abbildung 43. Verfügbare Reiter zur Erfassung ergänzender Daten für ein

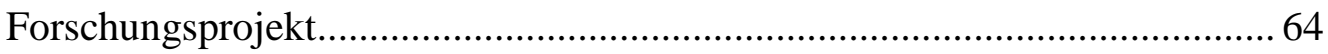

Abbildung 44. Zuordnung von Rechten zu projektspezifischen Rollen ................................. 64

Abbildung 45. Übersicht über die einem Benutzer zugeordneten Forschungsprojekte .......... 65

Abbildung 46. Verwaltung der Probandenstammdaten im Forschungsprojekt ....................... 65

Abbildung 47. Zuordnung von Probanden zu Forschungsprojekten durch die

Forschungsakte 66

Abbildung 48. Übersicht über alle einem Benutzer zugeordneten Probanden....

Abbildung 49. Änderung und Löschung von Probandenstammdaten mit entsprechenden

Rechten

Abbildung 50. Löschen eines Probanden aus Sicht der Datenbank - Schritt 1

Abbildung 51. Löschen eines Probanden aus Sicht der Datenbank - Schritt 2 ....................... 68

Abbildung 52. Löschen eines Probanden aus Sicht der Datenbank - Schritt 3....................... 68

Abbildung 53. Dialog zur Auswahl, wie eine neue Forschungsakte angelegt werden soll ..... 68

Abbildung 54. Anlegen eines neuen Probanden durch Eingabe der Stammdaten................... 69

Abbildung 55. Übernahme eines Probanden aus einem vorhandenen Forschungsprojekt...... 69

Abbildung 56. Abruf der Probandenstammdaten aus dem KIS............................................. 70

Abbildung 57. Erfassung von projektspezifischen Daten für die Forschungsakte .................. 70

Abbildung 58. Beispiel für die Duplikaterkennung im Probandenmanagementsystem -

Schritt 1

Abbildung 59. Beispiel für die Duplikaterkennung im Probandenmanagementsystem Schritt 2

Abbildung 60. Beispieldatensatz für den Import ins Probandenmanagementsystem.............. 72

Abbildung 61. Importierte Beispieldaten im Probandenmanagementsystem........................... 72

Abbildung 62. Export von Stammdaten aus dem Probandenmanagementsystem.................... 72

Abbildung 63. Reiter Pseudonymisierung in der Forschungsakte eines Probanden ............... 73

Abbildung 64. Suche nach Probanden anhand von Pseudonymen .......................................... 73

Abbildung 65. Dialog zur Auswahl, wie ein neues Pseudonym erstellt werden soll .............. 74

Abbildung 66. Dialog zum Abruf des Pseudonyms von einem externen System

(PIDGenerator)

Abbildung 67. Zuordnung des Pseudonyms zu den Probandenstammdaten in der

Datenbank des externen Systems................................................................. 74

Abbildung 68. Dialog zur Dokumentation von Metadaten für ein Pseudonym ...................... 75 
Abbildung 69. Ergänzung des Domänenmodells des Bereichs Probandenverwaltung um Kontaktinformationen zu einem Probanden

Abbildung 70. Primäre und sekundäre Schnittstellen zur Verwaltung der Entities des Bereichs Systemkonfiguration

Abbildung 71. Primäre und sekundäre Schnittstellen zur Verwaltung der Entities des Bereichs Projektverwaltung

Abbildung 72. Primäre und sekundäre Schnittstellen zur Verwaltung der Entities des Bereichs Probandenverwaltung

Abbildung 73. Primäre und sekundäre Schnittstellen zur Verwaltung der Entities des Bereichs Forschungsakte

Abbildung 74. Primäre und sekundäre Schnittstellen zur Verwaltung der Entities des Bereichs Kommunikation ...

Abbildung 75. Primäre und sekundäre Schnittstellen zur Verwaltung der Entities des Bereichs Pseudonymisierung 


\section{Tabellenverzeichnis}

Tabelle 1. Vorlage zur strukturierten Zusammenfassung der Interviews......................... 10

Tabelle 2. Vorlage zur zusammenfassenden Darstellung der identifizierten Ziele .......... 11

Tabelle 3. Vorlage zur zusammenfassenden Darstellung der identifizierten Szenarien .. 12

Tabelle 4. Vorlage zur strukturierten Beschreibung der lösungsorientierten

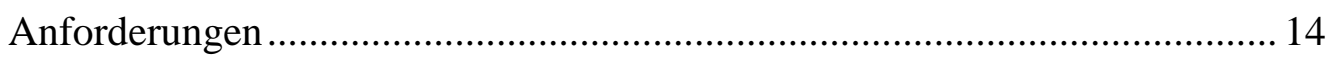

Tabelle 5. Vorlage zur Dokumentation des Erfüllungsgrades der Ziele und Szenarien... 21

Tabelle 6. Vorlage zur Darstellung der Ergebnisse des System Usability Scale.............. 25

Tabelle 7. Zusammenfassung der 16 identifizierten Ziele.............................................26

Tabelle 8. Zusammenfassung der neun identifizierten Szenarien ................................... 28

Tabelle 9. Lösungsorientierte funktionale Anforderungen der Kategorie

Authentifizierung und Autorisierung.............................................................. 36

Tabelle 10. Lösungsorientierte funktionale Anforderungen der Kategorie Verwaltung von Forschungsprojekten............................................................................... 37

Tabelle 11. Lösungsorientierte funktionale Anforderungen der Kategorie Verwaltung

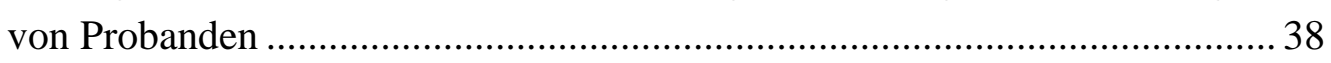

Tabelle 12. Lösungsorientierte funktionale Anforderungen der Kategorie Verwaltung

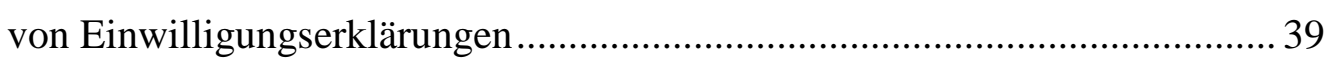

Tabelle 13. Lösungsorientierte funktionale Anforderungen der Kategorie

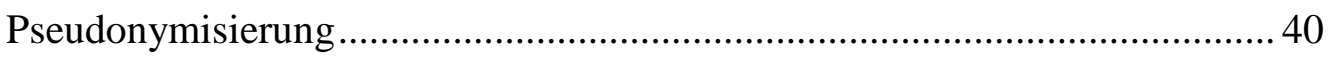

Tabelle 14. Lösungsorientierte Qualitätsanforderungen.................................................. 41

Tabelle 15. Lösungsorientierte Randbedingungen ......................................................... 42

Tabelle 16. Dokumentation des Erfüllungsgrades der Ziele und Szenarien....................... 76

Tabelle 17. Bewertung der Vorversion des Probandenmanagementsystems ..................... 77

Tabelle 18. Bewertung des entwickelten Probandenmanagementsystems......................... 80

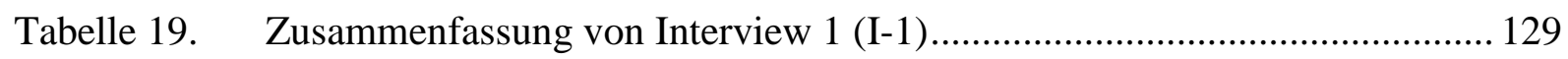

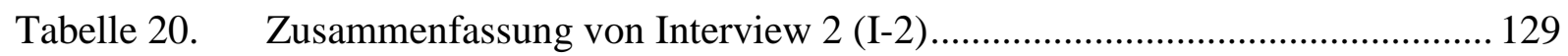

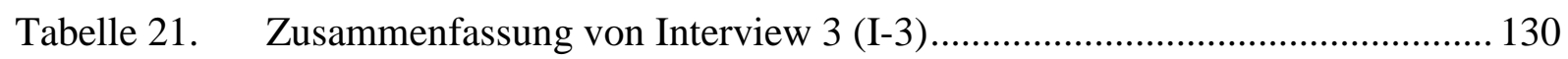

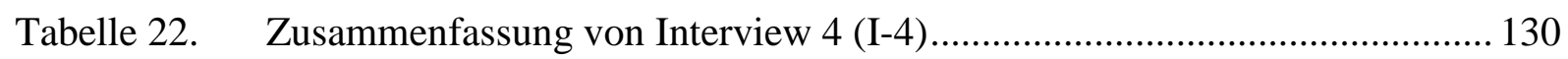

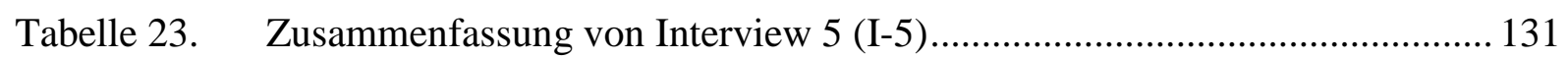

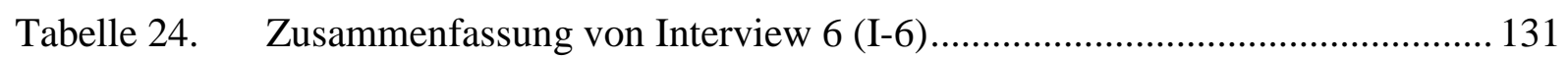

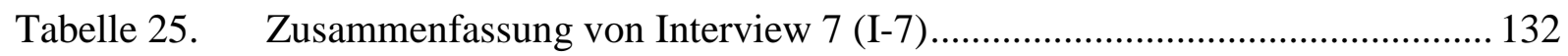

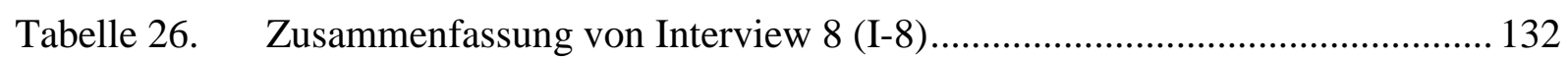

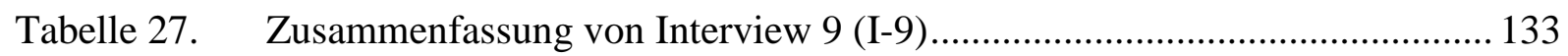

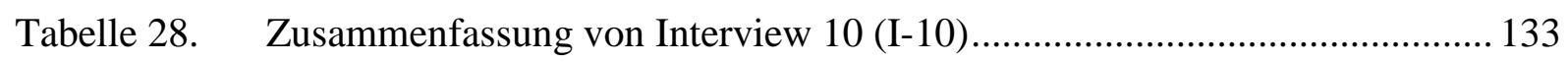

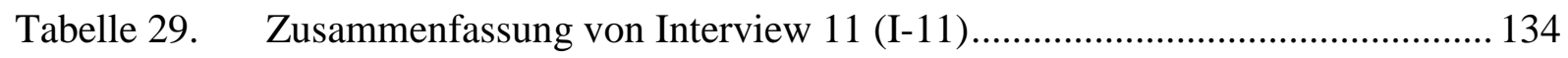

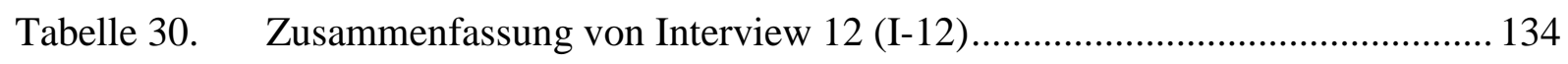

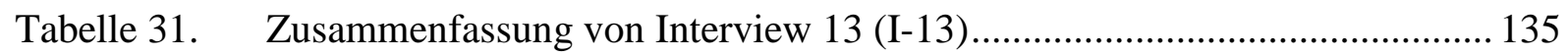

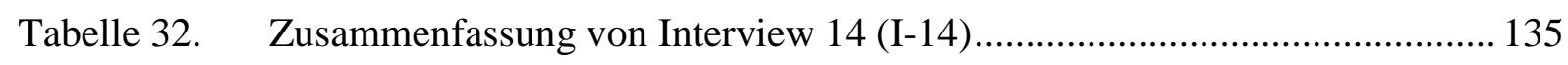

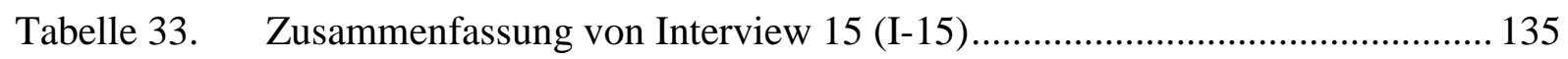

Tabelle 34. Zusammenfassung von Interview 16 (I-16)................................................ 136 
Tabelle 35. Zusammenfassung von Interview 17 (I-17). 

Abkürzungsverzeichnis
A
Anforderung
$\mathrm{AD}$
Active Directory
A-F Funktionale Anforderung
A-R Randbedingung
A-Q Qualitätsanforderung
Abs. Absatz
I Interview
NDSG Niedersächsische Datenschutzgesetz
PM Probandenmanagementsystem
S Szenario
SAP IS-H SAP Industrial Solution for Healthcare
SUS System Usability Scale
TMF Technologie- und Methodenplattform der vernetzen medizinischen Forschung e. V.
UML Unified Modeling Language
vgl. vergleiche
Z Ziel
z. B. $\quad$ zum Beispiel 


\section{Anhang}

\section{A.1. Literaturrecherche}

Im Vordergrund der Literaturrecherche standen das Thema „Probandenmanagement“ sowie für die Methodik relevante Themenbereiche: Anforderungsanalyse, Konzeption und prototypische Implementierung sowie Validierung (Usability Test). Grundlage der Literaturrecherche bildeten die vorangegangen Veröffentlichungen im Rahmen dieser Dissertation [1, 7, 14, 109, 115118].

Insgesamt wurden vier Fachdatenbanken (PubMed ${ }^{9}$, IEEE Xplore Digital Library ${ }^{10}$, Google Scholar ${ }^{11}$ und Springer Link ${ }^{12}$ ) genutzt, in diesen wurden jeweils unterschiedliche deutsche und englische Suchbegriffe, für die verschiedenen Themenbereiche, verwendet. Die Suchergebnisse wurden nach dem Erscheinungsjahr absteigend sortiert; nachfolgend wurden zunächst die Überschriften untersucht und dann die Zusammenfassungen gelesen. Umfassten die Suchergebnisse zu viele Publikationen, wurde die Suchanfrage durch weitere Begriffe eingeschränkt oder eine Zeitspanne festgelegt.

\section{Probandenmanagement}

\begin{tabular}{|l|l|l|}
\hline proband management & patient recuirement & participant management \\
\hline participant tracking & research participant & clinical trial management \\
\hline protected health information & participant registry & improving recuirement \\
\hline Pseudonymisierung & pseudonymization & de-identification \\
\hline data protection & protected health information & clinical trial management \\
\hline protected health information & participant registry & improving recuirement \\
\hline secondary use & record for clinical research & data privacy \\
\hline record linkage & Probandenmanagement & \\
\hline
\end{tabular}

\section{Anforderungsanalyse}

\begin{tabular}{|l|l|l|}
\hline requirements & requirements engineering & requirements elicitation \\
\hline $\begin{array}{l}\text { goal based requirements en- } \\
\text { gineering }\end{array}$ & $\begin{array}{l}\text { scenario based requirements } \\
\text { engineering }\end{array}$ & goal scenario coupling \\
\hline
\end{tabular}

\section{Softwarearchitektur}

\begin{tabular}{|l|l|l|}
\hline software architecture & domain-driven design & clean architecture \\
\hline hexagonal architecture & ports and adapters & \\
\hline
\end{tabular}

\footnotetext{
${ }^{9}$ http://www.ncbi.nlm.nih.gov/pubmed

${ }^{10} \mathrm{http}: / /$ ieeexplore.ieee.org/Xplore/home.jsp

${ }^{11} \mathrm{http}: / /$ scholar.google.de

12 http://link.springer.com
} 


\begin{tabular}{|l|l|l|}
\hline prototyping & evolutionary prototyping & prototype approaches \\
\hline prototype benefits & & \\
\hline
\end{tabular}

Validierung (Usability Test)

\begin{tabular}{|l|l|l|}
\hline usability test & usability testing & usability evaluation \\
\hline usability benefits & participants usability testing & \\
\hline
\end{tabular}

\section{A.2. Interviewleitfaden}

\section{Hintergrund}

In (bio-) medizinischen Forschungsprojekten besteht großer Bedarf an einer effizienten und einheitlichen Verwaltung sowie der Nachverfolgung von Probanden über mehrere Forschungsprojekte. Im Rahmen dieser Dissertation wird der Bedarf am Beispiel der Universitätsmedizin Göttingen untersucht. Ziel der Dissertation ist die Konzeption und prototypische Implementierung eines Probandenmanagementsystems. Das System soll die Nachverfolgung von Probanden - über einen sehr langen Zeitraum und über mehrere Forschungsprojekte einer Forschungseinrichtung hinweg - effizient und einheitlich ermöglichen.

\section{Zielsetzung des Interviews}

Die Konzeption und prototypische Entwicklung des Probandenmanagementsystems erfordert die Erfassung von Szenarien im Rahmen des Probandenmanagements an der Universitätsmedizin Göttingen. Szenarien beschreiben die Interaktion von Benutzern oder externen Systemen mit dem zu entwickelnden Probandenmanagementsystem. Zur Gewinnung der Szenarien werden daher Interviews mit Mitarbeitern der Universitätsmedizin Göttingen durchgeführt, die ein besonderes Interesse am resultierenden Probandenmanagementsystem sowie einen direkten oder indirekten Einfluss auf die Gestaltung des Systems haben.

\section{Allgemeine Angaben}

1. Für welche Institution der Universitätsmedizin Göttingen arbeiten Sie?

2. Welche Rolle nehmen Sie in Ihrer Institution wahr?

3. Seit wann üben Sie diese Rolle aus?

4. An wie vielen Forschungsprojekten waren Sie bisher beteiligt?

5. An wie vielen Forschungsprojekten sind Sie aktuell beteiligt?

6. Sind Sie nur an lokalen oder auch an Forschungsprojekten mit weiteren Universitätskliniken beteiligt? Wenn ja, welche sind dies? 


\section{Umgang mit Probanden in Forschungsprojekten (Nur für Stakeholder mit direktem Kontakt zu Probanden)}

1. Wie hoch sind die Probandenzahlen in Ihren Forschungsprojekten im Durchschnitt?

2. Nutzen Sie technische Hilfsmittel zum Probandenmanagement (Stammdaten, Termine, Einwilligungen, Pseudonyme etc.) oder erfolgt diese rein papierbasiert?

3. Wenn ja, welche technischen Hilfsmittel (Excel, Access etc.) verwenden Sie?

4. Aus welchen Quellen rekrutieren Sie die Probanden? Werden die Probanden auch direkt aus der Station bzw. Ambulanz rekrutiert?

5. Rekrutieren Sie Probanden aus schon durchgeführten Forschungsprojekten?

6. Führen Sie eine Liste mit besonders geeigneten bzw. motivierten Probanden?

7. Wie kontaktieren Sie Ihre Probanden?

8. Nutzen Sie Pseudonyme zur Trennung der personenbezogenen Daten von den Forschungsdaten der Probanden?

9. Falls ja, wo speichern Sie die Zuordnung?

10. Sehen Sie in der Verwaltung Ihrer Probanden Optimierungspotential?

11. Falls ja, welche Verbesserungsvorschläge haben Sie?

\section{Umgang mit Probanden in Forschungsprojekten (Stakeholder ohne direktem Proban- denkontakt)}

1. Wissen Sie, wie aktuell Probanden in den Forschungsprojekten der Universitätsmedizin Göttingen verwaltet werden?

2. Wissen Sie, ob technische Hilfsmittel zum Management von Probanden innerhalb der Universitätsmedizin Göttingen zum Einsatz kommen?

3. Wenn ja, welche technischen Hilfsmittel (Excel, Access etc.) werden verwendet?

4. Stellen Sie selbst ein technisches Hilfsmittel zum Probandenmanagement zur Verfügung?

5. Falls ja, wäre es möglich, dass Sie mir Ihr technisches Hilfsmittel kurz vorstellen?

\section{Diskussion der vorgestellten Vorversion des Probandenmanagementsystems}

1. Entspricht das vorgestellte System Ihrer Vorstellung eines Probandenmanagementsystems?

2. Welche Ziele möchten Sie gerne mit einem solchen Probandenmanagementsystem erreichen?

3. Welche vorhandenen Abläufe könnten durch das Probandenmanagementsystem optimiert werden?

4. Welche Funktionen fehlen Ihnen für den Einsatz des Probandenmanagementsystems?

5. Welche Rahmenbedingungen sehen Sie für den Einsatz des Probandenmanagementsystems?

6. Könnten Sie sich vorstellen, dass Ärzte, Forscher und Studienassistenten oder Sie selbst Interesse an der Nutzung des Probandenmanagementsystems haben?

7. Wären Sie dazu bereit, an der Validierung des Probandenmanagementsystems teilzunehmen? 


\section{A.3. Usability-Test}

\section{Allgemeine Angaben}

Welche Rolle nehmen Sie an der Universitätsmedizin Göttingen ein?

Haben Sie direkten Kontakt zu Probanden bzw. Studienpatienten?

Wie werden Ihre Probanden aktuell verwaltet?

Nutzen Sie bereits das Probandenmanagementsystem des Instituts für Medizinische Informatik der Universitätsmedizin Göttingen? Falls ja, bitte beantworten Sie die folgenden Fragen:

F1: Ich nutze das System häufig.
stimme gar
(1)
(2)
(3)
(4)
(5) voll zu

F2: Ich finde das System unnötig komplex.
stimme gar
nicht zu
(1)
(2)
(3)
(4)
(5)
stimme
voll zu

F3: Ich finde das System einfach zu benutzen.
stimme gar
nicht zu
(1)
(2)
(3)
(4)
(5) voll zu

F4: Ich benötige die Hilfe einer technisch versierten Person, um das System benutzen zu können.
stimme gar
nicht zu
(1)
(2)
(3)
(4)
(5) stimme
voll zu

F5: Ich finde, die verschiedenen Funktionen in diesem System sind gut integriert.
stimme gar
nicht zu
(1)
(2)
(3)
(4)
(5)
stimme voll zu

F6: Ich denke, das System enthält zu viele Inkonsistenzen.
stimme gar
nicht zu
(1)
(2)
(3)
(4)
(5) voll zu 
F7: Ich kann mir vorstellen, dass die meisten Menschen den Umgang mit diesem System sehr schnell lernen.
stimme gar
nicht zu
(1)
(2)
(3)
(4)
(5)
stimme
voll zu

F8: Ich finde das System sehr umständlich zu nutzen.
stimme gar
nicht zu
(1)
(2)
(3)
(4)
(5) voll zu

F9: Ich fühle mich bei der Benutzung des Systems sehr sicher.
stimme gar
nicht zu
(1)
(2)
(3)
(4)
(5)
stimme
voll zu

F10: Ich musste eine Menge lernen, bevor ich anfangen konnte, das System zu verwenden.
stimme gar
nicht zu
(1)
(2)
(3)
(4)
(5) voll zu

\section{Szenario 1}

Das System bietet Ihnen über den Menüpunkt „Probandenübersicht“ eine Liste aller Probanden, die in mindestens einem Ihrer Forschungsprojekte eingeschlossen wurden. Suchen Sie nach „Peter Maier“, indem Sie in das Suchfeld „Maier“ eintragen. Entnehmen Sie der Übersicht, in welchen Forschungsprojekten er teilnimmt. Durch einen „Klick“ in die Tabelle erhalten Sie eine Übersicht seiner Stammdaten.

Ich finde die dargestellten Informationen sinnvoll und nützlich.
stimme gar
(1)
(2)
(3)
(4)
(5) voll zu

Bitte erläutern Sie kurz Ihre Antwort:

\section{Szenario 2}

„Peter Maier“ soll nun in das Forschungsprojekt „SFB42“ eingeschlossen werden. Wählen Sie hierzu das entsprechende Forschungsprojekt in der linken Menüleiste aus. Nach dem „Klick“ gelangen Sie zur Übersicht, in welcher Ihnen die erfassten Metadaten zum Projekt angezeigt werden.

Klicken Sie auf den Reiter „Forschungsakten“. Nachfolgend erhalten Sie eine Liste aller Probanden des Projekts und die Möglichkeit, eine neue Forschungsakte anzulegen. Klicken Sie auf „Neue Forschungsakte“. 
Im nachfolgenden Dialog können Sie auswählen, auf Basis welcher Probandendaten die neue Forschungsakte angelegt werden soll. Da „Peter Maier“ bereits in einem Ihrer Forschungsprojekte eingeschlossen wurde, wählen Sie die Option „Probanden auswählen“ aus. Nachfolgend erhalten Sie eine Liste all Ihrer Probanden.

Suchen Sie nach „Maier“ und wählen Sie „Peter Maier“ durch einen „Klick“ in der Tabelle aus. Klicken Sie auf „Weiter“ und füllen Sie die Angabe zur Forschungsakte nach Belieben aus. Durch einen „Klick“ auf „Speichern“ wird die neue Forschungsakte angelegt und angezeigt.

Ich finde die dargestellten Informationen sinnvoll und nützlich. stimme gar nicht zu
(2)
(3)
(4)
(5) voll zu

Bitte erläutern Sie kurz Ihre Antwort:

\section{Szenario 3}

Eine andere Möglichkeit eine Forschungsakte anzulegen besteht in der manuellen Eingabe der Probandenstammdaten. Wechseln Sie in das Projekt „PSYREG“ und legen Sie eine neue Forschungsakte mit folgenden Probandenstammdaten an: Vorname „Peter“, Nachname „Meier“, Geschlecht „männlich“ und Geburtsdatum „05.07.1980“.

Das System erkennt, dass der Proband mit den gleichen Stammdaten bereits im System angelegt wurde und verweist auf diesen. Wenn Sie zur Probandenübersicht wechseln und nach „Peter Maier“ suchen sehen Sie, dass dieser nun vier Forschungsprojekten zugeordnet ist.

Ich finde die dargestellten Informationen sinnvoll und nützlich.
stimme gar
(1)
(2)
(3)
(4)
(5) voll zu

Bitte erläutern Sie kurz Ihre Antwort:

\section{Szenario 4}

Durch die Integration des Systems mit dem Krankenhausinformationssystem ist der Abruf von Patientenstammdaten anhand der Patientenkennung möglich. Erstellen Sie eine neue Forschungsakte im Projekt „PSYREG“ mittels der Patientenkennung „AAABBB“. Am Schluss 
des Dialogs werden die Stammdaten des Patienten aus dem Krankenhausinformationssystem abgerufen.

In der nachfolgend dargestellten Übersicht der Forschungsakte von „Karl Huber“ bearbeiten Sie bitte seinen Status und hinterlegen Sie eine Anmerkung.

Ich finde die dargestellten Informationen sinnvoll und nützlich.
stimme gar
nicht zu
(1)
(2)
(3)
(4)
(5)
stimme
voll zu

Bitte erläutern Sie kurz Ihre Antwort:

Anmerkung: Änderungen der Stammdaten des Probanden sind nur durch einen Administrator möglich und das auch nur, wenn die Stammdaten nicht aus dem Krankenhausinformationssystem stammen. Ebenso können Forschungsakten nur durch einen Administrator aus dem Projekt gelöscht werden.

\section{Szenario 5}

Ein weiterer wesentlicher Bestandteil des Probandenmanagementsystems ist die Vernetzung mit externen Systemen. Dies wurde im Prototyp am Beispiel der Pseudonymisierung umgesetzt. Über den „Reiter“ Pseudonymisierung der Forschungsakte von „Karl Huber“ erhalten Sie eine Liste der Pseudonyme in diesem Forschungsprojekt. Legen Sie ein neues Pseudonym für „Karl Huber“ an und wählen Sie die Pseudonymisierung über ein externes System (externes Pseudonym) aus. Automatisch werden nachfolgend alle notwendigen Daten an das externe System übermittelt, welches anhand der Daten das Pseudonym generiert. Dieses wird abschließend lokal im Probandenmanagementsystem gespeichert.

Ich finde die dargestellten Informationen sinnvoll und nützlich.
stimme gar
nicht zu
(1)
(2)
(3)
(4)
5timme
voll zu

Bitte erläutern Sie kurz Ihre Antwort: 


\section{Systembewertung}

F1: Ich denke, dass ich das System gerne häufig benutzen würde.
stimme gar
nicht zu
(1)
(2)
(3)
(4)
(5)
stimme
voll zu

F2: Ich fand das System unnötig komplex.
stimme gar
nicht zu
(1)
(2)
(3)
(4)
(5) stimme
voll zu

F3: Ich fand das System einfach zu benutzen.
stimme gar
nicht zu
(1)
(2)
(3)
(4)
(5)
stimme
voll zu

F4: Ich glaube, ich würde die Hilfe einer technisch versierten Person benötigen, um das System benutzen zu können.
stimme gar
nicht zu
(1)
(2)
(3)
(4)
(5) stimme
voll zu

F5: Ich fand, die verschiedenen Funktionen in diesem System waren gut integriert.
stimme gar
nicht zu
(1)
(2)
(3)
(4)
(5)
stimme
voll zu

F6: Ich denke, das System enthielt zu viele Inkonsistenzen.
stimme gar
nicht zu
(1)
(2)
(3)
(4)
(5) stimme

F7: Ich kann mir vorstellen, dass die meisten Menschen den Umgang mit diesem System sehr schnell lernen.
stimme gar
nicht zu
(1)
(2)
(3)
(4)
(5) stimme

F8: Ich fand das System sehr umständlich zu nutzen.
stimme gar
nicht zu
(1)
(2)
(3)
(4)
(5)
stimme
voll zu

F9: Ich fühlte mich bei der Benutzung des Systems sehr sicher.
stimme gar
nicht zu
(1)
(2)
(3)
(4)
(5) stimme

F10: Ich musste eine Menge lernen, bevor ich anfangen konnte das System zu verwenden.
stimme gar
nicht zu
(1)
(2)
(3)
(4)
(5)
stimme 


\section{Systemfunktionen}

Um welche Funktionen sollte das System ergänzt werden? 


\section{A.4. Berechnung des Scores für den System Usability Scale}

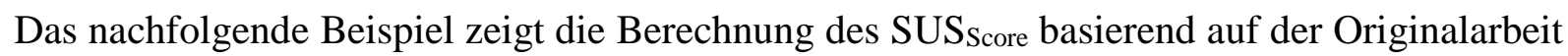
von Brooke [145]. Jede Antwort hat einen Wertebereich von 0 bis 4. Bei den Antworten 1, 3, 5, 7 und 9 wird dieser Wert um eins vermindert. Wohingegen bei den restlichen Antworten dieser Wert von der Zahl fünf subtrahiert wird. Alle Werte werden nachfolgend addiert und mit 2,5 multipliziert um den SUS Score $_{\text {zu erhalten. Im nachfolgenden Beispiel liegt der SUS }}$ Score bei 55.

F1: Ich denke, dass ich das System gerne häufig benutzen würde.
stimme gar
nicht zu
(1)
(2)
(3)
(4)
5) voll zu
4

F2: Ich fand das System unnötig komplex.
stimme gar
(1)
(2)
(3)
4
(5)
stimme
voll zu
1

F3: Ich fand das System einfach zu benutzen.
stimme gar
(1)
2 (3)
(4)
(5) voll zu
$\begin{array}{ll}\text { stimme } & 1 \\ \text { voll zu } & \end{array}$

F4: Ich glaube, ich würde die Hilfe einer technisch versierten Person benötigen, um das System benutzen zu können.
stimme gar
(1)
(3)
(4)
(5) voll zu
4

F5: Ich fand, die verschiedenen Funktionen in diesem System waren gut integriert.
stimme gar
(1)
2 (3)
(4)
(5)
stimme
voll zu
1

F6: Ich denke, das System enthielt zu viele Inkonsistenzen.
stimme gar
(1)
(2)
(4)
(5) voll zu

$\begin{array}{ll}\text { stimme } & 2 \\ \text { voll zu } & \end{array}$

F7: Ich kann mir vorstellen, dass die meisten Menschen den Umgang mit diesem System sehr schnell lernen.

stimme gar nicht zu

(1) 2

(4)

(5) voll zu 1

F8: Ich fand das System sehr umständlich zu nutzen.
stimme gar
nicht zu
(1)
(2)
(3)
4
(5) voll zu
1 
F9: Ich fühlte mich bei der Benutzung des Systems sehr sicher. stimme gar nicht zu
(1)
(2)
(3)
(4)
(5) stimme
4

F10: Ich musste eine Menge lernen, bevor ich anfangen konnte das System zu verwenden.

stimme gar nicht zu

(1) 2

(3)

(4)

(5) stimme

voll zu

3 


\section{A.5. Zusammenfassung der Interviews}

\begin{tabular}{|c|c|}
\hline Abschnitt & Inhalt \\
\hline Tätigkeitsbereich & Studienassistentin \\
\hline Forschungserfahrung & Ca. 70 klinische Studien \\
\hline Rekrutierung & $\begin{array}{l}\text { - Die Rekrutierung erfolgt auf der Station, in der Ambulanz } \\
\text { oder beim niedergelassenen Arzt. } \\
\text { - Probanden werden meist nicht aus vorhandenen Studien re- } \\
\text { krutiert, da dies bei interventionellen Studien verboten ist. }\end{array}$ \\
\hline Probandenverwaltung & $\begin{array}{l}\text { Papierbasiert mit handschriftlichen Ergänzungen auf Grund- } \\
\text { lage der Stammdaten aus SAP IS-H. }\end{array}$ \\
\hline Erwartung & $\begin{array}{l}\text { - Abbildung und Verbesserung Nachverfolgungsprozesses(Z-3, } \\
\text { Z-4, Z-5) } \\
\text { - Ablösung der papierbasierten Dokumentation(Z-4, Z-8, Z-9) } \\
\text { - Überblick über alle Probanden und Forschungsprojekte(Z-1, } \\
\text { Z-3, Z-5) } \\
\text { - Abruf von Stammdaten aus dem SAP IS-H anhand der Pati- } \\
\text { entenkennung(z-6) }\end{array}$ \\
\hline Sonstiges & $\begin{array}{l}\text { Die Suche von Probanden ist sehr aufwendig, da nur eine pa- } \\
\text { pierbasierte Dokumentation vorliegt. }\end{array}$ \\
\hline
\end{tabular}

Tabelle 19. Zusammenfassung von Interview 1 (I-1)

\begin{tabular}{l|l} 
Abschnitt & Inhalt \\
\hline Tätigkeitsbereich & Datenmanager für klinische Studien \\
\hline Forschungserfahrung & Ca. 20 klinische Studien \\
\hline Rekrutierung & Keine Angabe, da kein direkter Kontakt zu Probanden besteht. \\
\hline Probandenverwaltung & Keine Angabe, da kein direkter Kontakt zu Probanden besteht. \\
\hline Erwartung & $\begin{array}{l}\text { Überblick über alle Probanden und Forschungsprojekte (z-1, z-3, } \\
\text { Z-5) }\end{array}$ \\
\hline Sonstiges & $\begin{array}{l}- \text { Das Probandenmanagement könnte ergänzend zur bestehen- } \\
\text { den Software für Visitenplanung verwendet werden. }\end{array}$ \\
& - Verbesserung des Rekrutierungsprozesses für Probanden.
\end{tabular}

Tabelle 20. Zusammenfassung von Interview 2 (I-2) 


\begin{tabular}{l|l} 
Abschnitt & Inhalt \\
\hline Tätigkeitsbereich & Behördlicher Datenschutzbeauftragter \\
\hline Forschungserfahrung & $\begin{array}{l}\text { Beratung einer Vielzahl von medizinischen Forschungsprojek- } \\
\text { ten im Bereich des Datenschutzes. }\end{array}$ \\
\hline Rekrutierung & Keine Angabe, da kein direkter Kontakt zu Probanden besteht. \\
\hline Probandenverwaltung & Aus seiner Erfahrung basiert die Probandenverwaltung meist \\
& auf Excel-Tabellen. \\
\hline Erwartung & - Förderung der Transparenz durch die Darstellung, welche \\
& Daten von Probanden vorhanden und wo sie gespeichert \\
& sind (z-2) \\
& - Ersetzung individueller Lösungen zur Probandenverwaltung \\
& durch ein geregeltes Verfahren(Z-4, z-8, Z-9, z-10) \\
\hline Sonstiges & - Berücksichtigung von Empfehlungen und Regelungen des \\
& Datenschutzes. \\
& - Anbindung der Nutzerverwaltung der Universitätsmedizin \\
& Göttingen zur Sicherung der Anwendung. \\
& - Betrieb des Systems im gesicherten Intranet der Universi- \\
& tätsmedizin Göttingen.
\end{tabular}

Tabelle 21. Zusammenfassung von Interview 3 (I-3)

\begin{tabular}{l|l} 
Abschnitt & Inhalt \\
\hline Tätigkeitsbereich & Anwendungsbetreuerin für SAP IS-H \\
\hline Forschungserfahrung & Keine \\
\hline Rekrutierung & Keine Angabe, da kein direkter Kontakt zu Probanden besteht. \\
\hline Probandenverwaltung & Keine Angabe, da kein direkter Kontakt zu Probanden besteht. \\
\hline Erwartung & - Speicherung der wichtigsten personenbezogenen Doku- \\
& mente, beispielsweise der Einwilligungserklärung(Z-12) \\
& - Abruf von Stammdaten aus dem SAP IS-H anhand der Pati- \\
& entenkennung(Z-6) \\
& - Prüfung, ob der Patient ein Proband in einem Forschungs- \\
& projekt ist(Z-6) \\
\hline Sonstiges & Rudimentäre Erweiterung in SAP IS-H, welche die Markie- \\
& rung von Patienten als Studienteilnehmer ermöglicht.
\end{tabular}

Tabelle 22. Zusammenfassung von Interview 4 (I-4) 


\begin{tabular}{l|l} 
Abschnitt & Inhalt \\
\hline Tätigkeitsbereich & Wissenschaftliche Mitarbeiterin (promotivierte Psychologin) \\
\hline Forschungserfahrung & Ca. 8 Forschungsprojekte (Lokal und Verbund) \\
\hline Rekrutierung & $\begin{array}{l}\text { Rekrutierung erfolgt aus früheren Forschungsprojekten, von } \\
\text { der Station oder Kontrollen durch Listen des Einwohnermel- } \\
\text { deamts. }\end{array}$ \\
\hline Probandenverwaltung & - Excel-Tabelle mit papierbasierten Ergänzungen \\
& - Einsatz der Vorversion des Probandenmanagementsystems \\
\hline Erwartung & - Unterstützung bei der Rekrutierung von Probanden aus be- \\
& reits durchgeführten Forschungsprojekten(Z-7) \\
& - Abruf von Stammdaten der Probanden aus SAP IS-H (Z-6) \\
& - Übersicht über alle Probanden und Forschungsprojekte, an \\
& denen der Forscher beteiligt ist(Z-1, Z-3, Z-5) \\
& - Abruf und Speicherung von Pseudonymen aus externen \\
& Systemen zur zentralen Pseudonymisierung(Z-14, Z-15) \\
\hline Sonstiges & Keine Angaben
\end{tabular}

Tabelle 23. Zusammenfassung von Interview 5 (I-5)

\begin{tabular}{l|l} 
Abschnitt & Inhalt \\
\hline Tätigkeitsbereich & IT-Koordinator \\
\hline Forschungserfahrung & $\begin{array}{l}\text { Ansprechpartner bei IT-Fragen für Ärzte, Forscher, Wissen- } \\
\text { schaftler und Studienpersonal. }\end{array}$ \\
\hline Rekrutierung & Keine Angabe, da kein direkter Kontakt zu Probanden besteht. \\
\hline Probandenverwaltung & - Excel-Tabellen \\
& - Papierbasierte Dokumentation \\
& - Outlook-Kalender zur Visitenplanung \\
\hline Erwartung & - Projektübergreifendes Management von Probanden zur Re- \\
& duzierung des Arbeitsaufwandes(Z-1, Z-3, Z-4, Z-5, Z-8, Z-9) \\
& - Abruf von Stammdaten der Probanden aus SAP IS-H (Z-6) \\
\hline Sonstiges & - Verbesserung des Rekrutierungsprozesses für Probanden \\
& durch die Erfassung von Metadaten. \\
& - Verwendung der zentralen Benutzerverwaltung der Univer- \\
& sitätsmedizin Göttingen. \\
& - Das System sollte eine Webanwendung sein, die mit allen \\
& gängigen Webbrowsern ohne zusätzliche Software (Java, \\
& Flash etc.) genutzt werden kann.
\end{tabular}

Tabelle 24. Zusammenfassung von Interview 6 (I-6) 


\begin{tabular}{l|l} 
Abschnitt & Inhalt \\
\hline Tätigkeitsbereich & Wissenschaftliche Mitarbeitern (promovierte Physiotherapeu- \\
& tin)
\end{tabular}

\begin{tabular}{l|l} 
Abschnitt & Inhalt \\
\hline Tätigkeitsbereich & Studienassistentin (Medizinische Dokumentarin) \\
\hline Forschungserfahrung & Ca. 4 Verbundforschungsprojekte \\
\hline Rekrutierung & - Von der Station durch den behandelnden Arzt \\
& - Nach Zustimmung der Ethikkommission durch Kontaktauf- \\
& nahme zu früheren Patienten \\
& - Aushänge in Klinik \\
& - Probanden aus vorhandenen Forschungsprojekten \\
\hline Probandenverwaltung & - Excel-Tabelle mit papierbasierten Ergänzungen \\
& - Einsatz der Vorversion des Probandenmanagementsystems \\
\hline Erwartung & - Verbesserung der Transparenz durch eine Übersichtliche \\
& Darstellung der Probanden(Z-1, Z-2, Z-3, Z-4, Z-5) \\
& - Unterstützung von statistischen Auswertungen entweder di- \\
& rekt durch das System oder durch geeignete Exporte(Z-11) \\
& - Abruf der Pseudonyme von der zentralen Anwendung zur \\
& Pseudonymisierung der Probanden(Z-14, Z-15) \\
\hline Sonstiges & Vermeidung von Fehleingaben und Sicherung der Datenbank.
\end{tabular}




\begin{tabular}{l|l} 
Abschnitt & Inhalt \\
\hline Tätigkeitsbereich & $\begin{array}{l}\text { Leitender Mitarbeiter im Geschäftsbereich Informationstech- } \\
\text { nologie }\end{array}$ \\
\hline Forschungserfahrung & Ca. 10 Forschungsprojekte \\
\hline Rekrutierung & Keine Angabe, da kein direkter Kontakt zu Probanden besteht. \\
\hline Probandenverwaltung & Excel-Tabellen \\
\hline Erwartung & - Ablösung der Excel-Tabellen durch ein Softwaresystem(Z-4, \\
& z-10) \\
& Dokumentation der Probandenstammdaten sowie der Ein- \\
& willigungserklärung(Z-4, Z-8, Z-9, z-12) \\
& - Pseudonymisierung der Probanden und gesicherte Rückauf- \\
& lösung der Pseudonyme(Z-13, Z-14, Z-16) \\
& - Überblick über alle Forschungsprojekte und den an ihnen \\
& beteiligten Probanden(Z-1, Z-3, Z-5) \\
\hline Sonstiges & - Ein Probandenmanagementsystem wird in jedem For- \\
& schungsprojekt gebraucht. \\
& - Die Nutzerverwaltung sollte die bestehende Infrastruktur \\
& der Universitätsmedizin Göttingen verwenden. \\
& - Berücksichtig der Good Clinical Practice (GCP) und der \\
& Good Automated Manufacturing Practice (GAMP).
\end{tabular}

Tabelle 27. Zusammenfassung von Interview 9 (I-9)

\begin{tabular}{|c|c|}
\hline Abschnitt & Inhalt \\
\hline Tätigkeitsbereich & Facharzt für Neuroradiologie \\
\hline Forschungserfahrung & Ca. 70 Forschungsprojekte \\
\hline Rekrutierung & $\begin{array}{l}\text { - Tagesklinik, Poliklinik, Forschungsnetzn oder aus bereits } \\
\text { durchgeführten Forschungsprojekten } \\
\text { - Erfolgt meist gemeinsam mit unterschiedlichen Fachberei- } \\
\text { chen } \\
\text { - Excel-Tabelle mit Probanden, die besonders interessiert an } \\
\text { Forschungsprojekten sind }\end{array}$ \\
\hline Probandenverwaltung & Excel-Tabellen \\
\hline Erwartung & $\begin{array}{l}\text { - Überblick über alle Probanden und Forschungsprojekte so- } \\
\text { wie Angaben über den Rekrutierungsstand }(\mathrm{Z}-1, \mathrm{Z}-3 \text {, Z-5) } \\
\text { - Unterstützung bei der Verknüpfung von Probanden und de- } \\
\text { ren Pseudonymen(Z-13, Z-14, Z-16) } \\
\text { - Vereinheitlichung der Excel-Tabellen durch eine Soft- } \\
\text { } \text { ware }(\mathrm{Z}-4, \mathrm{Z}-10)^{\text {- }}\end{array}$ \\
\hline Sonstiges & $\begin{array}{l}\text { Vereinfachung der Kommunikation mit den anderen Fachbe- } \\
\text { reichen durch ein gemeinsames Informationssystem. }\end{array}$ \\
\hline
\end{tabular}

Tabelle 28. Zusammenfassung von Interview 10 (I-10) 


\begin{tabular}{|c|c|}
\hline Abschnitt & Inhalt \\
\hline Tätigkeitsbereich & $\begin{array}{l}\text { Leitende wissenschaftliche Mitarbeiterin (Institut für Medizi- } \\
\text { nischen Informatik) }\end{array}$ \\
\hline Forschungserfahrung & $\begin{array}{l}\text { Beratung von ca. } 10 \text { Forschungsprojekten in Fragen zur IT- } \\
\text { Infrastruktur von Biobanken }\end{array}$ \\
\hline Rekrutierung & Keine Angabe, da kein direkter Kontakt zu Probanden besteht. \\
\hline Probandenverwaltung & Keine Angabe, da kein direkter Kontakt zu Probanden besteht. \\
\hline Erwartung & $\begin{array}{l}\text { - Meldung von Probanden an zentrale Erfassungssysteme von } \\
\text { Forschungsprojekten zur Pseudonymisierung(Z-15) } \\
\text { - } \text { Abbildung der informierten Einwilligungserklärung und de- } \\
\text { ren Widerruf(Z-12) } \\
\text { - Datenzusammenführung, durch Bereitstellung von zusam- } \\
\text { mengehörigen Pseudonymen für einen Probanden(Z-16) } \\
\text { - Möglichkeit statistischen Auswertungen über mehrere For- } \\
\text { schungsprojekte durchzuführen (Z-11, Z-16) }\end{array}$ \\
\hline Sonstiges & $\begin{array}{l}\text { Umsetzung von Empfehlungen und Regularien des Daten- } \\
\text { schutzes, sodass ein Zugriff auch von externen Benutzern } \\
\text { möglich ist. }\end{array}$ \\
\hline
\end{tabular}

Tabelle 29. Zusammenfassung von Interview 11 (I-11)

\begin{tabular}{l|l} 
Abschnitt & Inhalt \\
\hline Tätigkeitsbereich & Wissenschaftlicher Leiter des Bereichs für psychiatrische Ge- \\
& netik
\end{tabular}

Tabelle 30. Zusammenfassung von Interview 12 (I-12) 


\begin{tabular}{l|l} 
Abschnitt & Inhalt \\
\hline Tätigkeitsbereich & Oberärztin in der Neuropathologie \\
\hline Forschungserfahrung & Mehrere Forschungsprojekte \\
\hline Rekrutierung & $\begin{array}{l}\text { Rekrutierung erfolgt aus der Routine, insbesondere durch den } \\
\text { konsiliarischen Dienst in Kooperation mit den behandelnden } \\
\text { Ärzten. }\end{array}$ \\
\hline Probandenverwaltung & $\begin{array}{l}\text { Access Datenbank, Excel-Tabellen und schriftliche Dokumen- } \\
\text { tation, die als Sicherung verwendet wird. }\end{array}$ \\
\hline Erwartung & $\begin{array}{l}\text { - Langfristige Nachverfolgung von Probanden(Z-3, Z-4, Z-5) } \\
\text { - Dokumentation der Probandenstammdaten(Z-4, Z-8, Z-9) }\end{array}$ \\
\hline Sonstiges & Verbesserung der Kommunikation mit den Probanden
\end{tabular}

Tabelle 31. Zusammenfassung von Interview 13 (I-13)

\begin{tabular}{l|l} 
Abschnitt & Inhalt \\
\hline Tätigkeitsbereich & Facharzt für Neuropathologie \\
\hline Forschungserfahrung & Leiter der deutschen Brainbank für Multiple Sklerose und \\
& mehrere kleinere Forschungsprojekte \\
\hline Rekrutierung & - Rekrutiert gemeinsam mit anderen Fachbereichen \\
& - Rekrutierung von Probanden aus auch vorhandenen For- \\
& schungsprojekten \\
\hline Probandenverwaltung & - Excel-Tabellen \\
\hline Erwartung & - Ablösung der vorhandenen Excel-Tabellen (Z-4, Z-10) \\
& - Nutzung des Probandenmanagements für mehrere For- \\
& schungsprojekte(Z-1, Z-4, Z-5) \\
& - Nachverfolgung von Probanden über einen langen \\
& Zeitraum(Z-3, Z-4, Z-5) \\
& - Bereitstellung eines Systems, das die anderen eingesetzten \\
& Forschungssysteme zusammenhält(Z-2) \\
\hline Sonstiges & Keine Angabe
\end{tabular}

Tabelle 32. Zusammenfassung von Interview 14 (I-14)

\begin{tabular}{|c|c|}
\hline Abschnitt & Inhalt \\
\hline Tätigkeitsbereich & Assistenzarzt für Psychiatrie und Psychotherapie \\
\hline Forschungserfahrung & Ca. 7 Forschungsprojekte \\
\hline Rekrutierung & $\begin{array}{l}\text { Ca. } 20 \% \text { der Patienten werden für Forschungsprojekte rekru- } \\
\text { tiert. }\end{array}$ \\
\hline Probandenverwaltung & Excel-Tabellen \\
\hline Erwartung & $\begin{array}{l}\text { - Ablösung der diversen Excel-Tabellen durch eine einheitli- } \\
\text { che Softwareanwendung }(\mathrm{Z}-4, \mathrm{Z}-10) \\
\text { - Überblick über die aktuellen Probanden und deren For- } \\
\text { schungsprojekte }(\mathrm{Z}-1, \mathrm{Z}-3, \mathrm{Z}-5)\end{array}$ \\
\hline Sonstiges & Keine Angabe \\
\hline
\end{tabular}




\begin{tabular}{l|l} 
Abschnitt & Inhalt \\
\hline Tätigkeitsbereich & Oberarzt für ambulante Onkologie \\
\hline Forschungserfahrung & Ca. 30 klinische Studien \\
\hline Rekrutierung & $\begin{array}{l}\text { - Probanden werden aus dem Tumorboard und der onkologi- } \\
\text { schen Ambulanz rekrutiert } \\
\text { - Die Rekrutierung der Probanden erfolgt meist in Koopera- } \\
\\
\text { tion mit der Pathologie }\end{array}$ \\
\hline Probandenverwaltung & Schriftliche Studienakte / Patientenakte \\
\hline Erwartung & - Nachverfolgung von Probanden, sodass das Auftreten eines \\
& Rezidivs dokumentiert werden kann(Z-3, Z-4, Z-5) \\
& - Bereitstellung und Implementierung von Schnittstellen zu \\
& bestehenden Informationssysteme(Z-6, Z-15) \\
& - Nutzung von vorhandenen Datenquellen, sodass keine er- \\
& neute Eingabe der Daten notwendig ist(Z-6) \\
\hline Sonstiges & Keine Angaben
\end{tabular}

Tabelle 34. Zusammenfassung von Interview 16 (I-16)

\begin{tabular}{|c|c|}
\hline Abschnitt & Inhalt \\
\hline Tätigkeitsbereich & $\begin{array}{l}\text { Studienassistentin in der Klinik für Psychiatrie und Psycho- } \\
\text { therapie }\end{array}$ \\
\hline Forschungserfahrung & Ca. 10 klinische Studien \\
\hline Rekrutierung & $\begin{array}{l}\text { - Rekrutierung von Probanden für multizentrische Studien } \\
\text { - Probanden werden aus der Ambulanz rekrutiert } \\
\text { - Rekrutierung aus bereits durchgeführten Forschungsprojek- } \\
\text { ten }\end{array}$ \\
\hline Probandenverwaltung & Excel-Tabellen mit schriftliche Ergänzungen \\
\hline Erwartung & $\begin{array}{l}\text { - Ersetzung der verschiedenen Excel-Tabellen durch eine ein- } \\
\text { heitliche Softwarelösung zur Probandenverwaltung für alle } \\
\text { Forschungsprojekte(Z-1, Z-3, Z-4, Z-5, U-8, Z-9, Z-10) } \\
\text { - Abruf von zentralen Pseudonymen durch eine zentrale Soft- } \\
\text { wareanwendung oder telefonisch(Z-15) } \\
\text { - Zuordnung der Pseudonyme zu den personenbezogenen Da- } \\
\text { ten der Probanden(Z-14) }\end{array}$ \\
\hline Sonstiges & Keine Angabe \\
\hline
\end{tabular}




\section{A.6. Ergänzungen zum Architekturkonzept}

\section{Domänenmodell}

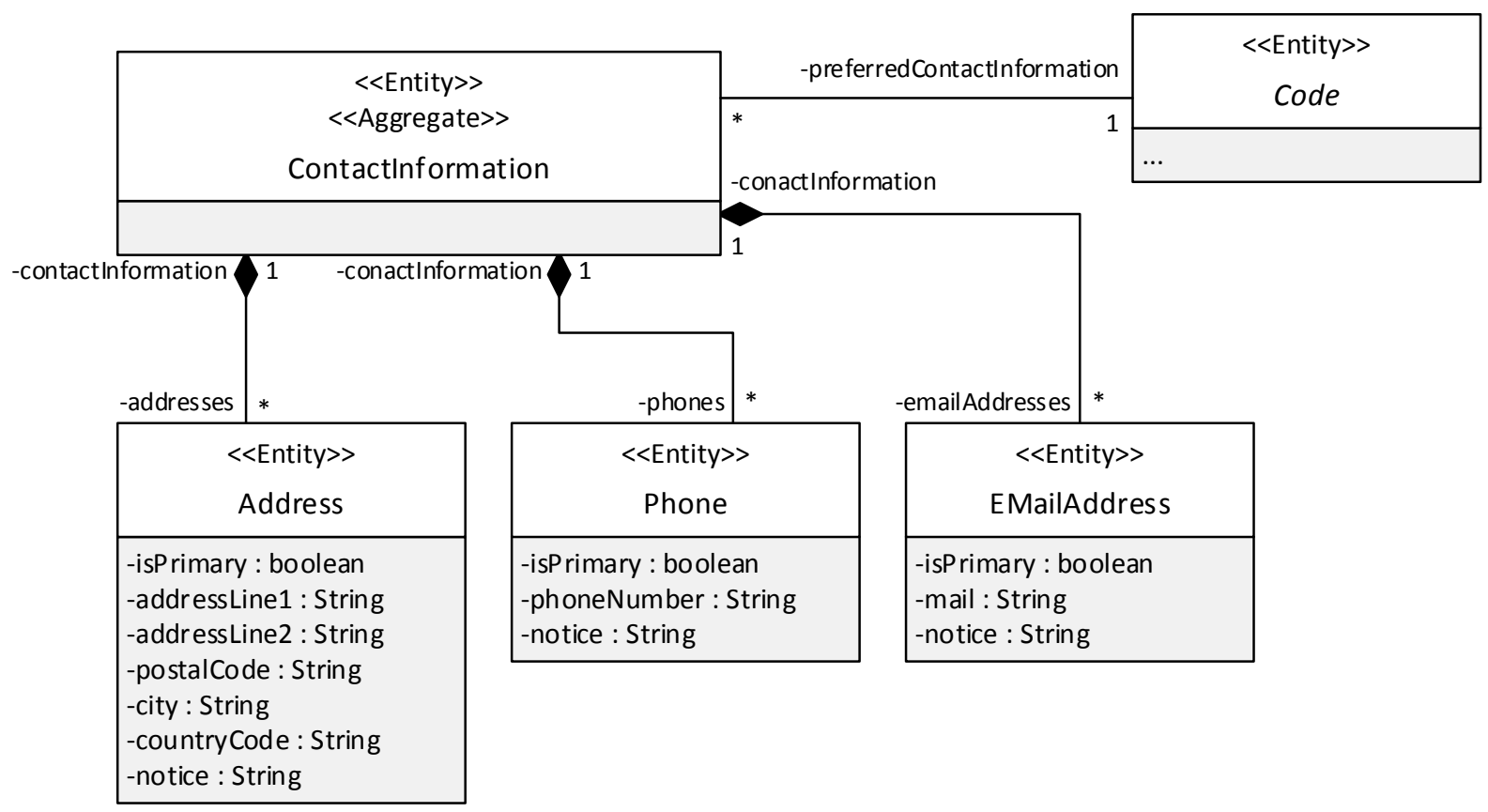

Abbildung 69. Ergänzung des Domänenmodells des Bereichs Probandenverwaltung um Kontaktinformationen zu einem Probanden

\section{Primäre und sekundäre Schnittstellen}

\begin{tabular}{|l|l|}
\hline \multicolumn{1}{|c|}{ CodeService } \\
\hline $\begin{array}{l}\text { +save(code : Code) : Code } \\
\text { +delete(code : Code) } \\
\text { +findSystemCodes(scope : Scope) }: \text { List }<\text { SystemCode }> \\
\text { +findProjectCodes(scope : Scope, project : Project) }: \text { List<ProjectCode }>\end{array}$
\end{tabular}

\begin{tabular}{|l|l|}
\hline \multicolumn{1}{|c|}{ ReportService } \\
\hline $\begin{array}{l}\text { +save(report : Report) : Report } \\
\text { +delete(report : Report) } \\
\text { +findAll() : List<Report> } \\
\text { +findBy(userAccount : UserAccount) : List<Report> } \\
\text { +findBy(id : Long, userAccount : UserAccount) : Report }\end{array}$
\end{tabular}

Abbildung 70. Primäre und sekundäre Schnittstellen zur Verwaltung der Entities des Bereichs Systemkonfiguration 


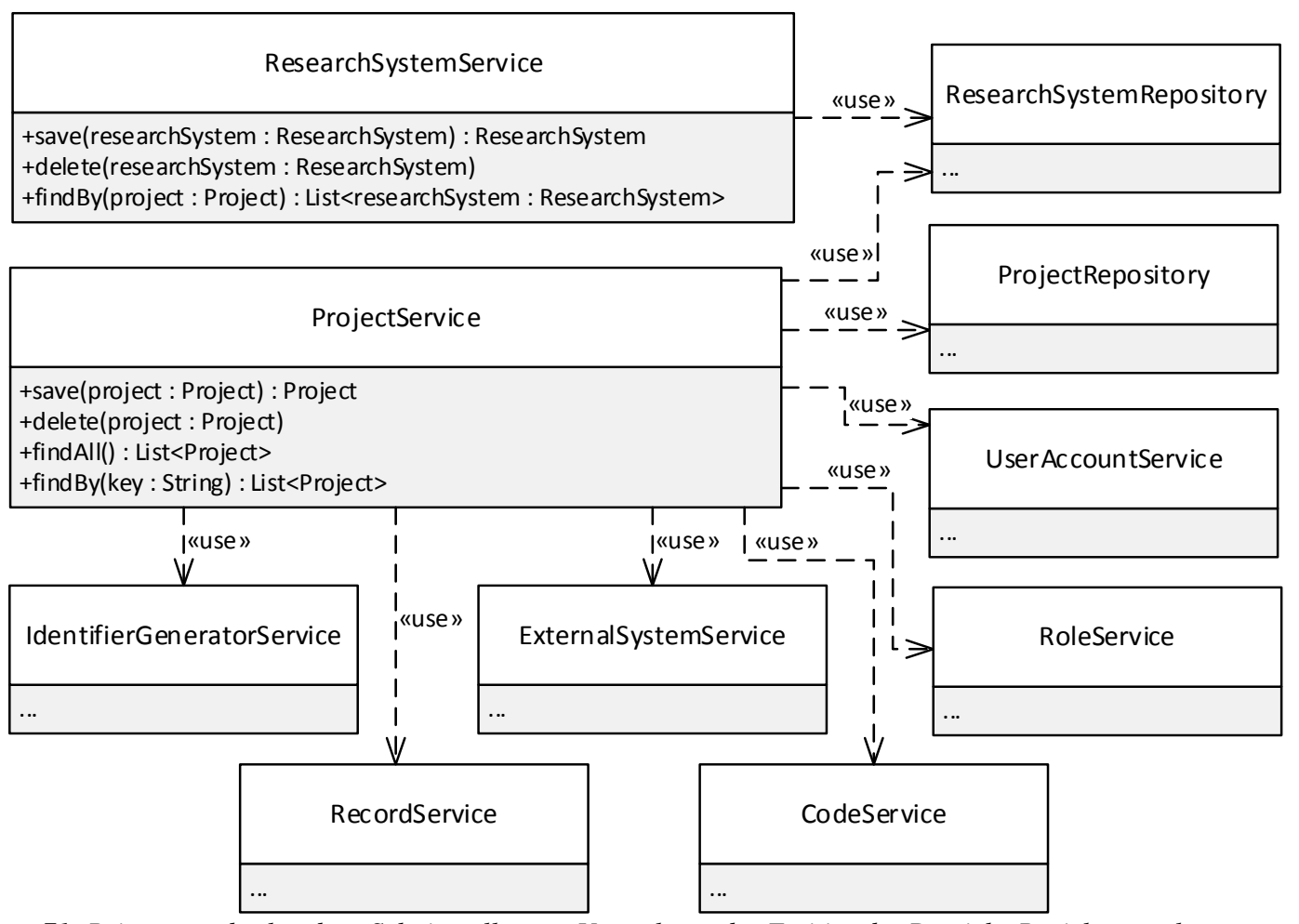

Abbildung 71. Primäre und sekundäre Schnittstellen zur Verwaltung der Entities des Bereichs Projektverwaltung

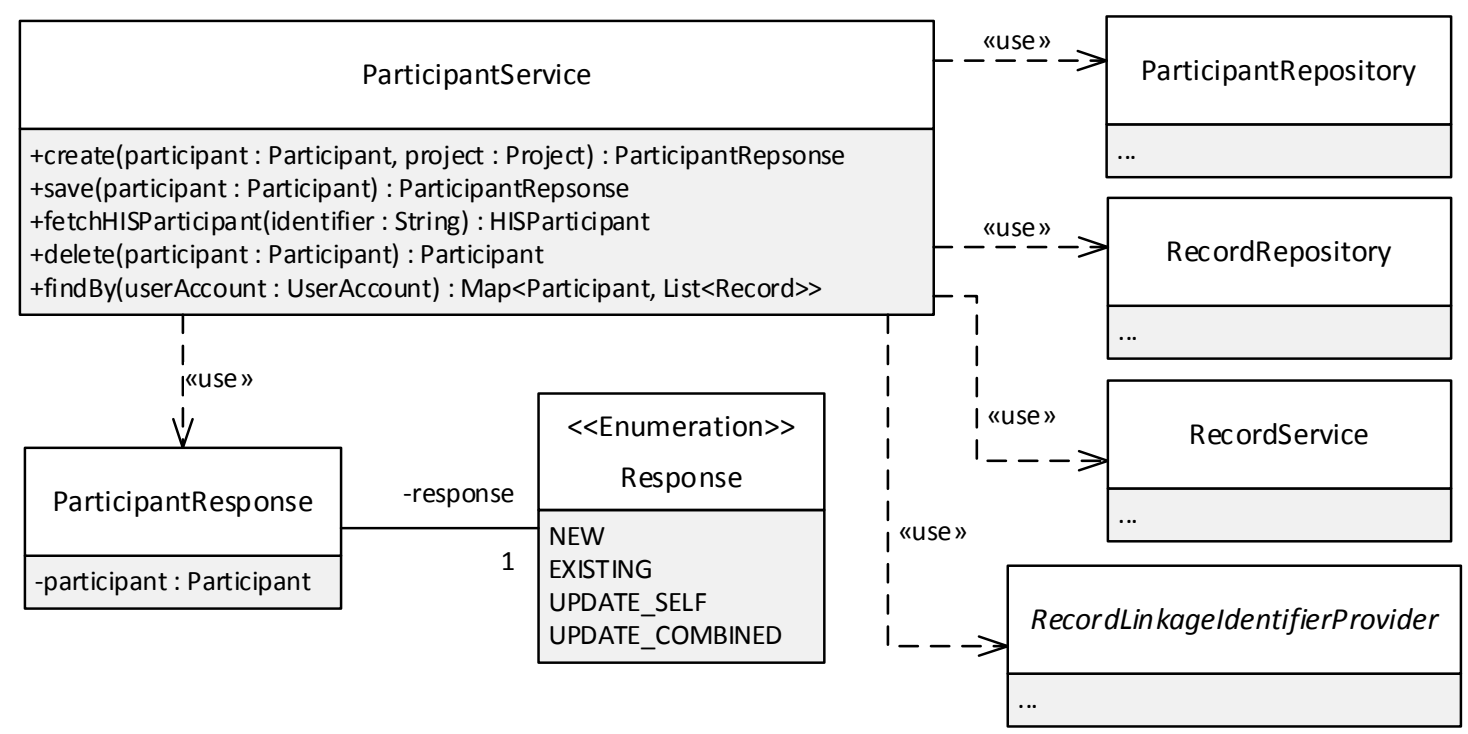

Abbildung 72. Primäre und sekundäre Schnittstellen zur Verwaltung der Entities des Bereichs Probandenverwaltung 


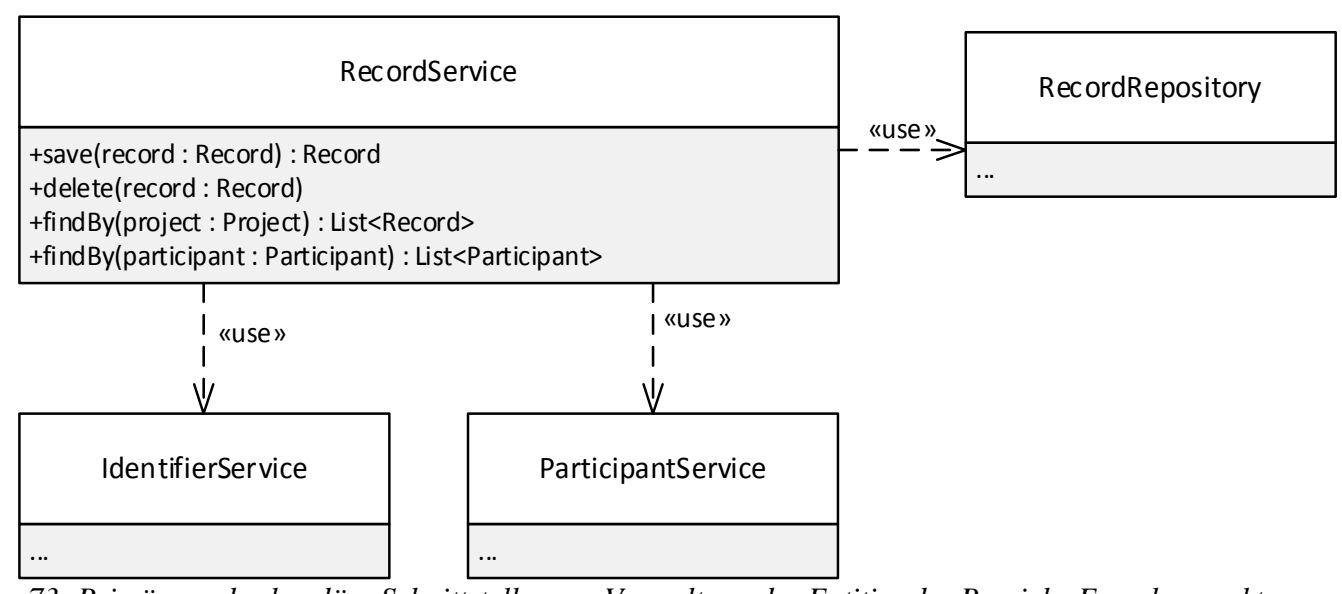

Abbildung 73. Primäre und sekundäre Schnittstellen zur Verwaltung der Entities des Bereichs Forschungsakte

\begin{tabular}{|l|l|}
\hline \multicolumn{1}{|c|}{ ExternalSystemService } \\
\hline $\begin{array}{l}\text { +save(externalSystem : ExternalSystem) : ExternalSystem } \\
\text { +delete(externalSystem: ExternalSystem) } \\
\text { +findBy(project : Project) : List<ExternalSystem> }\end{array}$
\end{tabular}

Abbildung 74. Primäre und sekundäre Schnittstellen zur Verwaltung der Entities des Bereichs Kommunikation
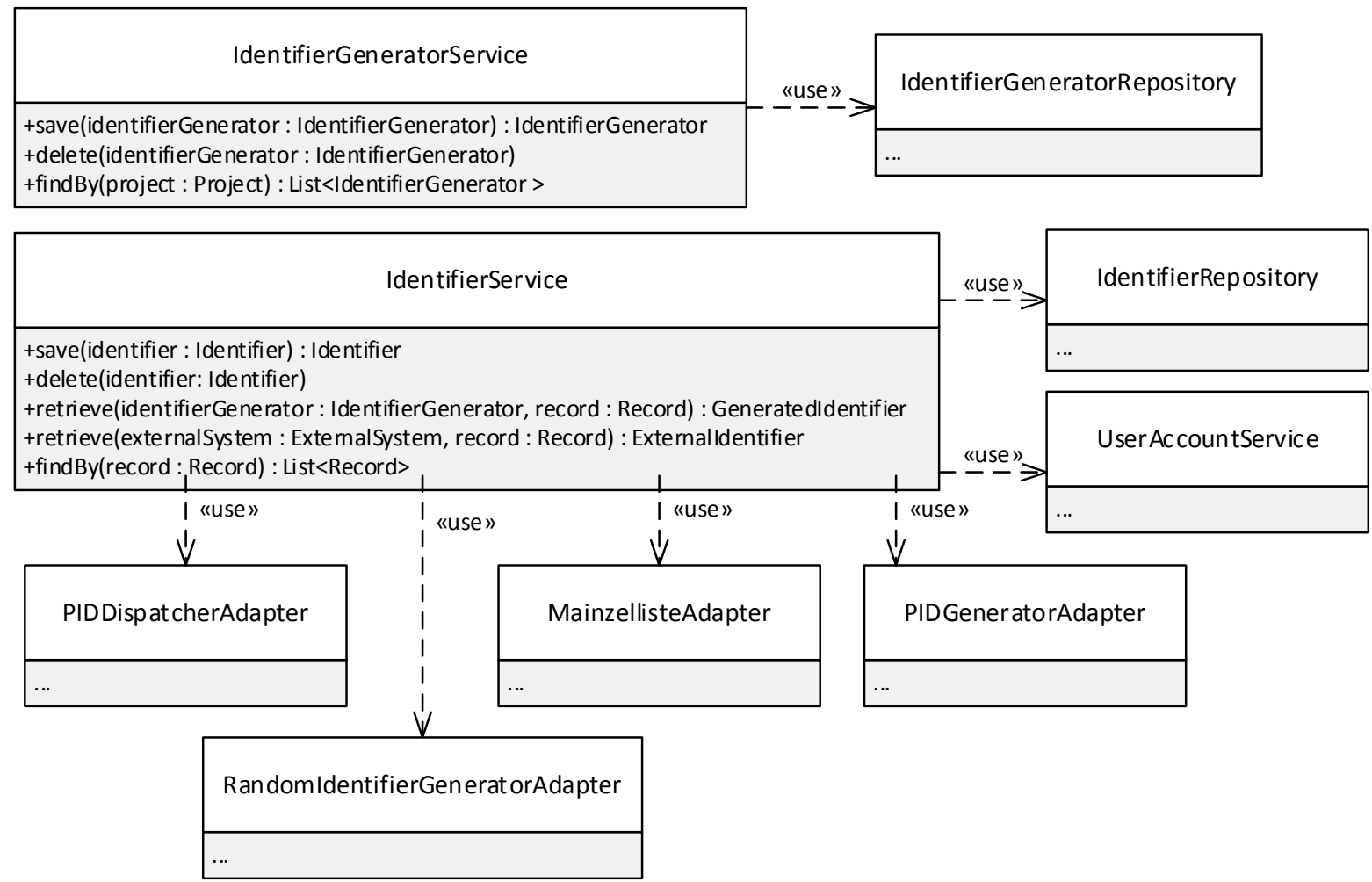

Abbildung 75. Primäre und sekundäre Schnittstellen zur Verwaltung der Entities des Bereichs Pseudonymisierung 
A.7. DVD-Beigabe

Die DVD-Beigabe umfasst

- den Quellcode des Probandenmanagementsystems,

- die Usability-Tests (pseudonymisiert) sowie

- eine PDF-Datei dieser Dissertation. 


\section{Lebenslauf}

\section{Persönliche Daten}

$\begin{aligned} \text { Name } & \text { Jens Schwanke } \\ \text { Geburtsdatum- und ort } & \text { 14.02.1986, Heidelberg } \\ \text { Familienstand } & \text { verheiratet } \\ \text { Staatsangehörigkeit } & \text { deutsch }\end{aligned}$

\section{Berufserfahrung}

seit 07/2015 Projektleiter, Kairos GmbH, Bochum.

09/2011 - 06/2015 Wissenschaftlicher Mitarbeiter, Institut für Medizinische Informatik, Universitätsmedizin Göttingen.

03/2009 - 02/2010 Studentische Hilfskraft, Hochschule Heilbronn.

11/2007 - 02/2009 Studentische Hilfskraft, Reinshagen \& Hartung GmbH.

\section{Wissenschaftliche Ausbildung}

2015 Session Chair, 60. Jahrestagung der Deutsche Gesellschaft für Medizinische Informatik, Biometrie und Epidemiologie e.V., Krefeld

2013 Reviewer, IEEE Transactions on Haptics

seit 01/2012 Doktorand, Georg-August University School of Science, Georg-August-Universität Göttingen.

Konzeption und Entwicklung eines Probandenmanagementsystems am Beispiel der Universitätsmedizin Göttingen

01/2011 - 07/2011 Stipendiat, Hasso-Plattner-Institut, Universität Potsdam.

03/2006 - 02/2011 Diplomstudium der Medizinischen Informatik, ReuprechtKarls-Universität Heidelberg / Hochschule Heilbronn Design und Implementierung eines Atem-Coaching-Systems für die adaptive, atemgetriggerte Strahlentherapie 


\section{Veröffentlichungen (TOP 5)}

Stahmann A, Bauer CRKD, Schulze TG und Schwanke J. Standortbezogene Datenzusammenführung in Verbundforschungsvorhaben durch ein Probandenmanagementsystem. 59. Jahrestagung der Deutschen Gesellschaft für Medizinische Informatik, Biometrie und Epidemiologie 2014

Stahmann A, Bauer CRKD und Schwanke J. Concept for Linking De-identified Biomedical Research Data Using a Study Participant Management System. Study Health Technology Informatics. 2014:205(1);848-52

Schwanke J, Nussbeck SY, Helbing K und Rienhoff O. Entwicklung eines Probandenmanagementsystems für ein multizentrisches Forschungsprojekt. 58. Jahrestagung der Deutschen Gesellschaft für Medizinische Informatik, Biometrie und Epidemiologie 2013

Schwanke J, Rienhoff O, Schulze TG und Nussbeck SY. Suitability of Customer Relationship Management Systems for the Management of Study Participants in Biomedical Research. Methods of Information Medicine. 2013;52(4):340-50

Demiroglu SY, Skrowny D, Quade M, Schwanke J, Budde M, Gullatz V, Reich-Erkelenz D, Jakob JJ, Falkai P, Rienhoff O, Helbing K, Heilbronner U und Schulze TG. Managing Sensitive Phenotypic Data and Biomaterial in Large-Scale Collaborative Psychiatric Genetic Research Projects: Practical Considerations. Molecular Psychiatry. 2012; 17(12):1180-1185 